\title{
Haptic Physical Human Assistance
}

\section{Arvid Keemink}
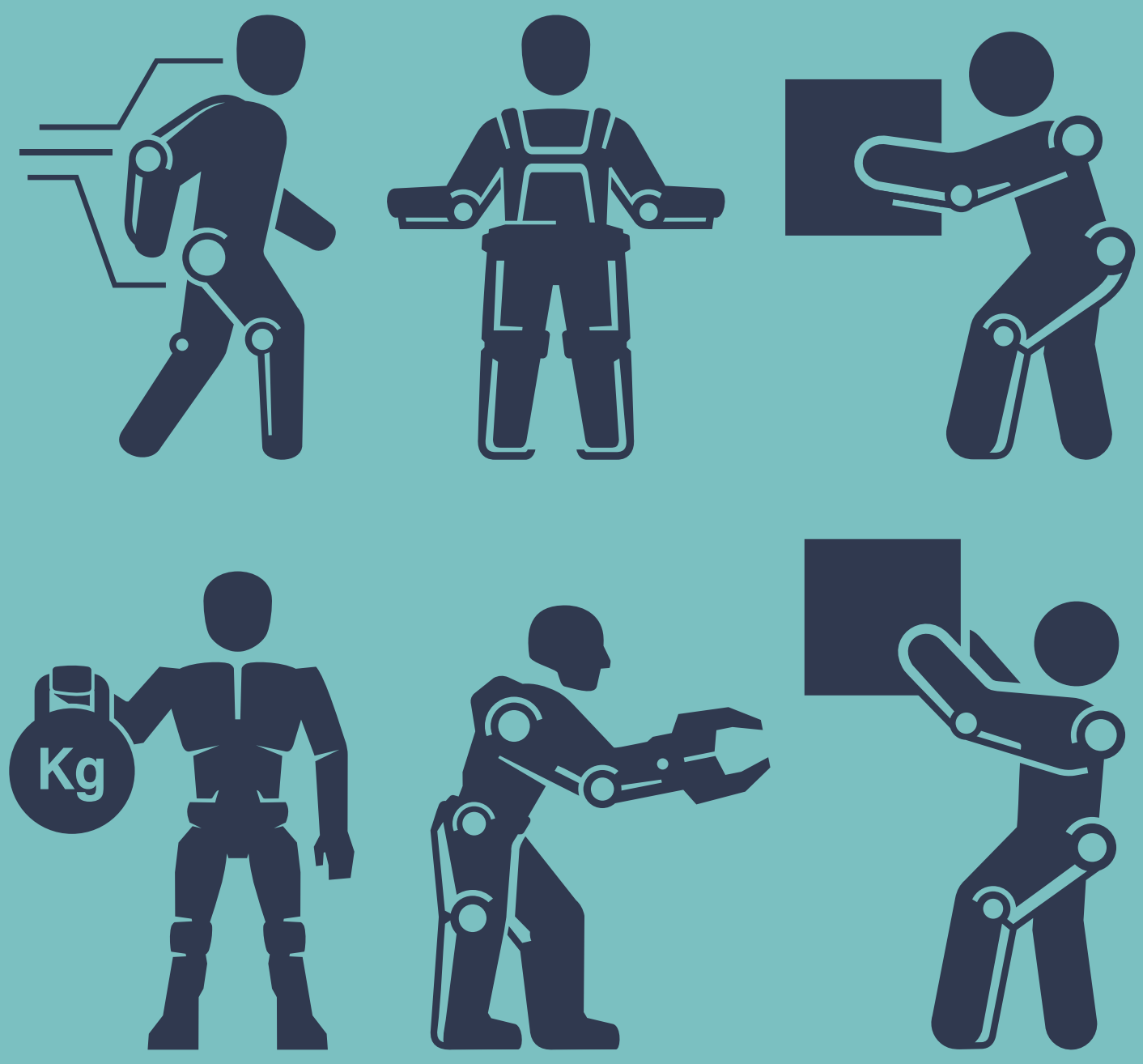

HAPTIC PHYSICAL HUMAN ASSISTANCE 
Samenstelling promotiecommissie:

VOORZITTER EN SECRETARIS

Prof. dr. G.P.M.R. Dewulf

Universiteit Twente

PROMOTOR

Prof. dr. ir. H. van der Kooij Universiteit Twente

CO-PROMOTOR

Dr. ir. A.H.A. Stienen

Universiteit Twente

LEDEN

Prof. dr. ir. S. Stramigioli Universiteit Twente

Prof. dr. A.A. Stoorvogel Universiteit Twente

Prof. dr. ir. A. de Boer Universiteit Twente

Prof. dr. E. Burdet Imperial College (London, UK)

Dr. ir. D.A. Abbink Technische Universiteit Delft

\author{
PARANIMFEN \\ Ir. K. Nizamis \\ Ir. N.W.M. Beckers
}

Printed by Gildeprint, www.gildeprint.nl

Cover image: VoodooDot/Shutterstock

ISBN: 978-90-365-4408-5

DOI: $10.3990 / 1.9789036544085$

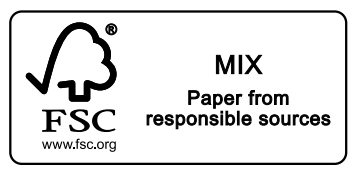

Copyright $@ 2017$ Arvid Quintijn Léon Keemink, Zutphen, The Netherlands. This dissertation is published under the terms of the Creative Commons Attribution NonCommercial 4.0 International License, which permits unrestricted use, distribution, and reproduction in any medium, provided the original work is properly credited. The Creative Commons Public Domain Dedication waiver applies to the data made available in this publication, unless otherwise stated. 


\author{
ter verkrijging van \\ de graad van doctor aan de Universiteit Twente, \\ op gezag van de rector magnificus, \\ Prof. dr. T.T.M. Palstra, \\ volgens besluit van het College voor Promoties, \\ in het openbaar te verdedigen \\ op donderdag 26 oktober 2017 om 16.45 uur
}

door

Arvid Quintijn Léon Keemink, geboren op 22 december 1987

te Purmerend 
Dit proefschrift is goedgekeurd door:

Prof. dr. ir. Herman van der Kooij (promotor)

Dr. ir. Arno H.A. Stienen (co-promotor)

ISBN: 978-90-365-4408-5

Copyright (C) 2017 A.Q.L. Keemink 
This work was supported by the Dutch Technology Foundation STW (H-Haptics, project number: 12162), which is part of the Dutch Organisation for Scientific Research (NWO), and which is partly funded by the Ministry of Economic Affairs.

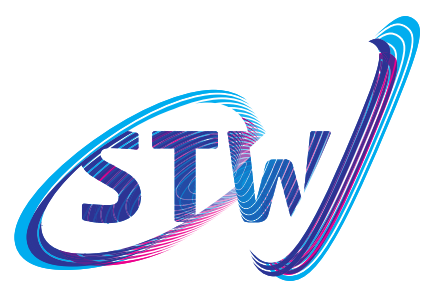

connecting innovators

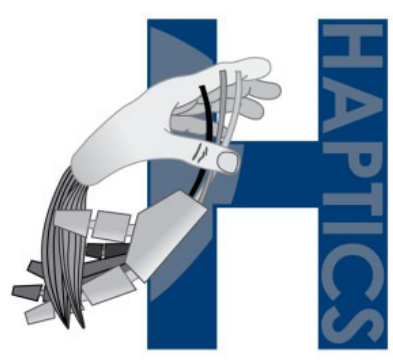

This work has also benefited from the advice and financial support of the following companies and organizations. Their support is thankfully acknowledged.

Hankamp Gears

Siza

Intespring/Laevo
WWW.HANKAMPGEARS.NL

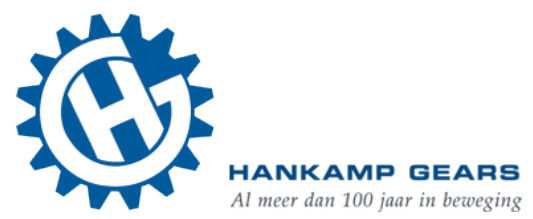

WWW.SIZA. N L

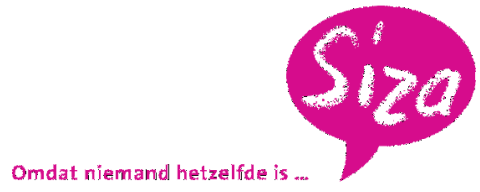

WWW.LAEVO.N L

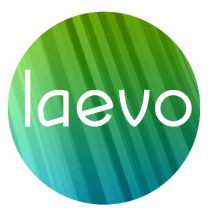



Voor mijn familie.

Careful.

We don't want to learn from this.

- Bill Watterson, Calvin and Hobbes 



\section{CONTENTS}

\section{SUMMARY}

1 GENERAL INTRODUCTION 21

1.1 The Need for Physical Assistance . . . . . . . . . . . . . . . 21

1.2 Exoskeletons for Physical Assistance . . . . . . . . . . . . 22

1.3 The H-Haptics Project. . . . . . . . . . . . . . . . 23

1.4 Research Opportunities and Research Questions . . . . . . . . . . 25

1.5 Dissertation Outline and Scientific Contributions . . . . . . . . . . 27

I MOTION \& KINEMATICS: SUPPORTING THE HUMAN SHOULDER 31

2 VISUALIZATION OF SHOULDER RANGE OF MOTION FOR CLINICAL DiAgNOSTICS AND DEVICE DEVELOPMENT 33

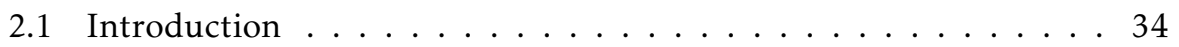

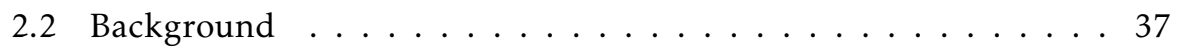

2.3 Method ...................... . . 40

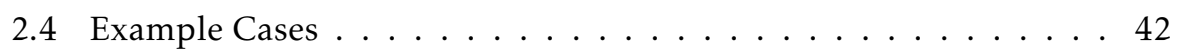

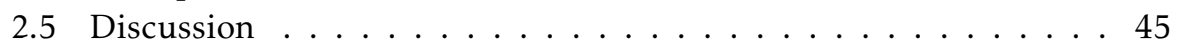

3 DifFERENTIAL INVERSE KINEMATICS OF A REDUNDANT 4R EXOSKELETON SHOULDER JOINT

3.1 Introduction . . . . . . . . . . . . . . . . 49

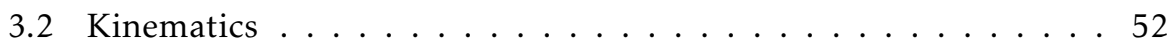

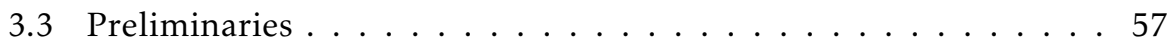

3.4 Proposed Inverse Kinematics Method . . . . . . . . . . . . . . . 65

3.5 Results ....................... 74

3.6 Discussion and Conclusion . . . . . . . . . . 78

II HAPTICS \& CONTROL: ADMITTANCE CONTROL 81

4 ADMitTANCE CONTROL FOR PHYSICAL huMAN-ROBOT INTERACTION 83

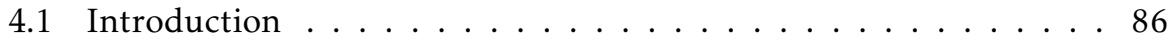

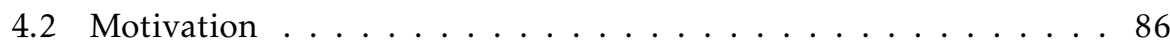

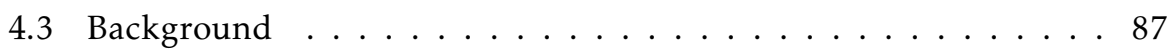

4.4 Stability and Passivity . . . . . . . . . . . . . . . . . . . 92

4.5 Admittance Control Model . . . . . . . . . . . . . . . . . . 97 
4.6 Guidelines for Minimal Inertia . . . . . . . . . . . . . . . . . . 108

4.7 Discussion . . . . . . . . . . . . . . . . 120

5 SPIS: SWITCHING DAMPING ADMITTANCE CONTROL FOR STABLE INTERACTION WITH INERTIA REDUCTION 123

5.1 Introduction . . . . . . . . . . . . . . 126

5.2 Energetic Behavior . . . . . . . . . . . . . . . . . . 129

5.3 Stability and Passivity Conditions for Admittance Control . . . . . 138

5.4 Linear Dynamical Analysis of SPIS . . . . . . . . . . . . . . . . 140

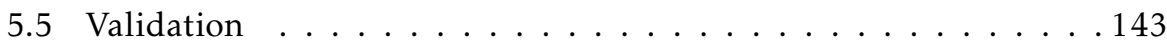

5.6 Results . . . . . . . . . . . . . . . . . . 146

5.7 Discussion . . . . . . . . . . . . . . . . . . 149

5.8 Conclusion . . . . . . . . . . . . . . . . 152

III SHARED CONTROL: DAMPING FORCES AROUND REACHING TARGETS 155

6 USING POSITION DEPENDENT DAMPING FORCES AROUND REACHING TARGETS FOR TRANSPORTING HEAVY OBJECTS: A FITTS' LAW $\begin{array}{ll}\text { APPROACH } & 157\end{array}$

6.1 Introduction . . . . . . . . . . . . . . . . 159

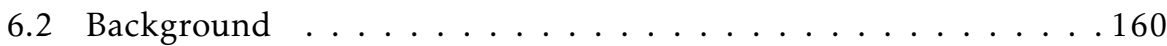

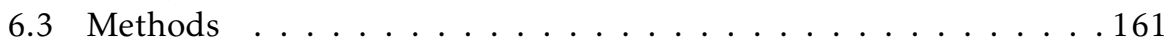

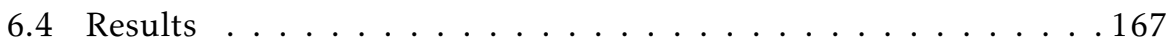

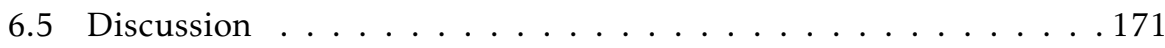

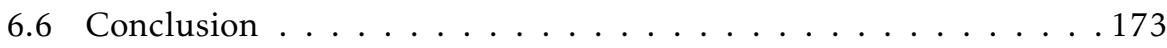

7 RESISTANCE IS NOT FUTILE: HAPTIC DAMPING FORCES MITIGATE EFFECTS OF MOTOR NOISE DURING REACHING 175

7.1 Introduction . . . . . . . . . . . . . . 177

7.2 Dynamics and Optimal Control of the Task . . . . . . . . . . . 181

7.3 Comparison of Results . . . . . . . . . . . . . . . . 185

7.4 Discussion and Future Work . . . . . . . . . . . . . . 191

IV GENERAL CONCLUSIONS

8 DisCUSSION 199

8.1 Supporting the Range of Motion of the Human Shoulder . . . . . . 199

8.2 Implementing Stable Admittance Control with Inertia Reduction . 204

8.3 How Humans Respond to Dissipative Shared Control Forces . . . 209

8.4 Overall Conclusions . . . . . . . . . . . . . . . 212 
$\begin{array}{llr}\text { V APPENDIX } & 233\end{array}$

A APPENDIX TO CHAPTER 2

B APPENDIX TO CHAPTER $3 \quad 237$

C APPENDIX TO CHAPTER $4 \quad 241$

D APPENDIX TO CHAPTER 5

ACKNOWLEDGMENTS

BIOGRAPHY 265

$\begin{array}{ll}\text { PUBLICATIONS } & 267\end{array}$ 

Physical assistance of humans implies the artificial increase of human force output and mechanical power output by means of assistive devices. Such assistance is needed if the human body is unable to perform, or repeat, a task due to physical inability, loss of limb function or fatigue.

Exoskeletons are passive or active robotic devices that are worn around the upper-extremities, the lower-extremities, trunk or neck. These devices deliver physical assistance and can increase the strength and endurance of the human wearer. The use of active exoskeletons gives the possibility of applying sophisticated methods of control that make an exoskeleton useful for a wide range problems where physical assistance is required. Robotic intelligence can help the user by intentionally shaping the interaction dynamics, i.e. the haptics, of the exoskeleton itself and the environment it interacts with. By masking friction forces, gravity forces and mechanical inertia of large devices it becomes possible to reduce the user's physical effort to perform physically demanding tasks.

In this dissertation we present several improvements for active exoskeletons for physical human-machine interaction in an attempt to make especially upperextremity exoskeletons more versatile and applicable.

To tackle several problems faced in exoskeleton design we investigated three research questions:

Kinematics \& motion: How to support the full range of motion of the human shoulder? We present a novel visualization method to display and communicate the rotational range of motion (ROM) of both the human shoulder girdle and devices that possibly support such motions. The human shoulder ROM is commonly presented along five medical directions. This neglects any coupling between these directions. Our method is based on the fact that the rotation of the upper arm can be decomposed into three angles that are displayed on a discrete grid in a Mollweide map projection. This makes it possible to evaluate and compare human and device ROM. Also quantitative device properties, such as joint conditioning or dynamical parameters, can be readily displayed and interpreted. The visualization provided by this method enables one to improve the shoulder exoskeleton design that would fit the human's ROM, kinematic properties and dynamical properties. 
Additionally, we present the differential inverse kinematics solution for a novel four degree of freedom exoskeleton to assist the full range of motion of the human shoulder. Compared to conventional exoskeletons, an additional, fourth, actuated degree of freedom is used to achieve one degree of freedom of movement redundancy around the shoulder. This redundancy is exploited to avoid kinematic singularity, and body and limb collision. Redundancy resolution is performed through nullspace motions, without influencing the movement of the arm. Instead of gradient projection of some secondary objective, feedback along the one-dimensional self-motion-manifold steers the exoskeleton towards preferred configurations. The proposed inverse kinematics method, together with such a four degree of freedom exoskeleton, allow for collision-free and singularity-free motions of the arm that would otherwise not have been possible with conventional three degree of freedom designs.

Haptics \& Control: How to get devices such robots or exoskeletons to behave as some defined impedance in a stable manner when interacting with human users; how to implement stable admittance control with inertia reduction? We present an analysis of admittance control as an interaction control method and give a set of seven guidelines how to implement admittance control to achieve apparent inertia reduction for the user. The design guidelines are derived from a desire for device passivity; dynamical behavior in which the device does not deliver an excessive amount of energy back to the human operator. In this way, safety and stability can be guaranteed during physical human-robot interaction.

Furthermore, we present the main origin of the admittance controller's inherent active behavior. From this analysis we derive a passivity inspired variable virtual damping controller for admittance control that stabilizes interaction environments characterized mainly by stiffness. By keeping track of the stored energy in the virtual model dynamics and the exchanged energy between the device and the human operator, a variable damper is switched on or off in the virtual model. Although passivity cannot be achieved with this controller, nearly passive and stable behavior can be guaranteed.

Human Factors: How do humans respond to dissipative shared control forces? We present the evaluation of a simple haptic shared controller based on position dependent damping forces. These damping forces act on the user's hands or arms during fast reaching movements. Such reaching movements represent, for example, transportation movements performed by workers in real-world industrial production processes or transportation of heavy objects. Simple sensors can gather limited environment information and the shared controller applies these 
damping forces to assist the user in accurate placement and faster movements towards task related targets. By assisting goal directed movements with damping around reaching targets, it is shown that humans increase their reaching accuracy as well as decrease their movement times, when compared to free-air conditions. The relation between increasing end-point accuracy and distance and increasing movement time, known as Fitts' Law, is shown to hold for these dynamical conditions as well. It is hypothesized that damping forces mitigate the mechanical effects of activation dependent, or multiplicative, motor noise. The attenuation of the effects of noise on end-point accuracy allows for higher muscle forces and accelerations, while still guaranteeing the requested accuracy. Nonlinear stochastic optimal control models show agreement with measured data. This supports the hypothesis that humans preform their reaching movements while optimizing for energy efficiency and minimizing variance to an amount that the task requires.

This research showed that haptic shared control can be passive and as simple as damping around an estimated reaching target. This is an addition to the more common methods of assistive and repulsive potential forces used in haptic guidance, or mechanical constraints imposed in passive Cobot systems. Such damping forces possibly allow for haptic guidance like behavior on completely passive assistive devices. 

Technische hulpmiddelen staan versterking van menselijke kracht en menselijk mechanisch vermogen toe. Zulke hulpmiddelen zijn nodig wanneer het menselijk lichaam niet in staat is om nuttige taken uit te voeren of te herhalen, bijvoorbeeld vanwege fysiek onvermogen, verlies van functie of door vermoeiing.

Exoskeletten zijn passieve of actieve robotische hulpmiddelen die gedragen kunnen worden om de bovenste extremiteiten, de onderste extremiteiten, romp of nek. Deze hulpmiddelen leveren fysieke ondersteuning versterken zo de kracht of verhogen het uithoudingsvermogen van de drager. Het gebruik van actieve exoskeletten geeft de mogelijkheid om geavanceerde regelmethoden te gebruiken. Zulke methoden maken exoskeletten inzetbaar voor een breed scala aan problemen waarbij fysieke ondersteuning nodig is. Robotische intelligentie kan de drager helpen door het aanpassen van de interactiedynamica, ofwel de 'haptics'. Dit kan beïnvloeden hoe het exoskelet zelf aanvoelt, of hoe de fysieke omgeving waar de drager interactie mee wordt waargenomen door de drager van het exoskelet. Door het onderdrukken van wrijving in de aandrijving en het reduceren van zwaartekracht en mechanische traagheid van exoskeletten wordt het mogelijk om de moeite die de gebruiker moet doen te verminderen en diens vermogen om zware fysieke taken te doen te vergroten.

In dit proefschrift presenteren we een aantal verbeteringen voor actieve exoskeletten die gebruikt kunnen worden voor fysieke mens-machine interactie. Het doel van dit werk is om exoskeletten voor de bovenste ledematen meer veelzijdig en toepasbaar te maken.

Het onderzoek werd afgeleid uit drie problemen die spelen binnen exoskeletontwerp:

Kinematica \& beweging: Hoe kan het volledig bewegingsbereik van de menselijke schouder worden ondersteund? We presenteren een nieuwe methode om het rotationele bewegingsbereik van zowel de menselijke schouder als ondersteunende hulpmiddelen voor de schouder weer te geven en beter te communiceren in publicaties. Het rotationele bewegingsbereik van de menselijke schouder is doorgaans uitgedrukt in het bereik van vijf medische richtingen. Het communiceren van rotationeel bewegingsbereik langs deze vijf richtingen negeert echter elke vorm van koppeling, en daarmee bijkomende beperkingen in bereik, 
tussen deze richtingen. Onze methode is gebaseerd op het feit dat elke rotatie van de bovenarm kan worden ontbonden in drie principiële hoeken. Deze hoeken worden weergegeven op een discreet rooster in een Mollweide bol-projectie. Dit maakt het mogelijk om het bewegingsbereik van de menselijke schouder en van ondersteundende hulpmiddelen te vergelijken. Ook kunnen kwantitatieve eigenschappen, zoals kinematische conditionering van een gewricht of dynamische parameters van een ledemaat of hulpmiddel direct worden weergegeven, geïnterpreteerd en vergeleken. De weergavemethode kan gebruikt worden bij exoskeletontwerp om beter rekening te kunnen houden met nuttig en veilig werkbereik van de schouder, te bepalen of een hulpmiddel niet gevaarlijk buiten het bewegingsbereik treedt of te restrictief is.

Verder presenteren we een differentile oplossingsmethode om het inversekinematicaprobleem op te lossen voor een schouderexoskelet met vier vrijheidsgraden. Dit exoskelet zou het hele rotationele bewegingsbereik van de menselijke schouder moeten kunnen bereiken zonder last te hebben van kinematische singulariteit of botsing met het lichaam. Ten opzichte van een conventioneel ontwerp is een extra, vierde, geactueerde vrijheidsgraad toegevoegd. Dit zorgt voor één redundante vrijheidsgraad in beweging rond de schouder. Deze redundantie wordt uitgebuit om kinematische singulariteiten en botsingen met he lichaam te voorkomen. De redundantie wordt opgelost via bewegingen in de nullruimte van de geometrische Jacobiaan, welke geen invloed kunnen hebben op de beweging van de menselijke arm. In plaats van het gebruiken van gradiëntprojectie van een secundair doel gespecificeerd in gewrichtsruimte, gebruiken we terugkoppeling langs de ééndimensionale variëteit van interne bewegingen. Op deze manier wordt het exoskelet naar een meer gunstige configuratie gestuurd, zonder de menselijke arm te beïnvloeden. De voorgestelde methode samen met de vierde vrijheidsgraad staan toe een exoskelet te ontwerpen dat singulariteit- en botsingvrij is.

Haptics \& Control: Hoe kunnen we apparaten zoals robots en exoskeletten zich stabiel laten gedragen als een bepaalde impedantie tijdens interactie met menselijke gebruikers; hoe kan admittance control worden geïmplementeerd om traagheidsreductie te garanderen? We presenteren een analyse van admitance control als een methode voor interactieregeling voor robots. Zeven richtlijnen beschrijven hoe admittance control kan worden gebruikt om de waargenomen mechanische traagheid van robots te verlagen. Deze richtlijnen zijn afgeleid uit de wens voor energetisch passief dynamisch gedrag van de robot; dynamisch gedrag waarbij de robot nooit meer energie kan leveren aan de mens dan de menselijke gebruiker 
ooit aan de robot heeft geleverd. Op deze manier kunnen stabiliteit en veiligheid van de robot gegarandeerd worden tijdens fysieke interactie tussen mens en robot.

Verder presenteren we de oorzaak van het inherent actieve gedrag van een typische admittance controller. Uit deze analyse leiden we een op passiviteit geïnspireerde controller af. Deze controller, effectief een variabele demper, stabiliseert de interactie van robots met stijve omgevingen. Door de opgeslagen energie in het virtuele model en de energie uitgewisseld tussen mens en robot bij te houden kan deze demper aan- of uitgeschakeld worden in het virtuele model. Alhoewel passiviteit niet kan worden gegarandeerd met deze regelaar, is het dynamisch gedrag bijna passief.

Human Factors: Hoe reageren mensen op dissipatieve shared control krachten? We presenteren de evaluatie van een simpele haptic shared controller, welke gebaseerd is op positieafhankelijke dempingskrachten. Deze controller remt de beweging van een menselijke hand af tijdens een snelle doelgerichte reikbeweging. Zulke reikbewegingen zijn representatief voor, bijvoorbeeld, bewegingen die uitgevoerd worden tijdens het transport van zware producten of goederen bij industriële productieprocessen. Beperkte informatie van simpele sensoren kan de omgeving grof in kaart berngen, en de shared controller kan vervolgens aan de hand van deze informatie dempingskrachten leveren om de mens te sturen en deze nauwkeuriger objecten te laten plaatsen. We laten zien dat wanneer positieafhankelijke demping wordt gebruikt, mensen sneller en nauwkeuriger bewegen dan tijdens een reikbeweging in de vrije lucht. De relatie tussen de uiteindelijk nauwkeurigheid en de bewegingsafstand en de duur van de beweging is ook wel bekend als de 'wet van Fitts'. We laten zien dat deze wet ook geldt voor bewegingen die beïnvloed worden door positieafhankelijke demping. Onze hypothese is dat dempingskrachten de mechanische effecten onderdrukken van activatieafhankelijke, of multiplicatieve, motorruis van het menselijk bewegingsapparaat. De onderdrukking van de effecten van ruis op de reiknauwkeurigheid staat de mens toe hogere spierkracht en versnellingen te genereren met meer ruis, terwijl de benodigde nauwkeurigheid nog kan worden gegarandeerd. Niet-lineaire stochastische optimal control modellen laten overeenkomsten zien met de gemeten data. Dit ondersteunt de hypothese dat mensen hun reikbeweging optimaal uitvoeren in een afweging om energie-efficiënt en nauwkeurig genoeg voor de taak te zijn.

Dit onderzoek laat zien dat een haptic shared controller passief kan zijn, en zo simpel als dempingskracht rondom een geschat bewegingsdoel. Dit is een toe- 
voeging aan de meer gangbare shared controllers die gebaseerd zijn op helpende potentiële krachtvelden of mechanische beperkingen opgelegde in passivie Cobot systemen. Dempingskrachten staan toe haptic-guidance-achtig gedrag te genereren op compleet passive hulpmiddelen. 
Physical assistance of humans implies the artificial increase of human force output and mechanical power output by means of assistive devices. Such assistance is needed if the human body is unable to perform, or repeat, a task due to physical inability, loss of limb function or fatigue.

\subsection{THE NEED FOR PHYSICAL ASSISTANCE}

In the Netherlands, physically demanding labor is required by $42 \%$ of the workforce. Specifically in health care and in industrial sectors, respectively $46 \%$ and $47 \%$ of the workers reported performing physically demanding tasks in 2015 [1].

About $85 \%$ of the Dutch healthcare workers report musculoskeletal disorders due to heavy lifting [2]. Unassisted heavy lifting of patients between bed and wheel-chair and repositioning patients for daily tasks put serious mechanical strain on the arms and back of caregivers. In the coming decades this problem will become more prevalent; more elderly will require medical care, with fewer medical professionals available to provide it [3].

The majority of production processes in industry cannot be fully automated. Requirements for intelligence and flexibility or small product batch sizes make such a feat technologically challenging and prohibitively expensive. In the EU, human industry workers are still required during production processes. Unfortunately, they suffer from high physical workload due to heavy material lifting and handling (30\% of the work population), movement repetition (63\%) and awkward body posture (46\%). This overloading of the body results several prevalent musculoskeletal disorders. More than $40 \%$ of the EU workers report lower back pain, or neck and shoulder pain [4].

The Dutch 'Stichting Zorg en Welzijn' (SZW), and European guidelines [5] enforce an upper limit on the amount of physical loading due to lifting, which is based on recommendations from the US National Institute for Occupational Safety and Health (NIOSH) [6]. It recommends manual lifting of maximally 23 $\mathrm{kg}$ under the most ideal circumstances. Lifting of patients and materials above this maximal amount is associated with an increased risk in lower back injury 
due to excessive spinal loads [7, 8]. Lifting or carrying weights as low as $5 \mathrm{~kg}$ for many hours daily, increases the risk of work related lower back problems [5].

The estimated yearly cost due to labor induced neck and back pain in the Netherlands was estimated to be around 1.3 billion Euros for health care costs [9], with an additional loss of productivity and service of 800 million Euros in 2013 [10]. Therefore, devices and methods that support lifting and correct body posture for targeted worker groups can have significant benefit to national health, productivity and welfare.

\subsection{EXOSKELETONS FOR PHYSiCAL ASSISTANCE}

Rules and regulations attempt to enforce specific methods of lifting or to use tools to reduce the load. Technical solutions such as cranes, (sling) lifts and trolleys are used, but are found to be bulky and cumbersome, limited in capability or not readily available at the required location under time pressure.

A recent development to overcome some of these problems is the use of exoskeletons $[11,4]$ that are worn during most the working day and move with the body of the user. Exoskeletons are passive or active robotic devices that are worn around the upper-extremities (arms), the lower-extremities (legs), trunk or neck. These devices deliver physical assistance and can increase the strength and endurance of the human wearer. In contrast to end-effector assistive devices, exoskeletons are fully (or mostly) anthropomorphic in the sense they follow the kinematics of the human body and limbs [12].

The use of active exoskeletons gives the the possibility of applying sophisticated methods of control that make an exoskeleton useful for a wide range problems where physical assistance is required. Robotic intelligence can help the user by intentionally shaping the interaction dynamics of the exoskeleton itself and the environment it interacts with. A controller that explicitly shapes the robot dynamics to feel like some predefined impedance to the human user is called a haptic controller. By masking friction forces, gravity forces and masking mechanical inertia of large devices the controlled exoskeleton reduces the user's physical effort for physically demanding tasks. Such methods effectively amplify the user's force, thereby reducing inertia of the robot or the robot-limb combination [13].

Exoskeletons that are passive are useful as arm supports for patients with weakness or for supporting awkward and fatiguing arm posture during industrial applications. Furthermore, passive exoskeletons can be preferred in cases 


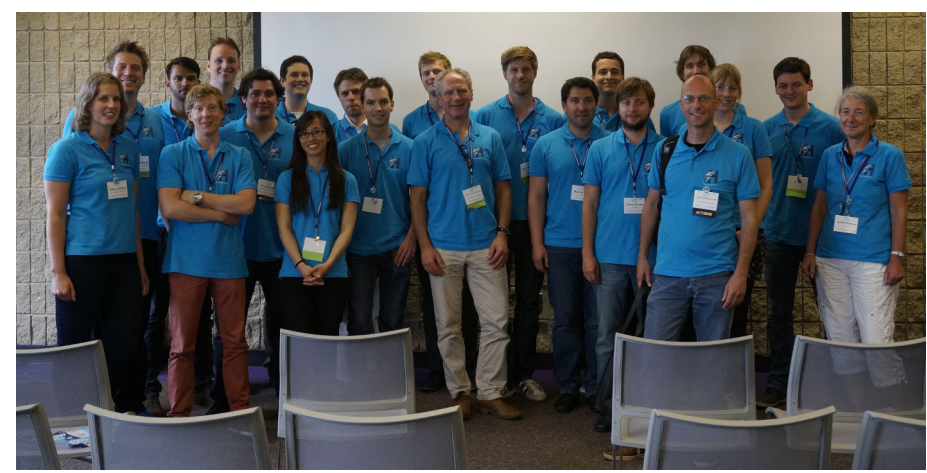

Figure 1.1: A large fraction of the H-Haptics research consortium at World Haptics Conference 2015 at Northwestern University, Chicago, USA.

where requirements for portability, a small form factor and low power usage do not allow for a fully actuated system.

Many reported exoskeletons and arm supports are still at an experimental stage and not ready to be used in practice [4]. Although many exoskeletons have been developed to be used in industry [4] and a greater number has been developed for upper-extremity rehabilitation $[14,15]$, the amount of commercially available exoskeletons is low. This is because several technical limitations need to be overcome. Device kinematics are currently not optimal to support full human range of motion, interaction control methods do not deliver a transparent method of physical exoskeleton control and more knowledge is required about how humans respond to such control methods and how the applied forces can be optimally shaped.

In this dissertation we tackle several of these problems. We develop several improvements for active exoskeletons for physical human-machine interaction in an attempt to make upper-extremity exoskeletons more versatile and applicable. The motivation for this work originally came from the $\mathrm{H}$-Haptics research project.

\subsection{THE H-HAPTICS PROJECT}

The H-Haptics research project [16] (see Fig. 1.1) aimed at developing a deeper understanding of how humans interact with devices that provide force feedback. Typical applications of such devices are in the field of tele-operation, used e.g. 
for remote maintenance, deep sea mining and dredging or surgery. In such scenarios a user moves a haptic master device, while a slave robot follows these movements at a remote location. All forces that the slave robot experiences at the remote location are in some way fed back to the master device to be felt by the user. Proper control of such devices can be developed to make the masterslave combination approximately as 'haptically transparent' to the user as possible; as if the system is not there and the user interacts directly with the remote environment.

It has been argued that perfect transparency is not necessarily a design requirement for teleoperation systems. Human force sensing capabilities are limited, and the operator is usually more interested in performing the task well or quickly. An intelligent system that continually shares the control authority with the human is called a haptic shared controller $[17,18]$. During shared control, the human operator stays in manual control while the controller provides continuous support, or opposition, by applying forces on the master device. For example, with poor transparency during bilateral tele-operation, a shared controller can have a positive effect on task completion time and performance [19].

Shared control is a control method on the edge of full automation and humanonly task performance. In many real-world tasks full automation is not yet possible due to the lack of intelligent adaptability of devices that humans do have. Therefore, human-in-the-loop solutions are still required. During shared control a controller provides forces on the master device or changes its impedance to assist the user or to inform user about how the controller 'believes' the user should act and perform a task.

Within the total H-Haptics project, our project focused explicitly on haptic physical assistance of humans, especially heavy lifting, with exoskeletons. This work-package comprised two PhD students for research and one PDEng trainee for device development. Jack Schorsch, a PhD from Delft Technical University in the Netherlands, focused on device design and human factors experiments. Next to designing interaction mechanics for a supportive exoskeleton [20], he investigated how humans respond to physical interactions with systems that have a mismatch in weight and inertia when a device between the user and the load filters haptic information [21].

Michel van Hirtum, a PDEng trainee at the University of Twente in the Netherlands, designed the mechanics and mechatronics of a semi-passive gravity support device that can be part of an exoskeleton or otherwise mobile system. 


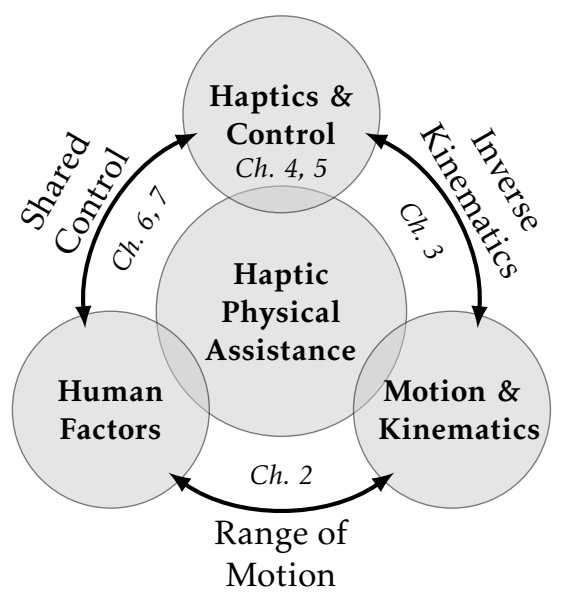

Figure 1.2: Haptics \& Control, Human Factors and Motion \& Kinematics are three pillars in the design of exoskeletons for physical interaction. The work in this dissertation falls under (combinations of) these three categories. The chapters in this dissertation (denoted by Ch.) are placed near their relevant pillar or connection between pillars.

I, also at the University of Twente, focused on fundamental issues in haptic physical human assistance; how to optimize haptic interaction control and make it safe, improving exoskeleton kinematics and the use of rudimentary shared control during assisted lifting. The main focus of the work was to overcome several current problems in haptic exoskeleton design. The work presented in this dissertation is the culmination of four and a half years of this research.

\subsection{RESEARCH OPPORTUNITIES AND RESEARCH QUESTIONS}

To tackle some of the current problems faced in haptic exoskeleton design we investigated three fundamental pillars that lead to several research opportunities. These pillars are shown in Fig. 1.2, linking them to the six chapters in this dissertation. The research opportunities are as follows:

I. Kinematics \& motion: How can we get devices to move with humans along their full range of motion?

For the upper extremity, the shoulder is the most complex joint. Device development and kinematic measurements of rotational range of 
motion of the shoulder inherently suffer from the artificial distinction of the five medical definition of directions of motion. This makes it difficult to properly design exoskeleton range of motion that fully covers the human range of motion. Furthermore, conventional three degree of freedom exoskeleton designs that attempt to support this range of motion fail due to body collisions and prevalent kinematic singularity.

II. Haptics \& Control: How to get admittance controlled devices, such robots or exoskeletons, to behave as some defined impedance in a stable manner when interacting with human users?

Admittance control is a haptic interaction control paradigm that uses measurements of interaction force between a robot and a human to generate a motion reference for the robot to follow. This motion reference is derived from a virtual dynamical model that resembles the target impedance the human is supposed to feel during interaction. A benefit of admittance control over more conventional impedance control is that it allows a human to physically control heavy robots and assistive devices that have high inertia, high weight, high friction and high transmission ratios. When admittance control attempts to reduce the inertia of the robot and a possible carried load, this control method might make the robot unstable when it is in contact with a human limb.

III. Human Factors: How do humans respond to dissipative forces?

Much of the shared control literature focuses on guidance forces based on potential (stiffness-like) fields that push or pull the user toward some point or onto some trajectory. We reason that there could be potential for dissipative shared control where damping forces steer the user. The benefit of damping forces is that they are fully passive and are therefore inherently safe, and could also be implemented in passive lifting devices and arm support devices. We currently do not know how humans respond to damping forces and in what form they are beneficial for a specific task.

These three research opportunities were specified to three fundamental research questions that are related to the aforementioned problems: 
Research Questions

I. How to support the full range of motion of the human shoulder?

II. How to implement stable admittance control with inertia reduction?

III. How do humans respond to dissipative shared control forces?

These three main research questions separate this dissertation into three major parts, as is explained in more detail in the following section.

\subsection{DisSERTATION OUTLINE AND SCIENTIFIC CONTRIBUTIONS}

The chapters in this dissertation, excluding the introduction and discussion, were written as journal or peer-reviewed conference publications. This causes some overlap between some chapters, but allows the reader to read most of them individually or out of order. Many chapters do not focus on a solution for exoskeletons specifically, because the work presented is more generally applicable.

Part I: Motion \& Kinematics: Supporting the Human Shoulder. This part focuses on the range of motion of the human shoulder girdle and an accompanying conceptual four degree of freedom kinematic exoskeleton design for a shoulder joint.

Chapter 2 presents a novel visualization method to display and communicate the rotational range of motion (ROM) of both the human shoulder girdle and devices that possibly support such motions. The ROM of the human shoulder is commonly presented along five medical directions of motion. This neglects any coupling in ROM between these directions, which are henceforth also neglected in exoskeleton and device design. Compared to existing methods, our method is based on the fact that the rotation of the upper arm can be decomposed into three angles that are displayed on a discrete grid in a two-dimensional Mollweide map projection. This avoids the need to show several three-dimensional figures in two-dimensional print to show and communicate the shoulder ROM. This makes it possible to evaluate human ROM, to evaluate visually how the ROM coupled between movement directions, evaluate influence of pathologies and their recovery on shoulder ROM, and make comparisons between the ROM between humans and de- 
vices. The method also allows to show quantitative device properties such as joint conditioning (displaying kinematic singularity) and dynamical parameters, or human movement properties such as forces, movement times and targeting variance.

Chapter 3 presents a differential inverse kinematics (IK) solution method of a novel four degree of freedom (4R) exoskeleton design to assist the full range of motion of the human shoulder. An additional, fourth, actuated degree of freedom is used to achieve one degree of freedom of movement redundancy. This redundancy is exploited to avoid kinematic singularity, and body and limb collision. Redundancy resolution is performed through nullspace motions, without influencing the movement of the arm. Instead of gradient projection of some secondary objective, feedback along the one-dimensional selfmotion-manifold steers the exoskeleton towards more preferred configurations. Compared with existing three degree of freedom methods this method allows for a larger, singularity-free workspace of the exoskeleton joint. Compared with a recently published other $4 \mathrm{R}$ exoskeleton that used a non-differential IK method, our method optimizes to find a kinematically more favorable configuration on-line, even while the human arm is stationary.

Part II: Haptics \& Control: Admittance Control. This part focuses on admittance control. Admittance control is an interaction control method that relies on force measurement from the user. The user's force is effectively amplified by calculating an exoskeleton reference motion through a virtual dynamical model. The exoskeleton moves according to the model, and the apparent mechanical impedance felt by the user approaches that of the virtual model. The stability of such a control method during physical interaction with human users is not trivial. Especially when the device is being held by a user this interaction method can become unstable if it attempts to reduce the inertia of the device and carried loads. The advantage of using admittance control over impedance control is that it allows a human to physically control heavy robots and assistive devices that have high inertia, high weight, high friction and high transmission ratios while simulating damping and varying stiffness values in a stable way, required for assistive tasks.

Chapter 4 presents an analysis of admittance control as an interaction control method, and provides a new set of guidelines on how to implement admittance control to achieve apparent inertia reduction for the user. The design guidelines are derived from a desire for device passivity; dynamical behavior where the device can deliver no excessive amount of energy back 
to the human operator. In this way, safety and stability can be guaranteed during physical human-robot interaction.

Chapter 5 presents the cause of active behavior of naive admittance control implementations. It shows why recently proposed methods to attempt timedomain passivity control cannot be used to reduce the apparent device inertia. Our work presents a different stabilization method: a passivity inspired variable virtual damping controller for admittance control that stabilizes interaction with stiffness-like environments. By keeping track of the stored energy in the virtual model dynamics and the exchanged energy between the device and the human operator, a variable damper is switched on or off in the virtual model. Although passivity cannot be achieved, nearly passive and stable behavior can be guaranteed.

Part III: Shared Control: Damping Forces around Reaching Targets. This part focuses on a simple form of shared control: applying damping forces to a device and user during reaching movements. Such reaching movements represent transportation movements performed by workers in real-world settings. Simple sensors can gather limited environment information and the shared controller applies these damping forces to assist the user in accurate placement and faster movements towards task related targets.

Chapter 6 presents the evaluation of a novel simple haptic shared controller based on damping forces. By assisting goal directed movements with damping around reaching targets, it is shown that humans increase their reaching accuracy as well as decrease their movement times, when compared to free conditions. The relation between increasing end-point accuracy and distance and increasing movement time, known as Fitts' Law, is shown to hold for these dynamical conditions as well.

Chapter 7 presents an addendum to Chapter 6. Here, the evidence for how human subjects are able to decrease reaching time while subjected to longitudinal haptic damping forces is discussed. We state the hypothesis that damping forces mitigate the mechanical effects of activation dependent (or multiplicative) motor noise. The attenuation of these effects of noise on endpoint accuracy allows for higher muscle forces and accelerations, while still guaranteeing the requested accuracy. Nonlinear stochastic optimal control models show agreement with measured data. This supports the hypothesis that humans preform their reaching movements while optimizing for energy efficiency and minimizing variance to an amount that the task requires. 



\section{Part I}

MOTION \& KINEMATICS:

SUPPORTING THE HUMAN SHOULDER 

Burdens are for shoulders strong enough to carry them.

— Margaret Mitchell, Gone with the Wind, 1936

Abstract-Rotations of the humerus in the human shoulder girdle are usually described using five classical medical definitions: flexion/extension, abduction/adduction, internal/external rotation, horizontal abduction/adduction, and horizontal flexion/extension. The latter two are needed to overcome the inability of the first three to define the full range of motion. The International Society for Biomechanics recommendations reduce these to three sequential rotations around perpendicular axes: rotation of the plane of elevation (horizontal rotation), elevation, and axial rotation. We expand on this work by providing a new and intuitive visualization method to display these rotations that superimposes the axial rotation on the map projection of the reachable workspace of the elbow. We provide methods to interpret and create the visualization using direct observations. The visualization allows the immediate observation of the full rotational range of motion of the humerus and the interaction effects between these physiologically coupled rotations. Furthermore, it allows visualization of the effects of kinematic limitations of external devices such as endpoint manipulators or exoskeletons. Therefore, the new visualization method is useful for both clinical diagnostics and device development.

This chapter was published as: A.H.A. Stienen and A.Q.L. Keemink, "Visualization of shoulder range of motion for clinical diagnostics and device development", IEEE/RAS-EMBS International Conference on Rehabilitation Robotics (ICORR), 2015. Both authors contributed equally to this work. 


\begin{tabular}{ll}
\hline Symbol & Explanation \\
\hline $\mathbf{x}, \mathbf{y}, \mathbf{z}$ & vectors of principal axes \\
$X, Y, Z$ & positive directions along principal axis vectors \\
$\bar{X}, \bar{Y}, \bar{Z}$ & negative directions along axis vectors \\
$\gamma_{a}$ & axial glenohumeral rotation angle \\
$\gamma_{e}$ & glenohumeral elevation angle \\
$\gamma_{h}$ & horizontal glenohumeral rotation angle \\
\hline & \\
\hline Abbrev. & Explanation \\
\hline GH & glenohumeral \\
ISB & International Society for Biomechanics \\
ROM & range of motion \\
\hline
\end{tabular}

\subsection{INTRODUCTION}

In this work we present a novel and intuitive visualization method for displaying the kinematics of the glenohumeral $(\mathrm{GH})$ rotations of the human shoulder girdle. The visualization method displays the rotations of the girdle via superposition of the axial rotation on the map projection of the reachable workspace of the elbow. An example of the visualization is given in Fig. 2.1.

The human shoulder girdle is a complex skeletal structure with a remarkably large range of motion (ROM). Many have measured the GH rotations [22, 23, 24], using a selection of the five classical movement directions: flexion/extension, abduction/adduction, internal/external rotation, horizontal abduction/adduction, and horizontal flexion/extension (see Fig. 2.2). These classical movements are conceptually easy to understand, but the inclusion of the latter two is needed to overcome the inability of the first three to define the full range of motion. Furthermore, the rotations are defined around an immovable global joint coordinate system that makes sequential rotations around multiple of the defined axes prone to interpretation differences. To solve these problems, the International Society for Biomechanics (ISB) recommendations reduce these five classi- 


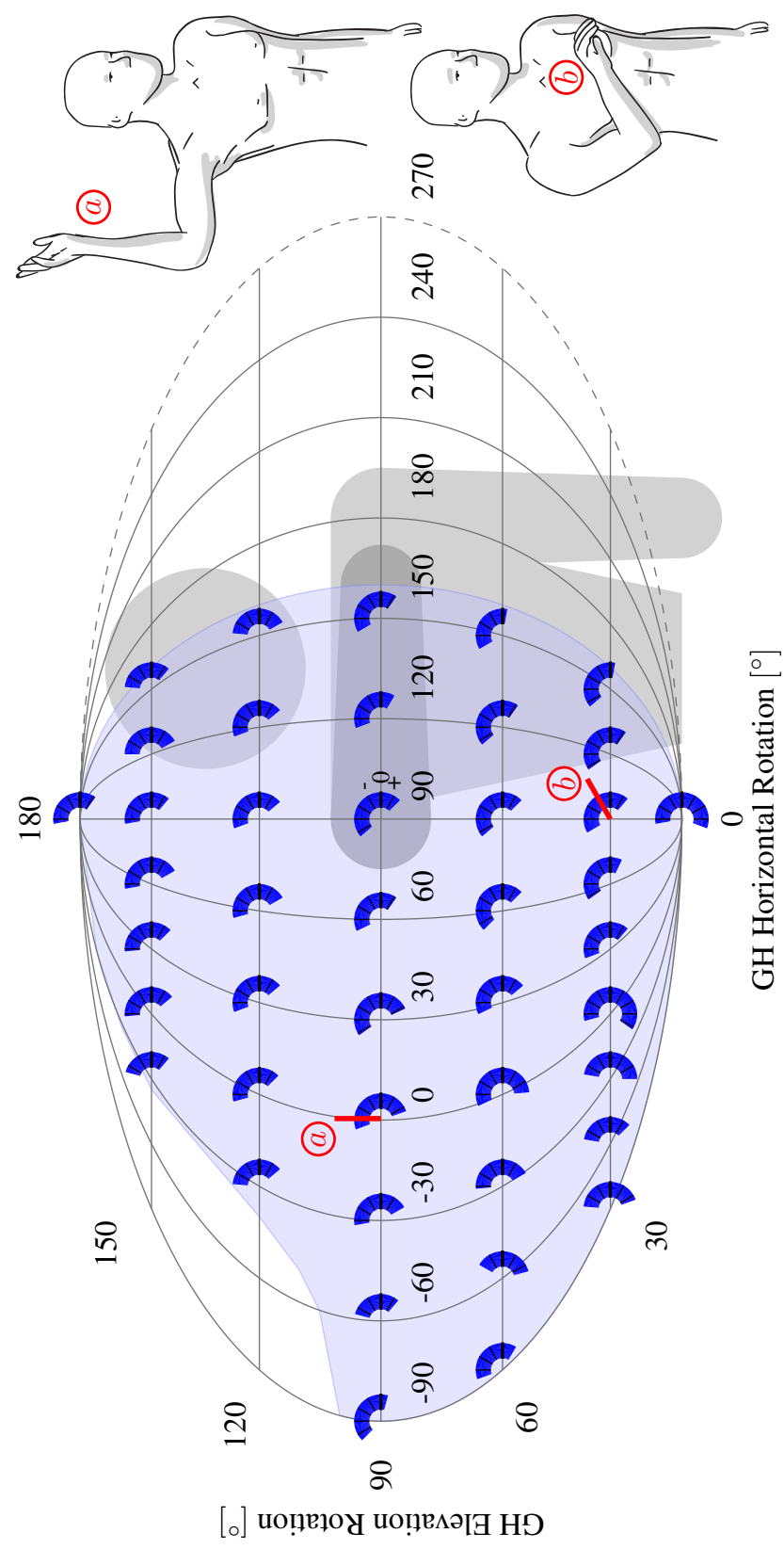

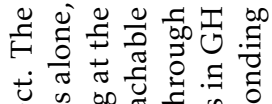

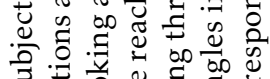

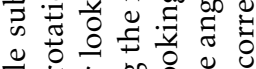

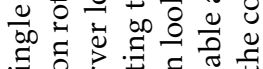
की

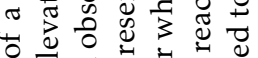

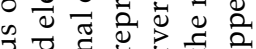
동 정 정

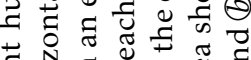

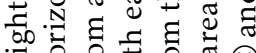

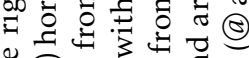

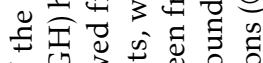
403.

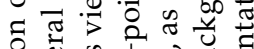
웜 ㅇ 峞 $\therefore$ 品 类 $\exists$

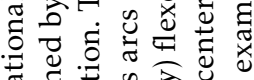

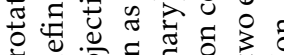
- क

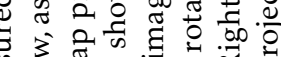
范

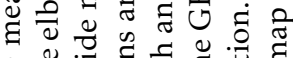
Ð फे

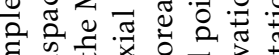
द 『ั

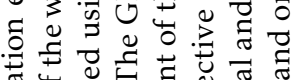

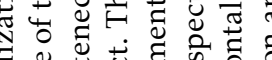

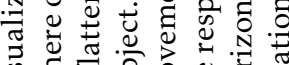

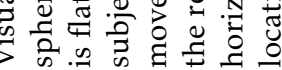
$\ddot{-}$ i 幽 


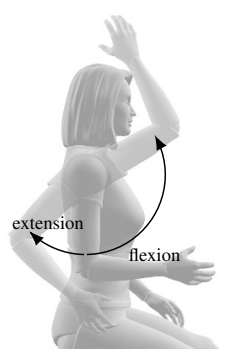

(a)

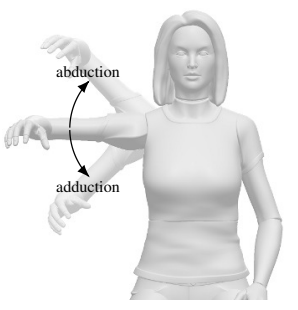

(b)

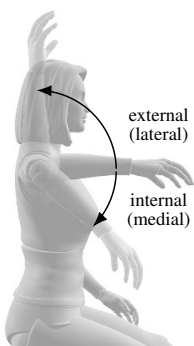

(c)

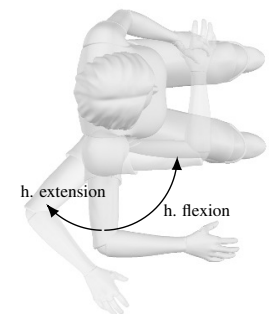

(d)

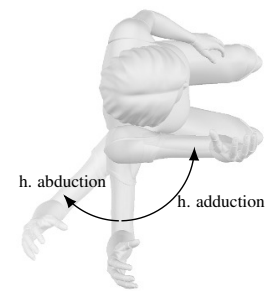

(e)

Figure 2.2: The five classical definitions of movement directions for three degrees of freedom of the shoulder: a) flexion/extension, b) abduction/adduction, c) internal/external rotation, d) horizontal flexion/extension, and e) horizontal abduction/adduction.

cal rotations to three sequential rotations around perpendicular axes [25]: GH rotation of the plane of elevation, GH elevation (negative), and GH axial rotation*. In this chapter we will use the ISB definitions but refer to them as GH horizontal rotation, $\mathrm{GH}$ elevation rotation, and $\mathrm{GH}$ axial rotation for brevity and clarity.

Independent of the joint coordinate system used, nearly all studies that report on the GH ROM give the measurements for each axis as an independent outcome measure. This ignores the interaction effects between these physiologically coupled rotations and leads to a reported ROM that is likely larger than

${ }^{*}$ As in [25], the methods presented here are valid for the right shoulder only. Whenever left shoulders are measured, it is recommended to mirror the raw position data with respect to the sagittal plane. Finally, this chapter focuses on the GH rotations only and ignores any translations of the $\mathrm{GH}$ rotation center. 
actually achievable. This might especially affect reporting in clinical diagnostics, which require the ROM to be measured and represented properly.

External interaction with the shoulder girdle is possible with a wide variety of devices, from endpoint manipulators and weight-support devices to exoskeletons and orthotics. Of these, exoskeletons are often used to directly augment the three rotations of the GH joint [26, 27, 14, 15]. Again, common presentations of human shoulder or exoskeleton shoulder ROM results are tables or graphs that indicate by numerical intervals or plotted bars the ROM along the movement directions $[22,28,29,30,31,32,33,34]$, under the assumption that these movement ranges are decoupled. In $[35,26,36,27]$ a more graphical approach is taken. They visualized the range of motion by creating a $3 \mathrm{D}$ point cloud around the human body, indicating the reachable workspace of the human arm. The third rotation, GH axial rotation, is not represented in these point clouds. The globe system [37] recognizes that a sphere of elbow positions around the humeral head is fully defined by GH horizontal and elevation rotations. It gives the same angle definitions as the ISB recommendations to decompose arm rotations onto this sphere. The axial rotation component is mainly neglected, or drawn on a front or top view of the sphere. In publication, it presents only single arm configurations using multiple top and side views, and gives therefore no information about shoulder ROM. All these presentation methods have in common that they are often hard to interpret due unintuitive representations or by attempting to show $3 \mathrm{D}$ objects in $2 \mathrm{D}$ print.

We have therefore built upon the aforementioned work an developed a new visualization method to show the complete shoulder ROM.With this method we can inspect functional ROM of patients, compare the human shoulder ROM to an exoskeleton shoulder ROM, or compare different exoskeleton shoulders. Furthermore, we will use this visualization to show kinematic or dynamic properties of exoskeleton shoulder joints.

\subsection{BACKGROUND}

\subsubsection{Kinematics of the Human Shoulder}

To geometrically describe the human shoulder, we use axes and angles definitions from the ISB [25]. As shown in Fig. 2.3, a global coordinate frame is attached to the center of rotation in the glenohumeral $(\mathrm{GH})$ rotation center of the shoulder. The global $\mathbf{x}$-axis points in the dorsoventral direction (forward), the 

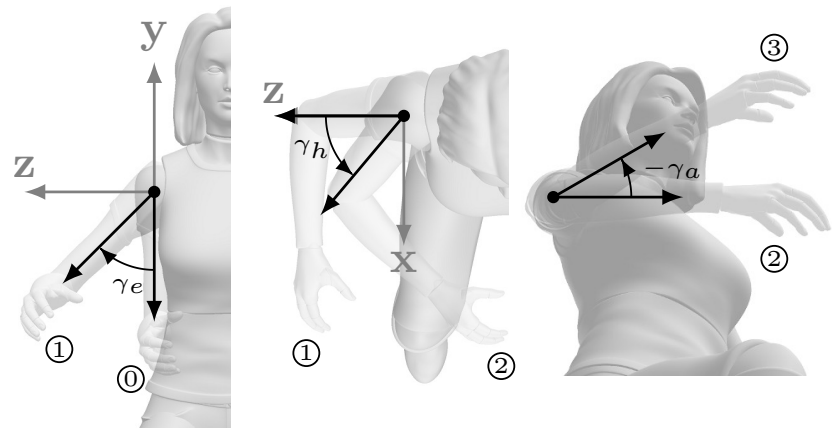

Figure 2.3: ISB Axes definitions, $\mathbf{x}$ pointing forward, $\mathbf{y}$ pointing upward and $\mathbf{z}$ pointing outward. Intuitive ISB angle decomposition from left to right; Front view: GH elevation rotation first (from pose (1) to (1), over angle $\gamma_{e}$ ). Top view: rotate horizontally (from pose (1) to (2), over angle $\gamma_{h}$ ). View perpendicular to the upper arm: finally, rotate axially (from pose (2) to (3), over angle $\gamma_{a}$ ). This decomposition is intuitively understood when performed by a person in a different order than the geometrical ISB description, due to the singular nature of their proposed $Y X Y$ rotation order

global $\mathbf{y}$-axis points in the anteroposterior direction (upward) and the global $\mathbf{z}$-axis points into the mediolateral direction (outward to the right).

The human shoulder orientation is decomposed around these axes into YXY intrinsic Euler angle rotations. These rotations are stated in the order of most to least dominant rotation, i.e. a dominant rotation also rotates a less dominant axis. Classically this $Y X Y$ decomposition would be (dominant) $Y$ : horizontal abduction/adduction, $X$ : abduction/ adduction or flexion/extension (dependent on the configuration), and (least dominant) $Y$ : internal/external rotation.

The shoulder rotates a person's upper arm, which makes the tip of the elbow cover part of the surface of a sphere (i.e. the 'elbow-tip sphere'). This sphere can be decomposed into spherical coordinates: the GH horizontal rotation $\gamma_{h}$ and $\mathrm{GH}$ elevation rotation $\gamma_{e}$ (the first two most dominant $Y X$ rotations). The third and least dominant upper arm rotation, GH axial rotation, is described directly by $\gamma_{a}$, relative to the horizontal plane. Positive GH elevation rotation is defined as a clockwise rotation in the rotated elevation plane around the rotated $\mathbf{x}$-axis. The GH horizontal rotation is defined as counter-clockwise around the global $\mathbf{y}$-axis, with $0^{\circ} \mathrm{GH}$ horizontal rotation making the $\mathrm{GH}$ elevation rotation pure shoulder ab-/adduction, and $90^{\circ} \mathrm{GH}$ horizontal rotation making $\mathrm{GH}$ elevation rotation pure shoulder flexion/extension. The GH axial rotation is de- 
fined as being positive in clockwise direction (i.e. positive for internal/lateral rotation) around the upper arm vector (not common for right handed coordinate systems).

This decomposition is intuitively understood when performed by a person in a different order than the geometrical description, due to the singular nature of the $Y X Y$ rotation order (i.e. the unresolvable ambiguity between horizontal and axial rotation in some orientations). The intuitive decomposition is shown in Fig. 2.3 and described as follows:

1. First, a person elevates the arm from the zero-configuration (rotation from orientation (1) to (1) over angle $\gamma_{e}$ )

2. Secondly, the person performs horizontal rotation (rotation from orientation (1) to (2), over angle $\gamma_{h}$ ).

3. Finally, the person rotates axially (rotation from orientation (2) to (3), over angle $\gamma_{a}$.

Our initial- or zero-configuration is defined as the one with the upper arm pointing vertically down (orientation (1) in Fig. 2.3). In the zero-configuration the elbow is touching a person's side. For ease of reasoning, and to avoid ambiguity, we always assume $90^{\circ}$ elbow flexion.

\subsubsection{Kinematics of the Exoskeleton Shoulder}

For any exoskeleton shoulder we will use the same global axes as for the human shoulder (Sec. 2.2.1). Generically, a shoulder exoskeleton is regarded as a series of three sequential rotations. Although parallel exoskeleton shoulder actuation does exist [30], in this work we will compare several exoskeleton shoulder design configurations conceptually in Sec. 2.4.

The order of rotations in the human's zero configuration we will call the exoskeleton's rotation order. If the rotation order is for instance $X Y Z$, this implies that the first (most dominant) rotation axis is the global $\mathbf{x}$-axis, the second rotation axis is the global $\mathbf{y}$-axis and the third (least dominant) rotation axis is the global $\mathbf{z}$-axis. Note that these axes only coincide with the global axes in the human's zero orientation, and the second and third axes will be rotated away from the principal axes by moving the human arm.

Common rotation orders are the orthogonal designs (a bar denoting negative direction): $Y Z Y, \bar{X} Y Z, \bar{X} Z Y$ and $Y \bar{X} Z$. Also, several designs place the first rotation axis in none of the principal directions $[38,29,39]$ and do not necessarily 
Table 2.1: Joint zero-configurations of exoskeleton shoulder designs. ${ }^{*}$ Rotated $Z$, ${ }^{*}$ First Axis $<45^{\circ}$ rotated away from principal. A bar implies negative direction.

\begin{tabular}{ll}
\hline Order & Name[14, 15] \\
\hline$Y Z Y$ & ArmeoPower, ARMIN (chARMin, I-IV), Dampace, ExoRob, FRE- \\
& FLEX, IntelliArm, Limpact, (pneu-)WREX ${ }^{*}$, SUEFUL-7 \\
$\bar{X} Y Z$ & ABLE, BONES \\
$\bar{X} Z Y$ & L-Exos, CADEN-7 ${ }^{* *}$, RehabExos $^{* *}$, Salford ARE $^{(*)}$ \\
$Y \bar{X} Z$ & MEDARM $^{* *}$, MGA $^{* *}$, MULOS $^{*}$ \\
\hline
\end{tabular}

have the same kinematic properties as the four major orders. A list of several common designs is shown in Table 2.1. Useful references to these designs can be found in [14] and [15].

\subsection{METHOD}

\subsubsection{Shoulder ROM Projection}

We choose the Mollweide (or homolographic) projection [40] to expand a spherical surface onto a $2 \mathrm{D}$ plane. The Mollweide projection is preferred to an equirectangular projection (in which points of GH elevation and GH horizontal rotations form a rectangular grid), due to the equirectangular projection's ambiguity at the poles and the Mollweide projection's aesthetic properties.

We place the $90^{\circ} \mathrm{GH}$ elevation rotation and $90^{\circ} \mathrm{GH}$ horizontal rotation in the center of the map, as is shown by a gray human figurine in the background of the figure holding its arm in $\gamma_{h}=90^{\circ}, \gamma_{e}=90^{\circ}, \gamma_{a}=0^{\circ}$ orientation. This changes the map range from $0^{\circ}$ to $180^{\circ}$ in $\mathrm{GH}$ elevation rotation and $-90^{\circ}$ to $270^{\circ}$ in $\mathrm{GH}$ horizontal rotation, compared to conventional world maps in longitude and latitude. The $+270^{\circ}$ meridian is equivalent to the $-90^{\circ}$ meridian, and is therefore never used and shown as a dashed line. Equations to calculate the projection can be found in Appendix A.

In addition, we can also show the complete GH horizontal and elevation bounds by using a half-transparent shaded area, as is done in Fig. 2.1 and Fig. 2.5. This area is most of the time left out if it is irrelevant, and to limit the amount of information presented to the reader. 


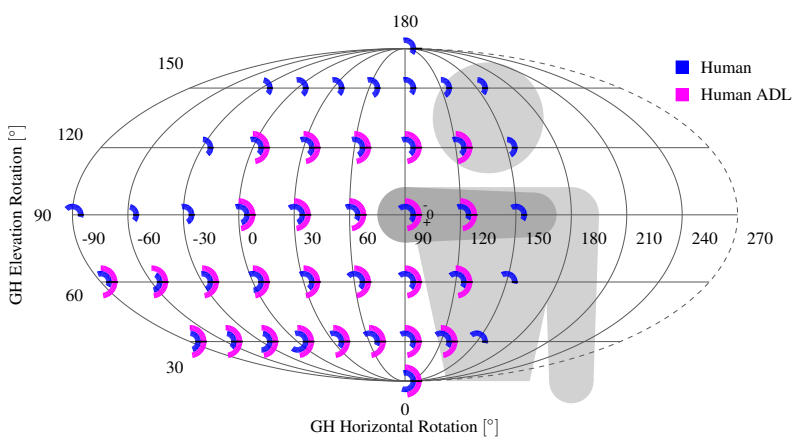

Figure 2.4: Measured ROM of the subject compared to specified ROM ADL-movements as measured in [24].

\subsubsection{Showing Axial Rotations}

The GH axial rotation ROM of the shoulder is shown on the Mollweide-grid on discrete locations. Discrete intervals are usable since the axial ROM varies rather smoothly over the complete sphere of GH horizontal and elevation rotation. The axial ROM is shown as an arc. Counter-clockwise rotation is negative axial rotation (i.e. external rotation), clockwise is positive axial rotation (i.e. internal rotation). Notice the small $0,-$ and + sign at the $90^{\circ}$ elevation pose of the figurine in the background in Fig. 2.1. In this work we space these arcs on intervals $30^{\circ}$ apart in horizontal and elevation rotation, although greater and smaller intervals are possible. If only a single axial orientation is shown (instead of a range), a thicker single line (like a clock hand) is shown, as is done in Fig. 2.1 for two example arm orientations.

\subsubsection{Measurement Method}

To measure the ROM of the shoulder we propose the following method to be performed for reachable chosen grid-points (a visual representation of the method is given in Fig. 2.3). This might not be an optimal way to determine the shoulder ROM, but we would like to emphasize that the visualization and not the measurement method is of main interest in this work. The method used to generate results in Sec. 2.4 is as follows: 


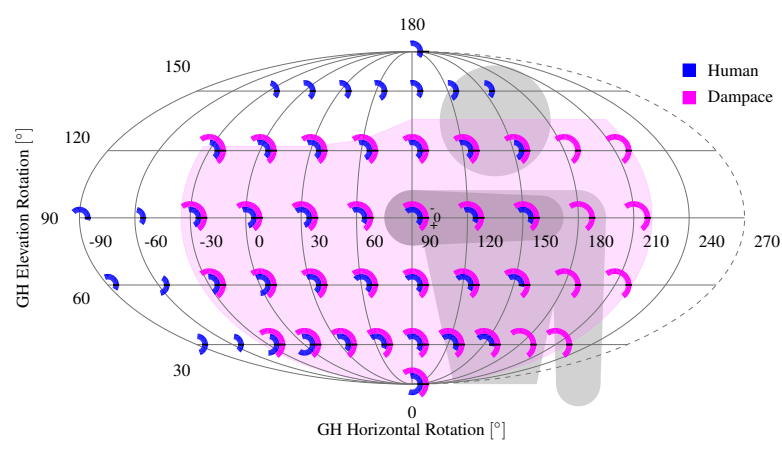

Figure 2.5: Real movement range of the Dampace [28], including joint limits, internal collisions and body collisions, compared to the human shoulder ROM.

1. The subject is placed in the initial-configuration $\left(\gamma_{h}=0^{\circ}, \gamma_{e}=0^{\circ}, \gamma_{a}=0^{\circ}\right)$ with the upper arm pointing down, and the forearm pointing forward $\left(90^{\circ}\right.$ elbow flexion).

2. The subject performs GH elevation rotation $\left(\gamma_{e}\right)$ of the arm, away from the zero-configuration.

3. The subject performs GH horizontal rotation $\left(\gamma_{h}\right)$. At the end of this motion, the forearm should be horizontal.

4. The subject performs GH axial rotation $\left(\gamma_{h}\right)$ to the extremes in both directions. These extremes give the complete range of axial rotation at this grid-point, and these have to be recorded.

The orientation can be recorded and confirmed through a (digital) goniometer, or through an (inertial) motion tracking system. If the subject cannot move his or her own arm, a physician has to perform the aforementioned steps.

To display properties of exoskeleton designs, the same protocol can be followed.

\subsection{EXAMPLE CASES}

The power of the method is demonstrated by using it for several examples of different design and presentation goals: 1) showing ROM measurement data of a subject, compared to an ADL measurement, 2) showing the limited ROM of an existing exoskeleton design when explained by classical movement ranges, 


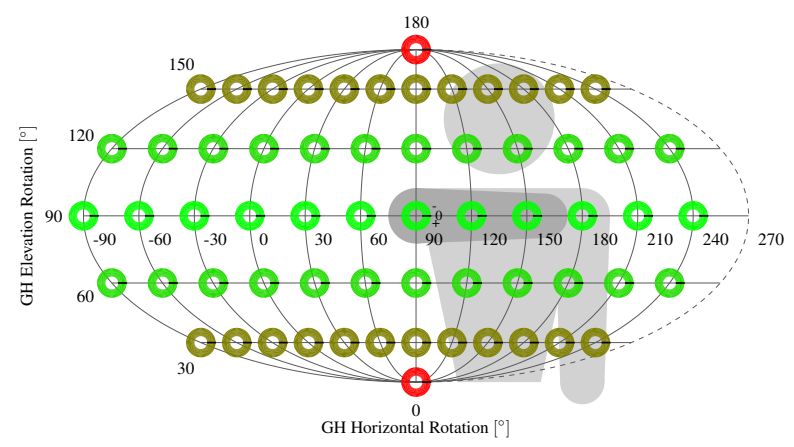

(a) $Y Z Y$

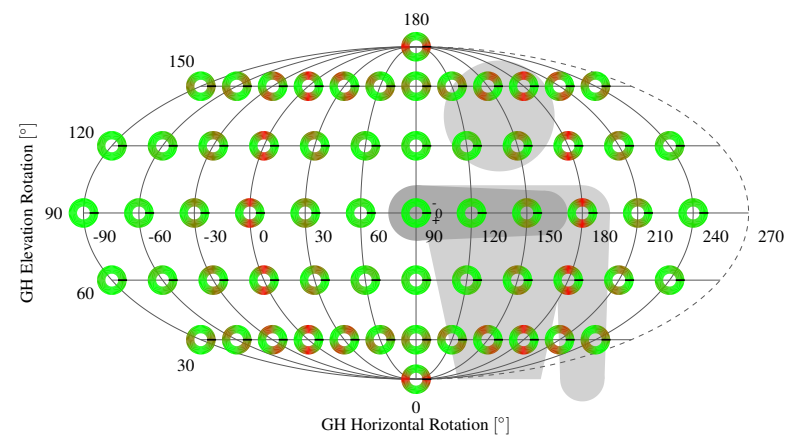

(b) $\bar{X} Y Z$

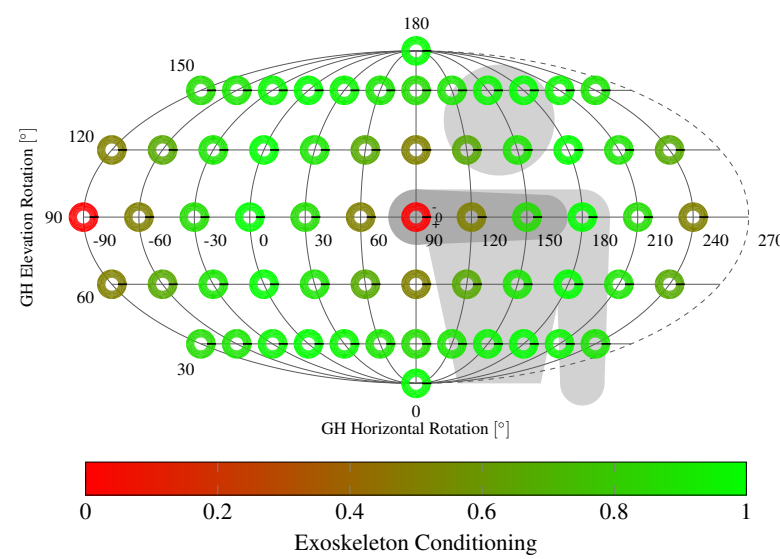

(c) $\bar{X} Z Y$

Figure 2.6: Illustration of conditioning of three common rotation orders: a) $Y Z Y$ b) $\bar{X} Y Z$ c) $\bar{X} Z Y$. Joint conditioning is shown as a red-to-green gradient (red = gimbal lock, green $=$ perfect conditioning) . 


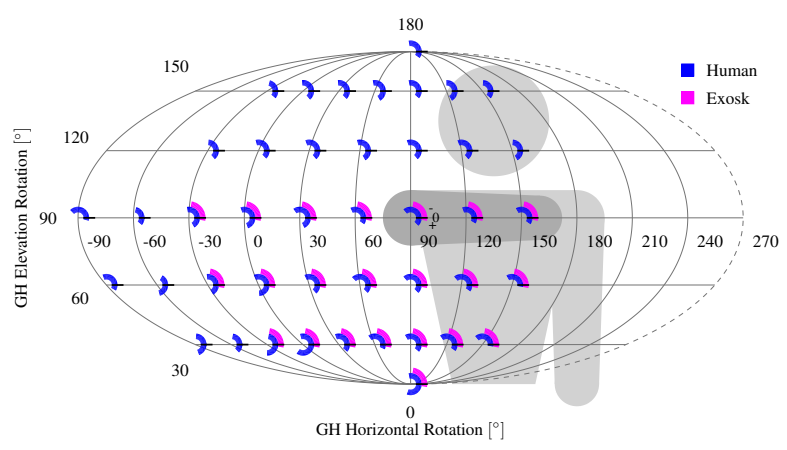

(a)

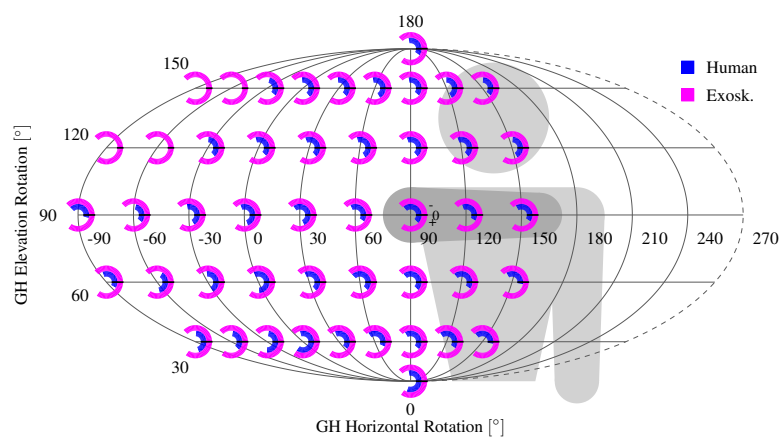

(b)

Figure 2.7: Implications of choosing mechanical joint limits in exoskeletons. a) All rotations of the exoskeleton limited to the minimum range b) All rotations of the exoskeleton limited to the maximum range. Both figures are only valid for $Y Z Y$ or $Y \bar{X} Y$.

3) showing kinematic exoskeleton design properties in terms of kinematic conditioning, 4) showing the complexity of the design problem of choosing mechanical joint limits for exoskeleton design.

The method was used to determine the human ROM (a healthy male subject, 19 y.o.), shown in Fig. 2.4. The subject was measured using a goniometer. Coupling is observed between the GH horizontal and GH elevation rotation and the range of GH axial rotation. The ROM is compared to the one measured in [24] for ADL movements to show that moving along the classical directions shows a different determined ROM without coupling effects. It cannot be ruled out that the different size of ROM is due to the subject(s) or the method. 
We show in Fig. 2.5 the measured ROM of the passive Dampace Exoskeleton [28], including the complete GH horizontal and elevation region in the shaded area. We compare the measured range with the human one, to show how well the exoskeleton supports the human ROM.

Next, we show the kinematic conditioning of three main exoskeleton shoulder types $Y Z Y, \bar{X} Y Z$ and $\bar{X} Z Y$ in Fig. 2.6. The exoskeleton conditioning is calculated by taking the absolute determinant of only the square rotation Jacobian of the system. The conditioning ranges therefore in value from 0 to 1 . The figures directly show the benefits of several rotation orders for a specified task ROM. It also shows that some rotation orders, such as $\bar{X} Z Y$ are better avoided. Therefore in [29] they actually placed the first axis under a $45^{\circ}$ angle, moving the singularity towards higher $\mathrm{GH}$ horizontal rotation angle.

Finally, we use the measurement data to show extreme choices of mechanically limiting rotations for $Y Z Y$ and $Y \bar{X} Y$ rotation orders (or for inverted versions of the rotation axes). If the minimal rotation ranges are picked, as in Fig. 2.7a, the design is very safe, but very conservative and unusable. If the maximal rotation ranges are picked, as in Fig. 2.7b, the human achieves full range of motion, but the exoskeleton cannot be inherently mechanically safe and needs angle limitations in software.

\subsection{Discussion}

The visualization method can be used to display a large variety of useful information about standard human shoulder ROM, pathological ROM, ADL ROM, assistive device ROM and mechanical, kinematic or dynamic device properties.

Compared to presentation of classical movement ranges of the human shoulder in tables, this method does take more publication space, but gives a more complete picture. The addition of our method to the globe system[37] is the possibility to show complete ROM directly, including axial rotation, without using different camera viewpoints. Compared to plotting 3D points or a surface in a 2D image, our method is clear and less ambiguous, also directly including GH axial rotations.

Using our method to display device ROM is useful if its ROM is compared to the human ROM and show its dynamic or kinematic properties over the $h u$ man workspace. For a separate robotic device the joint limits could already be sufficient to determine their own complete ROM. 
The given examples demonstrate that for a select set of challenges, the extended effort needed to acquire the full set of measurements needed for the visualization are worth the effort in collection.

A minor drawback, due to the ISB decomposition of rotation into three Euler angles, is the representation singularity in the arm's zero-configuration. A shoulder flexion to hyper-extension motion with the forearm forward would imply an instantaneous $180^{\circ}$ jump in GH horizontal (from $+90^{\circ}$ to $-90^{\circ}$ ) and $\mathrm{GH}$ axial rotation (from $+90^{\circ}$ to $-90^{\circ}$ ). This jump in angles is counter-intuitive.

Future work will include simplifying the required data collection, using inertial measurement systems. Measuring more subjects will help introducing a statistical certainty measure of the achieved ROM, which can be included in the visualization. Finally, this method will be evaluated with clinicians, industrial partners to show its usefulness in displaying progression of shoulder rehabilitation and the benefit of assistive and rehabilitation devices. 
DIFFERENTIAL INVERSE KINEMATICS OF A REDUNDANT 4R EXOSKELETON SHOULDER JOINT

Arvid Q.L. Keemink, Gijs van Oort, Martijn Wessels and Arno H.A. Stienen

The Singularity is Near.

— Raymond Kurzweil, 2006

Abstract-Most active upper-extremity rehabilitation exoskeleton designs incorporate a $3 R$ rotational shoulder joint with orthogonal axes. This kind of joint has poor conditioning close to singular configurations when all joint axes become coplanar, which reduces its effective range of motion. We investigate an alternative approach of using a redundant non-orthogonal $4 R$ rotational shoulder joint. By inspecting the behavior of the possible nullspace motions, a new method is devised to resolve the redundancy in the differential inverse kinematics (IK) problem. A 1D nullspace global attraction method is used, instead of naive nullspace projection, to guarantee proper convergence. The design of the exoskeleton and the proposed IK method ensure good conditioning, avoid collisions with the human head, arm and trunk, can reach the entire human workspace, and outperforms conventional $3 R$ orthogonal exoskeleton designs in terms of lower joint velocities and by avoiding body collisions.

LIST OF USED SYMBOLS AND ABBREVIATIONS

\begin{tabular}{ll}
\hline Symbol & Explanation \\
\hline$\emptyset$ & the empty set \\
$\mathcal{A}_{n s}(\boldsymbol{\Gamma}, \mathbf{q})$ & subset of $\mathcal{C}_{n s}$ reachable from current joint configuration \\
$\mathcal{C}_{n s}(\boldsymbol{\Gamma})$ & self-motion-manifold of exoskeleton or arm configuration \\
$\mathcal{D}_{n s}(\boldsymbol{\Gamma}, \mathbf{q})$ & subset of $\mathcal{C}_{n s}$ disjointed from current joint configuration \\
$e_{\mathcal{Q}}$ & scalar feedback error along $\mathcal{Q}_{n s}$
\end{tabular}

This chapter is under review in the IEEE/RAS-EMBS Transactions on Neural Systems \& Rehabilitation Engineering (TNSRE). 
$f(\mathbf{q}) \quad$ scalar potential parameterized by joint angles $\mathbf{q}$

$\mathcal{F}(\mathbf{q}) \quad$ generic form of a forward kinematic function

li super-/subscript: relating to the global reference frame subscript: relating to exoskeleton links geometrical Jacobian

discrete-time time instant

ns feedback gain subscript: related to the nullspace motions the nullspace of the matrix argument nullspace projector matrix vector of joint angles a valid configuration in the next self-motion-manifold joint-space attractor the minimal point, closest to the attractor differential inverse kinematics minimum-norm solution initial configuration of the self-motion-manifold subset of $\mathcal{C}_{n s}$ connected to current joint configuration

link radius

$\mathbf{R} \quad$ three-dimensional rotation matrix

$\mathbb{R} \quad$ the set of all real numbers

$S O(3) \quad$ group of all $3 \mathrm{D}$ rotations about the origin $t$ time

T discrete sample time

u unit-vector

$\mathbf{u}_{\mathcal{N}} \quad$ basis of the nullspace of the Jacobian

ua subscript: relating to the human's upper arm

$\mathbf{U}, \mathbf{V} \quad$ left and right eigenvector matrices as result from SVD

w any dummy vector in $\mathbb{R}^{4}$

$\mathcal{W}_{h} \quad$ human shoulder workspace

$\mathbf{x}, \mathbf{y}, \mathbf{z} \quad$ principal axes vectors

$x, y, z \quad$ subscript: directions

$X, Y, Z \quad$ positive directions along principal axis vectors

$\mathbb{Z} \quad$ set of all the integer numbers 


\begin{tabular}{ll}
$\alpha$ & swept angle of a collision cone \\
$\beta$ & scalar used to make tanh $(\beta x)$ approach $\operatorname{sgn}(x)$ \\
$\gamma_{a}$ & axial glenohumeral rotation angle \\
$\gamma_{e}$ & glenohumeral elevation angle \\
$\gamma_{h}$ & horizontal glenohumeral rotation angle \\
$\Gamma$ & vector of shoulder angles \\
$\delta(\xi)$ & scalar distance along $\mathcal{C}_{n s}$ \\
$\Delta x$ & spatial numerical integration step \\
$\eta$ & conditioning threshold \\
$\theta$ & relative rotation of the first axis of rotation \\
$\boldsymbol{\Theta}$ & vector of relative rotations of the first axis of rotation \\
$\lambda$ & eigenvalue \\
$\rho$ & nullspace vector scalar \\
$\sigma$ & singular value \\
$\Sigma$ & matrix of singular values as result from SVD \\
$\phi$ & exoskeleton arc \\
$\Phi$ & vector of exoskeleton arcs \\
$\xi$ & joint-space integration variable \\
$\omega$ & vector of axis of rotation of the exoskeleton joints \\
$\Omega$ & rotational velocity vector of the shoulder \\
\hline &
\end{tabular}

\begin{tabular}{ll}
\hline Abbrev. & Explanation \\
\hline$n \mathrm{R}$ & $n$ degrees of freedom of rotation \\
CoR & center of rotation \\
DOF & degree of freedom \\
IK & inverse kinematics \\
SVD & singular value decomposition \\
\hline
\end{tabular}

\subsection{INTRODUCTION}

Upper-Extremity exoskeletons are commonly used for rehabilitation purposes, tele-operation and task training $[39,41,14,15]$. One major issue with these 


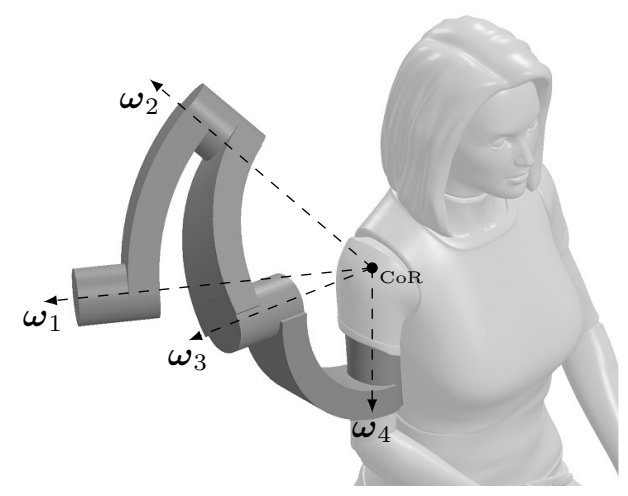

Figure 3.1: Schematic overview of the proposed $4 \mathrm{R}$ shoulder joint, showing a person using the exoskeleton joint to move the upper-arm. The joint is comprised of three concentric arcs with decreasing radii. The dashed arrows indicate the four rotation axes of the joint that intersect in the Center of Rotation (CoR). Note that the fourth joint axis is inside the human arm. Translations of the shoulder complex will be taken care of by an external translation mechanism [43] attached to the first motor (not shown).

conventional exoskeletons is that they cannot match the full human shoulder workspace due to conditioning and collision problems.

The shoulder of upper-extremity rehabilitation exoskeletons usually comprises three sequential rotational (3R) degrees of freedom (DOFs) [42, 39, 43]. Such a system's kinematics are fully described by three implicit Euler angles. Any system with sequential rotations has singular configurations, also known as gimbal lock, when rotation axes become collinear or coplanar. During gimbal lock in a shoulder exoskeleton joint the user loses one rotational direction to move in [44]. Near the singular configuration an inverse kinematics algorithm has to handle the poor conditioning, which results in high motor velocities and high experienced inertia.

We propose to add an extra rotational DOF to the exoskeleton joint, and to make all four rotation axes non-orthogonal in order to 1) avoid singular configurations, 2) make it easier to avoid collisions with the human body and 3) achieve a range of motion larger than the one of the human shoulder. An example design is shown in Fig. 3.1. The $4 \mathrm{R}$ shoulder joint is to be used in $3 \mathrm{R}$ admittance control mode with bounded velocities and accelerations, similar to [39, 45, 46]. Therefore, we need an inverse kinematics (IK) algorithm to pick a good set of four joint angles, or their velocities, that imposes the right orientation on the human shoulder without precomputed trajectories. 
A $4 \mathrm{R}$ shoulder joint for exoskeletons has been implemented twice recently. In $[47,48,49]$, a one-to-one configuration mapping with unexplained interpolation on 89 upper-arm configurations is used. A non-orthogonal exoskeleton configuration was found through a genetic algorithm. Although their method appears to work, it does not give insight into the problem, and has no a-priori guarantee or explanation if it will always work for any design. In [50], the focus is on a passive orthogonal $4 \mathrm{R}$ exoskeleton for macaques. Their analysis focuses on the theoretical feasibility of the $4 \mathrm{R}$ principle, to determine if avoiding joint limits and poor conditioning is possible, and do not focus on any IK method. They incorporate body collisions implicitly in the joint limits. They conclude for their fully orthogonal design that it should be possible to always stay away from gimbal lock and joint limit collisions.

The goal of this work is to detail the differential IK algorithm for this redundant $4 \mathrm{R}$ exoskeleton shoulder joint. We focus solely on the rotation of the human shoulder, with translations of the joint $[51,52,53,39,43]$ taken care of by a translation mechanism [43]. Furthermore, the fourth rotation axis is initially chosen to be parallel to the long axis of the upper arm, such that the problem reduces in complexity to a $3 R$-to- $2 R$ mapping instead of a $4 R$-to-3R mapping.

\subsubsection{Background}

Avoiding gimbal lock for a $4 \mathrm{R}$ gimbal system was achieved by NASA for flightsimulators $[54,55,56]$. A method was developed to steer the fourth, least dominant, DOF to attempt to improve the gimbal conditioning. Their method works in a collision-free setting, valid for flight-simulators, but will not work when the human body restricts the solution space. Other $4 \mathrm{R}$ systems have been proposed to rotate cameras [57]. They take advantage of nullspace motions and DOF weighting in the Jacobian pseudo-inverse (see Sec. 3.2.5). This method too is limited to collision-free settings. A general robotics redundancy resolution is proposed by Siciliano et al. [58], by applying gradient-nullspace-projection (see Sec. 3.4) of a (cost) minimization objective in jointspace. This commonly results in achieving only local optima. The structure of the nullspace and constraints is not analysed, making the resulting behavior sub-optimal and lacking any guarantees about conforming to constraints.

We expand on this previous work and propose a more generic method of analysis and solution to the problem for non-orthogonal $4 \mathrm{R}$ shoulders that explicitly takes into account collision with the body and the human arm. We overcome 
some drawbacks of naive gradient projection, while ensuring quasi-static convergence to a well-defined nullspace-configuration 'minimum'. Although we will discuss one specific design in this work, the analysis and IK method is applicable to other $4 \mathrm{R}$ designs.

\subsection{KINEMATICS}

We use 'shoulder' to refer to the human 3R shoulder joint and 'exoskeleton' to refer to the new $4 \mathrm{R}$ exoskeleton joint. In the subsequent text, vectors and matrices are denoted in boldface, and unit vectors are denoted by $\mathbf{u}$. The only exceptions are coordinate-frame axes which are given by $\mathbf{x}, \mathbf{y}$ or $\mathbf{z}$. A 3-by-3 rotation matrix around a principal axis is specified by $\mathbf{R} \in S O(3)$, with subscript $x, y$ or $z$.

\subsubsection{Axes Definitions}

For the shoulder and exoskeleton we use definitions of axes and angles from the ISB [25], as shown in Fig. 3.2a and Fig. 3.2b. An inertial global frame is attached to the glenoid in its Center of Rotation (CoR) of the shoulder. Any vector expressed in this global frame will have left superscript $g$. For the right arm, the global $\mathbf{x}$-axis points in the dorsoventral direction (forward), the global $\mathbf{y}$-axis points in the anteroposterior direction (upward) and the global $\mathbf{z}$-axis points into the mediolateral direction (outward to the right).

\subsubsection{Kinematics of the Shoulder}

The human shoulder orientation can be decomposed around these three axes into YXY intrinsic Euler angle rotations (stated in the order of most-middleleast dominant, i.e. a more dominant rotation also rotates less dominant axes) relative to the global frame. The angle decomposition is shown in Fig. 3.2c. The zero-orientation is defined where the upper arm is pointing vertically down, with the elbow touching a person's side (see Fig. 3.2c arm pose (0)). Geometrically the zero-orientation would give the upper-arm a unit vector in negative global y, i.e. ${ }^{g} \mathbf{u}_{u a}=-{ }^{g} \mathbf{u}_{y}$. The forearm would then point from the elbow forward, parallel to ${ }^{g} \mathbf{u}_{x}$.

The human elbow can reach a subset of points on the surface of a sphere, defined by horizontal rotation $\gamma_{h}$ and elevation rotation $\gamma_{e}$. The third axial upperarm rotation, internal and external rotation, is described directly by $\gamma_{a}$. These 


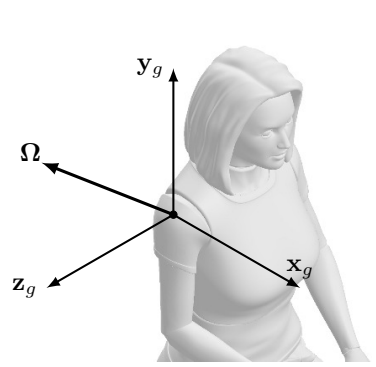

(a)

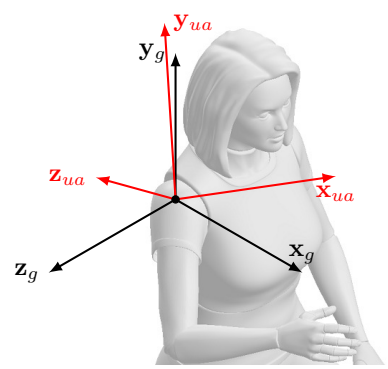

(b)
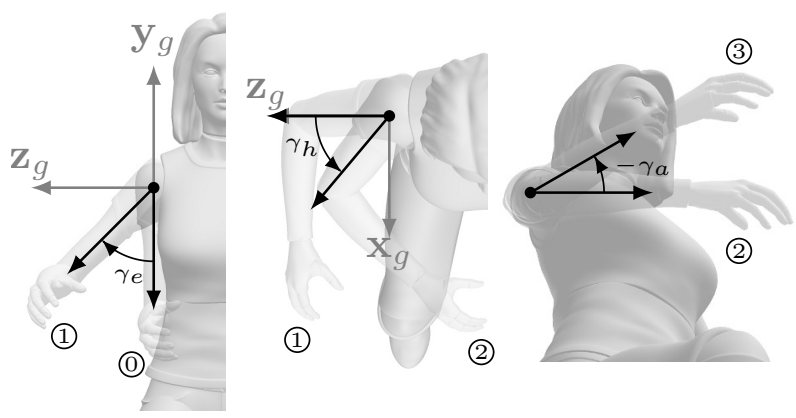

(c)

Figure 3.2: a) ISB axes definition of the global frame $\left(\mathbf{x}_{g}, \mathbf{y}_{g}, \mathbf{z}_{g}\right)$, showing also the rigid body instantaneous rotational velocity vector of the shoulder $\boldsymbol{\Omega}$ that will bring the upper arm to the configuration in b). b) Showing the rotated upper arm (ua) frame (red) at the end of the rotation. c) Interpretation of the ISB angle definitions $\left(\gamma_{h}, \gamma_{e}, \gamma_{a}\right)$ for decomposing the arm configuration in b). It is more insightful to elevate first (from pose (1) to (1), over angle $\gamma_{e}$ ), then rotate horizontally (from pose (1) to (2), over angle $\gamma_{h}$ ) and then rotate axially (from pose (2) to (3), over angle $\gamma_{a}$ ), as shown from left to right in the figure. In the actual ISB definitions, the arm orientation has to be decomposed (most to least dominant rotation) into plane-of-elevation rotation (around $\mathbf{y}_{g}$, with angle $\gamma_{h}$ ), elevation rotation (around a rotated local $X$, with angle $\gamma_{e}$ ) and axial rotation (around double rotated local $Y$, with angle $\gamma_{a}$ ). The mathematical description is given in Sec. 3.2.2. 
angles are combined in $\Gamma=\left[\gamma_{h}, \gamma_{e}, \gamma_{a}\right]^{T}$. Positive elevation is defined as a clockwise rotation around rotated $X$. The forward kinematics of the shoulder coordinate frame are hence described by a chain of three rotation matrices

$$
{ }^{g} \mathbf{R}_{u a}(\boldsymbol{\Gamma})=\mathbf{R}_{y}\left(\gamma_{h}\right) \mathbf{R}_{x}\left(-\gamma_{e}\right) \mathbf{R}_{y}\left(-\gamma_{a}\right)
$$

\subsubsection{Exoskeleton Geometry}

The exoskeleton comprises four revolute joints (the exoskeleton axes). The orientation of the first rotation axis is rotated from ${ }^{g} \mathbf{y}$ over two sequential $Y X$ rotations $\boldsymbol{\Theta}=\left[\theta_{1}, \theta_{2}\right]^{T}$, as shown in Fig. 3.3a. The four exoskeleton axes are separated by three $\operatorname{arcs} \boldsymbol{\Phi}=\left[\phi_{1}, \phi_{2}, \phi_{3}\right]^{T}$, as shown in Fig. 3.3b. These arcs are commonly $90^{\circ}$ in $3 \mathrm{R}$ designs, but we choose a different design. The rotational motor degrees of freedom are described by four rotation angles $\mathbf{q}=$ $\left[q_{1}, q_{2}, q_{3}, q_{4}\right]^{T}$. The zero-configuration of the exoskeleton is as it is spread out in the singular plane (the $Y Z$ plane rotated over angles $\Theta$ ) in Fig. 3.3b.

We found that for any $4 \mathrm{R}$ shoulder exoskeleton the joint axes do not have to be perpendicular to be able to reach the complete range of all possible 3D rotations in $S O(3)$. To be able to generate all elements in $S O(3)$ at least once, the necessary and sufficient condition ( $\boldsymbol{\Phi}$ in radians) is

$$
\left\|\boldsymbol{\Phi}-\frac{\pi}{2}\right\|_{1}=\sum_{i=1}^{3}\left|\phi_{i}-\frac{\pi}{2}\right| \leq \frac{\pi}{2} .
$$

Eq. (3.2) puts limits on valid combinations of $\operatorname{arcs} \boldsymbol{\Phi}$.

For the conceptual design in Fig. 3.1 the design parameters are given in Table 3.1. These design parameters were derived from analysing the exoskeleton with the method outlined in this chapter to find a proper combination of design parameters. The sizes correspond to a developed 1:2 scale model of a $4 \mathrm{R}$ exoskeleton, and they do not necessarily constitute proper mechanical design optimized for stiffness and size. From Table 3.1 it can be seen that $\|\boldsymbol{\Phi}-\pi / 2\|_{1}=$ $5 \pi / 12$, conforming to (3.2). 
Table 3.1: Design Parameters of the System

\begin{tabular}{lll}
\hline Symbol & Value & Explanation \\
\hline$\theta_{1}[\mathrm{deg}]$ & -35 & First axis angle Y \\
$\theta_{2}[\mathrm{deg}]$ & +90 & Second axis angle X \\
$\phi_{1}[\mathrm{deg}]$ & 45 & First link arc \\
$\phi_{2}[\mathrm{deg}]$ & 60 & Second link arc \\
$\phi_{3}[\mathrm{deg}]$ & 90 & Third link arc \\
$\eta[-]$ & 0.25 & Conditioning threshold \\
$r_{1}[\mathrm{~m}]$ & 0.288 & First link radius \\
$r_{2}[\mathrm{~m}]$ & 0.222 & Second link radius \\
$r_{3}[\mathrm{~m}]$ & 0.156 & Third link radius \\
$r_{m}[\mathrm{~m}]$ & 0.037 & Motor radius \\
$r_{a}[\mathrm{~m}]$ & 0.06 & Arm radius \\
$\rho[\mathrm{rad} / \mathrm{s}]$ & 1 & NS integration velocity \\
$\Delta T[\mathrm{~s}]$ & 0.001 & Discrete sample time \\
$\Delta x[\mathrm{rad}]$ & 0.0001 & Spatial integration step \\
\hline
\end{tabular}

\subsubsection{Forward Kinematics of the Exoskeleton}

The exoskeleton configuration ( $u a$ with respect to $g$ ) is a chain product of

$$
{ }^{g} \mathbf{R}_{u a}(\mathbf{q}, \boldsymbol{\Theta}, \boldsymbol{\Phi})={ }^{g} \mathbf{R}_{1}(\boldsymbol{\Theta}){ }^{1} \mathbf{R}_{4}(\mathbf{q}, \boldsymbol{\Phi}){ }^{4} \mathbf{R}_{u a}\left(q_{4}\right),
$$

with ${ }^{g} \mathbf{R}_{1}(\boldsymbol{\Theta})$ the initial rotation over angles $\boldsymbol{\Theta},{ }^{1} \mathbf{R}_{4}(\mathbf{q}, \boldsymbol{\Phi})$ the rotations over angles q, separated by arcs $\boldsymbol{\Phi}$ (see Fig. 3.3b.) and ${ }^{4} \mathbf{R}_{u a}\left(q_{4}\right)$ the final axial rotation of the upper arm. This gives the forward kinematics of the $4 \mathrm{R}$ exoskeleton joint. Note that only $\mathbf{q}$ is controlled and $\boldsymbol{\Theta}$ and $\boldsymbol{\Phi}$ are design parameters.

The mechanical coupling of the shoulder and exoskeleton results in

$$
{ }^{g} \mathbf{R}_{u a}(\boldsymbol{\Gamma})={ }^{g} \mathbf{R}_{u a}(\mathbf{q}, \boldsymbol{\Theta}, \mathbf{\Phi}),
$$

which forms the basis of the inverse kinematics problem. 


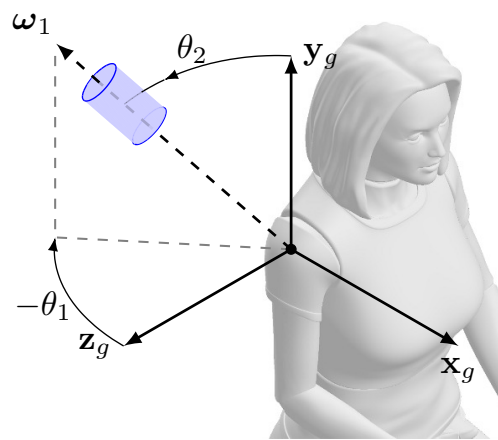

(a)

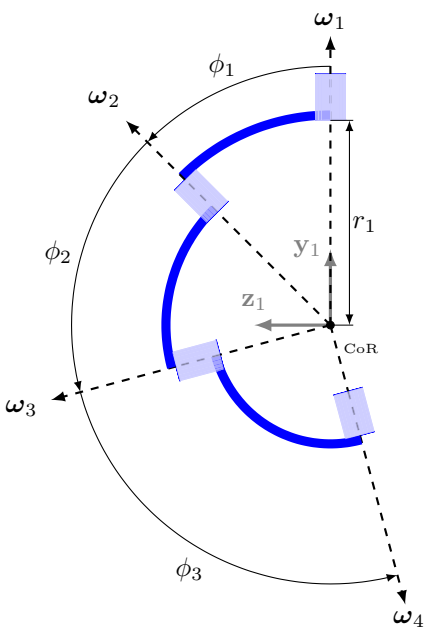

(b)

Figure 3.3: a) Definition of $\Theta$ angles for rotating the first rotation axis $\omega_{1}$ relative to global ${ }^{g} \mathbf{y}$. Only the first motor is shown for clarity. b) Definition of arcs (link 'lengths') between two consecutive rotation axes given by $\phi_{1}, \phi_{2}$ and $\phi_{3}$. The exoskeleton is placed in a singular $\mathbf{Y Z}$ plane in the 1 -frame. By definition, each rotation axis rotates around its local $Y$ and is separated by an $\operatorname{arc} \phi_{j}$, rotated around local $X$. The distance of the first motor w.r.t. the CoR is given by $r_{1}$. The human body is not shown for clarity.

\subsubsection{Inverse Kinematics of the Exoskeleton}

Solving the inverse kinematics (IK) problem means finding $\mathbf{q}$ or its time derivative from the known $\boldsymbol{\Theta}, \boldsymbol{\Phi}$ and shoulder angles $\Gamma$, shoulder rotational velocity $\boldsymbol{\Omega}$, and acceleration $\boldsymbol{\Omega}$ (see Fig. 3.2a and Fig. 3.2b).

Solving the IK in (3.4) analytically is in general impossible and requires iterative approaches or numerical integration. Another problem is that the exoskeleton is composed of 4-DOF, while the shoulder is uniquely described by 3-DOF (when not in a representation singularity). Hence, there is no unique inverse mapping.

First order differential forward kinematics are given by

$$
{ }^{g} \boldsymbol{\Omega}=\left({ }^{g} \dot{\mathbf{R}}_{u a}{ }^{g} \mathbf{R}_{u a}^{T}\right)^{\vee}=\mathbf{J}(\mathbf{q}) \dot{\mathbf{q}},
$$


with $(\cdot)^{\vee}$ the inverse skew-operator. Geometrically, Jacobian $\mathbf{J}(\mathbf{q})$ is comprised of the four joint axes, expressed in the global frame:

$$
\begin{aligned}
\mathbf{J}(\mathbf{q})= & {\left[{ }^{g} \boldsymbol{\omega}_{1},{ }^{g} \boldsymbol{\omega}_{2},{ }^{g} \boldsymbol{\omega}_{3},{ }^{g} \boldsymbol{\omega}_{4}\right] } \\
= & {\left[{ }^{g} \mathbf{R}_{1}(\boldsymbol{\Theta}){ }^{1} \mathbf{u}_{y},{ }^{g} \mathbf{R}_{2}\left(\boldsymbol{\Theta}, \phi_{1}, q_{1}\right)^{2} \mathbf{u}_{y},\right.} \\
& \left.{ }^{g} \mathbf{R}_{3}\left(\boldsymbol{\Theta}, \phi_{1}, \phi_{2}, q_{1}, q_{2}\right)^{3} \mathbf{u}_{y},{ }^{g} \mathbf{R}_{4}\left(\boldsymbol{\Theta}, \boldsymbol{\Phi}, q_{1}, q_{2}, q_{3}\right)^{4} \mathbf{u}_{y}\right]
\end{aligned}
$$

The Jacobian is not square and not directly invertible. A candidate inverse is the one that minimizes the squared Euclidian-norm $\left(L_{2}\right)$ of the joint velocities. This gives the minimum norm solution $\dot{\mathbf{q}}_{m n}$ :

$$
\begin{aligned}
\dot{\mathbf{q}}_{m n} & =\mathbf{J}^{T}(\mathbf{q})\left(\mathbf{J}(\mathbf{q}) \mathbf{J}^{T}(\mathbf{q})\right)^{-1} g \boldsymbol{\Omega} \\
& =\mathbf{J}^{\dagger}(\mathbf{q})^{g} \mathbf{\Omega} .
\end{aligned}
$$

The $\mathbf{J}^{\dagger}(\mathbf{q})$ is a right pseudo-inverse of $\mathbf{J}(\mathbf{q})$.

This minimum norm solution gives joint velocities that might still make the exoskeleton singular or collide with the human body, and is therefore not sufficient to operate the exoskeleton.

A more general solution to the differential inverse kinematics is of the form

$$
\dot{\mathbf{q}}_{i k}=\dot{\mathbf{q}}_{m n}+\dot{\mathbf{q}}_{n s},
$$

in which $\dot{\mathbf{q}}_{n s}$ is a nullspace joint velocity [58] that is used to steer the exoskeleton away from poor conditioning and body collisions, as will be explained in detail in Sec. 3.3.2.

\subsection{PRELIMINARIES}

In this section we investigate the constraints and assumptions for our method. An in depth analysis of the 'nullspace motions' is presented in Sec. 3.3.2-4.

\subsubsection{Jointspace Constraints}

During operation of the exoskeleton several events are not allowed to happen, which are expressed as jointspace constraints. These constraints will be discussed in order of decreasing importance. Methods to calculate the constraints can be found in the supplementary material. 


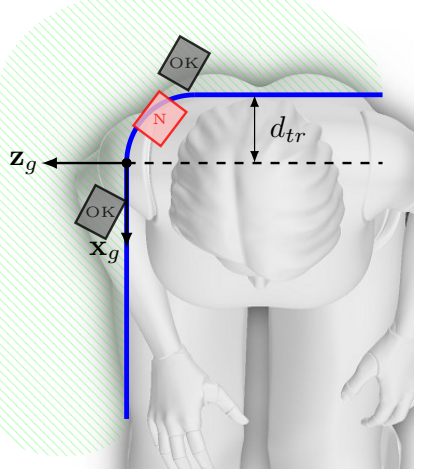

(a)

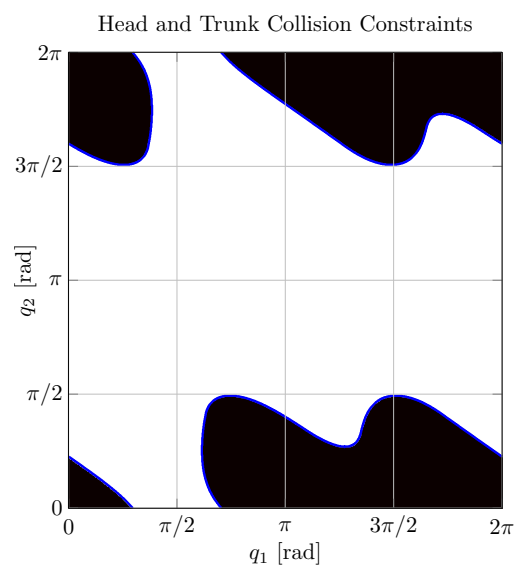

(b)

Figure 3.4: a) Top view of a person showing the relevant boundary surface that prevents collision with the human head and trunk, in blue. The shaded green area gives the allowed space for motors 1, 2 and 3. Two examples of correct motor locations are shown as gray squares ('OK'). A single example of a not allowed location is given by the red square (letter ' $N$ '). b) Stationary body collision conditions are shown, projected onto the $q_{1}-q_{2}$-plane. The dark background regions imply collision with the human trunk. Allowed angles for the first two DOFs are constrained to the white area. Note that the space repeats every $2 \pi \mathrm{rad}$.

The links and motors should not collide with the stationary human head and trunk

This condition can be simplified by letting none of the moving motors (except for the one of $\omega_{4}$ ) cross some virtual planes close to the body. A first constraint plane is the global $X Y$-plane. A second constraint plane is the global $Y Z$-plane, but moved over a distance $d_{t r}$ (the trunk thickness, assumed to be $0.1 \mathrm{~m}$ ) to the back of the human. The sharp corner over the shoulder is unnecessarily restrictive, therefore a smooth quarter circle with radius $d_{t r}$ connects both planes. The allowed region is the union of both regions on the 'outside' of these planes, as is illustrated in Fig. 3.4a.

The stationary head-trunk collisions are only dependent on the orientation of $\omega_{2}$ and $\omega_{3}$, the motor size, and their distance from the CoR. Therefore kinematically, the constraints are only dependent on $\theta_{1}, \theta_{2}, \phi_{1}, \phi_{2}, q_{1}$ and $q_{2}$. The constraints are visualized in the $q_{1}-q_{2}$-plane in Fig. 3.4b. 


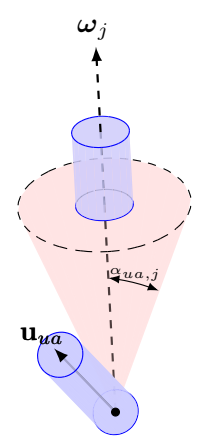

(a)

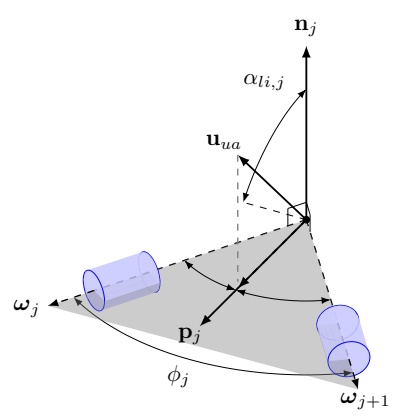

(b)

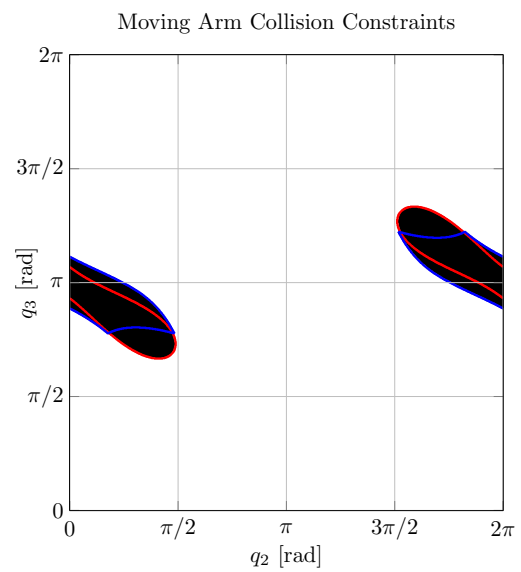

(c)

Figure 3.5: a) The arm (approximated by a cylinder) needs its unit vector $\mathbf{u}_{u a}$ to stay outside of a virtual cone (half angle $\alpha_{u a, j}$ ) around the joint axis to avoid collision with motors. b) In order to avoid collision with the link, the arm cannot be close to the plane formed between two joint axes. The unit vector $\mathbf{u}_{u a}$ cannot have an angle from $\mathbf{n}_{j}$ greater than $\alpha_{l i, j}$. The shown configuration is valid. c) Moving arm collision conditions are projected on the $q_{2}-q_{3}$-plane. The dark background regions imply collision with the human arm. The red line and blue line encompass the motor and link collision regions respectively. Note that the space repeats every $2 \pi \mathrm{rad}$.

We have chosen $\boldsymbol{\Theta}$ and $\boldsymbol{\Phi}$ together with the link arc-radii such that the amount of head-trunk collision configurations is minimized.

The links and motors should not collide with the moving upper arm

This can be achieved by constraining upper arm (approximated as a cylinder with radius $r_{u a}=0.07 \mathrm{~m}$ ), to have its unit vector $\mathbf{u}_{u a}$ outside a cone (half angle $\alpha_{u a, j}$ ) around an exoskeleton axis $\omega_{j}$. This idea is illustrated in Fig. 3.5a.

The upper arm should also never hit the links between the motors. It could only hit link $1\left(\operatorname{arc} \phi_{1}\right)$ and link $2\left(\operatorname{arc} \phi_{2}\right)$ (although this link can be avoided by proper choice of $\phi_{2}$ and $\phi_{3}$ ) both with thickness $d_{l i}$. If the previous constraint is violated, this is even more problematic if the arm is close enough to the plane formed by the rotation axes to actually hit the link. This idea is illus- 
trated in Fig. 3.5b. The constraints are visualized in the $q_{2}-q_{3}$-plane in Fig. 3.5c. Our choice of values for $\boldsymbol{\Phi}$ ensures we can fully avoid arm collision with link 2 .

The motors should not collide with other motors

By designing the link arcs and radii properly, this problem can be fully avoided. Hence this is not further discussed.

The exoskeleton should not approach singularity

The exoskeleton is only fully singular (rank 2) if all joint axes lie in a common plane. In that case, all possible sub-determinants of $\mathbf{J}(\mathbf{q})$ are 0 and the shoulder has only two rotational directions left to move in. This configuration should be avoided at all times. This can be achieved by not approaching singularity. Therefore, we need at least some combination of three out of all four exoskeleton axes to have 'sufficient conditioning', i.e. to not be coplanar. The worst case maximal value of all sub-determinants should be higher than some chosen lower bound.

The conditioning of the exoskeleton joint is shown in the $q_{2}-q_{3}$-plane in Fig. 3.6. The method allows for any combination of three axes to become singular (and swap sign of the determinant), as long as another combination of three axes is not. Since $q_{4}$ is not involved in the Jacobian, this condition depends on $q_{2}, q_{3}$ and $\boldsymbol{\Phi}$, but not on $\boldsymbol{\Theta}$.

\subsubsection{Nullspace Motions and Configurations}

The redundancy of the $4 \mathrm{R}$ exoskeleton allows its axes to rotate 'internally' without changing the shoulder rotation $\Gamma$. The nullspace of $\mathbf{J}(\mathbf{q})$ has a dimensionality of 1 . This means that for any arm configuration there is a one-dimensional manifold consisting of an infinite number of joint angle combinations possible in the exoskeleton that do not change the orientation of the human arm.

To use these internal motions to our benefit we aim to find the complete nullspace of $\mathbf{J}(\mathbf{q})$ :

$$
\mathcal{N}(\mathbf{J}(\mathbf{q}))=\left\{\dot{\mathbf{q}}_{n s} \in \mathbb{R}^{4}: \mathbf{J}(\mathbf{q}) \dot{\mathbf{q}}_{n s}=\mathbf{0}\right\} .
$$




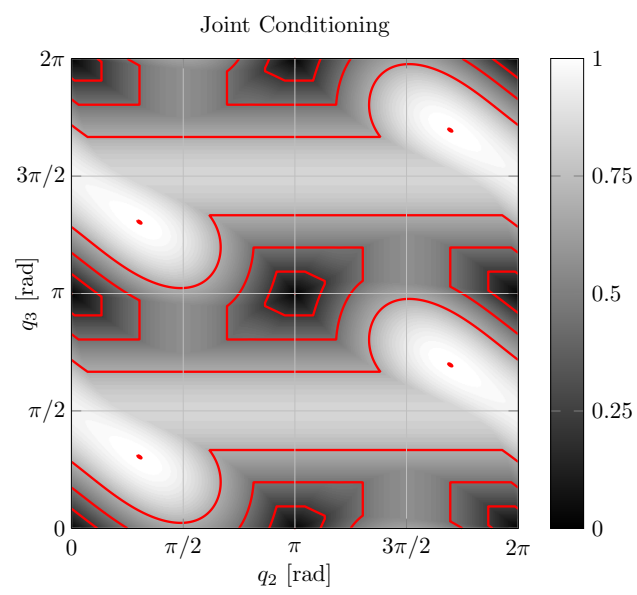

Figure 3.6: Contour plot of joint conditioning, the gray scale indicates the maximum value of the subdeterminants of $\mathbf{J}$. The red lines indicate level sets for $0.25,0.5$ and 0.75 . The dots indicate 1.0 conditioning. Zero (0) conditioning is found at $q_{2}=n \pi, q_{3}=m \pi, \forall n, m \in \mathbb{Z}$ at the dark areas. Note that the space repeats itself every $2 \pi \mathrm{rad}$.

A way to find a vector of nullspace motion is to find a projector matrix $\mathbf{P}(\mathbf{q})$ that can project any vector $\mathbf{w} \in \mathbb{R}^{4}$ onto the nullspace:

$$
\begin{aligned}
\mathbf{0} & =\mathbf{J}(\mathbf{q}) \dot{\mathbf{q}}_{n s}=\mathbf{J}(\mathbf{q}) \mathbf{w}-\overbrace{\mathbf{J}(\mathbf{q}) \mathbf{J}^{\dagger}(\mathbf{q})}^{\mathbf{I}_{3 \times 3}} \mathbf{J}(\mathbf{q}) \mathbf{w} \\
& =\mathbf{J}(\mathbf{q})\left(\mathbf{I}_{4 \times 4}-\mathbf{J}^{\dagger}(\mathbf{q}) \mathbf{J}(\mathbf{q})\right) \mathbf{w}=\mathbf{J}(\mathbf{q}) \mathbf{P}(\mathbf{q}) \mathbf{w} \\
\mathbf{P}(\mathbf{q}) & =\mathbf{I}_{4 \times 4}-\mathbf{J}^{\dagger}(\mathbf{q}) \mathbf{J}(\mathbf{q}),
\end{aligned}
$$

with $\mathbf{I}_{4 \times 4}$ a 4-by-4 identity matrix.

\subsubsection{Building the Nullspace-Configuration-Set}

Because $\mathbf{P}(\mathbf{q})$ is a projection matrix, it must have one eigenvector $\mathbf{u}_{\mathcal{N}}(\mathbf{q})$ for which the following holds:

$$
\mathbf{P}(\mathbf{q}) \mathbf{u}_{\mathcal{N}}(\mathbf{q})=\lambda \mathbf{u}_{\mathcal{N}}(\mathbf{q})=\mathbf{u}_{\mathcal{N}}(\mathbf{q}),
$$




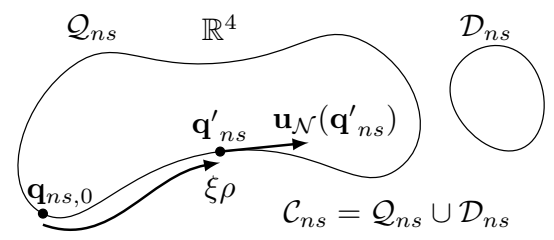

Figure 3.7: Integrating (3.15) from some starting point $\mathbf{q}$ will give the connected nullspace-configuration curve. The integration distance $\xi \rho$ leads to a new configuration $\mathbf{q}^{\prime}$ in $\mathcal{Q}_{n s}$ which has a direction vector $\mathbf{u}_{\mathcal{N}}\left(\mathbf{q}^{\prime}\right)$. There might be a disconnected nullspace-configuration-set $\mathcal{D}_{n s}$ which cannot be reached by traversing through $\mathcal{Q}_{n s}$.

with $\lambda=1$. All other eigenvectors are orthogonal to $\mathbf{u}_{\mathcal{N}}(\mathbf{q})$ and have eigenvalues of 0 . Geometrically this means that $\mathbf{u}_{\mathcal{N}}(\mathbf{q})$ points in the direction of the set of 'nullspace configurations' $\mathcal{C}_{n s}(\boldsymbol{\Gamma})$, i.e. it is a basis of $\mathcal{N}(\mathbf{J}(\mathbf{q}))$. This shows that the nullspace-configuration-set $\mathcal{C}_{n s}(\boldsymbol{\Gamma})$ is a curve in $\mathbb{R}^{4}$ jointspace. Hence, we will sometimes refer to $\mathcal{C}_{n s}(\boldsymbol{\Gamma})$ as the $\mathcal{C}_{n s}$-curve.

The total nullspace-configuration-set (also called the self-motion-manifold) $\mathcal{C}_{n s}(\Gamma)$, is given by:

$$
\mathcal{C}_{n s}(\boldsymbol{\Gamma})=\left\{\mathbf{q}_{n s} \in \mathbb{R}^{4}: \mathcal{F}\left(\mathbf{q}_{n s}\right)={ }^{g} \mathbf{R}_{u a}(\boldsymbol{\Gamma})\right\},
$$

with $\mathcal{F}(\cdot)$ the direct forward kinematic function from (3.3). For readability we will mostly omit the dependency on $\Gamma$ in further text.

The $\mathcal{C}_{n s}$ can be composed of two non-connected subsets, between which there would be no smooth transition. For the exoskeleton this means that the human arm would have to move 'out of the way' to be able to traverse between both sets of nullspace configurations.

For any $\mathbf{q}_{n s, 0} \in \mathcal{C}_{n s}(\boldsymbol{\Gamma})$, we can find local elements in the subset

$$
\mathcal{Q}_{n s}\left(\boldsymbol{\Gamma}, \mathbf{q}_{n s, 0}\right)=\left\{\mathcal{C}_{n s}(\boldsymbol{\Gamma}): \text { connected to } \mathbf{q}_{n s, 0}\right\} \subseteq \mathcal{C}_{n s}(\boldsymbol{\Gamma})
$$

These configurations are calculated by solving the differential equation (starting at some $\mathbf{q}_{n s, 0}$, as shown in Fig. 3.7)

$$
\frac{d \mathbf{q}_{n s}}{d \xi}=\rho \mathbf{u}_{\mathcal{N}}\left(\mathbf{q}_{n s}\right)
$$

This is trivially proved by remembering that $\mathbf{u}_{\mathcal{N}}\left(\mathbf{q}_{n s}\right)$ is a basis of $\mathcal{N}(\mathbf{J}(\mathbf{q}))$. 
The other 'half' of the $\mathcal{C}_{n s}$ that is not reachable we call the disjointed region $\mathcal{D}_{n s}$, defined as

$$
\mathcal{D}_{n s}\left(\boldsymbol{\Gamma}, \mathbf{q}_{n s, 0}\right)=\mathcal{C}_{n s}(\boldsymbol{\Gamma}) \backslash \mathcal{Q}_{n s}\left(\boldsymbol{\Gamma}, \mathbf{q}_{n s, 0}\right) \subset \mathcal{C}_{n s}(\boldsymbol{\Gamma})
$$

For some $\Gamma$ we have that $\mathcal{D}_{n s}=\emptyset$.

\subsubsection{Properties of $\mathcal{C}_{n s}$}

When plotting the $\mathcal{C}_{n s}$-curves through (3.15) we notice 'regions' in jointspace with different 'behavior'. The bounds of these regions (separatrices) appear to be the $\mathcal{Q}_{n s}\left(\mathbf{q}_{n s, 0}\right)$-curves of $\mathbf{q}_{n s, 0}$ that contain $q_{2}=n \pi, q_{3}=m \pi, \forall n, m \in \mathbb{Z}\left(q_{1}\right.$ and $q_{4}$ values do not matter). The separatrices pass through rank-2 (fully singular) configurations. Therefore we will call these bounds singularity-separatrices.

The singularity-separatrices can be projected on the $q_{2}-q_{3}$-plane, as shown in Fig. 3.8a. Investigation shows six regions of nullspace-configuration curves that show three different kinds of behavior, dependent on values of $\boldsymbol{\Phi}$.

The regions and behaviors for our specific design are as follows (neglecting $q_{4}$, because it is only linked to axial rotation of the arm):

- Type Ia and Ib solutions: two disjointed closed loops $\mathcal{Q}_{n s}$ and $\mathcal{D}_{n s}$ in $q_{2}-q_{3}$ but continuously connected in $q_{1}$. The total shape is helical.

- Type IIa and IIb solution: a single (i.e. $\mathcal{D}_{n s}=\emptyset$ ) complete closed loop $\mathcal{C}_{n s}$ in $q_{1}-q_{2}-q_{3}$.

- Type III solution: two disjointed curves $\mathcal{Q}_{n s}$ and $\mathcal{D}_{n s}$, continuously connected in $q_{1}-q_{2}$, but limited in domain in $q_{3}$.

Typical projected-solutions for all three types of $\mathcal{C}_{n s}$-curves are shown in Fig. 3.9 The singularity-separatrices have a clear meaning when considering the human arm-movement. They are elevation circles on the elbow-sphere, but rotated over two angles $\boldsymbol{\Theta}$. The circles are at relative elevation angles (relative to the rotated north- and south-'poles' on the sphere) $15^{\circ}, 75^{\circ}, 105^{\circ}$ and $165^{\circ}$ (dependent on $\Phi)$. This gives 5 regions for Ia, IIa, III, IIb and Ib solution types. An illustration of these circles is given in Fig. 3.8b.

We can draw several conclusions from the separatrix-planes and the nullspaceconfiguration-curves:

1. It shows that the gimbal system can only become singular when the arm is on such a curve and almost singular when close to such a curve. Hence, 


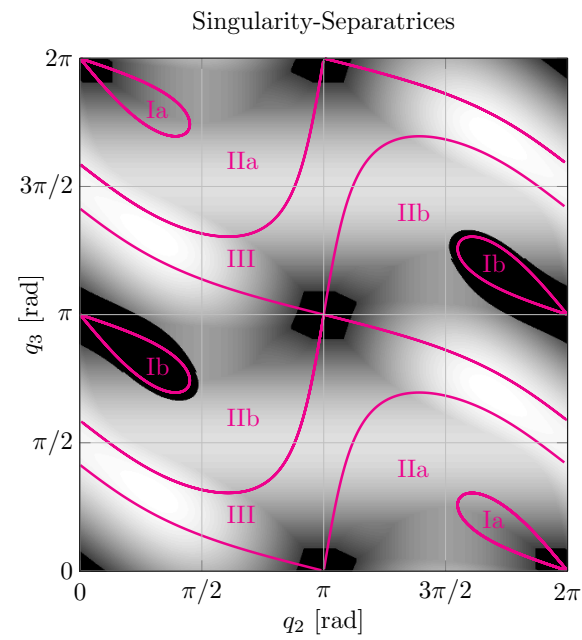

(a)

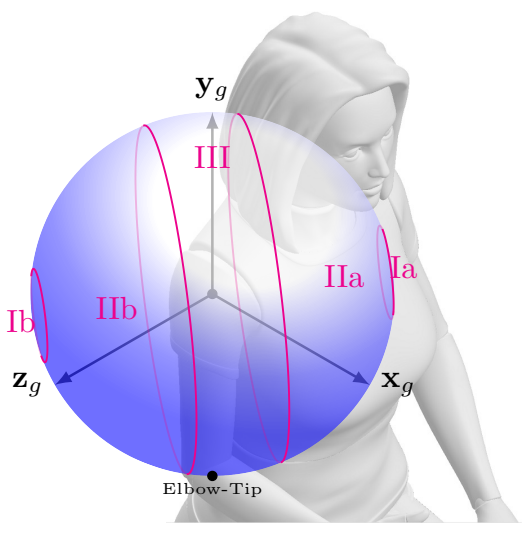

(b)

Figure 3.8: a) Singularity-separatrices: The $q_{2}-q_{3}$ plane showing the conditioning and moving arm collision constraints. Superimposed are the singularityseparatrices in which nullspace-configurations curves will always stay. Each region is numbered dependent on the type of behavior of the curve. It can be seen that the Ib region always overlaps with the collision of the upper arm with the first motor. It should be noted that the space repeats itself every $2 \pi$ rad. b) The human elbow-tip can reach points on a sphere. On this sphere, the nullspace-separatrices are circles (magenta). Only if the elbow is on such a circle, the exoskeleton can possibly become fully singular. The elbow-tip point is illustrated at the bottom of the sphere.

we are only in danger of poor conditioning close to these curves, and we can focus more on collision avoidance outside of these neighborhoods.

2. The IIa and IIb solutions are the only ones that have $\mathcal{D}_{n s}=\emptyset$. Hence, if we want to traverse between disjointed regions in Ia, Ib or III, we could do so through IIa or IIb.

3. On purpose we placed the Ib solution region outside of the human-workspace, since that region will always overlap with the collision of the human arm with the first motor. 


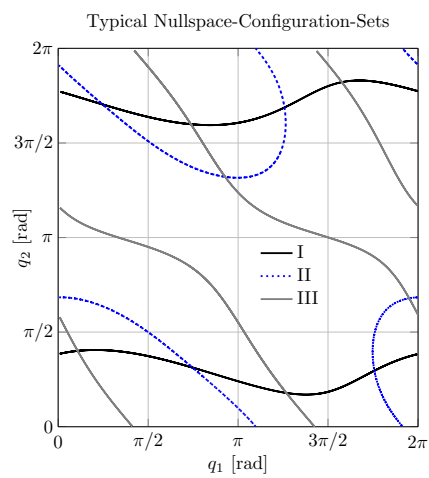

(a)

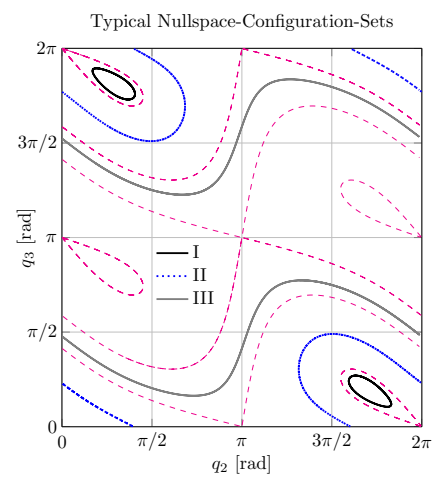

(b)

Figure 3.9: Typical shapes of null-space-configuration sets, a) $q_{1}-q_{2}$-plane b) $q_{2}-q_{3}$-plane. The shapes are invariant to $q_{1}$ and $q_{4}$, but will translate along the $q_{2}-q_{3}$ view if $q_{1}$ changes. The separatrices are shown as magenta colored dashed lines.

\subsection{PROPOSED INVERSE KINEMATICS METHOD}

We require a method that uses (3.8), the minimum norm velocities in combination with nullspace velocities, to move to a configuration that still satisfies (3.4), but should not violate any collision or conditioning constraint.

A candidate solution is gradient projection of the form

$$
\dot{\mathbf{q}}_{n s}=\mathbf{P}(\mathbf{q}) \nabla f(\mathbf{q}),
$$

with $\mathbf{P}(\mathbf{q})$ the projector described in (3.11), $f(\mathbf{q})$ a scalar potential function with a global minimum (global in the sense of a $2 \pi$ repeating space would imply it is local in a hypercube, but there is only a single potential minimum in such a hypercube) in $\mathbb{R}^{4}$ dependent on exoskeleton conditioning and collision avoidance.

The gradient projection method, in general, finds only locally optimal configurations in $\mathcal{Q}_{n s}$ since convergence ends when $\mathbf{u}_{\mathcal{N}}(\mathbf{q})$ and $\nabla f(\mathbf{q})$ are orthogonal. The $\mathcal{Q}_{n s}$ is a highly complex function of $\mathbf{q}$. To have more control over what configuration the exoskeleton converges to, a single global minimum on $\mathcal{Q}_{n s}$ would have to be constructed with the function $f(\mathbf{q})$ to apply (3.17).

Generating the potential $f(\mathbf{q})$ to accomplish this task is very difficult, in general, because it requires full knowledge and parameterization of $\mathcal{Q}_{n s}$. Therefore, we relieve this difficulty by designing a potential on the $1 \mathrm{D} \mathcal{Q}_{n s}$. We can then 


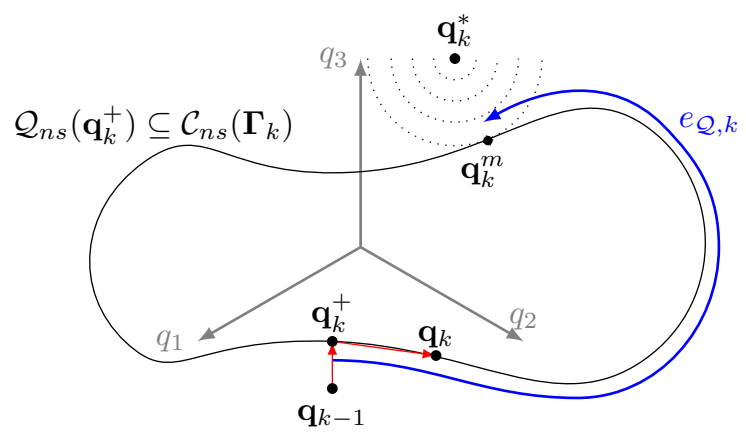

Figure 3.10: We start in the calculated configuration from the previous time-step $\mathbf{q}_{k-1}$. The human imposes a shoulder velocity $g_{\Omega_{k}}$. A minimum-norm solution gives a new configuration $\mathbf{q}_{k}^{+}$. This configuration has a connected nullspaceconfiguration-set $\mathcal{Q}_{n s}\left(\mathbf{q}_{k}^{+}\right)$, which gives exoskeleton configurations equally valid to hold the shoulder orientation $\boldsymbol{\Gamma}_{k}$. The point $\mathbf{q}_{k}^{m}$ on $\mathcal{Q}_{n s}\left(\mathbf{q}_{k}^{+}\right)$is closest to attractor configuration $\mathbf{q}_{k}^{*}$ in an Euclidean sense. The scalar error $e_{\mathcal{Q}, k}$ is determined between $\mathbf{q}_{k}^{m}$ and $\mathbf{q}_{k}^{+}$along $\mathcal{Q}_{n s}\left(\mathbf{q}_{k}^{+}\right)$. Negative feedback along $\mathcal{Q}_{n s}\left(\mathbf{q}_{k}^{+}\right)$will make the configuration always converge to $\mathbf{q}_{k}^{m}$.

converge to some minimum on $\mathcal{Q}_{n s}$, which is globally minimal. Instead of designing the potential, we directly apply the gradient as a negative feedback on the $1 \mathrm{D} \mathcal{Q}_{n s}$, ensuring linear (bang-bang control) or exponential (linear control) convergence in $\mathcal{Q}_{n s}$ to some preferred configuration. Feedback will reduce the scalar error along $\mathcal{Q}_{n s}$. The length of $\mathcal{Q}_{n s}$ changes as a function of $\Gamma$, but this cannot counteract convergence. Since the length of $\mathcal{Q}_{n s}$ has an upper bound, either because it is shaped as a closed loop, or because the jointspace repeats every $2 \pi$ $\mathrm{rad}$, the nullspace configurations will converge to the minimal configuration as time $t \rightarrow \infty$. The changing length of $\mathcal{Q}_{n s}$, due to the change in spatial metric, acts merely as a disturbance that can be completely rejected. Because the algorithm will be implemented in software, we will use a discrete time version of (3.7) and (3.8) for the construction of the algorithm.

\subsubsection{Explanation of the Algorithm}

The algorithm will be described step by step. An illustration of these steps is given in Fig. 3.10. Every discrete time-step, all steps of the algorithm have to be executed. 
1. From an admittance control model we get a bounded $g \boldsymbol{\Omega}_{k}$, which would move us to the current arm configuration $\Gamma_{k}$.

2. We perform a minimum-norm 'jump' to a valid configuration solution $\mathbf{q}_{k}^{+}$ for the next time step (Eq. (3.7) and (3.19)). This solution is a valid element of the next nullspace-configuration-set $\mathcal{Q}_{n s}\left(\mathbf{q}_{k}^{+}\right) \subseteq \mathcal{C}\left(\boldsymbol{\Gamma}_{k}\right)$.

3. We determine an 'attractor' $\mathbf{q}_{k}^{*}$ (this can be a point, line/curve or (hyper)plane). This attractor could be dependent on several things: the current arm-region of $\boldsymbol{\Gamma}_{k}$ (region I,II or III) and previous configuration $\mathbf{q}_{k-1}$ and current candidate $\mathbf{q}_{k}^{+}$. (Eq. (3.18))

4. We calculate the complete connected $\mathcal{Q}_{n s}\left(\mathbf{q}_{k}^{+}\right)$belonging to $\mathbf{q}_{k}^{+}$(Eq. (3.15) and Eq. (3.20)).

5. We find the point $\mathbf{q}_{k}^{m}$ (called the minimal-point) on the nullspace-configurationset that has the closest Euclidean distance $\delta(\xi)$ to the attractor. (Eq. (3.21) and (3.22))

6. The scalar feedback-error $e_{\mathcal{Q}, k}$ determined along the connected nullspaceconfiguration-set $\mathcal{Q}_{n s}\left(\mathbf{q}_{k}^{+}\right)$by determining the distance along the curve from integrating from $\mathbf{q}_{k}^{+}$to $\mathbf{q}_{k}^{m}$. This is done by using (Eq. 3.15) and finding the integration limit $\xi^{m}$ that minimizes $\delta(\xi)$. (Eq. (3.22) and (3.23))

7. From the error $e_{\mathcal{Q}, k}=\xi^{m} \rho$ a nullspace velocity (or jump) is determined along the nullspace-configuration-set, using $\mathbf{u}_{\mathcal{N}}\left(\mathbf{q}_{k}^{+}\right)$and feedback gain $K$. If the used time step $T$ is small enough, we can assume we end on a valid element in $\mathcal{Q}_{n s}\left(\mathbf{q}_{k}^{+}\right)$. (Eq. (3.24))

8. The total combined minimum-norm and nullspace jump is limited properly to make sure no single element exceeds maximum joint velocity. (Eq. (3.24))

9. By numerical integration (Euler integration) the current configuration is updated to the new configuration. (Eq. (3.25)) 
The algorithm is summarized as follows:

$$
\begin{aligned}
\mathbf{q}_{k}^{*} & =f\left(\mathbf{q}_{k-1}, \mathbf{\Gamma}_{k}\right) \\
\mathbf{q}_{k}^{+} & =\mathbf{q}_{k-1}+T \mathbf{J}^{\dagger}\left(\mathbf{q}_{k-1}\right)^{g} \mathbf{\Omega}_{k} \\
\mathcal{Q}_{n s}\left(\mathbf{q}_{k}^{+}\right) & =\text {use Eq. }(3.14) \text { and Eq. }(3.15) \\
\delta(\xi) & =\text { Eucl. dist. betw. } \mathbf{q}_{k}^{*} \text { and } \mathcal{Q}_{n s}\left(\mathbf{q}_{k}^{+}\right) \\
\xi^{m} & =\underset{\xi \in \mathbb{R}}{\operatorname{argmin} \delta} \delta) \\
e_{\mathcal{Q}, k} & =\xi^{m} \rho \\
\dot{\mathbf{q}}_{k} & =\operatorname{sat}\left[\mathbf{J}^{\dagger}\left(\mathbf{q}_{k-1}\right)^{g} \mathbf{\Omega}_{k}-K e_{\mathcal{Q}, k} \mathbf{u}_{\sigma}\left(\mathbf{q}^{+}\right)\right] \\
\mathbf{q}_{k} & =\mathbf{q}_{k-1}+T \dot{\mathbf{q}}_{k},
\end{aligned}
$$

with $T$ the sample time of the algorithm and sat $[\cdot]$ a proper saturation function based on the maximum element. Some practical remarks on this algorithm can be found in the supplementary material.

Instead of solving the difficult problem of designing a potential function $f(\mathbf{q})$ for gradient projection, we have changed the problem to 1) choosing a simple $\mathbf{q}_{k}^{*}$, and 2) executing a computationally more intensive task of calculating parts of $\mathcal{Q}_{n s}\left(\mathbf{q}_{k}^{+}\right)$using Eq. (3.14) and Eq. (3.15).

\subsubsection{Choosing Attractor $\mathbf{q}_{k}^{*}$}

The challenge in the current method lies in generating an attractor $\mathbf{q}_{k}^{*}$. We have to generate an attractor that ensures (in combination with feedback) the solutions to stay away from collision and poor conditioning. We pick a possible simple choice, valid for our design choices $\boldsymbol{\Phi}, \boldsymbol{\Theta}$ and geometry. In our case, the attractor is a hyper-plane described by the implicit plane equation $\left(q_{2}-0.6 \pi\right)=0$, meaning $q_{1}, q_{3}, q_{4}$ can take any value. Choosing this hyper-plane ensures attraction of $q_{2}$ to $0.6 \pi \mathrm{rad}$, staying away from body collisions and poor conditioning. If the value of $0.6 \pi$ cannot be achieved for $q_{2}$ (either it is not a valid element of the connected $\mathcal{Q}_{n s}$, or it would violate the velocity constraints), it will try to be 'close'. Our attractor is invariant to the solution-region and arm/joint velocities, although it need not be.

We want to emphasize that this choice for $\mathbf{q}_{k}^{*}$ may not be the best, but is used to show that the problem can now be solved, even with a simple choice for the 
attractor. A better, more dynamic, attractor could try to improve exoskeleton conditioning to achieve lower joint velocities.

\subsubsection{Claims of Quasi-Static Convergence}

An informal proof of convergence of our method is given in a quasi-static sense, i.e. when the exoskeleton starts in a configuration conforming to constraints, not obstructed by constraints, and the human moves infinitely slowly (or: the feedback gain is higher than the human movement speed). In this way, the IK method converges to the minimal point $\mathbf{q}_{k}^{m}$. This 'theorem' can be used afterwards to find conditions for dynamic convergence in Sec. 3.4.4.

First, we denote all possible null-space-configurations in $\mathcal{Q}_{n s}$ that are accessible or allowed (because they do not violate a conditioning or collision constraint), and are directly connected to our current configuration $\mathbf{q}_{k}$ as the allowed compact subset:

$$
\mathcal{A}_{n s}\left(\boldsymbol{\Gamma}, \mathbf{q}_{k}\right)=\left\{\mathbf{q}_{n s} \in \mathcal{Q}_{n s}\left(\boldsymbol{\Gamma}, \mathbf{q}_{k}\right): \text { conforming to constraints }\right\}
$$

Such subsets we call ' $\mathcal{A}_{n s}$-loops'.

Secondly, we need two prerequisites to make any convergence claims:

Prerequisite 1: The rotational velocity $g \Omega$ of the shoulder, imposed by the human, is bounded. We assume a finite $L_{2}$ norm of $\left\|\Omega_{\text {max }}\right\|_{2}$.

Prerequisite 2: The rotations of the $\operatorname{arm} \Gamma$ are always element of a smaller compact set, the human workspace $\mathcal{W}_{h}$, than the complete set of all rotations, i.e. $\Gamma \in \mathcal{W}_{h} \subset S O(3)$.

Prerequisite 1 is required, otherwise a quasi-static proof of convergence has no value. Prerequisite 2 is required, because vanishing $\mathcal{A}_{n s}$-loops do occur outside of the human workspace (see more explanation about vanishing $\mathcal{A}_{n s}$-loops in Claim 2 below), which would be problematic.

Consequently, we make the following claims which, if they all hold, ensure the system never violates conditioning and collision constraints in a quasi-static sense.

Claim 1: For any arm configuration there is always a minimal point $\mathbf{q}_{k}^{m}$ that does not violate any constraint.

Claim 2: The minimal point $\mathbf{q}_{k}^{m}$ is never inside a $\mathcal{A}_{n s}$-loop that vanishes completely into a constraint (i.e. $\mathcal{A}_{n s}\left(\boldsymbol{\Gamma}, \mathbf{q}_{n s}\right)=\emptyset$ ) for 'some (unknown) worst arm motion' $g \Omega$ that would make this $\mathcal{A}_{n s}$-loop vanish. 
The proof is informal since both claims can only be shown to be true by visual inspection. Claim 1 is achieved by proper choice of $\mathbf{q}_{k}^{*}$. It holds, since the hyperplane $\mathbf{q}_{k}^{*}=0.6 \pi$ intersects with $\mathcal{Q}_{n s}$ always in region III, and for part of the arm configurations in regions IIa and IIb. If the plane does not intersect, the projected shape of $\mathcal{Q}_{n s}$ onto $q_{1}-q_{2}$ in the region $q_{2} \in[0, \pi]$ is fully convex outside the constraints in regions IIa, IIb and Ia. Therefore, there is a single minimal point conforming to all constraints. The region Ib has no feasible solutions by design, due to motor 1 collision, and is placed outside of $\mathcal{W}_{h}$, and is not a problem. Claim 2 is achieved by by proper design choices of $\boldsymbol{\Phi}, \boldsymbol{\Theta}$. It holds because: a) In region Ia, no arm movement $g \Omega$ can make the $\mathcal{A}_{n s}$-loop vanish. b) Similarly, for the part of the IIa region, there is no possibility for an $\mathcal{A}_{n s}$-loop to vanish into a constraint. c) In region III and parts of IIa and IIb, the projected shape of $\mathcal{Q}_{n s}$ is almost diagonal across the $q_{1}-q_{2}$ plane. Therefore, there is always an intersecting solution $\mathbf{q}_{k}^{m}=\mathbf{q}_{k}^{*}$. d) In region IIb, the only remaining $\mathcal{A}_{n s}$-loop almost vanishes when approaching region $\mathrm{Ib}$, but it never does. Although solution space $\mathcal{A}_{n s}$ becomes small, it remains convex in projection on $q_{1}-q_{2}$, with a single $\mathbf{q}_{k}^{m}$.

\subsubsection{Condition for Dynamic Convergence}

Given the claims that the method will quasi-statically converge, it is possible to derive sufficient conditions for convergence in a dynamic sense.

The differential kinematics from (3.5), can be rewritten by performing an a singular value decomposition as follows:

$$
{ }^{g} \boldsymbol{\Omega}=\mathbf{J}(\mathbf{q}) \dot{\mathbf{q}}=\mathbf{U} \Sigma \mathbf{V}^{T} \dot{\mathbf{q}},
$$

with

$$
\Sigma=\left[\operatorname{diag}\left(\left[\sigma_{1}, \sigma_{2}, \sigma_{3}\right]\right) \mathbf{0}_{3}\right] .
$$

Then the minimum norm solution to (3.7) is described by

$$
\dot{\mathbf{q}}_{m n}=\mathbf{J}^{\dagger}(\mathbf{q})^{g} \boldsymbol{\Omega}=\mathbf{V} \Sigma^{+} \mathbf{U}^{T g} \boldsymbol{\Omega},
$$


with

$$
\Sigma^{+}=\left[\begin{array}{c}
\operatorname{diag}\left(\left[\sigma_{1}^{-1}, \sigma_{2}^{-1}, \sigma_{3}^{-1}\right]\right) \\
\mathbf{0}_{3}^{T}
\end{array}\right] .
$$

In (3.27), $\sigma_{j}^{-1}(j=1,2,3)$ are the principal axes of the ellipsoid formed by the singular values of $\mathbf{J}^{\dagger}(\mathbf{q})$. This ellipsoid governs the scaling of the input vector $8 \boldsymbol{\Omega}$ to the jointspace. If we set $\sigma_{\min }=\min \left(\left[\sigma_{1}, \sigma_{2}, \sigma_{3}\right]\right)$, the minimum norm solution is always within a sphere with the largest ellipsoid radius $\sigma_{\text {min }}^{-1}$ :

$$
\left\|g \mathbf{\Omega}_{\text {max }} \sigma_{\text {min }}^{-1}\right\|_{2} \geq\left\|\dot{\mathbf{q}}_{\text {mn,max }}\right\|_{2} .
$$

This holds because $\mathbf{V}, \mathbf{U}$ and its transposes are unitary and do not scale vectors.

The minimum-norm solution can drop the new configuration anywhere inside, or onto, the sphere. In a worst case, this is exactly $\left\|g \Omega_{\text {max }} \sigma_{\text {min }}^{-1}\right\|_{2} T$ away from the minimal point $\mathbf{q}_{k}^{m}$. Therefore, we need the nullspace-velocity-norm to be greater than the minimum-norm to bring us always closer towards $\mathbf{q}_{k}^{m}$ along $\mathcal{Q}_{n s}$.

To achieve this, we need that

$$
\left\|\dot{\mathbf{q}}_{n s}\right\|_{2} \geq\left\|g \boldsymbol{\Omega}_{\max } \sigma_{\min }^{-1}\right\|_{2} .
$$

Due to our design choices, this is equivalent to

$$
\left\|\mathbf{u}_{\mathcal{N}} K e_{\mathcal{Q}}\right\|_{2} \geq\left\|g \Omega_{\text {max }} \sigma_{\text {min }}^{-1}\right\|_{2} \text {. }
$$

Since $\left\|\mathbf{u}_{\mathcal{N}}\right\|_{2}=1$ and by default $K \geq 0$ and $\sigma_{\text {min }} \geq 0$, we can rewrite this condition as

$$
K\left|e_{\mathcal{Q}}\right| \geq\left\|^{g} \boldsymbol{\Omega}_{\text {max }}\right\|_{2} \sigma_{\text {min }}^{-1} .
$$

Which gives as condition for our feedback gain:

$$
K \geq \frac{\left\|^{g} \boldsymbol{\Omega}_{\max }\right\|_{2}}{\left|e_{\mathcal{Q}}\right|} \sigma_{\text {min }}^{-1},
$$




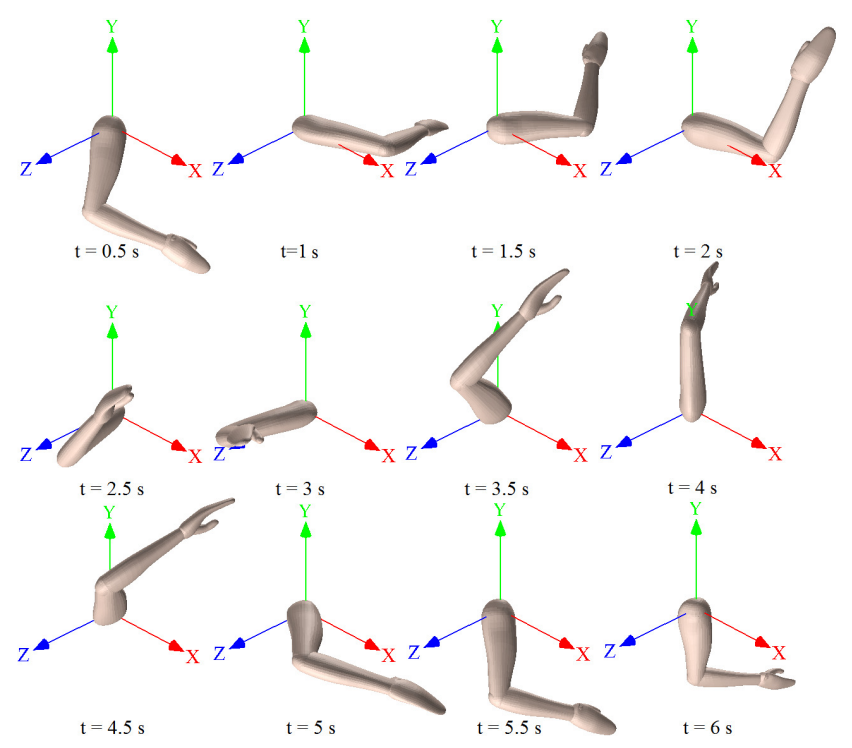

Figure 3.11: Benchmark arm movement. The arm starts in the zero configuration at $t=0$ s. All sequential poses are shown at time intervals of $0.5 \mathrm{~s}$.

such that a valid nullspace velocity solution condition for dynamic convergence is

$$
\dot{\mathbf{q}}_{n s} \geq\left\|^{g} \boldsymbol{\Omega}_{\max }\right\|_{2} \sigma_{\min }^{-1} \operatorname{sgn}\left(e_{\mathcal{Q}}\right) \mathbf{u}_{\mathcal{N}} .
$$

This nullspace velocity ensures a net convergence as discrete time $k \rightarrow \infty$. It also gives a maximally allowed arm velocity, dependent on exoskeleton joint motor capabilities.

Instead of the $\operatorname{sgn}(x)$-function, an alternative is the use of the $\tanh (\beta x)$, since $\lim _{\beta \rightarrow \infty} \tanh (\beta x)=\operatorname{sgn}(x)$. This ensures smoother behavior for small $e_{\mathcal{Q}}$, as long as for errors greater than approximately $0.1 \operatorname{rad} \tanh \left(\beta\left|e_{\mathcal{Q}}\right|\right) \approx 1$, we still have guaranteed convergence before hitting any constraint surface.

Also, to be less conservative, instead of $\left\|^{g} \Omega_{\text {max }}\right\|_{2}$ in (3.29), it is possible to use $\left\|^{g} \boldsymbol{\Omega}_{k}\right\|_{2}$ of the current time step. 


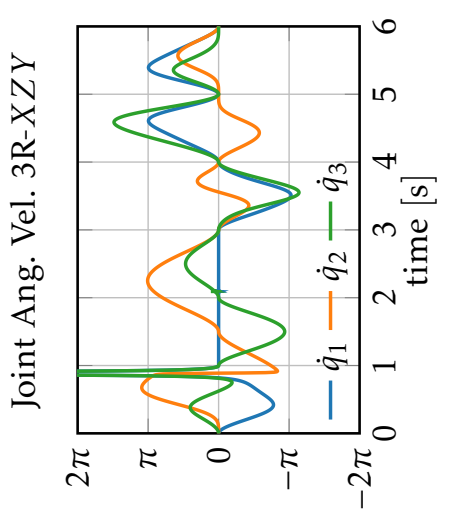

$[\mathrm{s} / \mathrm{pex}] \cdot[\partial \Lambda$ ле[noue

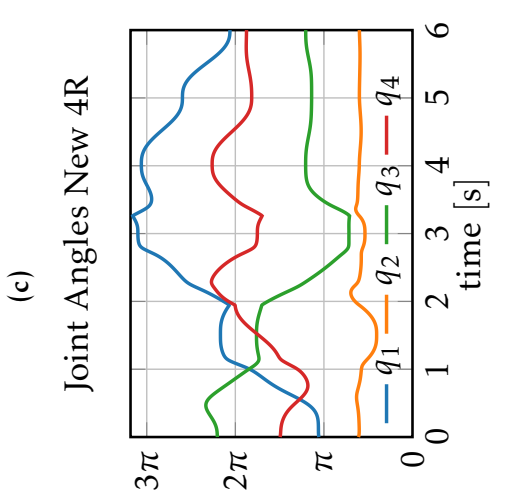

苛芯 đ: 竝 य गे क्षे के

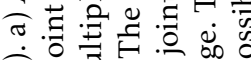
$\Phi$ 交.

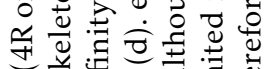
\&

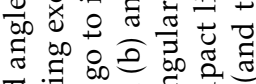
[pex] ว[8ие

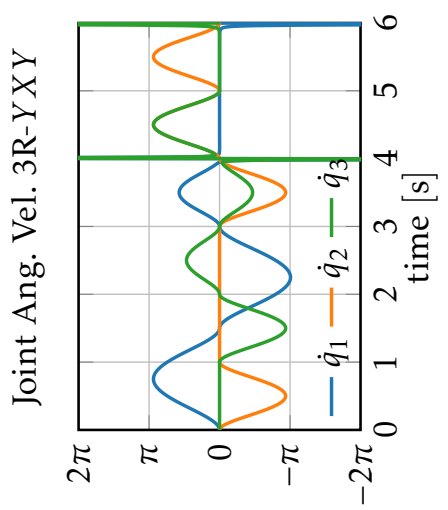

[s/pex] ' [әм ле[noue

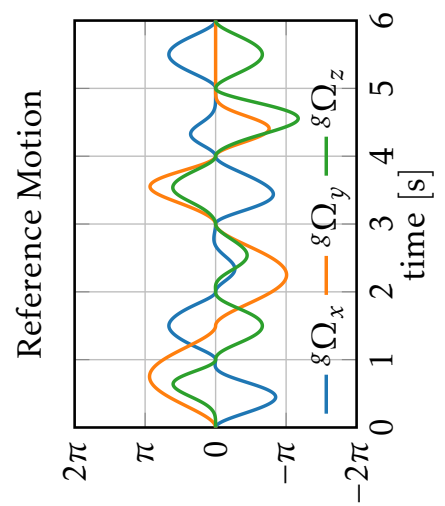

$[\mathrm{s} / \mathrm{pex}] \cdot$ тал ле[nosue

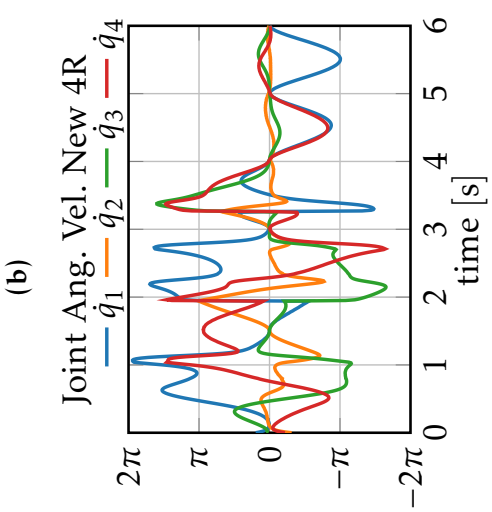

[s / pes] ' [әм ле[noue

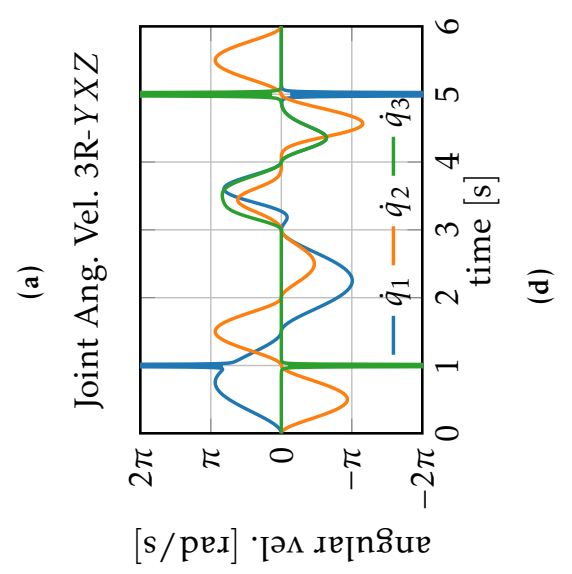
๙ิ + 先

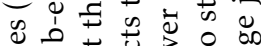
: Ð D东过

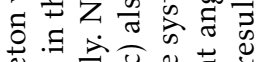
(2)

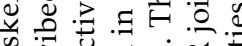

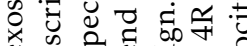

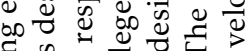
$\Xi$ क क थ 0

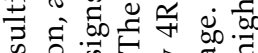
क्ष

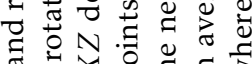
๙

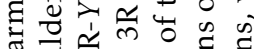

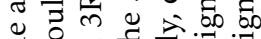
ङ प्ञ 0 व

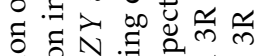

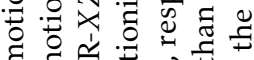

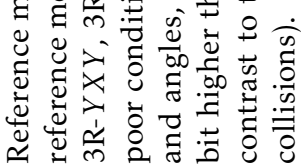
ते 


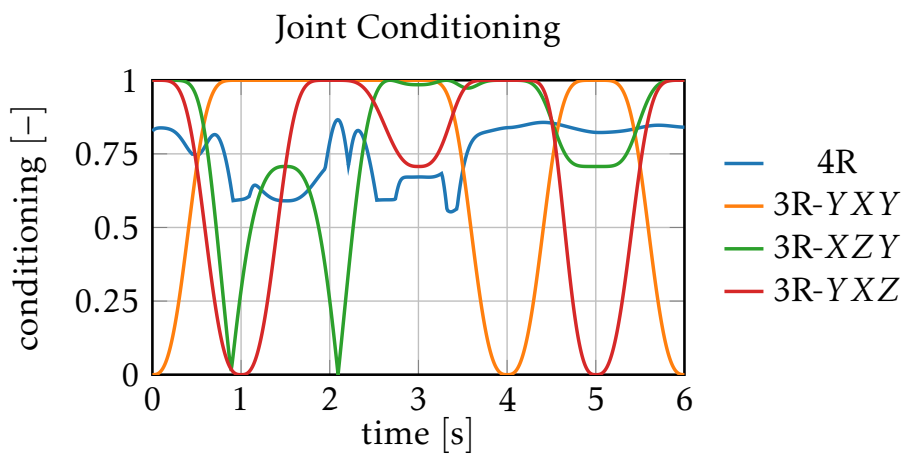

Figure 3.13: Conditioning of the $4 \mathrm{R}$ and $3 \mathrm{R}$ exoskeletons. The $3 \mathrm{R}$ exoskeletons have poor conditioning at certain arm configurations. The $4 \mathrm{R}$ system manages to have better overall conditioning, which helps in avoiding excessive joint velocities. The fact that the maximal conditioning is a bit less than for all $3 \mathrm{R}$ exoskeletons, is because the $4 \mathrm{R}$ exoskeleton is non-orthogonal.

\subsection{RESULTS}

\subsubsection{Analytical Benchmark}

In this section we show a validation of the method by following an analytical benchmark movement with the new $4 \mathrm{R}$ design. The conditioning and joint velocities are compared to common orthogonal 3R exoskeleton architectures given by rotation orders $Y X Y, X Z Y, Y X Z$ (stating the exoskeleton axis direction in the human's zero-pose), as is shown in [44] (see Chapter 2, Sec. 2.2.1 and 2.2.2).

We did not generate geometry for the 3R designs and could therefore not check for head, trunk and arm collisions in their case. We did, however, check for head, trunk and arm collisions for the new 4R design, since it was a prerequisite for the method to succeed and to be useful.

The benchmark motion is shown in Fig. 3.11 and Fig. 3.12a. It is composed of local rotations, transformed to the global frame, to generate a global arm velocity $g \Omega$. The benchmark motion is such that the $3 \mathrm{R}$ shoulders will pass through their singular configurations, resulting in very large joint velocities, approaching infinity.

The simulation for $3 \mathrm{R}$ and $4 \mathrm{R}$ was performed with $T=1 \mathrm{~ms}$. The used $4 \mathrm{R}$ IK method was the one in (3.29), but with $\tanh \left(5 e_{\mathcal{Q}}\right)$ instead of $\operatorname{sgn}\left(e_{\mathcal{Q}}\right)$. In Fig. $3.12 \mathrm{~b}-\mathrm{d}$ it is clearly visible that $3 \mathrm{R}$ shoulders all have troubling configurations at which singularity occurs, while the movement is within the human workspace. At these configurations the joint conditioning of the $3 \mathrm{R}$ designs be- 


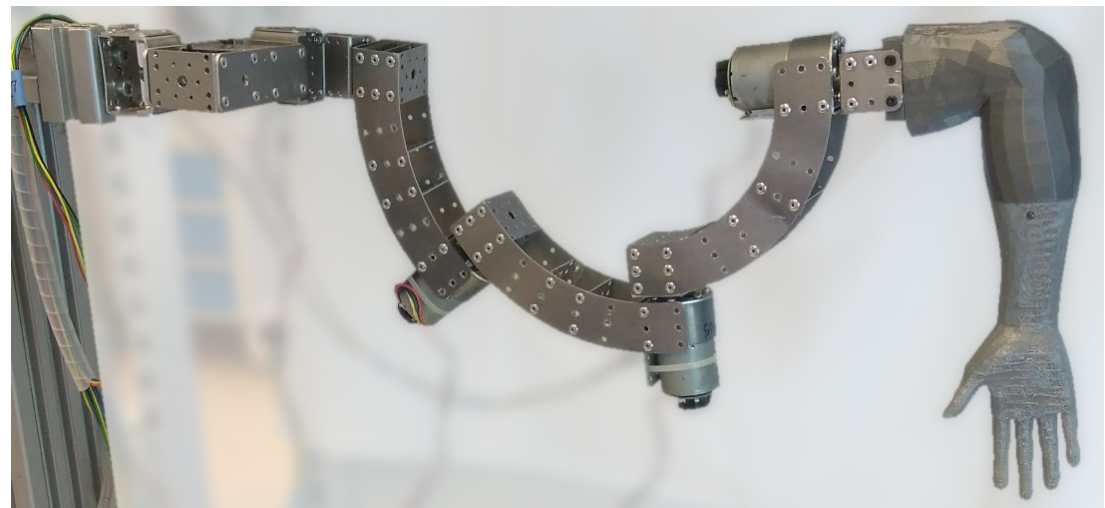

(a)

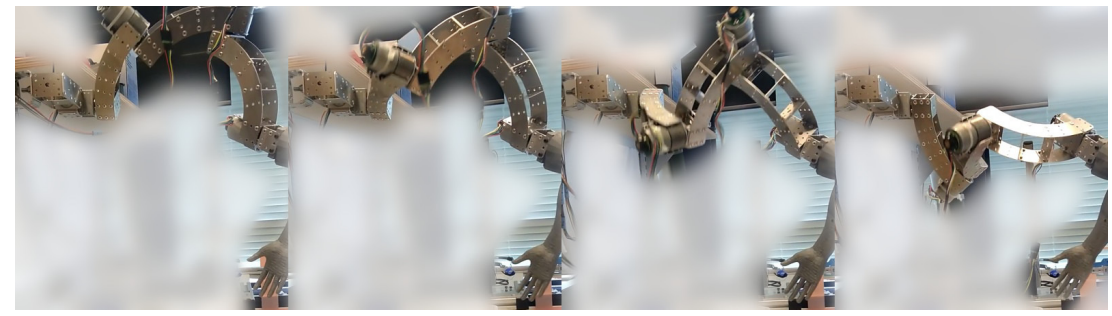

(b)

Figure 3.14: a) The used 1:2 scale setup shown conceptually in Fig. 3.1 and detailed in Table 3.1. Four brushed DC motors drive the four DOF. The setup has a single CoR where all joint axes intersect. b) From left to right: a self-motion of the joint, frames spaced approximately $0.3 \mathrm{~s}$ apart. Note that the arm hardly moves during self-motion of the joint. Any residual motion is due to incremental encoder angle calibration error and play in the motor transmission.

comes very poor, while the $4 \mathrm{R}$ design actively attempts to continually achieve proper conditioning (see Fig. 3.13).

\subsubsection{Test Setup}

A 1:2 scale test setup, shown conceptually in Fig. 3.1 and detailed in Table 3.1, is shown in Fig. 3.14. The four DOF are driven by Pololu D37 brushed DC motors with transmission ratios of 1:70, 1:50, 1:50, 1:19 for motors actuating axes $\omega_{1}$ to $\omega_{4}$ respectively. All four motors have 64 incremental encoder counts per 


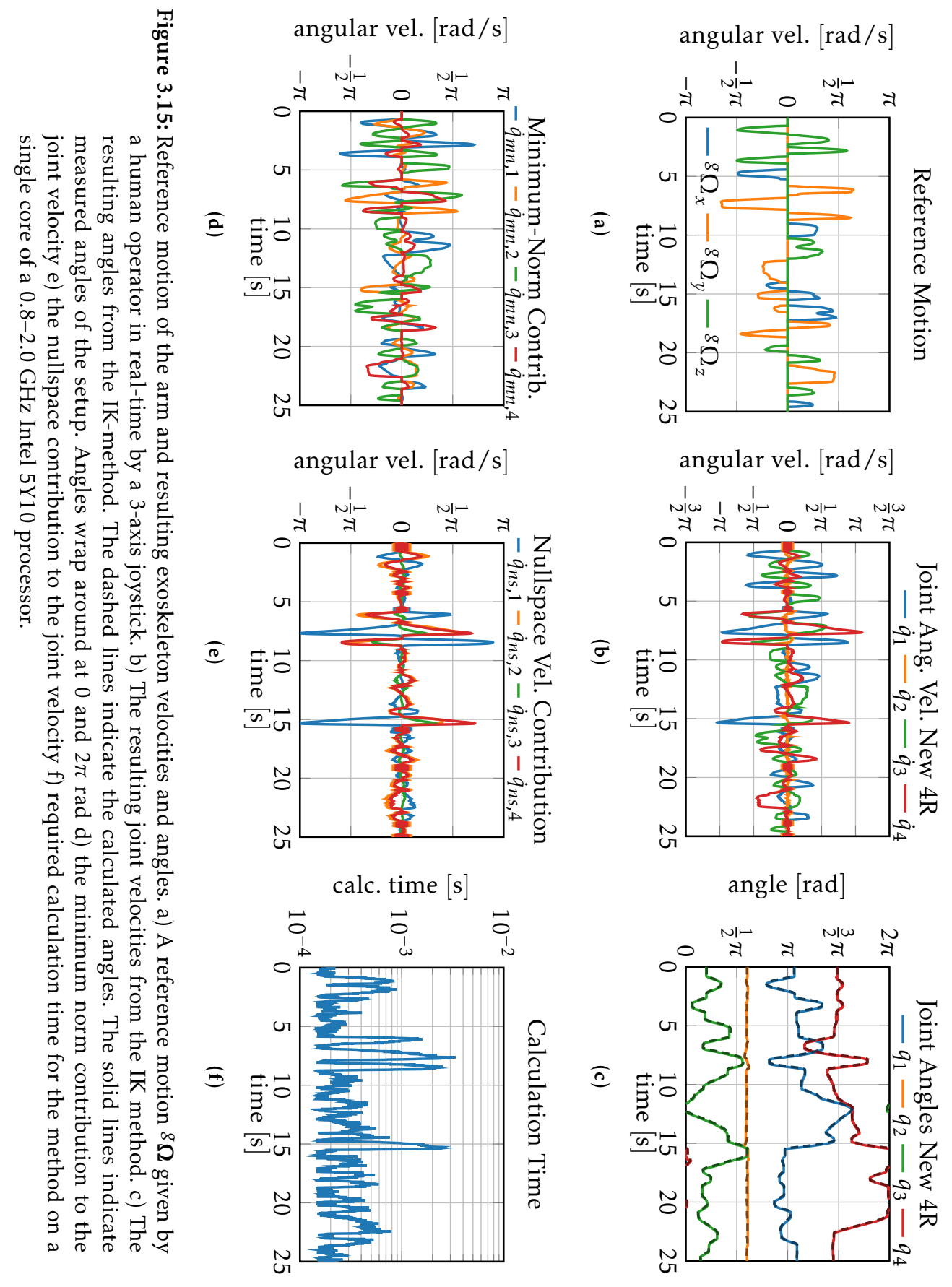




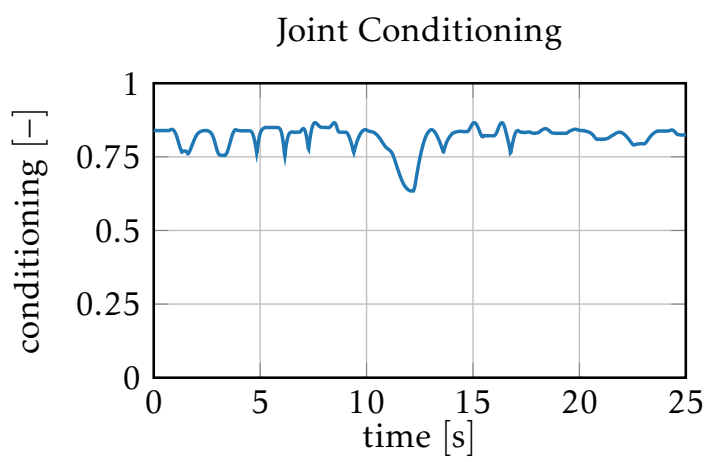

Figure 3.16: Conditioning of the $4 \mathrm{R}$ test setup during the motion.

motor revolution. The motors are driven with two Pololu VNH5019 Dual motor drivers with $9 \mathrm{~V}$ power supply. Low-level PID joint angle control runs at an update frequency of $1000 \mathrm{~Hz}$ and is implemented on an NXP FRDM K64F board (MK64FN1M0VLL12 microcontroller, running at $120 \mathrm{MHz}$ ) that sends PWM signals to the motor drivers. Instead of a force/torque sensor, the deflection of a 3-axis joystick (pitch, roll and yaw) is used to determine global arm velocity $g \Omega$. In a full size exoskeleton a force/torque sensor on the exoskeleton would measure human operator forces/torques. These torques would generate a local angular acceleration that would be integrated and transformed to a velocity vector expressed in the global frame. A 3D-printed 1:2 scale human arm is attached to be able to visualize axial arm configuration.

The IK algorithm used is the same as in Sec. 3.5.1. Code used to calculate results in Sec. 3.5.1 is compiled to a Matlab executable in Matlab 2015b on Microsoft Windows 10. This allows for single-threaded IK computations on a single core of a $0.8-2.0 \mathrm{GHz}$ Intel 5Y10 Dual-Core M processor. Calculated reference joint angles from the IK method are transmitted from Matlab over USB to the microcontroller at a rate of $50 \mathrm{~Hz}$.

The arm started in the human's zero-pose. A human operator gave a real-time desired arm velocity, in the global frame, to the system by moving the 3-axis joystick (see Fig. 3.15a). The resulting motion of the setup is shown in Fig. 3.15bc. The contribution of the minimum-norm solution and the added nullspace contribution is shown in Fig. 3.15d-e. It shows that the minimum-norm and nullspace solution have opposite signs when moving joint $q_{2}$. The software used in the setup checks whether it would violate any of the jointspace constraints outlined in Sec. 3.3.1 and would then stop the motion. This did, however, not 
happen during the testing motion. Conditioning of the joint was above 0.6 , as is shown in Fig. 3.16, similar to results in Fig. 3.13.

Computationally, the method was sufficient for real-time applications, as is shown in see Fig. 3.15f. Calculation times were up to $3 \mathrm{~ms}$ in the worst case, allowing for a kinematic update frequency above $300 \mathrm{~Hz}$. Longer calculation times up to $3 \mathrm{~ms}$ are required when $\mathcal{Q}_{n s}$ does not intersect the hyper-plane $q_{2}=$ 0 , because the integration of the self-motion-manifold (see Eq. (3.20)) cannot be terminated prematurely. The largest timing bottleneck in this setup is the used data-communication method between PC and microcontroller and not in the practical implementation of the proposed IK method in this work.

The results show that the method is applicable in real-time applications. For full scale exoskeletons the method would be implemented in dedicated realtime software. This transition would allow for shorter calculation times and a faster communication with low-level joint controllers, allowing for more updates of the joint angle reference trajectories.

\subsection{DISCUSSION AND CONCLUSION}

In this chapter we have studied the kinematic design and a new differential IK algorithm for a $4 \mathrm{R}$ exoskeleton shoulder joint. We ensure convergence to a global (in the sense of repeating space) minimum on the nullspace configuration sets to avoid body collisions and have proper exoskeleton conditioning. By identifying a preferred, but not enforced, configuration (the attractor), we have outlined a method to always converge on the nullspace configuration set through linear or bang-bang negative feedback to a configuration closest (in an Euclidian sense) in jointspace.

Results show that this exoskeleton, combined with the algorithm, outperforms standard $3 \mathrm{R}$ joints in terms of conditioning. The joint velocities of the $4 \mathrm{R}$ are similar to $3 \mathrm{R}$ in an absolute average sense. Still, 3R designs demanded infinite velocities within the used benchmark motion. Also, collisions with the body and arm were fully avoided in the $4 \mathrm{R}$ case, but could not be tested for the $3 \mathrm{R}$ cases.

If the method has to be applied in the acceleration domain, one has to use higher order integration methods (such as Runge-Kutta) to avoid large numerical drift when traversing $\mathcal{Q}_{n s}$. This adds an extra layer of complexity we have now avoided. Furthermore, there would be the need for critically damped proportional and derivative negative feedback. 
The presented method is limited by the fact that solutions are found in the velocity domain instead of the acceleration domain. This may result in nonsmooth velocity profiles, imposing possibly infinite joint accelerations. In practice, this is a minor problem. Because admittance dynamics models are first order from force to velocities, the joint velocities are mostly always smooth.

Further limitation of any $4 \mathrm{R}$ shoulder exoskeleton, regardless of the control method and inverse kinematics method used, is the inherent safety problem considering the lack of mechanical joint limits. This problem is also present in several 3R exoskeletons, albeit to a lesser extent. Software imposed joint limits can be used, but these are inherently less safe than mechanical end-stops. 

Part II

HAPTICS \& CONTROL:

ADMITTANCE CONTROL 

Arvid Q.L. Keemink, Herman van der Kooij and Arno H.A. Stienen

You push with the heel of your hand; the suit feels it, amplifies it, pushes with you to take the pressure off the receptors that gave the order to push. [...] The suit has feedback which causes it to match any motion you make, exactly - but with great force. Controlled force... force controlled without your having to think about it.

— Robert Heinlein, Starship Troopers, 1959

Abstract-This chapter presents an overview of admittance control as a method of physical interaction control between machines and humans. We present an admittance controller framework and elaborate control scheme that can be used for controller design and development. Within this framework, we analyze the influence of feed-forward control, post-sensor inertia compensation, force signal filtering, additional phase lead on the motion reference, internal robot flexibility, which also relates to series elastic control, motion loop bandwidth and the addition of virtual damping on the stability, passivity and performance of minimal inertia rendering admittance control. We present seven design guidelines for achieving high performance admittance controlled devices that can render low inertia, while aspiring coupled stability and proper disturbance rejection.

LIST OF USED SYMBOLS AND ABBREVIATIONS

\begin{tabular}{ll}
\hline Symbol & Explanation \\
\hline$a$ & $\begin{array}{l}\text { subscript: relating to apparent dynamics transfer function or accel- } \\
\text { eration } \\
\text { subscript: relating to apparent dynamic property }\end{array}$ \\
$\begin{array}{l}\text { This chapter is accepted to be published in the International Journal of Robotics Research } \\
\text { (IJRR). }\end{array}$
\end{tabular}


physical or virtual generalized damping coefficient subscript: relating to the controller controller transfer function subscript: relating to the desired tracked trajectory transfer function denominator subscript: relating to external input force subscript: relating to force subscript: relating to feedback control subscript: relating to feed-forward control physical or virtual mechanical force or torque subscript: relating to force gain controller gain subscript: relating to a human's mechanical property

electrical current

subscript: relating to electrical current, or integral gain intrinsic dynamical property of the human limb imaginary part of the argument imaginary constant $\sqrt{-1}$ physical or virtual mechanical stiffness, controller gain or transmission ratio controller gain amplified by transmission ratio physical or virtual mechanical generalized inertia subscript: relating to the actuator (motor) Butterworth filter polynomial order transfer function numerator subscript: post-sensor effect subscript: relating to robot or gearing ratio set of all real numbers real part of the argument laplace variable subscript: relating to sampling time or structural stiffness Discrete time sampler 
sensor or state/signal estimator transfer function

subscript: relating to target dynamics

delay time

subscript: relating to torque

physical or virtual mechanical velocity or angular velocity

subscript: relating to the virtual dynamics parameters, virtual dynamics transfer functions or velocity

mechanical admittance, either virtual or physical

baseline system admittance

mechanical admittance that is guaranteed to be passive

mechanical impedance, physical

mechanical impedance, model

feed-forward mechanical linear or rotational damping

mass fraction between 0 and 1

feed-forward mechanical linear mass or rotational inertia

first order dynamical system time constant

angular frequency

\begin{tabular}{ll}
\hline Abbrev. & Explanation \\
\hline $4 C$ & four channel \\
CVT & continuous variable transformer \\
DA & digital-to-analog \\
DOF & degree of freedom \\
ez-width & environment z-width \\
LTI & linear time invariant \\
NAC & natural admittance control \\
PD & proportional-derivative \\
PDD & proportional-derivative-double-derivative \\
pHRI & physical human robot interaction \\
PI & proportional-integral \\
PID & proportional-integral-derivative \\
ZOH & zero-order-hold \\
\hline
\end{tabular}




\subsection{INTRODUCTION}

During physical human-robot interaction (pHRI) a robot measures motions of or forces from the human and adequately responds to these. Several control methods exist for controlling robots in contact with a mechanical environment [59], namely: (in)direct force control [60], impedance control [61], admittance control $[62,63]$ and full-state interaction control $[64,65]$. The human user is usually seen as a special case of the environment.

In this chapter we discuss the admittance control paradigm, a control method that is not commonly used for haptic interaction control [66]. By measuring the interaction force with the human user, the set-point to a low-level motioncontroller is changed through virtual model dynamics to achieve some preferred interaction responsive behavior [62, 60,67] (see Fig. 4.1). The motion controller is commonly a reference following velocity controller, due to power conjugation of force and velocity, and this is what we will assume in the remainder of the text.

By making the relation between the measured force and the velocity-reference, the virtual model dynamics, consistent with laws of mechanics, simulation of physical dynamical systems is possible [68, 69].

Admittance control is the opposite, or dual $[68,67]$, of impedance control [61]. During impedance control forces are applied, either through open loop or closed loop control, to the human user after device motion is measured. The naming reflects the causality of the used virtual model dynamics. Due to this dual nature of admittance control and impedance control, they naturally excel at both different ends of the 'haptic spectrum' $[70,68,66,71]$. For admittance controlled devices it is easier to render stiff virtual surfaces and a challenge to render low inertia. It is troubled by dynamically interacting with stiff real surfaces (constrained motion) [72, 73, 68]. Impedance control, on the other hand, is a better candidate to render low inertia but not to render stiff virtual surfaces. It is troubled by dynamically interacting with low inertia (free motion) [68].

\subsection{Motivation}

Although admittance control has been successfully applied in multiple devices (see Sec. 4.3.2), an overview of applications, properties and possibilities of admittance control is lacking. We provide an overview of the development and applications of admittance control. In addition, we briefly recapitulate the no- 


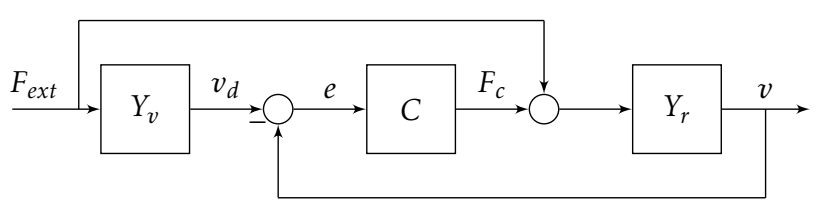

Figure 4.1: Basic stand-alone admittance control diagram of an uncoupled admittance controlled robot. It shows the measured externally applied force $F_{\text {ext }}$, passing through the virtual dynamics $Y_{v}$ to generate velocity reference $v_{d}$. A controller $C$ attempts to enforce this velocity on the robot $Y_{r}$ by applying a control force $F_{c}$ through an actuator (not shown). External force $F_{\text {ext }}$ also acts directly on the robot dynamics $Y_{r}$. The resulting motion of the robot is given by $v$.

tions of stability and passivity of admittance controlled systems. The main contribution of this work is the presentation of an elaborate admittance controller framework and its control scheme that summarizes major contributions from literature and experience, which can be used for controller design and development. Within this framework, we analyze the influence of 1) feed-forward control, 2) force signal filtering, 3) post-sensor inertia compensation, 4) the addition of virtual damping, 5) additional phase lead on the motion reference, 6) motion loop bandwidth, and 7) internal robot flexibility (which in the limit directly relates to series elastic control) on the stability, passivity and performance of minimal inertia rendering admittance control. Finally, these analyses lead to a set of design guidelines for achieving high performance admittance controlled devices that can render low inertia, aspiring robust coupled stability. The analyses are focus solely on single-DOF, single interface linear time invariant (LTI) systems with one-port admittance interaction only.

\subsection{BACKGROUND}

\subsubsection{Naming}

The name admittance control dates back to 1992 due to the developments of Newman and Gullapalli et al. [63, 74] and Schimmels and Peshkin [75]. Different names for what is commonly called admittance control can be found in literature: position based or velocity based impedance control $[76,77,78,79,80,81]$ and [82] respectively. It is sometimes interchanged with impedance control [83, 84]. 
In all cases there is the measurement of force that generates a motion control reference or a deviation from such a reference.

Some authors distinguish between motion-based impedance control and admittance control by focusing in the former case on motion tracking and in the latter case on force tracking $[85,59,86]$. We choose to use the generic term admittance control for all types of force-to-desired-motion relationships in this work, and recognize the fact that an admittance controller can track both motion and forces simultaneously.

The desired dynamical behavior, the admittance, felt at the 'interaction port' where the human interacts with the device, is called by different names: desired dynamics [87], target dynamics [87, 88], mechanical drive point mobility [63], virtual admittance/environment/model/dynamics [68, 67] or driving-point dynamics [89]. It could also be called the indirect force controller.

Dependent on the form of the desired dynamical behavior, several authors adopt different naming for the controller. The term admittance control is used for a inertia simulation [67], but also for pure damping [90,91] or generic force to motion simulation [70, 68]. Accommodation control is solely used for pure damping behavior $[62,63]$. Finally, compliance control is used for pure spring behavior [59]. If the controller is to mask only (static) friction effects and keep the same inertia (its natural admittance) as the robot system, Newman and Zhang [72] proposed the name Natural Admittance Control (NAC).

In this work, we take the aforementioned single analyses, and the major innovations and combine them into a single framework. We will use the term virtual dynamics (or virtual admittance) to describe the dynamics we want the device to display to the human, and to refer to the model that is used to calculate a velocity reference for a velocity controller to track. The dynamics that are actually felt by the human will be called the apparent dynamics (or apparent admittance), which preferably approaches the virtual dynamics.

\subsubsection{History and Applications}

Interaction control gained widespread academic interest after the pioneering work of Hogan [61] and Colgate [92] on impedance control and passivity at the end of the 1980s. The first mentions of using a control method very similar to admittance control date back to Whitney [62], where it was used to respond to hard contact in industrial applications and therefore for indirect force control purposes. 
Initially, interaction control was developed for applications such as welding and deburring, where stiff robot position control was highly impractical due to high stiffness and friction of the processed parts [62, 77, 75, 93, 85]. Accommodation and admittance control were first introduced on retrofitted industrial robots [62, 60, 77, 78, 94, 88]. Ott and Nakamura [81] exploit a force sensor in the base to increase safety of the system. Bascetta et al. [95] use variable admittance control for teaching of industrial manipulators to interact safely during manufacturing.

A patent from Fokker Control Systems (US4398889 A) describes admittance control in Flight Simulator devices in the field of control loading, starting from 1980. First mentions pHRI come from haptic master devices to render virtual dynamics in flight simulation and later in more generic scenarios [96, 97, 98]. In these cases virtual environments with admittance causality could be simulated, allowing more straightforward rendering of constrained motions.

Mentions of active devices capable of safe interaction between human and machines emerged at the beginning of the 1990s [99, 100]. Further development of the method led to successful practical admittance based devices such as the HapticMaster $[69,101]$ for generic haptic simulation, the Simodont for the training of dental practice, and Lopes II [102] for the rehabilitation of human walking, all developed by Moog Inc. [103].

Faulring et al. $[104,66]$ mention the use of Cobots with Continuous Variable Transformers (CVTs) to be able to render stiff constraints in an admittance control mode. Other methods employ admittance control in a master slave setup $[105,106]$ for surgery.

Exoskeleton control, used for the upper extremities [39, 107, 108, 109, 110], is sometimes implemented as multi-DOF admittance-controlled devices to aid in rehabilitation $[111,112,45,113,114,91]$. Rendering low inertia and task dependent stiffness assist the wearer in making motions with the arm. Due to the motion-controlled nature of the device, it can switch seamlessly between admittance control and pure motion-control. This makes it a good candidate for identification of the human neuromusculoskeletal system dynamics through applied position perturbations, and for switching between automated, reactive and cooperative tasks, as explained by Stienen et al. [115].

Several lower extremity exoskeleton devices use admittance control to render low impedance (high admittance) during the generation of locomotion patterns for rehabilitation purposes [102, 116]. For mobile lower-extremity rehabilitation the admittance controller is used to have carts move with the patient with 
minimal effort [117]. Other designs are developed for knee recovery specifically $[84,118]$. A method used by Aguirre-Ollinger et al. [84, 119] is to use admittance control with acceleration feedback as implicit force control to reduce the inertia of the lower leg of the human to facilitate knee recovery [13]. Rehabilitation of the ankle with admittance control is described by Saglia et al. [120].

Admittance control for end-point interaction is mainly used for power amplification or load reduction $[121,122,123,124]$ and the masking of unwanted dynamical effects in industrial applications. In these cases the heavy-load bearing capabilities of large and strong devices can result in substantial power amplification of a human user.

Special cases of admittance control can be found for interaction with humanoids [125, 126], anthropomorphic arms and hands [127], aerial vehicles [128] and mobile carts [129].

Furthermore, learning and adapting admittance control schemes have been implemented [74]. Adaptive models, time-varying parameters, or neural networks are used to optimize the interaction between the device and the human towards some objective $[130,131,132]$.

\subsubsection{Design Challenges}

Due to the velocity or position controlled nature of many admittance controlled devices, it is straightforward to create stiff or dissipative haptic constraints to assist in cooperative human-robot tasks. When the human is not supposed to be constrained, the device should have high admittance (i.e. low impedance). Preferably, the apparent admittance should be higher than the natural admittance of the inert, heavy and dissipative robot.

Infinite admittance, or zero impedance, over the complete frequency range is impossible to achieve on an admittance controlled device due to division by zero in the force-velocity relationship. A common approach is to have the virtual dynamics be a pure virtual inertia [84] that is as 'low as possible', while retaining stability when coupled to the user. The pure virtual inertia assures low impedance for low frequencies, attenuation of high frequencies and nondissipative behavior. The low virtual inertia admittance approach is the same as high integral indirect force control with an inner velocity-control loop. The integral force gain is the reciprocal of the virtual inertia. Effectively, the low virtual inertia generates a force controller that attempts to minimize the interaction force between device and the user. 
A problem with this method (further described in Sec. 4.5.3) is that when lowering the virtual inertia, the robot becomes unstable when in contact with stiffened human limbs or stiff environments. To reduce the apparent inertia while keeping safe and stable interaction behavior is therefore a challenge for admittance control.

Due to the high bandwidth of the inner motion-control loop, the admittance controller can achieve significant masking of nonlinear static friction effects inherent to the device itself [72]. The drawback of such a high motion-control bandwidth is the sensitivity of the controller to drive-train backlash and flexibility. Drive-train backlash and flexibility can result in unstable position-velocity limit-cycles [84].

\subsubsection{Admittance Control Placed in Perspective}

\section{Admittance control as a form of teleoperation}

Admittance control can be seen as a form of indirect force control [59], or as a specific case of a bilateral teleoperation controller. The latter fits the framework of the 4C Controller, as popularized by Lawrence [133] and HashtrudiZaad and Salcudean [134]. In this case it comprises a virtual admittance slave with possibly added virtual environment, without any communication delays. In this framework it is called the position-force architecture, reflecting the human causality instead of model causality. Attempting to simulate any 'virtual slave' system on an admittance controlled setup is similar to designing a masterslave setup with dissimilar master-slave dynamics and kinematics.

\section{Admittance vs. impedance control}

The main difference between admittance control and impedance control is that the former controls motion after a force is measured, and the latter controls force after motion or deviation from a set-point is measured [67].

Impedance controlled devices are commonly used for manual haptic and teleoperation displays. Admittance control is used more often in larger non-backdrivable high-friction devices that are of the full-body type (e.g. wearable robotics) and heavy-duty type (e.g. industry). This difference is mainly due to the ease of designing adequately performing impedance controlled devices with open loop force generation. It circumvents the need of using a force sensor, which is generally expensive and sensitive to drift and temperature change, and does not 
demand stiff mechanics of the robot as is preferred for a closed loop force controlled system. A drawback of such an impedance control method is the disturbing 'feel' of the remaining parasitic dynamics and friction effects of the device itself [68]. Therefore these impedance devices are commonly designed to be lightweight and to have low friction. If the impedance control force generation is open loop, the device is highly forgiving to backlash and drive train flexibility.

If explicit force control is used in the impedance controller, i.e. impedance control with force feedback $[68,87,66]$, the system's parasitic dynamics are highly suppressed. Nevertheless, low-frequency resonant modes and backlash will destabilize the system [68]. The closed loop control of force in impedance control, and the closed loop control of motion in admittance control, result in better approaching of the virtual dynamics. Possible noncollocation of force sensor and actuator limits the achievable force control bandwidth in impedance control. This is less of a problem in admittance control, since the actuator and velocity sensor are usually collocated, although such internal flexibility allows for less robust coupled stability and reduced approximation of the virtual dynamics. The range of achievable apparent dynamics or z-width [135] is higher for admittance control than for impedance control $[68,66]$.

\subsection{STABILITy AND PASSIVITY}

In contrast to a motion servo, a system that focuses on stable physical interaction aspires several kinds of stability [89], of which the last will be discussed separately;

1. Uncoupled stability, when the device is 'free', not being in contact with a human.

2. Contact transition stability, when transitioning from being free to being in contact.

3. Coupled stability, when the device is and stays in contact with a user or environment.

In practical cases the admittance controlled robot will make contact, or will already be in contact with a human, an object or the fixed world. The possible making or breaking of contact, is a contact transition, which can lead to nontrivial transition or switching instability [136]. We neglect the transitioning stage in our analyses, assuming a robotic device that has been held by, or attached 


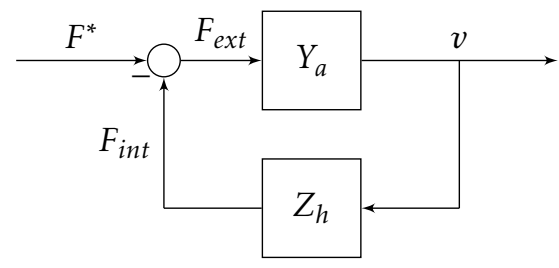

Figure 4.2: Interconnection of admittance controlled robot that has apparent admittance $Y_{a}$ and the human that has impedance $Z_{h}$, creating closed loop (coupled) interaction behavior. The external voluntary force from the human is given by $F^{*}$, the force generated by the intrinsic human dynamics is given by $F_{\text {int }}$, which both sum tot the total external force $F_{\text {ext }}$ experienced by the robot. This external force passes through the complete system dynamics $Y_{a}$ (see Fig. 4.1) and results in real velocity $v$.

to, a human user for sufficiently long time, or has its controller software started while already fully in contact or when fully uncoupled.

\subsubsection{Coupled Stability}

A human and machine being in contact, exchanging mechanical power or exerting forces bilaterally, behave as a single coupled system as shown in Fig. 4.2. Coupling stability is nontrivial, since two separately stable systems can exhibit coupled instability [92], or an unstable robot system could become stable after coupling it to a human user.

The coupling of an admittance controlled device with apparent admittance $Y_{a}$ to an impedance human user $Z_{h}$ creates a 'force loop' with negative feedback. For controlled devices interacting with the environment, the stability behavior is therefore highly dependent on the environment's impedance characteristics [59].

\subsubsection{Robust Coupled Stability: Energy Passivity}

The analysis method related to energy passivity [137] made its way from electrical network coupling-stability to robot-human and robot-environment interaction. It allows to use a similar argument to guarantee stability of robots during interaction (i.e. coupling) with all possible energetically passive environments. If a robot is designed to behave passively and consequently interacts with a hu- 
man, this guarantees (practical) robust coupled stability. This makes sense from the everyday experience where humans do not experience coupled instability when mechanically interacting with passive objects in the environment, even though we cannot guarantee that a human only exhibits purely passive behavior.

Therefore, energetically passive behavior of the apparent dynamics of the controller robot, together with good performance, form a design 'goal' to aim for, since it puts the responsibility of interaction stability with the human. Passivity conditions are therefore useful during controller design, and are investigated in the remainder of this work.

\section{Definition}

The definition of an energetically passive one-port system is that it cannot deliver more energy than what was put into it [92]; i.e for mechanical systems it would be required that

$$
\int_{-\infty}^{t} F(\tau) v(\tau) d \tau \geq 0
$$

where $F$ and $v$ are power-conjugated force and velocity inputs or outputs of a mechanical system of either admittance or impedance causality. If the apparent dynamical behavior of the robot during free motions is designed to behave like a passive system in accordance with (4.1), stability is guaranteed for any combination of the passive robot coupled to another passive system.

Colgate [92] described a method to assess passivity in the frequency domain for LTI systems. A single-DOF LTI controlled robot, in our case the uncoupled apparent dynamics $Y_{a}$ in admittance form (see $Y_{a}$ in Fig. 4.2) is passive if and only if;

(a) $Y_{a}(s)$ has no poles in the right half of the complex plane (i.e. uncoupled stability)

(b) Any imaginary poles of $Y_{a}(s)$ are simple and have with positive real residues (i.e. a positive coefficient after partial fractional expansion)

(c) $\mathfrak{R}\left\{Y_{a}(j \omega)\right\} \geq 0, \forall \omega \in \mathbb{R}$ (i.e. the admittance is positive real for all positive and negative real frequencies. For discrete time systems this is required up to the positive and negative Nyquist frequency). 
The first condition we usually conform to in stable motion control. The combination of the second and third conditions is commonly referred to as the positive real condition [92], which provides useful design guidelines. Following Dohring and Newman [88], the positive real condition for systems without time-delay reduces to the demand that $\mathcal{K}\{N\} \mathfrak{K}\{D\}+I\{N\} I\{D\} \geq 0, \forall \omega \in \mathbb{R}$, with $N$ and $D$ being the numerator and denominator of $Y_{a}$ respectively, and $\mathcal{R}\{\cdot\}$ and $I\{\cdot\}$ the real and imaginary parts of the argument, respectively. This condition leads to an even polynomial in angular frequency $\omega$. If the coefficient for the $\omega^{0}$ term is zero, all remaining polynomial coefficients should be greater than, or equal to, zero to have a passive dynamical system. These coefficients being greater than, or equal to, zero, including the uncoupled stability conditions, give all the necessary passivity conditions. If the polynomial has a non-zero coefficient for $\omega^{0}$, then then finding marginal passivity conditions can get more involved. Fourth order polynomials, which are effectively second order polynomials in $\omega^{2}$, then require discriminant analysis. For higher order polynomials there might not be a generally applicable method to find analytical marginal passivity conditions. Nevertheless, the more straightforward demand that all coefficients should be greater than, or equal to, zero for a polynomial in $\omega$ of any order guarantees a passive system, albeit conservative (i.e. dissipating). In the analyses in this work we will use this more strict demand that all polynomial coefficients should be greater than, or equal to, zero to determine system passivity.

A consequence of the positive real condition is that, the apparent dynamics $Y_{a}$ cannot have a relative pole-zero excess greater than 1 and the system has to be minimum-phase (i.e. no unstable zeroes).

\section{Practicality:}

Several authors suggest that enforcing passivity is too conservative for humanmachine interaction $[68,134,138,139,140]$. This is mainly due to the fact that the human interaction impedance in practice is bounded. Therefore aiming for coupled stability with any human limb that can be infinitely stiff, infinite in inertial mass, or infinitely dissipative, is conservative.

A design method used by Adams and Hannaford [68] to take finite human impedance into account, is to absorb the maximal and minimal human admittance into the robot's apparent admittance. The new robot admittance is coupled to an abstract passive human impedance that is allowed to take on any value. This allows for application of the positive real condition for design, while still accounting for the limited human impedance range. 
Investigations into the limited impedance ranges of the human arm are also discussed by Buerger and Hogan [138]. The coupled stability problem is consequently handled as a robust control problem with known parametric uncertainty in the human impedance parameters.

Haddadi [140] developed a passivity-based robust stability method which is less conservative than the aforementioned. Rules and visual aids are developed to incorporate bounds of the human or environment impedance for less conservative guaranteed stability conditions, with a better trade-off between stability and performance.

\subsubsection{EZ-width}

If the apparent dynamics $Y_{a}$ are not passive, then the coupled human-robot system in Fig. 4.2 is stable for a limited range of passive human behavior, parameterized by quasi-linear limb stiffness, damping and inertia values. When considering human stiffness and damping values only, this range is similar to the $\mathrm{z}$-width metric [135]. Instead of the dynamical parameters for which the robot is passive, our human stiffness and damping range describes the impedance of the human $\left(Z_{h}\right)$ (or environment) for which the coupled system is still stable. Therefore, we will call this allowable range of stiffness and damping: environment $\mathrm{z}$ width, or ez-width. The ez-width describes in what range a human's stiffness and damping can be for a system to be marginally stable for a human's limb inertia or another parameter. The ez-width can be calculated by evaluating the Routh array or Hurwitz determinants of the closed loop system from Fig. 4.2, namely $Y_{a} /\left(1+Z_{h} Y_{a}\right)$, or by evaluating the Nyquist criterion of the loop gain formed by $Z_{h} Y_{a}$. The ez-width diagrams in this work were calculated numerically, determining the phase margin of $Z_{h} Y_{a}$ for a passive $Z_{h}$ of the form $m_{h} s+b_{h}+\frac{k_{h}}{s}$. If the phase margin was negative, the coupled system was unstable. The ez-width diagrams show the demarcation between stable and unstable regions. The ez-width can be infinite. A robot with that property is energetically passive.

It should be noted that the usefulness of ez-width diagrams relies heavily on the major assumption that a second order passive quasi-linear mass-springdamper model is sufficient to describe neural feedback-controlled human limb behavior. Although several studies show that for certain tasks this assumption holds, e.g. [99], for other tasks or robot admittance it does not [141]. Therefore the ez-width diagrams only show best-case interaction scenarios where the hu- 


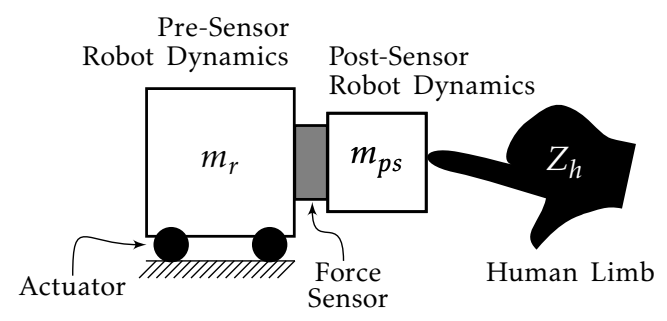

Figure 4.3: Generic electromechanical system overview of an admittance controlled device. An actuator moves all mechanics (robot inertia $m_{r}$ and some dissipation) placed before (i.e. 'pre') the force sensor. Behind the force sensor (i.e. 'post') there will be mechanics that generate force sensor measurements during motion $\left(m_{p s}\right)$. These post sensor mechanics consequently interact with the human limb $Z_{h}$. The sensor is assumed to be infinitely stiff and its mass is absorbed in $m_{p s}$.

man would behave fully passively. This assumption could be violated during more realistic real-world tasks, resulting in reduced effective ez-width.

\subsection{ADMITTANCE CONTROL MODEL}

In this section, a generic electromechanical set-up and a control model are presented to explain several of the observed instability and performance effects. The control model incorporates ideas from literature and from our experience. The goal of this section is to give the reader an introduction to a naive admittance controller design to expand upon with the 'guidelines' discussed in Sec. 4.6.

\subsubsection{Physical Setup}

A schematic admittance controlled device is shown in Fig. 4.3. An actuator generates mechanical power by the supply of electrical power through a controlled current or applied voltage. Such an actuator is commonly an electromechanical motor, although hydraulic actuation has been implemented successfully [80]. These actuators usually impose forces on the mechanics of the device, which consists of a drive train, moving parts and robotic links. Close to the interaction point' a force sensor measures the interaction forces with the user. This sensor is usually noncollocated with the actuator. 


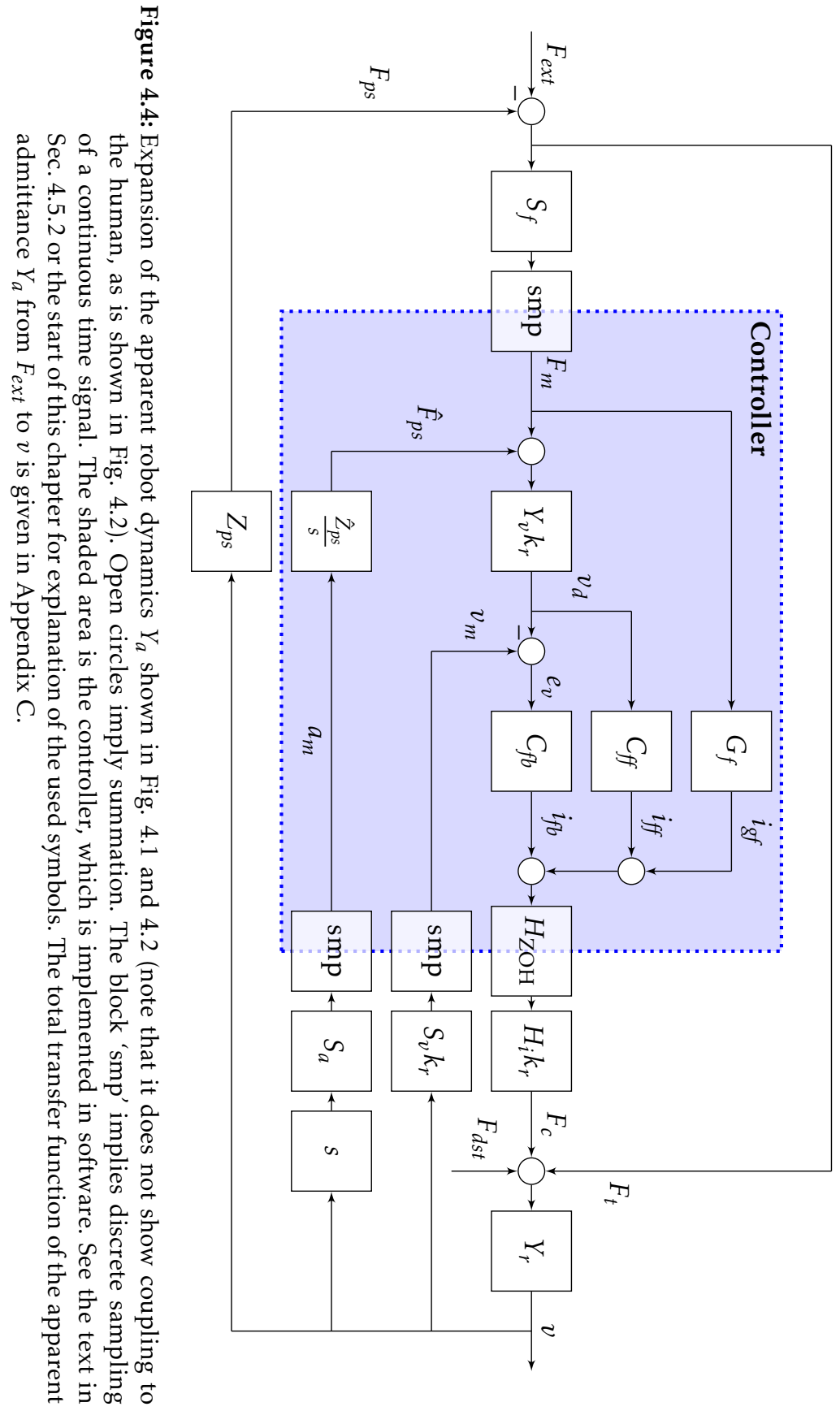


A force sensor has non-zero inertia, and usually a tool (for industrial applications), handle (for manual interaction) or cuff (for exoskeleton-like applications) is attached to the sensor. It will measure these post-sensor dynamics during motion of the pre-sensor system as an impedance effect. These post-sensor dynamics can be thought of as the known time-invariant impedance of the interaction environment, and is preferably solely inertial in nature. These postsensor dynamics do not include the user's dynamics. We therefore deem the user's impedance to be the unknown impedance of the environment, $Z_{h}$. Instead of the force sensor, the post-sensor dynamics interact with a human limb or another object in the environment. The consequential interaction force is measured by the force sensor. The admittance controller will, due to these forces, attempt to respond like the virtual dynamics.

\subsubsection{Admittance Control Diagram}

The stand-alone apparent dynamics $Y_{a}$ from Fig. 4.2 is shown in extended and expanded form in Fig. 4.4, omitting the interconnection with the user $\left(Z_{h}\right)$. The admittance causality is observed when noting the external force $\left(F_{\text {ext }}\right)$ as the input, tracking the signal to the motion (velocity) of the system $(v)$ as the output. The complete transfer function from force $F_{e x t}$ to motion $v$, describing this system's apparent dynamics, is given in Appendix C.

The total control diagram is comprised of several subsystems that will be discussed in the following paragraphs. Dependence on Laplace variable $s$ is mostly omitted for readability.

\section{Forces on the system:}

Externally applied force $\left(F_{e x t}\right)$ by the human and his/her passive dynamics, and forces from the post-sensor dynamics $\left(F_{p s}\right)$ act on this system. They are measured by a force sensor with limited bandwidth, possible filtering, or observer dynamics $\left(S_{f}\right)$.

\section{From measured force to desired velocity:}

The signal is consequently sampled (smp) to be processed by the digital controller. The measured forces $\left(F_{m}\right)$ pass through the virtual dynamics $\left(Y_{v}\right)$, which calculates the desired dynamical behavior. A transmission ratio $\left(k_{r}\right)$ increases the reference velocity of the virtual dynamics to the desired robot motor velocity 
$\left(v_{d}\right)$. This velocity, which is not necessarily a state from the virtual admittance, see section 4.6 .5 , is the reference signal for the velocity controller to track.

\section{Control and actuation:}

The velocity controller outputs a desired electrical current to be imposed on the actuators by the current controller. The velocity controller consists of a feedforward $\left(C_{f f}\right)$ and feedback controller $\left(C_{f b}\right)$. The feedback controller is commonly of the PI type: $C_{f b}=k_{p}+k_{i} / s$. Additional force amplification $\left(G_{f}\right)$ allows for apparent reduction in robot inertia and damping/friction effects.

All reference current values from the force-amplification, feed-forward and feedback control $\left(i_{g f}+i_{f f}+i_{f b}\right)$ are presented to the closed-loop current controller $\left(H_{i}\right)$. The output value is held constant during a sample time $T_{s}$ using a zero order hold $(\mathrm{ZOH})$. We assume the current controller to have high bandwidth (commonly $>2 \mathrm{kHz}$ for industrial current controllers), and some processing delay that adds to the sampling-and-processing delays from the $\mathrm{ZOH}$.

The controlled current generates a motor control force $\left(F_{c}\right)$ that is amplified by the gearing ratio $k_{r}$. This control force acts on the passive robot dynamics $\left(Y_{r}\right)$. External forces and disturbance forces $\left(F_{d s t}\right)$, such as static friction and obstructions also act on the robot and actuator.

\section{Resulting motion and impedance effects:}

The robot's resulting motion is due to the sum of these forces. This motion is measured by a velocity sensor or observer $\left(S_{v}\right)$, and an acceleration sensor or observer $\left(S_{a}\right)$. The former is used in the closed loop velocity control. The latter is used in compensation strategies (see Sec. 4.6.3) through $\hat{Z}_{p s}$. Any post-sensor dynamics $\left(Z_{p s}\right.$, the known environment impedance), i.e. a tool or cuff, generates impedance reaction forces $F_{p s}$ on the device's force sensor and adds to the robot dynamics directly through the forward path to $Y_{r}$.

\subsubsection{Control Model}

We are interested in a simple model that can explain instability when in contact with stiff environments. We call this model the baseline model, with which we can compare performance of possible improvements. It constitutes a naive admittance controller with feedback control only and virtual dynamics as in Fig. 4.1. The robot constitutes a rigid body mass with some dissipation, and is 


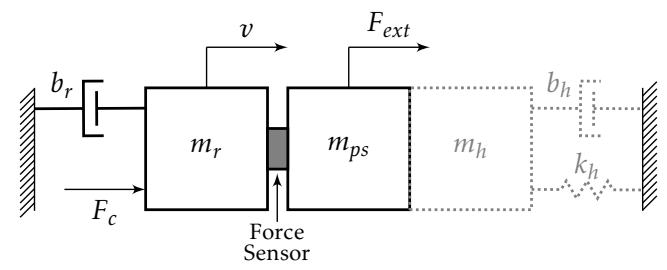

Figure 4.5: Schematic view of a rigid robot. An external force $F_{\text {ext }}$ and a controller force from an actuator $F_{c}$ are applied to the robot inertia $m_{r}$ combined with the post-sensor inertia $m_{p s}$, both resulting in some robot velocity $v$. Some energylosses during robot motion are modeled as viscous damping $b_{r}$. The robot can be rigidly connected to a human with inertia $m_{h}$, stiffness $k_{h}$ and damping $b_{h}$, shown by the gray dotted outline.

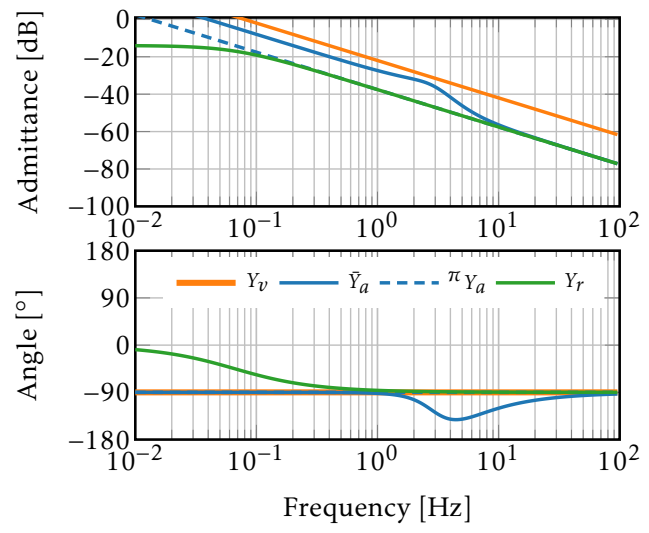

(a)

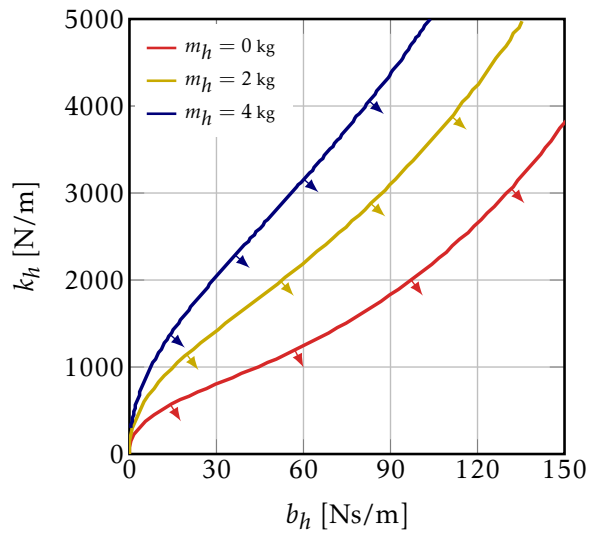

(b)

Figure 4.6: Behavior and performance of a typical admittance controlled system. (a) Bode plot of the uncoupled system: apparent dynamics $\bar{Y}_{a}$ approaches virtual dynamics $Y_{v}$ for low frequencies, but the constant difference is due to sensor inertia $m_{p s}$. Passive system ${ }^{\pi} Y_{a}$ has controller gains such that they conform to (4.7) and (4.8). This passive system performs poorly, very similar to the robot dynamics $Y_{r}$ instead of the virtual dynamics $Y_{v}$. (b) The ez-width of $\bar{Y}_{a}$ coupled to a second order impedance is larger for higher human limb inertia $m_{h}$. The region of stable interaction is indicated by the arrow markings. 
Table 4.1: Baseline system dynamical parameters

\begin{tabular}{ll}
\hline Parameter & Value \\
\hline$m_{v}$ & $2 \mathrm{~kg}$ \\
$k_{r}$ & 1 \\
$m_{r}$ & $10 \mathrm{~kg}$ \\
$m_{p s}$ & $2 \mathrm{~kg}$ \\
$b_{r}$ & $5 \mathrm{Ns} / \mathrm{m}$ \\
$k_{p}$ & $100 \mathrm{Ns} / \mathrm{m}$ \\
$k_{i}$ & $2000 \mathrm{~N} / \mathrm{m}$ \\
\hline
\end{tabular}

shown in Fig. 4.5. The apparent dynamics of this baseline system is denoted by $\bar{Y}_{a}$. This robot can be in contact with a human that applies force $F_{\text {ext }}$, which can be from human impedance (shown in dotted gray in Fig. 4.5).

This baseline model is derived from the elaborate model in Fig. 4.4. We assume ideal sensors, such that $\left(S_{f}=S_{v}=1\right)$, no acceleration sensing $\left(S_{a}=0\right)$, no feed-forward control $\left(G_{f}=C_{f f}=0\right)$, assume post-sensor impedance $Z_{p s}=m_{p s} s$, and set $m_{r}=m_{r}^{\prime}+m_{m} k_{r}^{2}$ and $b_{r}=b_{r}^{\prime}+b_{m} k_{r}^{2}$ to add the effects of reflected inertia $\left(m_{m} k_{r}^{2}\right)$ and damping $\left(b_{m} k_{r}^{2}\right)$ from the motor to those of the robot inertia $\left(m_{r}^{\prime}\right)$ and damping $\left(b_{r}^{\prime}\right)$. The used parameter values are presented in Table 4.1. The controller attempts to make a $10 \mathrm{~kg}$ inertia robot with damping feel like a pure $2 \mathrm{~kg}$ inertia, which gives an inertia reduction factor of five and removes damping effects.

The equation of motion of the system in Fig. 4.5, omitting the human impedance, absorbing any external force (either from human impedance or extraneous force) into $F_{\text {ext }}(t)$ is given by:

$$
\left(m_{r}+m_{p s}\right) \dot{v}(t)+b_{r} v(t)=F_{e x t}(t)+F_{c}(t)+k_{r} F_{d s t}(t),
$$

with $m_{r}$ the pre-sensor robot inertia and $m_{p s}$ the post-sensor robot inertia, $v(t)$ the real robot velocity, $b_{r}$ the viscous effects in the drive train, $k_{r}$ the transmission ratio of the drive train, $F_{\text {ext }}(t)$ the external force applied by the user (directly felt by the robot actuators), $F_{c}(t)$ the force applied by the controller through actuators and transmission, and $F_{d s t}(t)$ disturbance forces acting on 
the robot on the actuator side. Equation (4.2) is rewritten in the Laplace-domain (omitting dependency on $s$ for readability) by

$$
\left(m_{r} s+b_{r}\right) v=F_{e x t}-m_{p s} v s+F_{c}+k_{r} F_{d s t} .
$$

The controller equations for this baseline model for virtual dynamics of inertial form (virtual inertia $m_{v}$ ) are given by

$$
\begin{aligned}
Y_{v} & =\frac{1}{m_{v} s} \\
v_{d} & =k_{r} Y_{v}\left(F_{\text {ext }}-m_{p s} v s\right) \\
F_{c} & =k_{r} \frac{k_{p} s+k_{i}}{s}\left(v_{d}-k_{r} v\right) .
\end{aligned}
$$

with $k_{p}$ and $k_{i}$ the proportional and integral controller gains respectively. Equation 4.4 gives the transfer function of the virtual dynamics. Equation (4.5) shows that the reference velocity is calculated from the measured interaction force, namely external force $F_{e x t}$ and the post-sensor inertial effects $-m_{p s} v s$. Equation (4.6) shows a typical PI velocity controller that generates a controller force based on the velocity error $e_{v}=v_{d}-k_{r} v$.

\section{Uncoupled stability:}

For positive choices for all parameters, the apparent dynamics created by (4.3) to (4.6) has three poles: one of zero from the purely inertial virtual dynamics $Y_{v}$, and two stable (possibly complex) poles from the PI-feedback controller. Therefore, the robot is stable when in free air, when it is not held by the human.

In Fig. 4.6a is shown that the baseline apparent admittance $\bar{Y}_{a}$ is stable, and partially approaches the virtual dynamics $Y_{v}$. For low frequencies there is a constant difference in admittance modulus, which is an inertia offset due the post sensor inertia $m_{p s}$. The naive admittance controller can therefore not remove any post-sensor inertia (see Sec. 4.6.3 how to achieve this). At high frequencies the apparent dynamics $\bar{Y}_{a}$ drop back to the robot dynamics $Y_{r}$, introducing excess phase lag in the frequency range of the transition. 
Passivity of the uncoupled apparent dynamics:

Passivity of this robot is guaranteed if and only if

$$
\begin{aligned}
m_{v} & >0 \\
m_{v} & \geq \frac{K_{p}}{K_{p}+b_{r}} m_{r} \approx m_{r} \\
-b_{r} K_{i} & \geq 0,
\end{aligned}
$$

with $K_{p}=k_{p} k_{r}^{2}$, and $K_{i}=k_{i} k_{r}^{2}$. Equation (4.8) tells us we have to sacrifice low frequency performance for passivity by setting $k_{i}=0$ (it cannot be made negative, since that would violate the uncoupled stability requirement). This is understandable from the fact that the integral controller adds extra phase lag for low frequencies onto the already marginally passive virtual inertia behavior. Therefore any amount of extra phase lag makes the apparent admittance active. At the passivity limit given by (4.7), which demands to have the controller introduced pole in $Y_{a}$ to be of higher frequency than the introduced zero we are left with a passive equivalent system with the same inertia as the robot itself (see Fig. 4.6a, system ${ }^{\pi} Y_{a}$ ). Therefore, passive inertia reduction is not possible with admittance control with a pure virtual inertia and solely using feedback control. Having high transmission ratio (i.e. $k_{r} \gg 1$ ) makes it more difficult for such a system to be passive, according to (4.7). The passivity criterion tells us to use little integral gain, and use low transmission ratio. This conflicts with good disturbance rejection and performance.

\section{Coupled stability:}

The uncoupled baseline system with parameters described in Table 4.1 is not passive and will have finite ez-width, when coupled to a passive environment, as is shown in Fig. 4.6b.

All the stability boundaries in Fig. $4.6 \mathrm{~b}$ have in common that they pass through the origin, for any human limb inertia. This shows that admittance controlled systems would never be stable for interaction with pure springs, or pure springmass combinations. This is something that is not observed in practice, because all realistic environments have some form of energy dissipation. The upward slope of all curves through the origin shows that adding limb damping yields a decent 'stiffness margin' and stable interaction. 

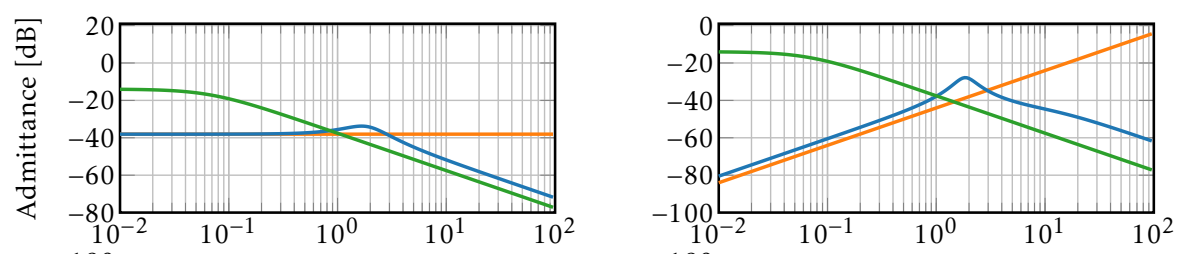

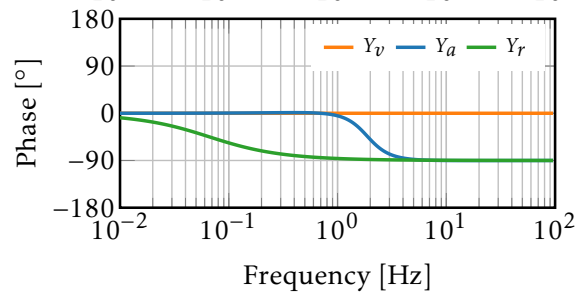

(a) Accommodation Control

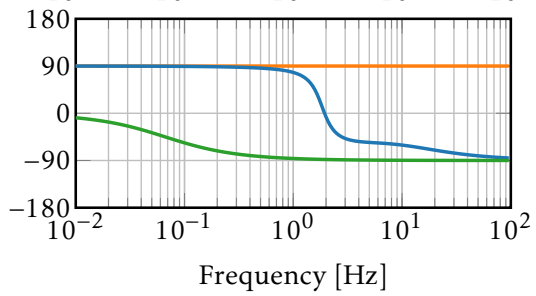

(b) Stiffness Control

Figure 4.7: Admittance control apparent dynamics $Y_{a}$ for uncoupled (a) passive accommodation control $\left(b_{v}=80 \mathrm{Ns} / \mathrm{m}\right)$, (b) passive stiffness control $\left(k_{v}=\right.$ $1000 \mathrm{~N} / \mathrm{m}$ ). Both figures share the same legend. The low frequency mismatch in (b) is due to the integral velocity gain $k_{i}$ that acts like a spring in series with virtual spring $k_{v}$. In both figures the phase of $Y_{a}$ stays within $\pm 90^{\circ}$, which shows passivity.

\subsubsection{Virtual Damping and Stiffness Behavior}

Naive admittance controllers can more straightforwardly render pure virtual damping (i.e. accommodation) and pure stiffness effects passively with decent performance. This is illustrated in Fig. 4.7a and Fig. 4.7b. For low frequencies the apparent admittance approaches the virtual admittance well for both accommodation and stiffness control. Above the feedback controller bandwidth, the apparent admittance becomes inertial in nature due to the robot's intrinsic dynamics.

If in (4.4) the virtual dynamics are replaced by $Y_{v}=\frac{1}{b_{v}}$ (i.e. accommodation form), the passivity conditions become

$$
\begin{aligned}
b_{v} & \geq 0 \\
m_{r} K_{i} & \leq\left(K_{p}+b_{r}\right)\left(K_{p}+b_{v}\right) .
\end{aligned}
$$


This shows again that $K_{i}$ should be kept low, the robot inertia has to be low and that either the virtual damping, robot damping or proportional gain has to be high to have passive accommodation control.

If in (4.4) the virtual dynamics are replaced by $Y_{v}=\frac{s}{k_{v}}$ (i.e. stiffness form), the relevant passivity condition is trivial with $k_{v} \geq 0$, when assuming positive values for all other parameters. The apparent stiffness $k_{\text {app }}$ of the device is

$$
k_{a p p}=\left(\lim _{s \rightarrow 0}\left(\frac{Y_{a}}{s}\right)\right)^{-1}=\left(\frac{1}{k_{v}}+\frac{1}{K_{i}}\right)^{-1}
$$

or two springs (the integral/position gain and the virtual stiffness) in series, as can be seen in Fig. 4.7b. The apparent stiffness differs slightly from the virtual stiffness due to finite integral controller gain $k_{i}$.

\subsubsection{Virtual Element Combinations}

For combinations of mass-spring-damper elements in the virtual dynamics, the passivity conditions become combinations of the conditions presented in the previous sections. This leads to upper and lower limits of robot and controller parameters that become difficult to interpret as design-guidelines in some cases. The effective behavior of these passivity conditions, and what they effectively teach us, is shown in Table 4.2. Note that the mass-damper combination is also discussed in more detail in Sec. 4.6.4.

As a rule of thumb it can be stated that if virtual mass is used, the condition in (4.7) is invariant to addition of other elements. Also, the conditions for a spring-damper combination add directly (therefore reducing the passivity of a pure spring), but the mass-spring combination acquires an extra addition to the passivity condition.

Table 4.2 also gives a coupled stability robustness ranking from 1 (the best) to 7 (the worst) showing for a fixed set of controller and robot parameters, which virtual admittance makes the robot 'most' passive.

Note that the virtual mass-spring-damper case is the only combination that also has a non-trivial uncoupled stability requirement related to an upper limit on $k_{i}$. All other parameter combinations achieve uncoupled stability due to positive parameters. The generic mass-spring-damper passivity, and stability, conditions are derived and shown in more detail in Appendix C. 


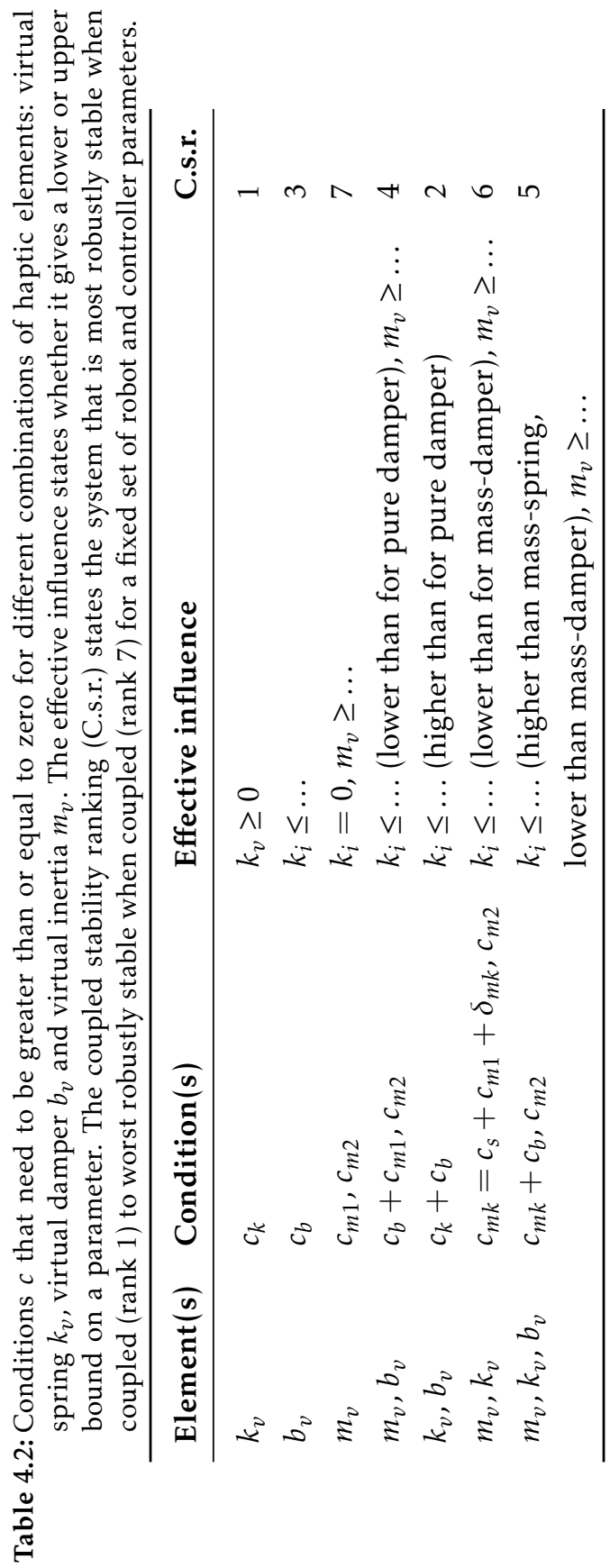




\subsection{GUIDELINES FOR MINIMAL INERTIA}

In section 4.5.4 it was shown that pure damping and stiffness are readily rendered passively by the robot. Therefore, we focus on the challenge of rendering low system inertia. We expand the naive model from Sec. 4.5.3 to incorporate and analyze additions to the control diagram that are shown in Fig. 4.4 and were discussed in Sec. 4.5.2. We use the passivity criterion for the uncoupled system, the ez-width of the system coupled to a passive second order system, disturbance rejection and admittance tracking performance (i.e. how well the apparent admittance matches the virtual admittance) to draw conclusions about the feasibility of certain design choices. We will always compare a change in design or model to the 'baseline' controller from Sec. 4.5.3, and attempt the same inertia reduction of a factor five from $10 \mathrm{~kg}$ to $2 \mathrm{~kg}$.

From this analysis follows a set of guidelines that is presented here in random order. The derivation of the apparent dynamical behavior, the uncoupled stability conditions and positive real conditions for all the guidelines are shown in Appendix C.

\subsubsection{Guideline 1: Use Feed-Forward Control}

If the robot controller can be used in torque (or current) control mode it is beneficial to use feed-forward control. Feed-forward control can be applied in the form of force gain $\left(G_{f}>0\right)$ and inertia and damping compensation (impedance $\left.C_{f f}=\mu_{f f} s+\beta_{f f}\right)$. The passivity condition in (4.7) and (4.8) change due to the addition of feed-forward control to

$$
\begin{aligned}
m_{v} & \geq \frac{\left(K_{p}+\beta_{f f} k_{r}^{2}\right) m_{r}-\left(K_{p}+b_{r}\right) \mu_{f f} k_{r}^{2}}{\left(G_{f} k_{r}+1\right)\left(K_{p}+b_{r}\right)} \\
0 & \leq\left(\beta_{f f} k_{r}^{2}-b_{r}\right) K_{i} .
\end{aligned}
$$

By setting $\beta_{f f} k_{r}^{2} \geq b_{r}$ in (4.10), it is possible to use integral gain for good low frequency approach of the apparent dynamics to the virtual dynamics. The feedforward inertia parameter $\mu_{f f}$ effectively removes inertia from the robot, such that there is less inertia to reduce by the feedback controller. This can be seen in the numerator of (4.9) where feed-forward inertia $\mu_{f f}$ is subtracted from the robot inertia $m_{r}$. The inertia-increasing effect of $\beta_{f f}$ on the right hand side of (4.9) can be counteracted by using $G_{f}>0$. 

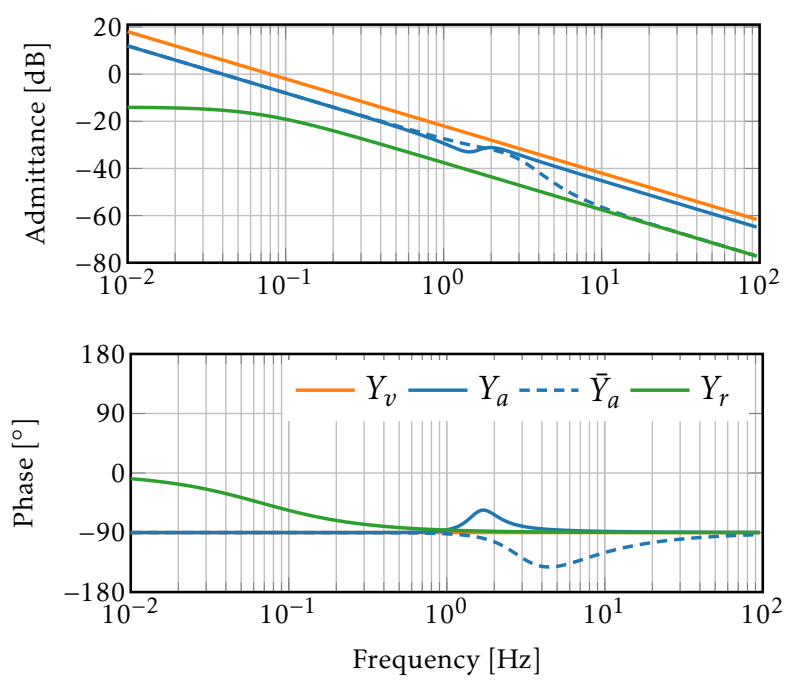

Figure 4.8: Comparing the use of feed-forward control $\left(Y_{a}\right)$ with the baseline system $\bar{Y}_{a}$. It can be seen that high frequency approach of the virtual dynamics is good for $Y_{a}$. Furthermore, the phase lag of $Y_{a}$ stays within passivity bounds, as opposed to $\bar{Y}_{a}$. Used parameter values: $G_{f}=5, \mu_{f f}=10 \mathrm{~kg}$ and $\beta_{f f}=2 \mathrm{Ns} / \mathrm{m}$.

For high transmission ratios, the passivity condition in (4.9) reduces to

$$
\mu_{f f} \geq \frac{k_{p}+\beta_{f f}}{k_{p}+b_{m}} m_{m} .
$$

This shows that only with feed-forward, high transmission actually helps in achieving some passive low virtual inertia.

The use of feed-forward increases both the ez-width and improves the admittance tracking performance for high frequencies above the velocity controller bandwidth. As is shown in Fig. 4.8, the admittance can be made passive (the ezwidth becomes infinite), while approaching the virtual admittance much better 


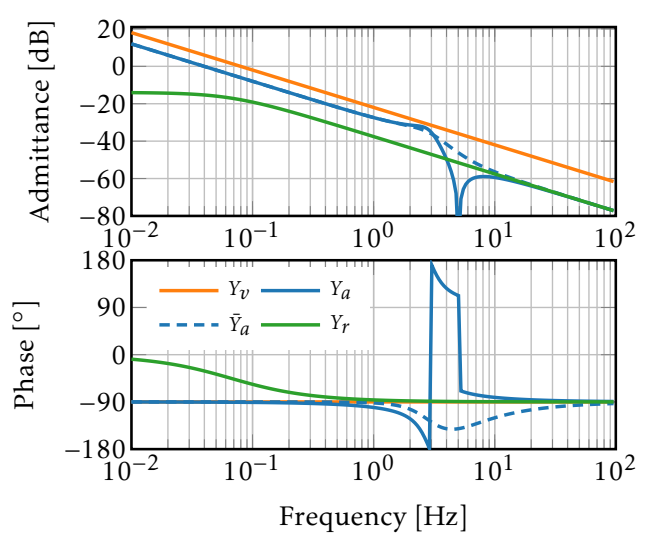

(a)

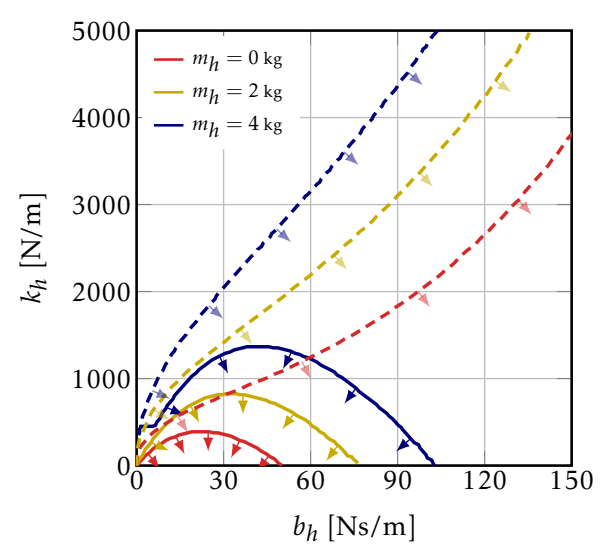

(b)

Figure 4.9: Influence of low-pass filtering the measured force on system performance and interaction stability. (a) This bode plot shows a system with force filtering $\left(Y_{a}\right)$ and the baseline system $\bar{Y}_{a}$. The used filter is of first order with high time constant $0.05 \mathrm{~s}$ to show an extreme effect on phase-lag and consequently on ez-width. It can be seen that high frequency approach of the virtual dynamics is poor for $Y_{a}$. Furthermore, the phase-lag of $Y_{a}$ is bigger than for $\bar{Y}_{a}$, resulting in a system much more unstable when in contact with a human limb. (b) The ez-width of $Y_{a}$, compared to baseline (dashed lines). Due to force filtering the ez-width is reduced. The region of stable interaction is indicated by the arrow markings.

at high frequencies than the baseline system $\bar{Y}_{a}$ could. The apparent inertia for high frequencies is given by

$$
\begin{aligned}
m_{a p p} & =\left(\lim _{s \rightarrow \infty}\left(s Y_{a}\right)\right)^{-1} \\
& =m_{p s}+\frac{m_{r}}{\frac{\mu_{f f}}{m_{v}} k_{r}^{2}+G_{f} k_{r}+1} .
\end{aligned}
$$

Without any feed-forward (i.e. $G_{f}=0$ or $\mu_{f f}=0$ ) the high frequency inertia would always fall back to the total robot inertia $m_{p s}+m_{r}$. The use of feedforward control passively reduces this inertia, but it cannot become lower than $m_{p s}$. 


\subsubsection{Guideline 2: Avoid Force Filtering}

It is tempting to low-pass filter force sensor measurements to reduce effects of noise or aliasing that cause random motion of the robot. This should be avoided if the virtual admittance is purely inertial (i.e. $Y_{v}=1 / m_{v} s$ ). Consider a force sensor bandwidth limitation given by

$$
S_{f}(s)=B_{n}^{-1}(s) \text {, }
$$

with $B_{n}(s)$ a Butterworth polynomial of order $n$. For all orders $n>0$ we add extra poles and $n \pi / 2$ rad phase-lag to the virtual admittance $Y_{v}$. This extra phase lag directly conflicts with the relative-order constraint from the frequency domain passivity criterion (see Sec. 4.4.2). A single pole low pass filter with time constant $\tau_{f}$ would change the passivity condition of (4.7), only if $k_{i}=0$ to:

$$
m_{v} \geq m_{r} \frac{K_{p}}{K_{p}+b_{r}}+K_{p} \tau_{f} .
$$

Setting $k_{i}$ and then picking $m_{v}$ on the passivity bounds would not lead to any decrease in inertia. Adding a low pass filter with $\tau_{f}>0$ makes this effect even worse, requiring an increase in virtual (and therefore apparent) inertia for the system to be passive.

Filtering will therefore reduce ez-width (see Fig. $4.9 \mathrm{~b}$ for an extreme case of low-pass filtering) and limit high frequency apparent admittance performance (see Fig. 4.9a). This is not problematic for $n=1$ with accommodation control, or $n=2$ for stiffness control, which will both effectively become admittance control due to the extra pole(s) of the filter (see Appendix C).

If filtering is inevitable, e.g. for anti-aliasing, then the filter bandwidth should be as high as possible and the filter order as low as possible.

\subsubsection{Guideline 3: Compensate Post-Sensor Inertia}

Post-sensor dynamical effects are not reduced or masked by the basic admittance controller (Sec. 4.5.3), or by feed-forward control (Sec. 4.6.1). The post-sensor inertial effects can be compensated in the low-frequency range by performing 


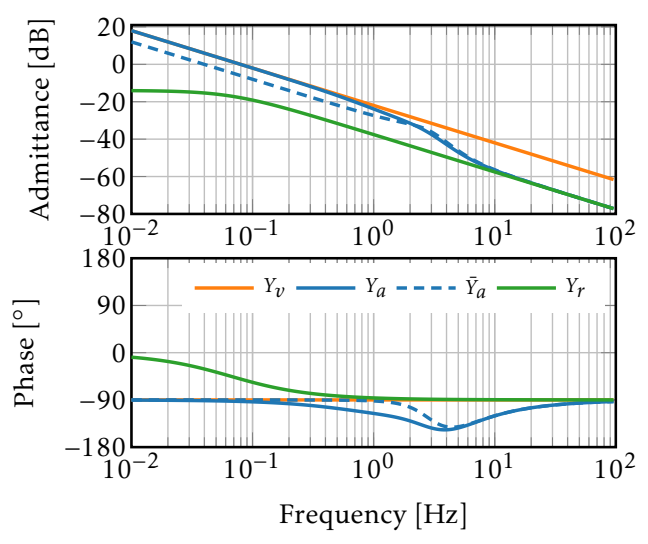

(a)

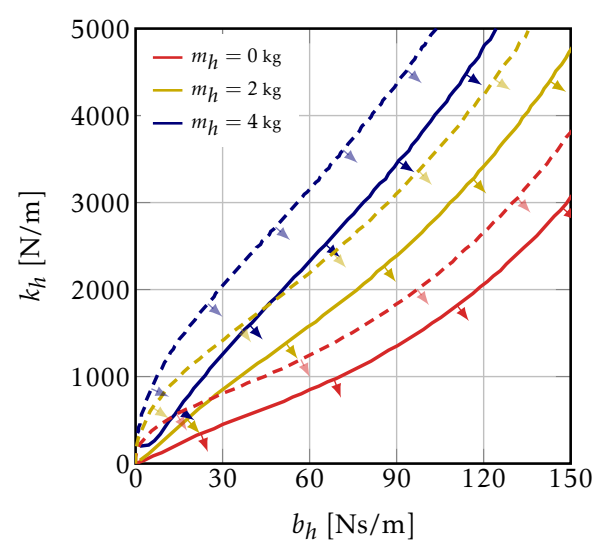

(b)

Figure 4.10: Influence of post-sensor compensation on system performance and interaction stability. (a) This bode plot shows a system with post-sensor compensation $\left(Y_{a}\right)$ and the baseline system $\bar{Y}_{a}$. The used amount of post-sensor inertia compensation was $\mu_{c}=2 \mathrm{~kg}$, the same amount as the post-sensor inertia, with low-pass filter $\tau_{c}=0.1 \mathrm{~s}$. The compensation improves low-frequency tracking, but generates phase lag. (b) Due to the added phase lag, the ezwidth of $Y_{a}$ (solid lines) is lower than that of $\bar{Y}_{a}$ (dashed lines). Post-sensor inertia compensation therefore reduces ez-width. The region of stable interaction is indicated by the arrow markings.

post-sensor dynamics compensation (in impedance form) with a compensation inertia $\mu_{c}$, and low pass filter time constant $\tau_{c}$ :

$$
\hat{Z}_{p s} S_{a}=\frac{\mu_{c}}{\tau_{c} s+1}
$$

This improves the performance, because indeed we achieve the following apparent inertial behavior at low frequencies:

$$
\begin{aligned}
Y_{a, \text { low }-\mathrm{freq}} & \approx \frac{1}{s} \lim _{s \rightarrow 0}\left(s Y_{r}\right) \\
& =\frac{1}{s\left(m_{v}+m_{p s}-\mu_{c}\right)}
\end{aligned}
$$

If $\mu_{c}=m_{p s}$ the post sensor dynamics are completely compensated, as shown in Fig. 4.10a. 
This method, however, reduces ez-width (see Fig. 4.10b). The passivity condition in (4.7) changes to (assuming $\tau_{c}=0$ )

$$
m_{v} \geq \frac{K_{p}}{K_{p}+b_{r}}\left(m_{r}+\mu_{c}\right),
$$

where $\mu_{c}$ effectively increased the lower bound on the value of $m_{v}$.

In accordance with with Aguirre-Ollinger et al. $[119,13]$ this method can also be used to effectively give the robot negative inertia. This will reduce the inertia of the object or human limb attached to the robot. For this to work, $\tau_{c}>0$ (or even higher order filters) and some limb damping $b_{h}>0$ is required.

\subsubsection{Guideline 4: Use Some Virtual Damping}

Virtual admittance of inertial form can in most applications be changed to a combination of inertia and a small amount of damping

$$
Y_{v}=\frac{1}{m_{v} s+b_{v}}
$$

The small amount of damping $\left(b_{v}\right)$ is hardly felt by the user, but adds useful phase lead at lower frequencies that can lead to passivity and increased ezwidth, if the phase lead is near the excessive phase lag. Therefore, added virtual damping is successful when the velocity controller bandwidth is low or has long delays.

The passivity conditions in (4.8) changes, when adding some virtual damping, to

$$
K_{i} \leq b_{v} \frac{\left(K_{p}+b_{v}\right)\left(K_{p}+b_{r}\right)}{b_{r} m_{v}+b_{v} m_{r}}
$$

Passivity condition in (4.7) is left unaltered, i.e. adding some virtual damping will not allow for lower $m_{v}$. A third new passivity condition is the rather trivial one $b_{v} K_{i}^{2} \geq 0$. Since integral gain can be increased, virtual damping allows for better low frequency tracking (see Fig. 4.11a at the low frequencies).

Fig. $4.11 \mathrm{~b}$ shows that ez-width becomes larger when adding some virtual damping. A minor penalty for using damping is the dissipative nature, impeding motion. 


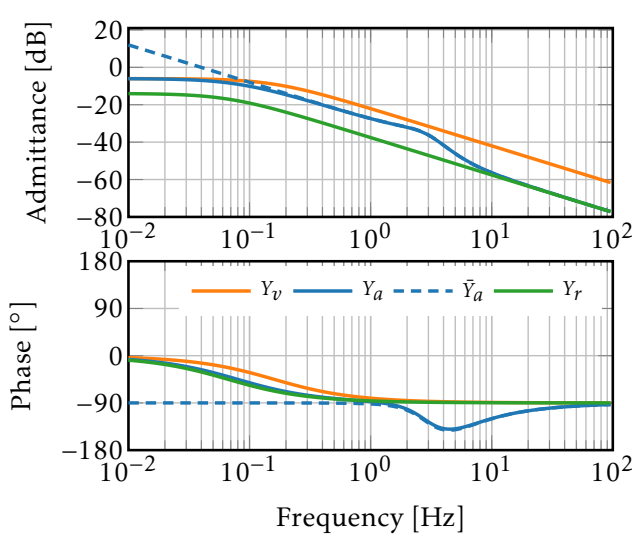

(a)

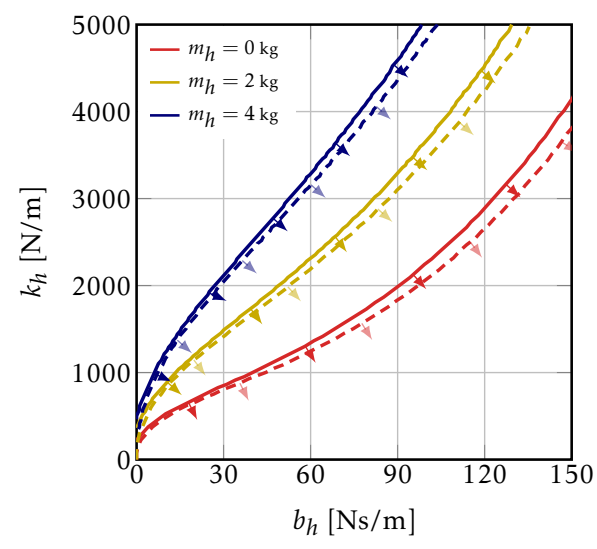

(b)

Figure 4.11: Influence of adding virtual damping on system performance and interaction stability. (a) This bode plot shows a system with some virtual damping $\left(Y_{a}\right)$ and our baseline system $\bar{Y}_{a}$. The added damping has a value of $b_{v}=2$ Ns/m. (b) The virtual damping reduces some phase lag. The ez-width of $Y_{a}$ (solid lines) is larger than that of $\bar{Y}_{a}$ (dashed lines). Using a small amount of damping therefore increases ez-width. The region of stable interaction is indicated by the arrow markings.

\subsubsection{Guideline 5: Modify the Velocity Reference}

It is common that industrial robots with 'black box' PI velocity control (or equivalently PD position control) are retrofitted with an admittance controller. In that case, adding feed-forward (guideline 1) is not possible, and some other way has to be found to get better admittance tracking and good ez-width.

It is possible to change the virtual admittance and add some form of acceleration feed-forward with gain $k_{a}$

$$
Y_{v}=\left(s k_{a}+1\right) Y_{v}^{\prime}
$$

with $Y_{v}^{\prime}$ some intended virtual dynamical behavior. This creates phase some lead, and better high frequency tracking of the originally intended virtual admittance $Y_{v}^{\prime}$. 

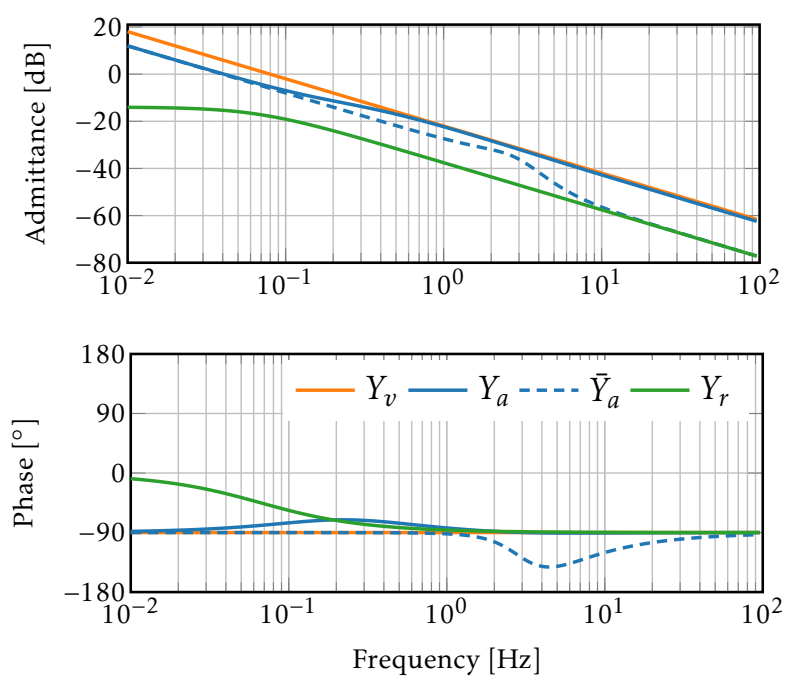

Figure 4.12: Influence of a system with extra phase lead $\left(Y_{a}\right)$ with our baseline system $\left(\bar{Y}_{a}\right)$ for $k_{a}=1$. The addition of phase lead improves admittance tracking, and reduces phase lag, resulting in a passive system.

The passivity conditions in (4.7) and (4.8) change to

$$
\begin{aligned}
m_{v} & \geq \frac{K_{p} m_{r}-k_{a}\left(K_{p}^{2}+K_{p} b_{r}-K_{i} m_{r}\right)}{K_{p}+b_{r}} \\
0 & \leq\left(k_{a} K_{i}-b_{r}\right) K_{i} .
\end{aligned}
$$

This complex looking condition gives us some advice: 1) use a robot with minimal inertia $\left.m_{r}, 2\right)$ keep integral velocity gain 'low' to benefit from $k_{a}$, although $K_{p}^{2}$ is usually so big this is not a problem. Also the addition of $k_{a}$ allows for passive use of integral gain. Therefore adding this additional phase lead will improve ez-width and performance (see Fig. 4.12). The use of high transmission ratio $k_{r}$ will reduce the condition in (4.12) to $k_{a} \geq 0$, ensuring passivity for any positive value of $k_{a}$. 


\subsubsection{Guideline 6: Increase Velocity Loop Bandwidth}

Many passivity conditions in the aforementioned guidelines demand low $k_{p}$ and low $k_{i}$ of the velocity controller. Nevertheless, high bandwidth control actually improves ez-width drastically. This seemingly contradicting statement comes from the fact that high bandwidth pushes the excessive phase lag to high frequencies, becoming only an issue for higher human stiffness values. Therefore increasing $k_{p}$ and $k_{i}$ could have beneficial effect on ez-width, while fully neglecting the passivity requirement. Furthermore, higher $k_{p}$ and $k_{i}$ values ensure more disturbance rejection at the motor side, which suppresses unwanted friction and parasitic dynamics.

Add differential velocity control:

An additional method to increase the velocity control bandwidth is to use a PID velocity ( $\mathrm{PDD}^{2}$ position) controller [142]. The feedback controller is augmented with differential gain $k_{d}$ and low pass filter time constant $\tau_{d}$, and it takes on the form

$$
C_{f b}=k_{p}+\frac{k_{i}}{s}+\frac{k_{d} s}{\tau_{d} s+1} .
$$

To be a proper and implementable transfer function, differentiation is bandlimited by the low pass filter. Unfortunately, the passivity condition from (4.8) remains unaltered. The passivity condition from (4.7) becomes:

$$
m_{v} \geq \frac{K_{p} m_{r}+b_{r} K_{i} \tau_{d}^{2}-b_{r} K_{d}}{K_{p}+b_{r}},
$$

with $K_{d}=k_{d} k_{r}^{2}$. This shows that the virtual inertia parameter has to be increased if there is non-zero $K_{i}$ and non-zero $\tau_{d}$. We also obtain a new condition, very similar to (4.7), which exists only if $\tau_{d} \neq 0$. It states that still no passive inertia reduction can be achieved

$$
m_{v} \geq m_{r} \frac{K_{p} \tau_{d}+K_{d}}{\left(K_{p}+b_{r}\right) \tau_{d}+K_{d}} \approx m_{r} .
$$

Band-limited differential control action has little effect on the passivity conditions, and it can not make the system passive with $K_{i} \neq 0$ and $\tau_{d} \neq 0$. 


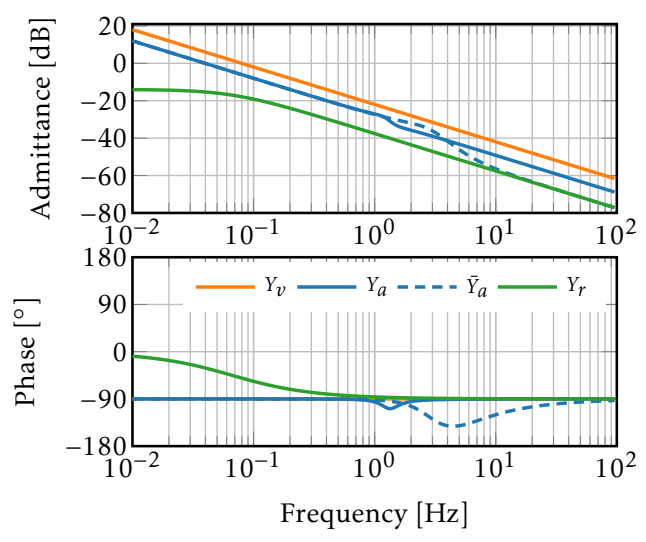

(a)

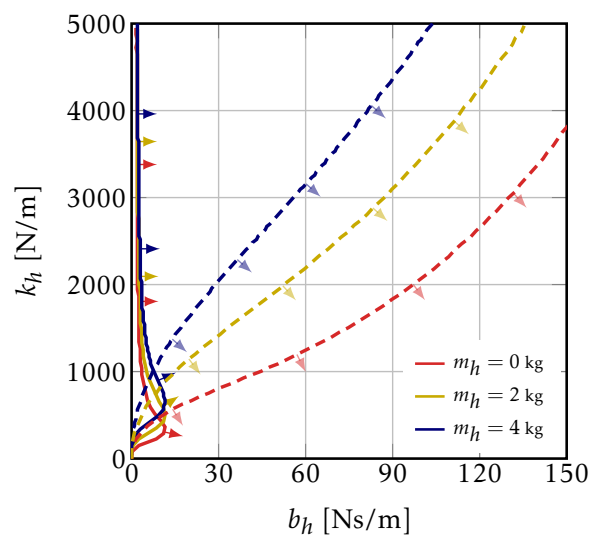

(b)

Figure 4.13: Influence of differential velocity control on system performance and interaction stability. (a) Bode plot to compare a system with band limited differential control $\left(Y_{a}\right)$ with our baseline system $\bar{Y}_{a}$. The parameters are $k_{d}=25$ $\mathrm{Ns}^{2} / \mathrm{m}$ (i.e. $\mathrm{kg}$ ) and $\tau_{d}=0.1 \mathrm{~s}$. Differential control reduces phase lag and improves admittance tracking for high frequencies. (b) Due to the reduced phase lag, the ez-width of $Y_{r}$ (solid lines) is much larger than that of $\bar{Y}_{a}$ (dashed lines). Adding differential control to the velocity controller therefore increases ez-width. The region of stable interaction is indicated by the arrow markings.

Adding a band limited differential velocity controller assists in achieving better high frequency approach of the virtual admittance, as is shown in Fig. 4.13a. Adding differential gain also increases ez-width drastically, as is shown in Fig. 4.13b. This behavior is due to the introduced zero in the transfer function due to the differentiation, and now we can choose the location of the new pole that was introduced by the low pass filter.

\section{Reduce time delays:}

Another method to achieve higher velocity bandwidth in practical setups is to reduce any additional phase lag due to DA conversion $(\mathrm{ZOH})$ or current con- 


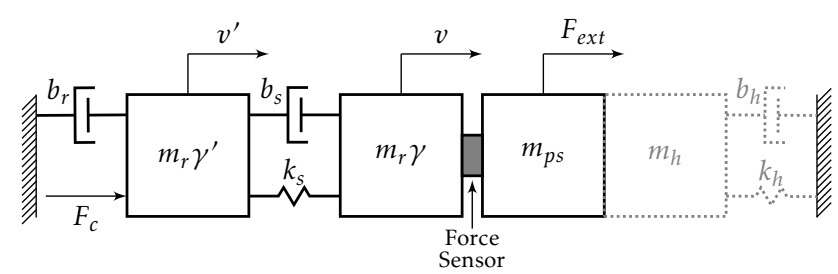

Figure 4.14: Schematic view of a flexible robot, or a system with series elastic actuation. The robot now consists of two inertias $m_{r} \gamma$ and $m_{r}(1-\gamma)=m_{r} \gamma^{\prime}$, connected by structural stiffness $k_{s}$ and damping $b_{s}$ that determine pole location of the lowest resonant mode. The robot can be rigidly connected to a human with inertia $m_{h}$, stiffness $k_{h}$ and damping $b_{h}$, shown by the gray dotted outline

troller delays. The $\mathrm{ZOH}$ dynamics, for a system with sample time $T_{s}$, are given by

$$
H_{\mathrm{ZOH}}=\frac{1-e^{-s T_{s}}}{s T_{s}} .
$$

Which has $-90^{\circ}$ phase lag at the Nyquist frequency $\omega_{N}=\pi / T_{s}$. Increasing the sampling frequency, reducing $T_{s}$, will increase the velocity loop bandwidth. Any pure delay of the form $e^{-s T_{d}}$ has $-90^{\circ}$ phase lag when $\omega T_{d}=\pi / 2$. Decreasing $T_{d}$ will move the excessive phase lag to higher frequencies and increase ez-width. Adding sufficient proportional velocity controller gain counteracts the phase lag introduced by the $\mathrm{ZOH}$ or pure delays, and can makes the system passive for accommodation and stiffness control.

\subsubsection{Guideline 7: Optimize for Robot Stiffness}

If we consider a flexible robot with a low frequency resonant mode (below the controllers Nyquist frequency), we can model this as two inertias sharing a fraction $\gamma$ and $1-\gamma$ of the total robot inertia. The distal $m_{r} \gamma$ and proximal $m_{r}(1-\gamma)=m_{r} \gamma^{\prime}$ are connected by a structural stiffness $\left(k_{s}\right)$ and damper $\left(b_{s}\right)$ (see Fig. 4.14. The force sensor is now non-collocated with the actuator. If $\gamma=0$, this system reduces to an admittance controller for a series elastic actuator, where $k_{s}$ is actually the stiffness of the series elastic element that is used as a force sensor. See Appendix $\mathrm{C}$ for the equation of motion for such a system. 


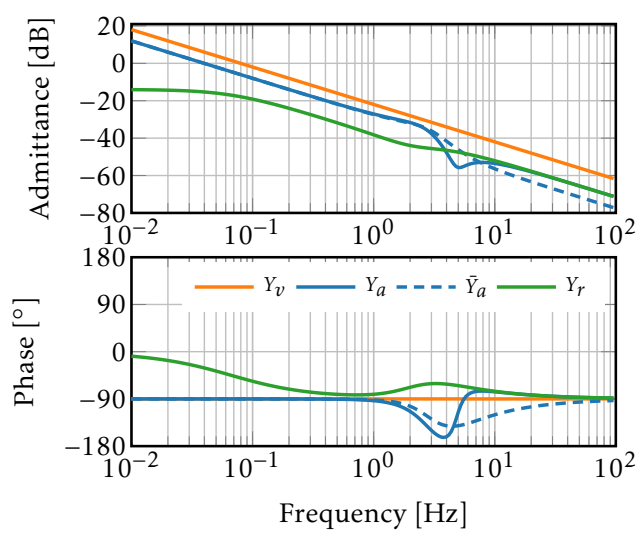

(a)

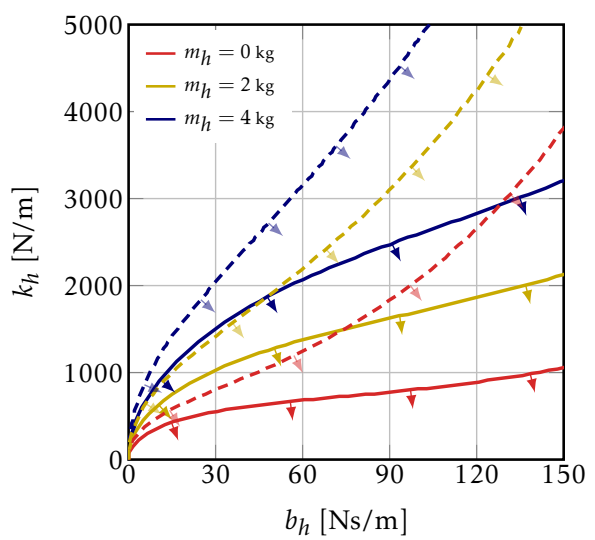

(b)

Figure 4.15: The influence of having a system with finite internal stiffness on system performance and interaction stability (a) This bode plot shows a system with finite stiffness $\left(Y_{a}\right)$ and our baseline system $\bar{Y}_{a}$. The parameters are $\gamma=0.5$, $k_{s}=1000 \mathrm{~N} / \mathrm{m}$ and $b_{s}=100 \mathrm{~N} / \mathrm{m}$. Finite internal stiffness generates more phase lag. (b) Due to the added phase lag, the ez-width of $Y_{r}$ (solid lines) is lower than that of $\bar{Y}_{r}$ (dashed lines). Finite internal stiffness of the robot therefore reduces ez-width. The region of stable interaction is indicated by the arrow markings.

According to Colgate [143] the inertia cannot be passively reduced to any inertia smaller than $m_{r} \gamma$. Except for the condition $\gamma \in[0,1]$, the found passivity conditions are too complex to draw straightforward conclusions.

The performance with a high-frequency mode is acceptable (see Fig. 4.15a). The ez-width is sensitive to $\gamma, b_{s}$ and $k_{s}$. The ez-width gets smaller when lowering internal stiffness, lowering internal damping and increasing $\gamma>0$, as is shown in Fig. 4.15b. This hints at the fact that series elastic actuation, with low $\gamma$, where the force sensor is the spring, should be well doable for admittance controlled system.

\subsubsection{Summarizing Remarks}

The guidelines to achieve minimal apparent inertia of an admittance controlled robot are summarized in Table 4.3, showing the main motivation why it helps to follow them. 
Table 4.3: Summary of the seven guidelines presented in Sec. 4.5.3, together with the main motivation.

\begin{tabular}{|c|c|c|}
\hline $\mathrm{N}^{\mathbf{o}}$ & Guideline & Motivation \\
\hline 1 & use feed-forward control & $\begin{array}{l}\text { effectively lowers the robot inertia to be re- } \\
\text { duced by the admittance controller }\end{array}$ \\
\hline 2 & avoid force filtering & $\begin{array}{l}\text { introduces excessive phase-lag onto } \\
\text { marginally passive virtual inertia model }\end{array}$ \\
\hline 3 & compensate post-sensor inertia & $\begin{array}{l}\text { reduces the apparent inertia but signifi- } \\
\text { cantly reduces coupled stability margins }\end{array}$ \\
\hline 4 & use some virtual damping & $\begin{array}{l}\text { allows for better low-frequency tracking of } \\
\text { admittance }\end{array}$ \\
\hline 5 & modify the velocity reference & $\begin{array}{l}\text { non-physical phase-lead can give better } \\
\text { tracking of pure inertial model and in- } \\
\text { creases coupled stability margins }\end{array}$ \\
\hline 6 & increase velocity loop bandwidth & $\begin{array}{l}\text { pushes the excessive phase lag to higher } \\
\text { frequencies, which requires higher environ- } \\
\text { ment stiffness to destabilize the coupled } \\
\text { system }\end{array}$ \\
\hline 7 & optimize for robot stiffness & $\begin{array}{l}\text { internal resonant modes introduce phase } \\
\text { lag between force sensor measurement and } \\
\text { velocity measurement }\end{array}$ \\
\hline
\end{tabular}

\subsection{DIsCUSSION}

Naive haptic admittance controllers that use only feedback control achieve passivity with good approach of the intended dynamics, when rendering pure virtual stiffness or pure damping. Such controllers have difficulty rendering pure inertia lower than the original device inertia. This is inconvenient, since the admittance control paradigm is commonly used to attempt inertia reduction of bulky devices. The analyses in this chapter, our experience and reports in literature show that attempted inertia reduction leads to coupled instability. With a feedback-only velocity controller, admittance controllers become unstable when the device is firmly held by humans (e.g. for cooperative industrial tasks or haptic displays) or when it is attached to limbs (e.g. for rehabilitation devices). However, completely avoiding feedback control is infeasible, since it is required to suppress unwanted disturbances from external forces and friction forces. 
The guidelines presented in this work propose several solutions to this coupled instability problem when rendering virtual inertia lower than the device inertia. The goal of these guidelines is to simultaneously 1) achieve a better approach of the apparent dynamics to the intended virtual dynamics, and 2) ensure robust coupled stability in the sense of passivity. The guidelines give a qualitative description of how to design key parameters of the mechanical system and control system. These were derived from the fact that the design has to be close to passivity, but also approach the intended dynamics properly with sufficient disturbance rejection. We did not discuss proper controller design (i.e. choices for tuning feedback gains). Any objective in terms of robustness or optimality could be used for determining feed-back controller gains, as long as these are within uncoupled stability bounds, and interaction stability bounds given in this work. The ez-width or passivity bounds should be used as optimization constraints during such controller design.

Using the presented framework for designing admittance controlled systems has several limitations. We derived most of the guidelines from an idealized stiff and single-DOF robot. In multi-DOF robots, energetic coupling between non-linear degrees of freedom could result in instability effects absent in single DOF analyses. A dynamical model with distributed mechanical compliance might be more useful in practical cases. However, the analysis for a system with a single resonant mode leads to qualitatively non-informative and complicated conditions for passivity, uncoupled stability and interaction stability. For a distributed flexible model this would be even more so. Nevertheless, while the conditions might seem complicated, they could be incorporated in design software.

In practice, velocity measurements required for velocity control can be performed by tachometers (EMF based), or gyroscopes. The more common alternative of numerical differentiation of joint position encoder signals with high spatial resolution leads to quantized and noisy estimates of joint velocity. Such a noisy estimate result in a noisy or grindy feel when interacting with the robot. Low-pass filtering this quantization noise results in unwanted resonance in the PI velocity controller's feedback loop and jeopardizes passivity. Therefore, estimation methods that use optimal integration of joint position measurements, joint acceleration estimations and a model of the device could give a joint velocity estimation with low phase lag and a high signal to noise ratio.

However, measuring estimating the robot accelerations, also required for guidelines 3 and 6 , can be difficult in practice. We have added first order low-pass filters to indicate limited sensor bandwidth observed in practice. Accelerometers 
output noisy signals, resulting in a noisy feel of the device during interaction. Other acceleration estimation methods, such as double numerical differentiation of joint-encoder measurements yield heavily quantized and noisy estimations as well. Possible state observer models together with optimal sensory integration could aid in obtaining an optimal estimation of the acceleration. Note that guidelines 1 and 5 do not need acceleration measurements. These use the accelerations from the virtual dynamics, which are derived from the force measurements.

The analyses in this work focused mostly on the influence of isolated parameter changes. Coupled parameter changes, for example by using feed-forward control and a low-pass filter on the force concurrently, were not discussed. Applying two guidelines, or changing two system variables could show unexpected interaction.

We briefly discussed the influence of $\mathrm{ZOH}$ and time-delay effects on passivity properties. Using discrete time sub-models for the feedback controller, virtual dynamics and possible state estimators might give slightly different and more realistic passivity conditions. Nevertheless, since haptic devices usually use fast sampling frequencies above $1000 \mathrm{~Hz}$, we assume that the found guidelines are valid.

The post-sensor effects analyzed were assumed to be purely inertial. In practice we notice that post-sensor backlash and flexibility leads to unwanted limitcycles. Whether this behavior is to be expected from the apparent dynamics in combination with coupled post-sensor dynamics, or exhibit a different form of instability, has to be further analyzed. 
Arvid Q.L. Keemink, Niek W.M. Beckers and Herman van der Kooij

The stability was illusory.

— Liu Cixin, The Three-Body Problem, 2006

Abstract-Attempting to reduce inertia with admittance control can result in an unstable system during human-robot interaction. In this work we show the origin of this active behavior. We present a simple stabilization method that activates a virtual damper only if the virtual energy stored in the virtual model becomes higher than the real energy exchanged between the robot and the human. This damper dissipates excess energy built up in the virtual model dynamics, which ensures stable interaction between humans and admittance controlled robots with inertia reduction. Model simulations on a linear one degree-of-freedom system and a validation of the method on a non-linear two degree-of-freedom haptic setup show the simplicity and applicability of the method.

LIST OF USED SYMBOLS AND ABBREVIATIONS

\begin{tabular}{ll}
\hline Symbol & Explanation \\
\hline$\emptyset$ & the empty set or empty subsystem \\
$\mathbf{0}$ & 0-junction, common effort (bondgraph) \\
$\mathbf{1}$ & 1-junction, common flow (bondgraph) \\
$a$ & passivity polynomial coefficient \\
$\mathbf{A}_{b}(t)$ & time-varying system transition matrix \\
$b(\xi)$ & generalized damping coefficient dependent on system state \\
$\hat{b}$ & SPIS damper value \\
$\mathcal{B}_{v}$ & dissipative subsystem of intended (virtual) dynamics \\
$c r$ & subscript: between controller and robot mechanics
\end{tabular}


C

diss subscript: relating to dissipation

e tracking error

ext subscript: relating to external influence

E exchanged energy between two systems

$E_{0} \quad$ energy initially stored in dynamical system

f force vector

$f_{S} \quad$ discrete time sample frequency

F generalized force

subscript: relating to force

$h$ subscript: relating to the human

$h r \quad$ subscript: between human and robot mechanics

$h v \quad$ subscript: between human and virtual dynamics

$H \quad$ energy stored in a system as potential and kinetic energy

I interface through with (virtual) power flows

$\mathbf{J}(\mathbf{q}) \quad$ manipulator Jacobian

$k$

generalized mechanical stiffness, gain or sample instant

K

controller gain matrix

$m, M \quad$ generalized mechanical inertia

subscript: measured

M inertia tensor

MSe modulated source of effort (bondgraph)

MSf modulated source of flow (bondgraph)

$P \quad$ mechanical power

$\mathbf{q}(t) \quad$ vector of joint angles

$r \quad$ subscript: relating to the robot mechanics

$\mathbb{R}, \mathbb{R}^{+} \quad$ set of real numbers, set of positive real numbers

$\mathcal{S} \quad$ switching surface (decision boundary)

$t, \tau \quad$ time

$T \quad$ controller sample time

$v \quad$ generalized velocity

subscript: relating to the virtual model

$\mathbf{v}(t) \quad$ velocity vector 


\begin{tabular}{ll}
$v c$ & subscript: between virtual dynamics and controller \\
$v o l$ & subscript: relating to voluntarily applied fore \\
$x$ & generalized position \\
$\mathbf{x}(t)$ & linear position vector \\
$\mathcal{X}$ & set of achievable states \\
$Y$ & (virtual) dynamical system with admittance causality \\
$z$ & discrete time domain variable of the $z$-transform \\
$\mathbf{z}(t)$ & vector of expanded system state \\
$\mathbf{z}_{\infty}$ & attractor \\
$Z$ & (virtual) dynamical system with impedance causality \\
$\mathcal{Z}$ & set of achievable expanded states \\
$Z^{+}$ & set of the positive integers (incl. 0 ) \\
$\mathbf{Z O H}$ & zero-order-hold dynamical system \\
$\alpha, \beta, \gamma$ & admittance controller parameterization parameters \\
$\Gamma$ & energy dissipated internally in a dynamical system \\
$\delta$ & amount of spring penetration \\
$\delta e(t)$ & difference between stored energy $H_{v}$ and transmitted energy $E_{h r}(t)$ \\
$\epsilon$ & initial distance between inertial mass and springs in Fig. 5.3 \\
$\eta(t)$ & vector of generalized Coriolis and centrifugal forces \\
$\xi(t)$ & vector of system state \\
$\omega$ & angular frequency \\
$\Omega$ & subspace of $\mathcal{Z}$, split by $\mathcal{S}$ \\
\hline &
\end{tabular}

\begin{tabular}{ll}
\hline Abbrev. & Explanation \\
\hline DOF & degree of freedom \\
PD & proportional-derivative \\
PI & proportional-integral \\
PRC & positive real condition \\
SP & strict passivity \\
SPIS & strict passivity inspired stability \\
UUB & uniform upper bound \\
\hline
\end{tabular}




\subsection{INTRODUCTION}

Safety and dependability are key to successful integration of robots into human environments or to use them as wearable power augmentation devices. Haptic interaction control methods, like admittance control, are used to achieve this goal by making a robotic device behave like a safe mechanical impedance during physical human-robot interaction [68, 87].

During admittance control, the robot movement is controlled to follow a motion reference that is derived from how a virtual dynamical model with admittance causality responds to the applied forces from a human operator. In this way, the robot impedance felt by the operator imitates the impedance of this virtual model. A basic version of the control diagram is shown and explained in Fig. 5.1. Admittance control allows strong and heavy robots to be moved by the human operator with ease. Human power augmentation is hence achieved by masking of the drive train friction, reduction of apparent inertia and cancellation of gravity of the the robot itself and lifted tools and objects [94]. Such a controller is relevant for, for example, having a robot lift unknown heavy loads while the human is supposed to feel a constant low inertia.

Attempting to reduce inertia with admittance control with a feedback controller only, by giving the virtual model an inertia value lower than that of the real system, can result in system instability when the robot is in contact with the operator or a stiff environment. This instability during contact, or coupling, can be explained as energetically non-passive (i.e. active) behavior of the robot $[92,63,94,72,88,144]$ (see also Chapter 4, Sec. 4.4).

In this work we show two different viewpoints on causes of active behavior in admittance control, which have been overlooked in literature. We show that recently developed methods in literature to achieve time-domain passivity for admittance control with either time-delays [145] or inertia reduction [146] fundamentally do not solve admittance control's apparent activity problem during inertia reduction. We present a switching damper controller to dissipate at least the actively generated amount energy and achieve stable interaction of an admittance controlled robot in contact with stiff environments while achieving high inertia reduction factors. The introduced damping does, however, reduce interaction performance. 


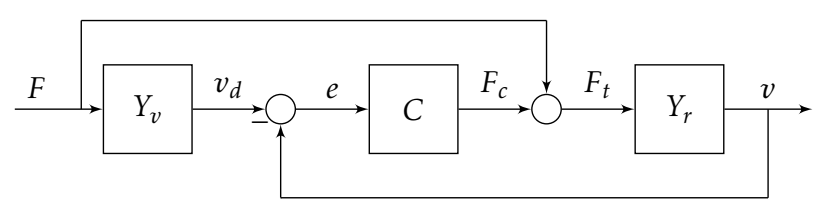

Figure 5.1: Basic admittance controlled system. An external force $F$ is measured and 'applied' on a virtual admittance model $Y_{v}$ which results in desired velocity $v_{d}$. A PI velocity controller $C$ tries to enforce the desired velocity on robot dynamics $Y_{r}$ by applying control force $F_{c}$. The resulting, real velocity is given by $v$. Note that the system would be theoretically equivalent with a PD position controller.

\subsubsection{Passivity and Passivity Enforcement}

Consider a continuous-time mechanical dynamical system with a power-conjugated force and velocity input-output pair $(F(t), v(t))$. The energy exchanged at time $t$ between the coupled operator and this dynamical system is given by

$$
E(t)=\int_{0}^{t} F(\tau) v(\tau) d \tau+E_{0} .
$$

Here $E_{0}$ is the energy initially stored in the system at $t=0$. Positive energy $\left(E(t)>E_{0}\right)$ implies net energy transferred to the system, whereas negative energy $\left(E(t)<E_{0}\right)$ implies net energy transferred to the operator.

For a system to be energetically passive, or just 'passive', the following necessary and sufficient time-domain passivity condition is required

$$
E(t) \geq E_{0} \forall t
$$

This condition implies that the system never returns more energy to the user than the user ever provided to the system.

Passive systems, and interconnections of passive systems, are stable [92]. Involuntary human forces generated by intrinsic limb impedance and reflexes have been found to behave mostly passively [99], although not always [141]. Nevertheless, from everyday experience we know that human interaction with passive objects (e.g. grasping, lifting, pulling or dragging of non-actuated objects in the real world) always leads to stable interaction. Therefore, ensuring passive behavior of a robotic system during physical interaction with a passive 
human operator guarantees interaction stability by putting the responsibility of stable interaction on the human's controller.

Several authors have presented methods to enforce passivity on haptic devices in the frequency domain according to the positive real condition (PRC) $[92,68]$. Time-domain passivity (i.e. upholding (5.2)) was enforced on dynamical simulations or open loop impedance causality type haptic devices by Hannaford and Ryu et al. by damping away excess energy at the man-machine port $[147,148]$. For dynamical simulations with integration methods that would otherwise result in instability, passivity has been enforced by calculating model states back from the Hamiltonian, enforcing energy conservation as a constraint [149].

For admittance control in the form as shown in Fig. 5.1 the PRC dictates that passive inertia reduction is, in fact, impossible. If a high amount of constant damping in the virtual dynamics is used [144] (see Chapter 4), a small amount of passive inertia reduction can be approached for low interaction frequencies. However, adding this required amount (or more) of constant damping does not allow for more passive reduction of the apparent virtual inertia. Furthermore, it results in an over-damped system that is sluggish and is unnecessarily damped when free-floating. A possible solution to this problem is to use computed torque (feed-forward) control [144] (see Chapter 4) in the inner motion control loop. Such control requires proper models of the robotic system, which might be complicated, expensive or intractable for multi degree of freedom (DOF) real systems. Furthermore, the PRC gives usually a black-box condition for passivity, not stating the origin of the active behavior.

Only recently sampled time domain passivity enforcement has been attempted for admittance control $[150,146,145]$. The focus of the work of De Stefano et al. [145] was not on inertia reduction but on compensating the active behavior of time-delays by applying a time-delay-passivity-network. Nabeel et al. [146] built on this idea to attempt using the same method to enforce passivity for inertia reduction systems. The work of Nabeel et al. is highly ambiguous as to which variables at which sample instants are actually required and used; their desired velocity modification results in an algebraic loop that is not properly resolved. Furthermore, the applied method in $[150,146]$ is inherently flawed, as they neglect the actual origin of the active behavior, as will be discussed in this work in detail in Sec. 5.2. Appendix D presents a counter-example to the method presented in [146] that shows that their passivity controller cannot guarantee any kind of passive behavior during inertia reduction due to a wrong assumption about the source(s) of the active behavior. 
In this work we develop a method to yield a stable interaction between an admittance controller that attempts to reduce inertia and an environment with known maximal stiffness. We do this by observing the energy exchanged through the power port at the human-robot interface and the human-virtual model interface (see $\mathcal{I}_{h r}$ and $\mathcal{I}_{h v}$ in Fig. 5.2) and by using a switching virtual damper on the virtual model accordingly. We avoid using extra forces in the low-level velocity/position controller, because these are sometimes non-accessible during design on retrofitted industrial manipulators or when using low-level controllers that perform their own motion control.

The chapter is organized as follows: in Sec. 5.2 we discuss standard admittance control and how different kinds of energy flow through different interfaces. In Sec. 5.3 and Sec. 5.4 we use the relevant energy observed to develop a strict passivity inspired stability (SPIS) controller for admittance control. In Sec. 5.5 we validate the controller stability in simulation and in a real nonlinear, two DOF haptic setup.

\subsection{ENERGETIC BEHAVIOR}

For an admittance controlled robot coupled to a person there are multiple real and virtual energy flows between the human, actuators, virtual model elements and the robot mechanics.

A bondgraph diagram [151] is a concise and insightful way to show energy flows between dynamical elements or subsystems across different physical domains. It can be used to visually and conceptually separate them from signals that only carry information but no energy. Fig. 5.2 shows a bondgraph diagram of the real and virtual power flows and signals used to model an admittance controlled device interacting with a human. The the directed half-arrows $(-$ or $\neg)$ represent power-bonds, where the tip of the half-arrow indicates the defined direction of positive physical power flow between energy sourcing, sinking or storing dynamical elements. Normal arrows $(\rightarrow)$ indicate information carrying signals between elements. In other words, a power bond resembles a bi-directional (i.e. $\leftrightarrows$ ) power-conjugate pair of physical effort (i.e. an across variable, such as mechanical force or electrical voltage) and physical flow (i.e. a through variable, such as mechanical velocity or electrical current) 'signals'. The perpendicular line at the and or start of the half arrow (i.e. $\rightarrow$ or $\mapsto$ ) indicates the direction of the effort. The accompanying flow has to go in the opposite direction. This determines the causality of the connected subsystem: admittance or impedance 


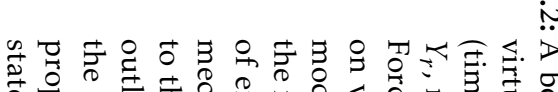
若

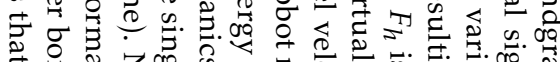

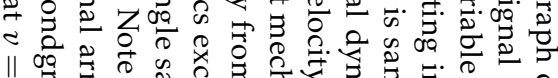

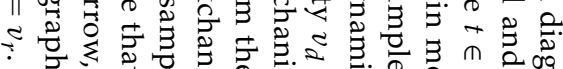

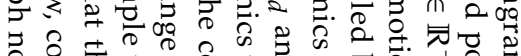

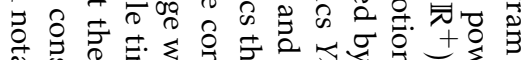

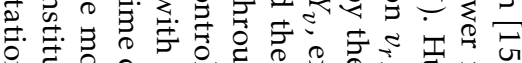

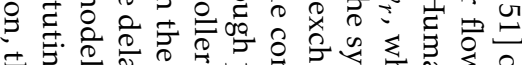

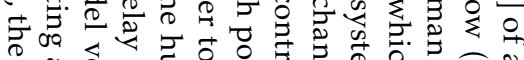

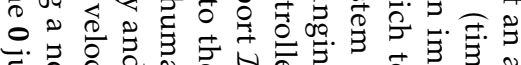

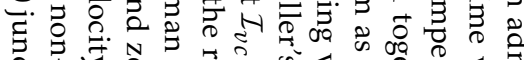

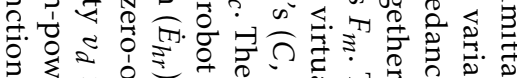

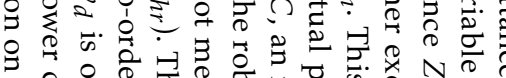

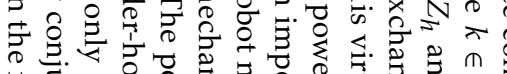

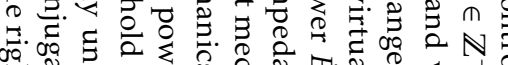

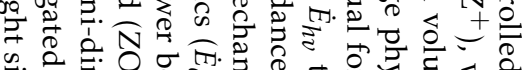

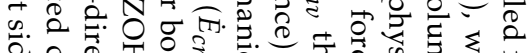

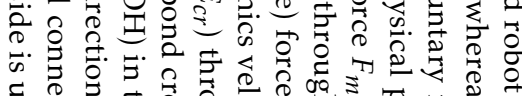

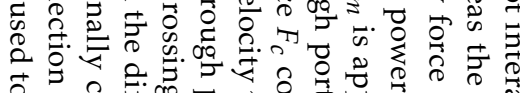
o 0 ठ

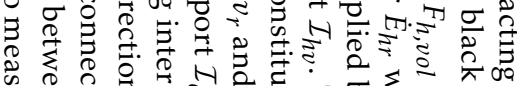

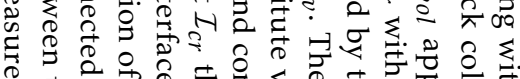

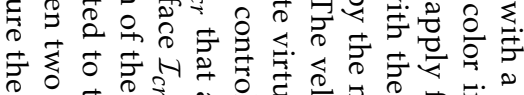

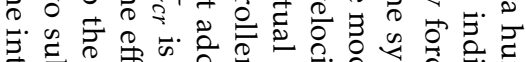

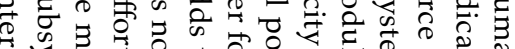

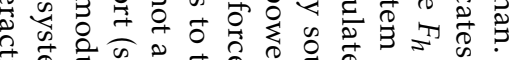

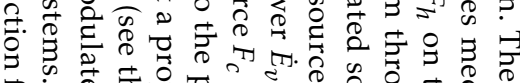

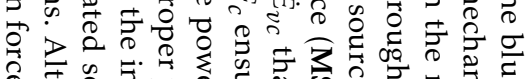

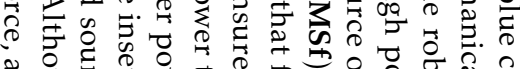

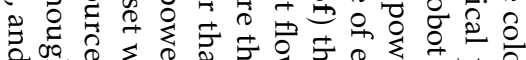

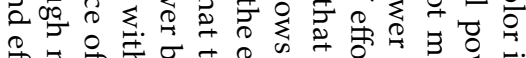

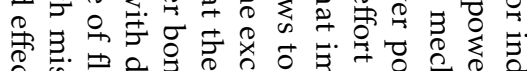
蛋

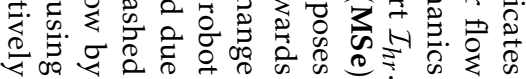

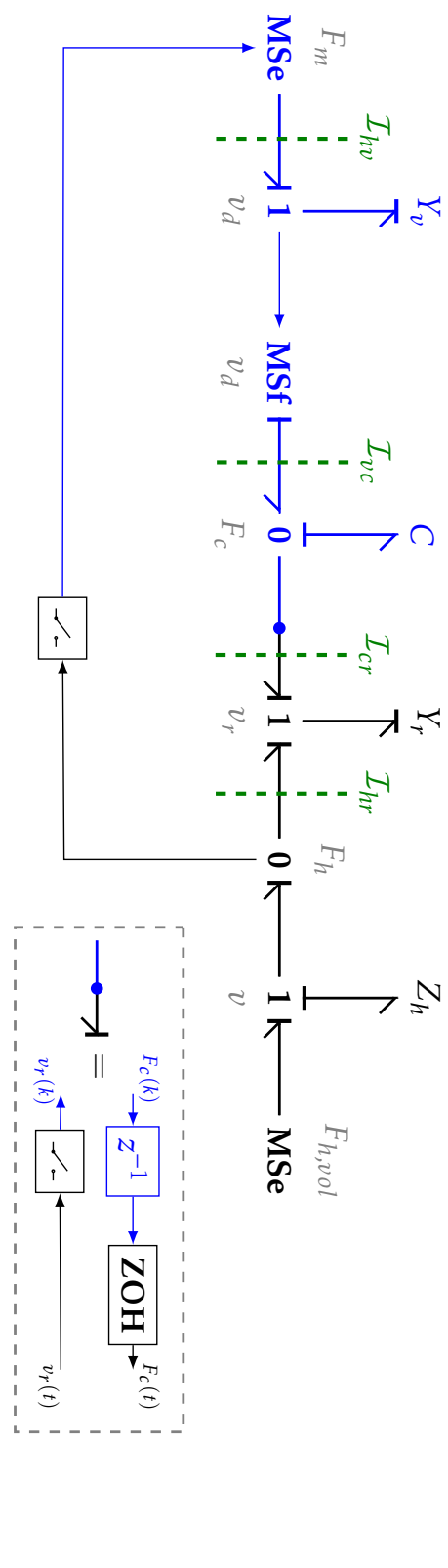


causality. For example, systems with admittance causality have effort-in, flowout causality. Such a system should have the perpendicular line at the end of the half-arrow close to the sub-system with admittance causality (since the force is an input, and the model velocity is the output of an admittance model). The junctions shown by the numbers $\mathbf{0}$ and $\mathbf{1}$ indicate common effort (all connected elements share the same effort, such as serial mechanical springs or parallel electrical impedances) and common flow (all elements share the same flow, such as a spring connected to a mass or electrical impedances in series) respectively. An ideal modulated source of effort (e.g. an ideal variable force/voltage source) is given by MSe, and an ideal modulated source of flow (e.g. an ideal variable velocity/current source) is given by MSf. Passive subsystems with admittance causality are denoted by $Y$, and passive subsystems with impedance causality are denoted by $Z$.

We distinguish four relevant real and virtual power ports that energy passes through, denoted by interfaces $\mathcal{I}$, where interaction between sub-systems takes place. The continuous time index is given by $t$, the discrete time index by $k$. Note that we lump the actuator and controller into a single system and will henceforth talk about energetic behavior of the controller. We also assume that motor torque is controlled with sufficiently high bandwidth such that the controller becomes an ideal effort source [151]. These four power ports are where:

- The controller supplies real energy $E_{c r}(t)$ from the controller to the robot mechanics through $\mathcal{I}_{c r}$.

- The human operator supplies real energy $E_{h r}(t)$ from the human to the robot mechanics through $\mathcal{I}_{h r}$.

- The human operator supplies virtual energy $E_{h v}(k)$ from the human to the virtual model through $\mathcal{I}_{h v}$.

- The virtual model supplies virtual energy $E_{v c}(k)$ from the virtual model to the controller through $\mathcal{I}_{v c}$.

Virtual dynamics $Y_{v}$ are passive, and might contain an intended dissipative subsystem $\mathcal{B}_{v} \subseteq Y_{v}$ that functions as an energy sink. The controller $C$ is commonly a passive PD-position, or PI-velocity, controller. Both the robot's mechanical dynamics $Y_{r}$ and the human's limb impedance $Z_{h}$ are both continuous-time, passive, immitances.

We recognize that the connection between the virtual model and the controller is uni-directional, and therefore not a power-conjugated one (as is also 
mentioned in [152]). This is visible in Fig. 5.2, where the flow source for $v_{d}$ is only uni-directionally connected to the virtual model, without any reaction force acting back on the virtual model.

\subsubsection{The Admittance Controller's Inherently Active Behavior}

The active behavior of a continuous time implementation of an admittance controller* is effectively stated by the restrictions on controller parameters put forth by the PRC. Nevertheless, the PRC's black-box input-to-output passivity analysis does not explain the virtual or physical source of this active behavior for a standard admittance controller. It only states which coefficients need to be greater/smaller than others to achieve passive behavior. Therefore, analysis into the source of the active behavior will prove useful in determining a method to stabilize the coupled system.

\section{A Time-Domain Interpretation:}

Admittance controlled systems that attempt to reduce inertia can become unstable when in contact with stiff environments; e.g. by hitting a hard surface or being tightly held by a human operator. A model of a similar event is shown in Fig. 5.3. An inertial mass $M$ is at rest at position $x(0)=0 \mathrm{~m}$. A constant positive voluntary force from the human $F_{h, v o l}$ is applied to this mass for a duration $t_{F}$ seconds. This accelerates the mass towards the right; to one of the two unilateral compliant elements (i.e. springs resembling e.g. a human hand) at distance $\epsilon_{2}$ from the starting position $x(0)$. This human compliance creates a repulsive spring force $F_{h, s}=-\left(x-\epsilon_{2}\right) k_{h}$ only if $x>\epsilon_{2}$. A second unilateral compliance is added on the opposite side, at a distance of $\epsilon_{1}$ from $x(0)$, to constrain the range of motion. In the limit of $\epsilon_{1}=\epsilon_{2}=0$ the two unilateral compliant elements form a single typical bilateral spring with stiffness $k_{h}$, modeling the robot being held by a human operator.

We define two different cases of this system that will be investigated further:

1. An admittance controlled robot: $M$ is the robot mass $m_{r}$. The admittance controller measures both the human forces $\left(F_{h, v o l}\right.$ and $\left.F_{h, s}\right)$, and applies control force $F_{c}$ to attempt to reduce this inertia to the lower value of $m_{v}$.

${ }^{*}$ In this work we ignore possible extra sources of (virtual) energy that arise due to quantization or sampling. 


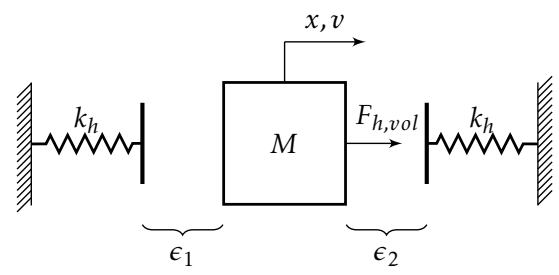

Figure 5.3: Model of a 1D mass $M$ colliding with unilateral human compliance $k_{h}$ after positive (pointing to the right) force $F_{h, v o l}$ acts for a time $t_{F}$. The springs are at initial distance $\epsilon_{1}$ and $\epsilon_{2}$ from the starting position $x(0)=0 \mathrm{~m}$. After $t_{F}$ seconds, the force stops accelerating mass $M$. After moving a distance $\epsilon_{2}$ mass $M$ will collide with the right-most spring. Afterwards, the mass $M$ will bounce towards the other spring and the cycle continues. The position and velocity of the mass are given by $x$ and $v$ respectively. Note that if $\epsilon_{1}=\epsilon_{2}=0$ this is equivalent to a typical mass-spring system with a bilateral spring with stiffness $k_{h}$.

The robot uses a PD position controller to track the reference position $x_{d}(t)$ and velocity $\dot{x}_{d}(t)=v_{d}(t)$ coming from the virtual model:

$$
\begin{aligned}
m_{r} \ddot{x}(t) & =F_{c}(t)+F_{h, s}(t)+F_{h, v o l}(t) \\
m_{v} \ddot{x}_{d}(t) & =F_{h, s}(t)+F_{h, v o l}(t) \\
F_{c}(t) & =k_{p}\left(x_{d}(t)-x(t)\right)+k_{d}\left(v_{d}(t)-v(t)\right)
\end{aligned}
$$

2. The passive intention (denoted as 'intended' or 'intention' in the text): $M$ is virtual mass $m_{v}$, i.e. no admittance control used:

$$
m_{v} \ddot{x}(t)=F_{h, s}(t)+F_{h, v o l}(t)
$$

For both cases, their dynamical behavior is shown in Fig. 5.4. The robot mass was $m_{r}=1 \mathrm{~kg}$, while the virtual mass was $m_{v}=0.5 \mathrm{~kg}$. The controller is a PD position controller with gains $k_{p}=1000 \mathrm{~N} / \mathrm{m}$ and $k_{d}=100 \mathrm{Ns} / \mathrm{m}$. The human compliance was modelled as a linear stiffness $k_{h}=1000 \mathrm{~N} / \mathrm{m}$. The applied force $F_{h, v o l}$ was applied for a duration of $t_{F}=0.1 \mathrm{~s}$. To achieve $1 \mathrm{~J}$ of energy supplied $\left(E_{h r}\right)$ to the passive intended behavior, the force magnitude had to be $10 \mathrm{~N}$. The springs were located at a distance $\epsilon_{1}=\epsilon_{2}=0.2 \mathrm{~m}$ from the mass' initial position.

The intention (case 2) is passive by definition. The mass $m_{v}$ will accelerate, gather momentum and energy due to the applied human force. After moving distance $\epsilon_{2}$ it collides with the spring and penetrates a distance $\delta$ into the spring. 


\section{Position, Velocity and Energy}
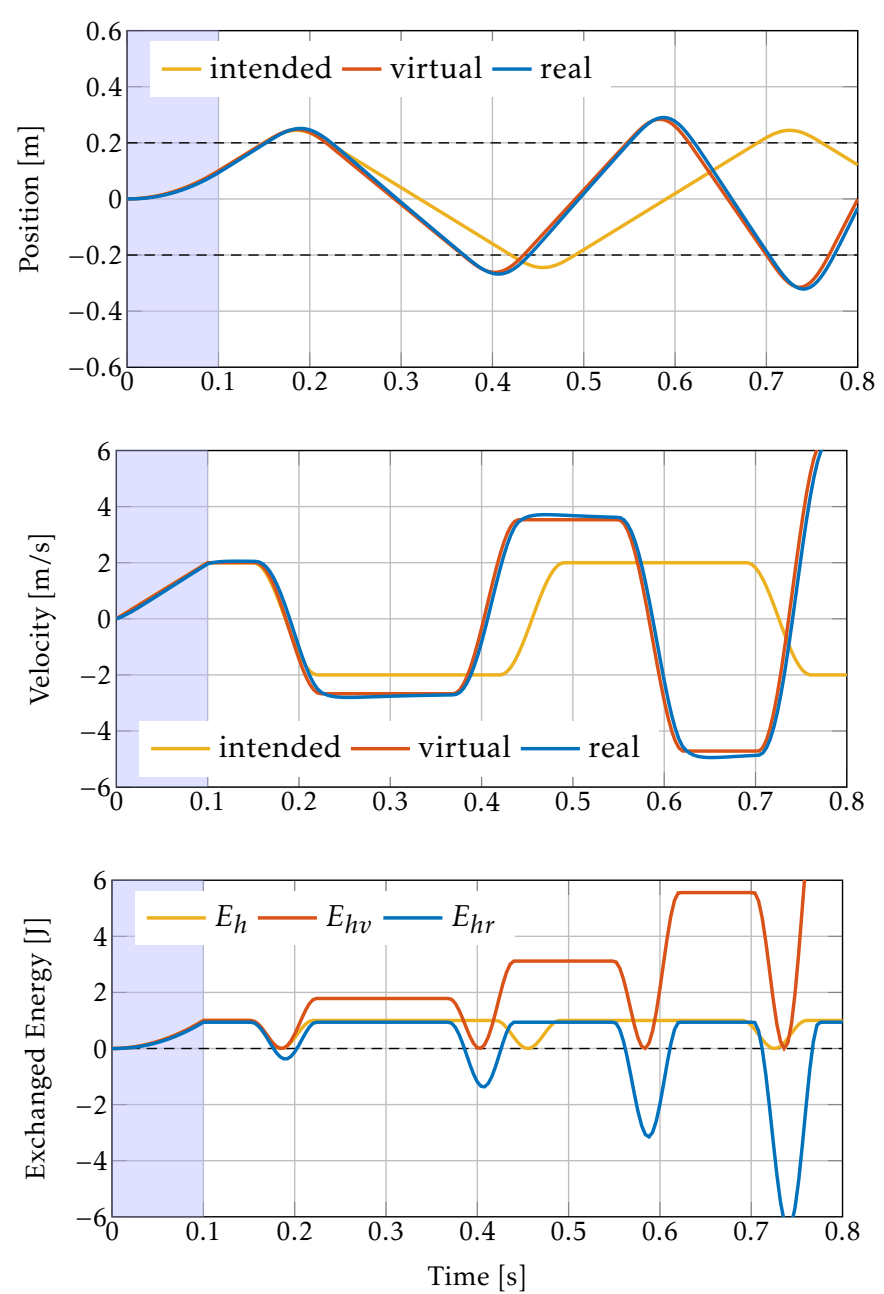

Figure 5.4: Top) The position of the mass. The dashed lines indicate the location where the mass is in contact with the springs. Middle) The velocity of the mass. Bottom) Exchanged energy from human to device through different interfaces $\left(E_{h r}\right.$ and $E_{h v}$, case 1$)$ and what should ideally have been transferred $\left(E_{h}\right.$, the intention, case 2). In the energy plot, the dashed line indicates the threshold for active behavior (see (5.2), with $E_{0}=0 \mathrm{~J}$ ). with positive energy showing passive behavior. The shaded region shows the time when constant force $F_{h}$ was acting on the mass. Note that the intended behavior was passive where the exchanged energy equals the kinetic energy of the mass. The energy exchanged between robot and human $\left(E_{h r}\right)$ becomes negative and shows active behavior. 
The spring will push back the mass towards the spring on the other side, resulting in a marginally stable bouncing cycle. The kinetic energy initially supplied by the human's voluntary force $F_{h, v o l}$ is fully converted to potential energy by the spring $k_{h}$ during collision. This potential energy is in turn converted back to kinetic energy by the spring's repulsive force. There is no net energy gained or lost by the interaction of these passive elements.

During admittance control with inertia reduction (case 1) the mass will accelerate and start to move as intended when the force $F_{h, v o l}$ is applied. The mass will collide with the spring, but penetrates a distance $\delta^{\prime}>\delta$ into the spring. The spring will push the mass towards the spring on the other side. The admittance controlled mass exits the spring with a velocity higher than its incoming velocity, i.e. some kinetic energy is 'created'. Consequently, it hits the other spring where this process repeats. This results in an unstable, or growing, cycle.

The cause of this active behavior is twofold, but both causes are explained by the accumulated phase lag of the inner motion loop in the admittance controller. The motion controller's output force always lags behind the motion reference coming from the virtual model. As a consequence, the human (i.e. the spring $k_{h}$ ) experiences the controlled robot to have more inertia than the $m_{v}$ it was trying to simulate. Therefore:

1. it will penetrate deeper into the spring, experiencing a larger repulsive force $F_{h, s}$ than was intended,

2. the 'robot mass' also spends more time inside the spring than was intended.

Consequently, the reaction force $F_{h, s}$ from the spring acts for a longer amount of time on the virtual model than was intended. This leads to accumulation of more virtual momentum, and therefore more virtual energy, than was intended. This results in a higher velocity when leaving the spring than the initial velocity at the moment of colliding with the spring.

Fig. 5.4 shows that the energy transferred between the robot and user $\left(E_{h r}\right)$ becomes negative during a collision. This implies a net energy transfer to the human operator that is bigger than what the human operator introduced. Every consecutive bounce this amount of energy returned to the human operator increases in magnitude. The human (a pure spring) resupplies all that same amount of energy back to the robot, leading to a never exceeded maximum of approximately $1 \mathrm{~J}$ of kinetic energy physically stored in the robot, like in the intended case. 


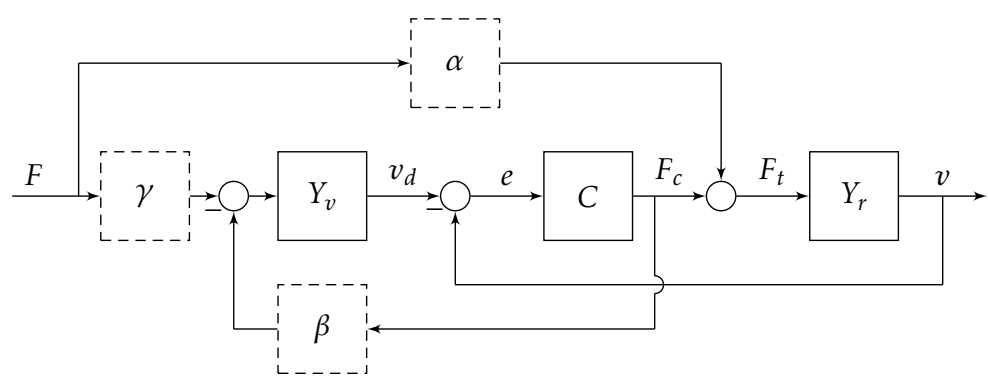

Figure 5.5: Admittance controlled system as shown in Fig. 5.1, but parameterized with binary $\alpha, \beta$ and $\gamma$ that have a value of either 0 or 1 . Parameter $\alpha$ represents backdrivability and should be 1 for a physical system. Parameter $\beta$ represents the bi-directionality of the spring-damper controller in $C$ (i.e. a PD-position of PI-velocity controller), i.e. if $\beta=1$ the controller will also apply reaction forces on the virtual model. Parameter $\gamma$ allows for the copying of the interaction force to the virtual model. A typical admittance controller has $\alpha=\gamma=1$ and $\beta=0$.

The virtual energy transferred between the virtual model and user $\left(E_{h v}\right)$ goes to $0 \mathrm{~J}$ during every collision, like in the intended case. However, after a collision the transferred energy has increased; the excess energy resupplied to the human operator is fed back to the virtual model with a non-trivial amount of scaling.

The energy exchanged between user and robot $\left(E_{h r}\right)$ has the correct (i.e. intended) upper limit, whereas the energy exchanged between the user and virtual model $\left(E_{h v}\right)$ has the correct lower limit. Therefore, an inertia reduced admittance controlled robot that bounces against a stiff surface, or is held in place by finite stiffness, will exhibit active behavior that presents itself as a growing oscillation.

\section{A Systems Interpretation:}

Fig. 5.2 shows that there is a uni-directional connection between the virtual model and the subsequent controller-robot dynamics. This effectively means that the controller impedance $C$, as a PD position controller, acts like a unidirectional spring-damper between the virtual model and the robot dynamics. The robot dynamics feel the virtual model but not vice versa. Consequently we parameterize the controller from Fig. 5.1 with several binary gains $\alpha, \beta$ and $\gamma$. Parameter $\alpha$ turns on/off backdrivability. Parameter $\beta$ adds a 'physically consistent' feedback of the controller (as is shown in Fig. 5.5) to make the controller 
a bilateral spring-damper. Parameter $\gamma$ determines whether the force on the robot is copied onto the virtual model. With these parameters, the PRC gives the following condition for frequency-domain passivity (see Appendix D for the derivation):

$$
\begin{aligned}
a_{2} \omega^{4} & +a_{1} \omega^{2}+a_{0} \geq 0, \forall \omega \\
a_{2} & =m_{v}\left(\alpha m_{v}\left(b_{r}+k_{d}\right)-\gamma k_{d} m_{r}\right) \\
a_{1} & =b_{r}\left(k_{d}^{2} \beta(\alpha \beta+\gamma)-k_{p} m_{v}(2 \alpha \beta+\gamma)\right) \\
a_{0} & =\beta b_{r} k_{p}^{2}(\alpha \beta+\gamma) .
\end{aligned}
$$

For (marginal) passivity, a negative $a_{1}$ can be balanced by both a positive $a_{2}$ and $a_{0}$ for several parameter values. Although we are here not interested in parameter values to determine the passivity boundary, this passivity condition readily tells us three important things:

1. If $\alpha=0$ and $\gamma=1$ then the system is never passive, since this makes $a_{2}<0$, which makes it active for high frequencies.

2. If $\beta=1$ it is 'more passive' than if $\beta=0$, since it makes $a_{0}>0$. So the uni-directional spring $(\beta=0)$ is a source of activity.

3. If $\gamma=0$, with $\alpha=\beta=1$, the system would be always passive; it would be a spring-damper interconnection between two passive systems.

Therefore, it is shown that it is the copying of the force from the human to the virtual model (i.e. the modulated source of effort that acts as an energy source), together with the uni-directional spring-damper from the controller that cause active behavior. This shows why the method of Nabeel et al. [146] cannot work, since it only attempts to make the uni-directional spring-damper element passive, neglecting the active behavior of the modulated effort source. In the work of De Stefano et al. [145] the problem is not circumvented, but solely hidden, by not attempting inertia reduction and performing compensation only for energy introduced due to time-delay. It also shows that solutions developed for teleoperation methods to do negate active effects of time-delay, such as developed in [153], will not work on admittance control, since in those scenarios the force is not copied from the slave side directly onto the master. 


\subsection{STABILITY AND PASSIVITY CONDITIONS FOR ADMITTANCE CONTROL}

In this section we will revisit the passivity condition given in (5.2), and put forth a more strict passivity condition to derive a stabilizing controller that is useful for admittance controlled systems.

We make the notational distinction between energy exchanged through power ports ( $\mathcal{I}$ in Fig. 5.2) denoted as ' $E$ ' and energy stored in systems denoted as ' $H$ '. For example, the energy stored in the virtual system has to be the difference between the energy exchanged between the human and the virtual model $\left(E_{h v}\right)$ and the energy dissipated internally $\Gamma(t)$

$$
H_{v}(t)=E_{h v}(t)-\Gamma(t)
$$

We assume in this and the following derivations that the initial energy $E_{0}=0 \mathrm{~J}$ and that no intended damping behavior is required (i.e. $\mathcal{B}_{v}=\emptyset$ ).

\subsubsection{Strict Passivity (SP)}

Because $H_{v}(t)$ is the virtual energy stored in the system as kinetic energy in the virtual model, this energy has a definite lower bound because it cannot be negative:

$$
H_{v}(t) \geq 0, \forall t
$$

If we would make $H_{v}(t)$ a lower bound for $E_{h r}(t)$, it would follow that $E_{h r}(t) \geq 0$, $\forall t$, which makes the system strictly passive at interaction port $\mathcal{I}_{h r}$. Therefore, a sufficient condition for strict passivity (SP) is

$$
E_{h r}(t) \geq H_{v}(t) \geq 0, \forall t
$$

In this way, the typical (marginal) passivity condition in (5.2) is upheld at the human robot interface $\mathcal{I}_{h r}$. Relating to the results in Fig. 5.4, trying to enforce SP amounts to forcing the 'real' $\left(E_{h r}\right)$ and 'virtual' $\left(E_{h v}\right)$ energy to at least coincide. Note that in Fig. 5.4 the virtual energy was the exchanged virtual energy $E_{h v}(t)$ instead of stored virtual energy $H_{v}(t)$. However, in a damping free system these are equivalent. 
The SP condition in (5.4) is useful, but more strict than the typical passivity condition in (5.2). Because, even if $H_{v}(t) \geq 0$, and $H_{v}(t) \geq E_{h r}(t) \geq 0$, the SP condition would not hold but system is still passive according to (5.2).

\subsubsection{Strict Passivity Inspired Stability (SPIS)}

SP requires that the energy difference $\delta e(t)=H_{v}(t)-E_{h r}(t)$ should go to, or below, $0 \mathrm{~J}$. This can be attempted in the following way:

- Decreasing $H_{v}(t)$ : by damping away energy in the virtual model $H_{v}(t)$ will decrease its value towards, or below, $E_{h r}(t)$. Moreover, the human might interact with the virtual damper behavior through $\mathcal{I}_{h r}$, resulting in forced energy dissipation, in turn increasing $E_{h r}(t)$ as preferred. Because the sates in $Y_{v}$ are a function of $H_{v}(t)$, this will cause a state change, such as a lower desired velocity. This might result in reducing $\dot{E}_{h r}(t)$, resulting in slower reduction of $\delta e(t) \rightarrow 0$. Especially for low virtual model velocities this becomes less effective.

The aforementioned agrees qualitatively with the analysis described in Sec. 5.2.1; the mass motion should be damped in either the virtual model, or in the real system $^{\dagger}$, to spend less time inside the spring to reduce the accumulation of excess momentum and kinetic energy.

To force $\delta e(t) \rightarrow 0$ we put a damper (attached to ground) on the virtual model $Y_{v}$ only when $H_{v}(t) \geq E_{h r}(t)$. Because the motion-control-loop has finite bandwidth and possible tracking overshoot, it cannot be guaranteed that the velocity of the robot $v(t)$ goes to 0 as quickly as the virtual model velocity $v_{d}(t)$. For high enough damping values this will force $\delta e(t) \rightarrow 0$ if $H_{v}(t) \geq 0$, but cannot enforce $E_{h r}(t) \geq H_{v}(t)$ at all times. Therefore the switching damping controller enforces Strict Passivity Inspired Stability, or SPIS.

We will show in the next section that this method cannot be used to make the system passive. It can, however, make it stable in the sense that it exhibits bounded oscillatory behavior.

\footnotetext{
${ }^{\dagger}$ Forcing an increase in $E_{h r}(t)$ through the low-level controller, instead of the virtual model, might also help in making the system strictly passive. However, this will not be discussed in this work.
} 


\subsection{LINEAR DYNAMICAL ANALYSIS OF SPIS}

In this section we will analyse the dynamics of a linearized, ideal robot that is admittance controlled, with SPIS enforced. The robot will be attached to a linear spring with stiffness $k_{h}$. We assume that the energy exchanged through interfaces $\mathcal{I}_{h r}$ and $\mathcal{I}_{h v}$ can be properly determined, or determined with known uncertainty, in a real system by measuring robot interaction force and motion states. See Appendix D for how this energy bookkeeping is carried out.

\subsubsection{System Dynamics}

The system dynamics are assumed to be autonomous and linear, with state $\xi(t) \in \mathcal{X} \subseteq \mathbb{R}^{4}$, given by state vector and state equation

$$
\begin{aligned}
& \xi(t)=\left[\begin{array}{llll}
x(t) & v(t) & x_{d}(t) & v_{d}(t)
\end{array}\right]^{T} \\
& \dot{\xi}(t)=f(\xi)=\mathbf{A}_{b}(\xi) \xi(t) .
\end{aligned}
$$

The system matrix $\mathbf{A}_{b}(\boldsymbol{\xi})$ is derived from (5.3), being

$$
\mathbf{A}_{b}(\xi)=\left[\begin{array}{cccc}
0 & 1 & 0 & 0 \\
-\frac{k_{p}}{m_{r}}-\frac{k_{h}}{m_{r}} & -\frac{k_{d}}{m_{r}} & \frac{k_{p}}{m_{r}} & \frac{k_{d}}{m_{r}} \\
0 & 0 & 0 & 1 \\
-\frac{k_{h}}{m_{v}} & 0 & 0 & -\frac{b(\xi)}{m_{v}}
\end{array}\right],
$$

where

$$
b(\xi)=\left\{\begin{array}{l}
\hat{b}, \text { if } E_{h r}(t)<H_{v}(\xi) \\
0, \text { if } E_{h r}(t) \geq H_{v}(\xi),
\end{array}\right.
$$

with $\hat{b}>0$. Eq. (5.5) denotes the switching damper 'controller' used to enforce SPIS. 


\section{Augmented System Dynamics}

The state vector is augmented with human-robot interaction energy $E_{h r}(t)$. The new state vector is denoted by $\mathbf{z}(t) \in \mathcal{Z} \subseteq \mathbb{R}^{5}$, and it is given by

$$
\mathbf{z}(t)=\left[\begin{array}{c}
\xi(t) \\
E_{h r}(t)
\end{array}\right], \dot{\mathbf{z}}(t)=\left[\begin{array}{c}
\dot{\xi}(t) \\
\dot{E}_{h r}(t)
\end{array}\right]=\left[\begin{array}{c}
\mathbf{A}_{b}(\xi) \xi(t) \\
-k_{h} x(t) v(t)
\end{array}\right] .
$$

This augmented system is autonomous with (5.5) describing a switching surface $\mathcal{S} \subset \mathcal{Z}$, where

$$
\mathcal{S}=\left\{\mathbf{z}(t) \mid E_{h r}(t)=H_{v}(\mathbf{z})\right\},
$$

splits $\mathcal{Z}$ into two regions $\Omega_{0}$ (where $b(\xi)=0$ ) and $\Omega_{\hat{b}}$ (where $b(\xi)=\hat{b}, \hat{b}>0$ ). On the switching surface the damper value switches between 0 and $\hat{b}$ or vice versa, depending on $\dot{\mathbf{z}}(t)$.

If SP could be enforced, all trajectories would never be able to leave $\Omega_{0}$. We would then demand that all trajectories only enter $\Omega_{0}$ through $\mathcal{S}$ and never exit, i.e. we would enforce that

$$
-\nabla\left(H_{v}(\mathbf{z})-E_{h r}(t)\right) \cdot \dot{\xi}(t)>0 .
$$

I.e., the inner product of the inward normal of the switching surface $\mathcal{S}$ and the system rate $\dot{\mathbf{z}}(t)$ would need to be positive. This gives the condition

$$
b(\xi) v_{d}^{2}(t)+k_{h} x(t) v(t)\left(v_{d}(t)-v(t)\right) \geq 0, \forall t,
$$

which effectively equals

$$
P_{\text {diss }}(t)-F_{h}(t)\left(v_{d}(t)-v(t)\right) \geq 0, \forall t .
$$

This shows again that from a time-domain perspective (see Sec. 5.2) it is the velocity error, combined with the spring force, generating energy that leads to non-strictly-passive behavior. This energy should henceforth be dissipated by a virtual damper $b(\xi)$ that dissipates with power equal to $P_{\text {diss. }}$. However, we cannot guarantee that $P_{\text {diss }}(t)>F_{h}(t)\left(v_{d}(t)-v(t)\right)$ at all times, due to possibly small values of $v_{d}(t)$ that require unreasonably high values of $b(\xi)$. It also shows that an uniform upper bound (UUB) on the velocity error would limit the active 
behavior of the system, explaining the apparent success of Morbi et al. [154] to limit the active behavior of an admittance controlled system.

\section{Stability of SPIS}

Eq. (5.5) acts like a sliding-mode-like controller to make the system converge to a stable cycle during interactions with the pure spring environment. This controller will make the interaction stable and cannot make it generally passive.

The initial conditions of the admittance controlled system are always such that $x(0)=x_{d}(0), v(0)=v_{d}(0)$ and $E_{0}=E_{h r}(0)=g(v(0), x(0))$ with $g(\cdot)$ some function of the initial states. Therefore the system always starts with zero tracking error and starts by definition on switching surface $\mathcal{S}$.

If $\mathbf{z}(t) \in \Omega_{\hat{b}}$, and $\hat{b}$ was chosen in such a way that $\mathbf{A}_{b}(\boldsymbol{\xi})$ is asymptotically stable, then by linear Lyapunov argument that system will converge to the point $\mathbf{z}_{\infty}=\left[\begin{array}{lllll}0 & 0 & 0 & 0 & E_{0}\end{array}\right]^{T} \in \Omega_{0}$. Therefore, all trajectories $\mathbf{z}(t) \in \Omega_{\hat{b}}$ are initially attracted to move towards $\Omega_{0}$, and will hit $\mathcal{S}$ in finite time.

If $\mathbf{z}(t) \in \Omega_{0}$, or on $\mathcal{S}$, The dynamics $\mathbf{A}_{b}(\xi)$ with $b(\xi)=0$ is unstable. Therefore, $\mathbf{z}(t)$ will then not converge to $\mathbf{z}_{\infty}$ and will slide on $\mathcal{S}$ or be ejected out of $\Omega_{0}$ in finite time, through $\mathcal{S}$, back into $\Omega_{\hat{b}}$. From this moment on, the cycle repeats.

For dynamics $\mathbf{A}_{b}(\xi)$ that are stable when $\mathbf{z}(t) \in \Omega_{\hat{b}}$, but that have 'too little' damping, the instability when in $\mathbf{z}(t) \in \Omega_{0}$ adds more energy to the system than is dissipated when $\mathbf{z}(t) \in \Omega_{\hat{b}}$ before hitting $\mathcal{S}$ again. The cycle will grow and eventually the trajectories will not enter $\Omega_{0}$ anymore because $E_{h r}$ becomes more negative over time. Trajectory $\mathbf{z}(t)$ will then stay in stable $\Omega_{\hat{b}}$ and the cycle will stop growing. The exchanged energy from the robot to the user will, nevertheless, have become more negative. This cycling behavior for 'too little' damping is an outward converging cycle.

For 'sufficiently high' values of $\hat{b}$ the energy generated by the instability when $\mathbf{z}(t) \in \Omega_{0}$ can be dissipated when $\mathbf{z}(t) \in \Omega_{\hat{b}}$ before hitting $\mathcal{S}$. In this way, cycling behavior ensures that the trajectories pass through both $\Omega_{0}$ and $\Omega_{\hat{b}}$ indefinitely. This leads to an inward converging cycle.

The switching damper $b(\xi)$ acts as a sliding-mode-like controller, such that the apparent dynamics are mostly that of $\mathcal{S}$. Therefore, this switching system will show stable cycling behavior, as is intended for the discussed dynamics shown in Fig. 5.3 and 5.4. The only necessary condition for a converging cycle is that $\hat{b}$ is chosen such that $\mathbf{A}_{b}(\boldsymbol{\xi})$ is at least marginally stable when $\mathbf{z}(t) \in \Omega_{\hat{b}}$. There is no straightforward method to calculate the required minimal value of 
$\hat{b}$ to ensure an inward converging cycle. Therefore, its value needs to be found numerically, or it can be set to any sufficiently high value.

\section{Response to Active Environments}

The analysis in Sec. 5.4.1 holds for an autonomous system, e.g. an admittance controlled system interacting with a passive pure spring. However, to be useful the method should also work when in contact with an active human limb. Any voluntary forces of the human can introduce energy into, or extract energy from, the virtual model and robot dynamics through both interfaces $\mathcal{I}_{h v}$ and $\mathcal{I}_{h r}$. This is shown by the force $F_{h, v o l}$ from the modulated source of effort (MSe) in Fig. 5.2.

Although the energetic behavior of an active human force is unpredictable, we reason that using the switching damper from (5.5) results in stable interaction in that case as well. If the human supplies energy $\left(\dot{E}_{h r}(t)>0, \dot{E}_{h v}(t)>0\right)$ both $E_{h r}(t)$ and $E_{h v}(t)$ will increase. If, due to unstable behavior, $E_{h v}(t)$ increases above $E_{h r}(t)$, the switched damper starts dissipating energy $(\Gamma(t))$ until $H_{v}(t)=E_{h v}(t)-\Gamma(t)$ is smaller than $E_{h r}(t)$, if possible. If $E_{h r}(t)$ becomes negative (active behavior) the stored energy $H_{v}(t)$ cannot become negative, but only approach a value of $0 \mathrm{~J}$; i.e. a stationary system with $v_{d}=0 \mathrm{~m} / \mathrm{s}$. This gives the system time to dissipate enough energy to achieve $\dot{E}_{h r}(t)>0$ before allowing new motion. Hence, the interaction between the human limb and the unstable system is hereby stabilized.

\subsection{VALIDATION}

In this section we validate the switching damper in simulation and in a two DOF haptic setup. The simulated dynamics are those described in the analysis. The real system, however, is not autonomous in the sense that a human operator moves the robot and can introduce energy into the system or extract it.

\subsubsection{Simulation}

The numerical simulation of the admittance controller was performed in Matlab 2015 b with a variable-step continuous-time dynamics solver. The model parameters were $m_{r}=1 \mathrm{~kg}, m_{v}=0.1 \mathrm{~kg}, k_{d}=100 \mathrm{Ns} / \mathrm{m}, k_{p}=1000 \mathrm{~N} / \mathrm{m}$. The admittance controller hence attempts an inertia reduction of a factor of 10. A damper value $\hat{b}=5 \mathrm{Ns} / \mathrm{m}$ makes $\mathbf{A}_{b}(\boldsymbol{\xi})$ strictly stable when in $\Omega_{\hat{b}}$, for an inward con- 


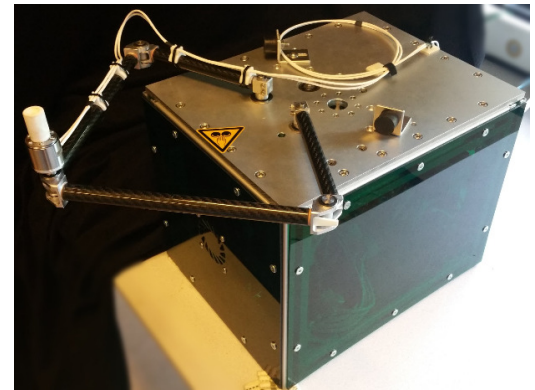

(a)
Workspace, Max. Apparent Physical Inertia [kg]

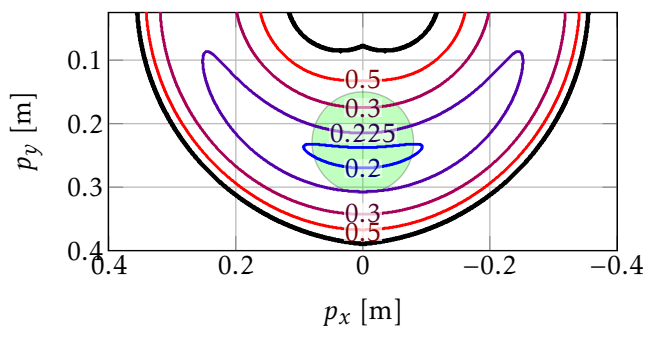

(b)

Figure 5.6: a) The used haptic setup. b) Workspace of the used haptic setup with the workspace boundary shown in black. During the experiment, the end-effector was forced to remain inside the shaded green circle. The level sets of estimated maximum apparent physical inertia (the inertia felt by the user) are shown for values of $0.2,0.225,0.3$ and $0.5 \mathrm{~kg}$. The maximum and minimum inertia were calculated as the maximum and minimum eigenvalue of the manipulator mass matrix expressed in Cartesian coordinates. The lowest maximum physical inertia value across the workspace is $0.185 \mathrm{~kg}$, located near coordinate $p=[0,0.25]^{T}$. The minimal physical inertia (not shown) in the used circular workspace was between $0.16-0.2 \mathrm{~kg}$. The maximal physical inertia was around $0.4 \mathrm{~kg}$. The virtual inertia was set to be $0.05 \mathrm{~kg}$. Therefore, inertia reduction factors were therefore estimated to lie between 3.2 and 8 .

verging cycle of the switching system. A damping value of $2.8881 \mathrm{Ns} / \mathrm{m}$ makes $\mathbf{A}_{b}(\boldsymbol{\xi})$ marginally stable when in $\Omega_{\hat{b}}$, for an outward converging cycle of the switching system. The model started with initial position of $x=x_{d}=0 \mathrm{~m}$ velocity $v=v_{d}=1 \mathrm{~m} / \mathrm{s}$ and initial energy $E_{h r}(0)=H_{v}(0)=0.05 \mathrm{~J}$.

\subsubsection{Haptic Setup}

The SPIS method was also validated on a two DOF haptic setup (see Fig. 5.6a). The setup consists of two actuated joints, which are denoted by the vector $\mathbf{q}(t) \in$ $\mathbb{R}^{2}$ (in rad). They drive a parallel kinematic chain, known as a five-bar linkage. The links connected to the motor have a kinematic length of $153 \mathrm{~mm}$. The subsequent links connected to the end-effector have a kinematic length of 238 $\mathrm{mm}$. Each joint is driven by a Faulhaber 3890-048-CR, spaced $70 \mathrm{~mm}$ apart. Each motor is driven by high-bandwidth current control by a Technosoftmotion 
iPOS4808 BX-CAT driver over an EtherCAT bus. The motors are connected to the links through a 1:7.3 capstan transmission. Absolute encoders at the joints measure joint position with a resolution of $2^{16}$ values per revolution. Forces on the end-effector were measured by a ME-Systeme 6-axis K6D27 100N/2Nm force/torque sensor, read by a GSV-1A8USB K6D amplifier connected to the EtherCAT bus. The control software was implemented in Simulink 2016b, and compiled for TwinCAT3 real-time target software that interfaces with the hardware. The control software ran at a sample frequency of $f_{s}=1000 \mathrm{~Hz}$.

The continuous-time (time index $t$ ) dynamics and discrete-time (discrete-time index $k$, sample duration $T=1 / f_{s}=1 \mathrm{~ms}$ ) jointspace PD position control of the setup are given by:

$$
\begin{aligned}
\mathbf{M}_{q}(\mathbf{q}) \ddot{\mathbf{q}}(t)+\eta(t) & =\boldsymbol{\tau}(k T)+\mathbf{J}^{T}(\mathbf{q}) \mathbf{f}_{\mathrm{ext}}(t) \\
\boldsymbol{\tau}(k T) & =\mathbf{K}_{p}\left(\mathbf{q}_{d}(k T)-\mathbf{q}(k T)\right)+\mathbf{K}_{d}\left(\dot{\mathbf{q}}_{d}(k T)-\dot{\mathbf{q}}(k T)\right),
\end{aligned}
$$

with $\mathbf{M}_{q}(\mathbf{q}) \in \mathbb{R}^{2 \times 2}$ the jointspace mass matrix, $\ddot{\mathbf{q}}(t)$ and $\dot{\mathbf{q}}(t)$ the angular accelerations and velocity respectively, $\eta(t) \in \mathbb{R}^{2}$ the vector of Coriolis and centrifugal forces, $\tau \in \mathbb{R}^{2}$ the actuator torques, $\mathbf{J}(\mathbf{q}) \in \mathbb{R}^{2 \times 2}$ the manipulator Jacobian, $\mathbf{f}_{\mathrm{ext}} \in$ $\mathbb{R}^{2}$ the external force applied by the user, and $\mathbf{K}_{p}=\operatorname{diag}[10,10] \mathrm{Nm} / \mathrm{rad}, \mathbf{K}_{d}=$ $\operatorname{diag}[0.1,0.1] \mathrm{Nms} / \mathrm{rad}$ diagonal proportional and derivative feedback gains respectively.

Jointspace reference $\mathbf{q}_{d}(t), \dot{\mathbf{q}}_{d}(t)$ is determined through an inverse kinematic mapping of an operational space reference $\left(\mathbf{x}_{d}(\mathrm{t})\right.$ and its time derivatives) to jointspace $\left(\mathbf{q}_{d}(t)\right.$ and its time derivatives). The operational space motion reference comes from the discrete-time Cartesian two DOF virtual model, expressed by semi-implicit Euler integration ${ }^{\ddagger}$ :

$$
\begin{aligned}
& \mathbf{v}_{d}(k+1)=\mathbf{v}_{d}(k)+T \mathbf{M}_{v}^{-1}\left[-b(k) \mathbf{v}_{d}(k)+\mathbf{f}_{\mathrm{ext}}(k)\right] \\
& \mathbf{x}_{d}(k+1)=\mathbf{x}_{d}(k)+T \mathbf{v}_{d}(k+1) .
\end{aligned}
$$

We assume a scalar $b$, isometric over the used workspace. The virtual mass matrix is given by isometric $\mathbf{M}_{v}=\operatorname{diag}\left(\left[m_{v}, m_{v}\right]\right)$, where $m_{v}=0.05 \mathrm{~kg}$. This gives between a 3.2 to 8 times reduction in apparent inertia of the setup (see Fig. 5.6b).

\footnotetext{
‡Compared to the explicit (forward) Euler method, the semi-implicit Euler method is a first order symplectic integration method and almost conserves the energy in a dynamical system, making it an almost passive numerical integration scheme. Leap-frog integration (i.e. velocity Verlet) is an alternative symplectic integrator of second order and a has even better energy conserving properties. Nevertheless, active behavior due to the energy generated in by numerical integration is negligible in this work.
} 
The virtual stored energy of the end-effector is calculated by

$$
H_{v}(k)=\frac{1}{2} \mathbf{v}_{d}^{T}(k) \mathbf{M}_{v} \mathbf{v}_{d}(k)
$$

The damper $b(k)$ is switched from a value of $0 \mathrm{Ns} / \mathrm{m}$ to $\hat{b}=20 \mathrm{Ns} / \mathrm{m}$ when $H_{v}(k) \geq E_{h r}(k)$. This amount of damping was found empirically to be sufficient for stable interaction with an admittance controller that reduces the end-point inertia to $0.05 \mathrm{~kg}$. Theoretically, it would be stable for linear stiffness up to approximately $175 \mathrm{~N} / \mathrm{m}$.

\subsection{RESULTS}

In this section we discuss the results from simulation and experiment.

\subsubsection{Simulation Results}

The results in Fig. 5.7 show the behavior described in Sec. 5.4.1. For $\hat{b}=5 \mathrm{Ns} / \mathrm{m}$, the system is able to dissipate enough energy when in $\Omega_{\hat{b}}$ to have the cycle converge inward. The cycle settles, while continuously switching between $\Omega_{\hat{b}}$ and $\Omega_{0}$, showing oscillatory behavior of both the virtual model and the robot dynamics. This oscillatory behavior is preferred, since the virtual dynamics and the environment together are a mass-spring system without damping, for which undamped oscillatory behavior is expected. For $\hat{b}=2.8881 \mathrm{Ns} / \mathrm{m}$, the system is not able to dissipate enough energy when in $\Omega_{\hat{b}}$ to have the cycle converge inward. An increasing amount of energy is transferred to the user $\left(E_{h r}(t)\right.$ becoming more negative), until trajectories $\mathbf{z}(t)$ do not enter $\Omega_{0}$ anymore. However, since $\Omega_{0}$ is by construction marginally stable, the system will converge to stable cycling behavior.

\subsubsection{Haptic Setup Measurement Results}

The results in Fig. 5.8 compare a typical admittance controller $(\hat{b}=0 \mathrm{Ns} / \mathrm{m}$ always) and the admittance controller with the switching damper from (5.5). Fig. 5.8a-c show how for typical admittance control the system became unstable quickly after touching the end effector by hand with a piece of soft foam. The system was aggressively unstable and could be touched only briefly and not 


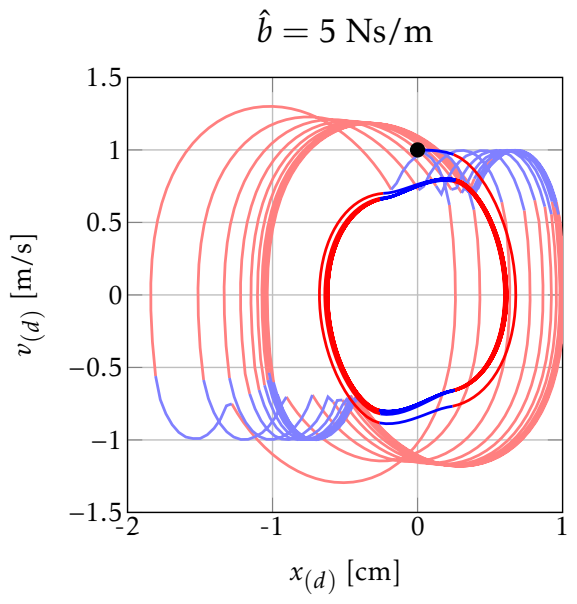

(a)

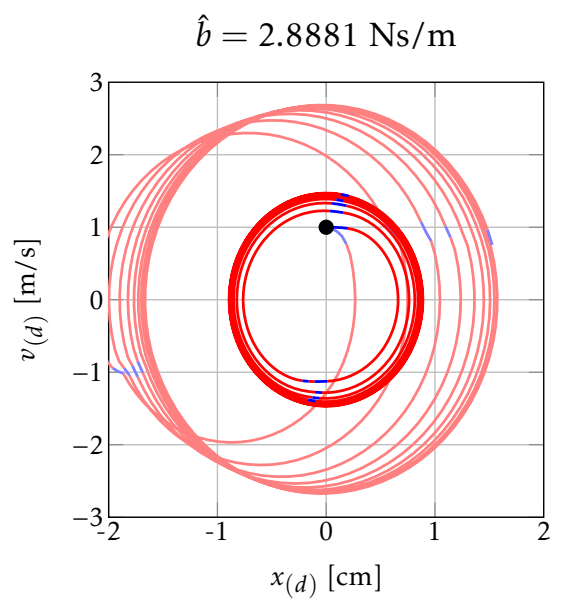

(c)

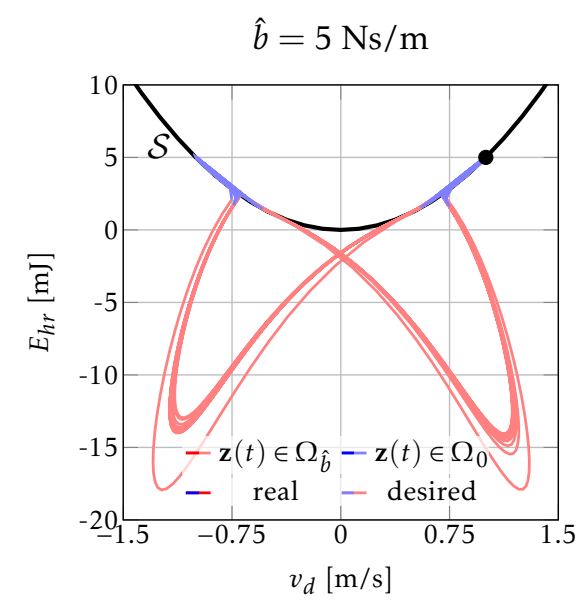

(b)

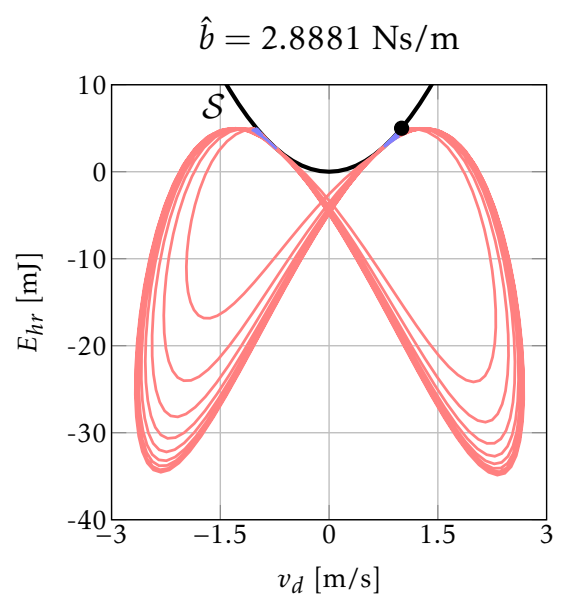

(d)

Figure 5.7: Projections of solutions $\mathbf{z}(t)$ onto $\mathbb{R}^{2}$. Red hues for solutions in $\Omega_{\hat{b}}$ and blue hues for solutions in $\Omega_{0}$. a) and c) Phase diagram, showing stable limit cycles for robot position-velocity $(x-v)$ as normal red/blue hues, and in the desired robot position-velocity $\left(x_{d}-v_{d}\right)$ in light red/blue hues. b) and d) showing the desired velocity versus the exhanged energy between robot and human $\left(v_{d}(k)-E_{h r}(k)\right)$, with switching surface $\mathcal{S}$ shown in black. In b) the trajectories hit $\mathcal{S}$, while in d) they do so only at the beginning, but after some time they become tangent to $\mathcal{S}$. This is outward cycling behavior in c) and d) that forces the system to ultimately stay in $\Omega_{\hat{b}}$ forever, being at least marginally stable. The value of the trajectories at time $t=0$ is given by the black marker in all subfigures. The legend in $b$ ) holds for all subfigures. 


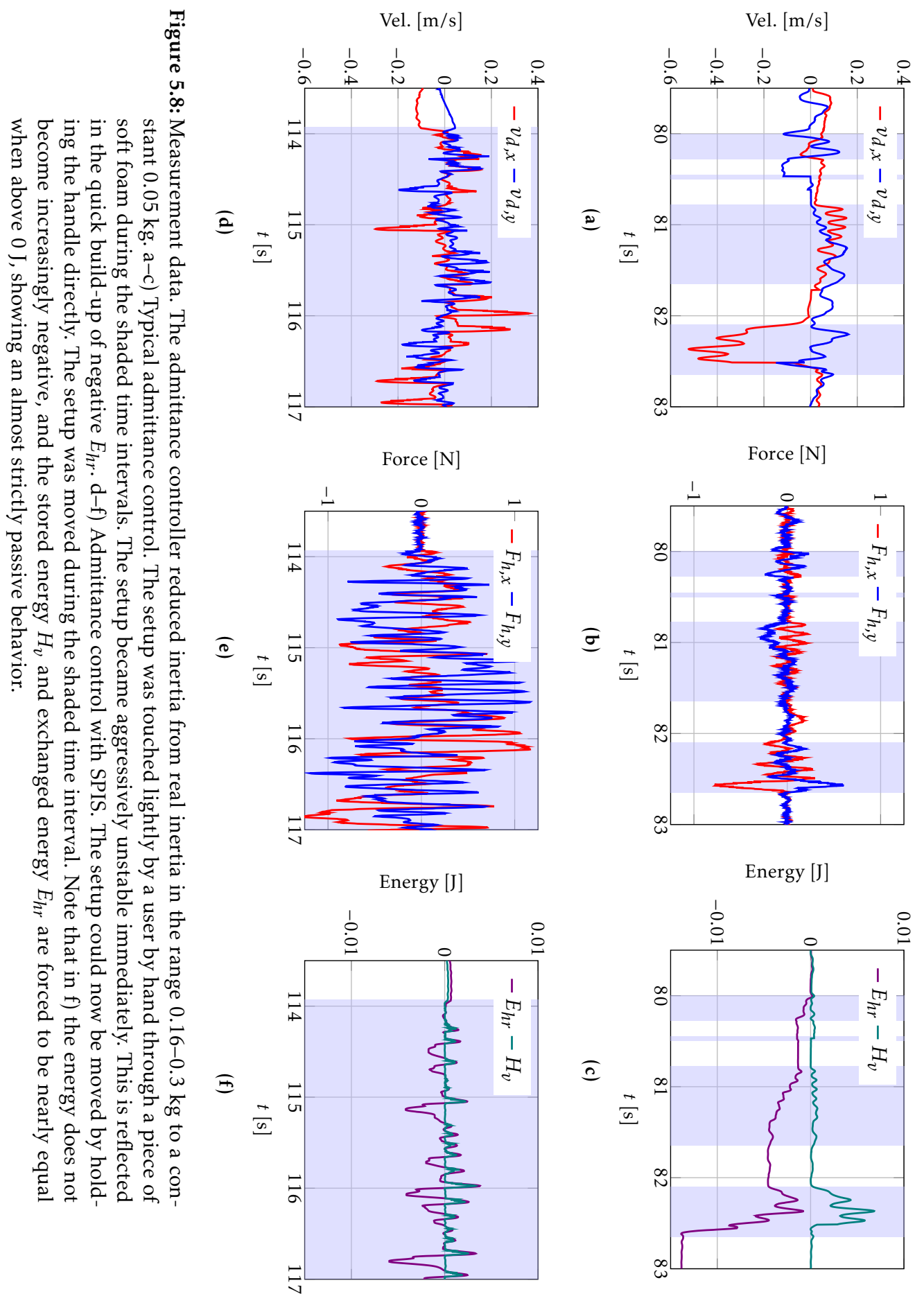


by a human hand directly. While touching the manipulator end-effector, some energy is introduced into the system to make it move. However, more energy is afterwards returned to the user, as is shown by $E_{h r}$ in Fig. 5.8c. The energy exchanged between the setup and the human becomes more negative over time, showing the active behavior introduced by the instability.

Fig. 5.8d-f show measurements performed on the same setup, but with SPIS $(\hat{b}=20 \mathrm{Ns} / \mathrm{m})$. In this case, the setup could be properly held by the operator without it becoming aggressively unstable. Force measurements for this experiment show higher force peak magnitude in Fig. 5.8e, compared to Fig. 5.8b. This happened because the setup could be properly held and moved by a user. Also, the switching damper introduces some mechanical impedance that induces forces on the force sensor due to the human's impedance. The stored energy $H_{v}$ and exchanged energy $E_{h r}$ in Fig. 5.8f are similar in value when both are positive. Also, the real exchanged energy $\left(E_{h r}\right)$ does not become more negative over time (as happens in Fig. 5.8c), showing stable and almost passive behavior.

\subsection{DISCUSSION}

The proposed SPIS admittance controller was shown to be able to stabilize interaction of an admittance controlled system with inertia reduction being in contact with a stiff environments in simulation, and during real physical humanmachine interaction.

\subsubsection{Limitations and Practicalities}

It was not yet shown that this method works in general for virtual dynamics that have compliant elements and intended internal dissipation. It is expected, however, that the method is as applicable in those cases as well, since the amount of virtual potential energy is known. Furthermore, admittance control can simulate compliance passively [144] (see Chapter 4, Sec. 4.5.4).

The amount of required damping $\hat{b}$ can be set a priori to achieve a cycle that converges neither inward nor outward. Analytical stability proofs that complement the qualitative reasoning in Sec. 5.4.1 might shed some light on this issue and give conditions on the minimally required value of $\hat{b}$. However, in practice any high value that ensures a stable system is sufficient (as is shown in the results in Fig. 5.7 and Fig. 5.8), although increasingly higher values of damping 
result in more aggressive dissipation and higher impedance forces on the setup due to stuttering.

This leads to another apparent problem of the implementation of the current method that uses solely feedback control. The choice to use a low virtual inertia comes from the demand for low interaction impedance. Active behavior generated by this inertia reduction (i.e. impedance reduction) requires added impedance from the switching damper to stay stable. As a consequence there still significant device impedance. Possibly feed-forward contributions to the controller could reduce the required impedance to stay stable (see Chapter 4 , Sec. 4.8).

Three practical approaches to determining the proper amount for $\hat{b}$ are as follows: 1) adding a constant amount of damping to a typical admittance controller until the system shows no interaction instability across all practical operator stiffness values. The system will then be impeding and dissipative due to this damper. The SPIS method will then effectively switch off this damper when it is not required to dissipate energy for stabilization. 2) Adaptively increasing $\hat{b}$ when the exchanged energy between human and robot is negative when device velocity is zero. 3) If the force sensor at the interaction port is made compliant, its stiffness becomes the upper bound for the all environment stiffness values the device will ever interact with. This allows to calculate and set a fixed value for $\hat{b}$ when the device dynamics are known. This has as a drawback, however, that the point of interaction with the device becomes compliant, something that might not be preferred.

\subsubsection{Comparison with Literature}

In this work it was shown that the controller proposed by Nabeel et al. [146] cannot guarantee passivity at the human-robot interface when inertia reduction is attempted (see Appendix D for a counter-example to their method). Their method, similar to the one used by De Stefano et al. [145] is only sufficient to handle energy generated due to time-delays, but cannot remove the exact amount of energy to make an admittance controller with inertia reduction passive. The main reason why their method cannot be applied is that the system is not solely active due to the unilateral connection between virtual model and the controller, but also due to the copying of the measured force that acts on the robot mechanics onto the virtual dynamics. Any proper controller that makes 
the system passive should therefore act on both the virtual model as on the real robot mechanics.

\subsubsection{Comparison with Impedance Control}

Time domain passivity enforcement of open-loop impedance controlled haptic devices that have to display virtual environments has been reported in literature. If a bondgraph (similar to 5.2) would be drawn of an impedance controlled system, it would be apparent that the system connections are physically consistent (albeit that possible inertia, damping and stiffness reduction through open loop impedance control requires negative element values). Because impedance controlled devices respond with a reaction force to motion, its active behavior is in their case solely due to time discretization of the virtual model dynamics. Therefore passive sampling methods [149] and adding damping forces at the motor side [147] are sufficient to counteract active behavior and make the system passive.

For admittance controlled systems, however, we have noticed the interconnection between subsystems is non-physical on two levels: copying of the interaction force and the uni-directionality of the controller. On top of that, minor energy-sources due to sampling, delays and integration methods also introduce more energy into the system in both the virtual model and by the controller onto the robot mechanics.

\subsubsection{Future Work}

The current SPIS method makes the system neither passive, nor strictly passive, because damping takes place in the virtual model alone. Adding extra damping in the low-level position control might assist in increasing $E_{h r}$ in (5.2) and (5.4), possibly making the robot passive or strictly passive. However, this requires access to low level motion controllers, something that might not available. Furthermore, it is not trivial to determine how to divide the dissipation amount between the two dampers while being in contact with an unknown environment. Therefore we will investigate this in future work. 


\subsection{CONCLUSION}

In this work we presented a method to enforce stability of an admittance controlled system that attempts to reduce inertia when in contact with stiff environments. The method relies on keeping track of the exchanged energy of the human with the robot and the energy stored in the virtual dynamics. If the energy stored in the virtual dynamics is higher than the exchanged energy, a constant damper dissipates energy from the virtual model. This allows for stable interaction behavior through a simple method that does not require modifications to a low level motion controller. 

Part III

SHARED CONTROL:

DAMPING FORCES AROUND

REACHING TARGETS 

USING POSITION DEPENDENT DAMPING FORCES

AROUND REACHING TARGETS FOR TRANSPORTING

HEAVY OBJECTS: A FITTS' LAW APPROACH

Arvid Q.L. Keemink, Rik I.K. Fierkens, Joan Lobo-Prat, Jack S.F. Schorsch, David A. Abbink, Jeroen B.J. Smeets and Arno H.A. Stienen

It is easier to resist at the beginning than at the end.

- Leonardo da Vinci

Abstract-Passive assistive devices that compensate gravity can reduce human effort during transportation of heavy objects. The additional reduction of inertial forces, which are still present during deceleration when using gravity compensation, could further increase movement performance in terms of accuracy and duration. This study investigated whether position dependent damping forces (PDD) around targets could assist during planar reaching movements. The PDD damping coefficient value increased linearly from 0 $\mathrm{Ns} / \mathrm{m}$ to $200 \mathrm{Ns} / \mathrm{m}$ over $18 \mathrm{~cm}$ (long PDD) or $9 \mathrm{~cm}$ (short PDD). Movement performance of reaching with both PDDs was compared to damping free baseline conditions and to constant damping $(40 \mathrm{Ns} / \mathrm{m})$. Using a Fitts'-like experimental design 18 subjects performed a series of reaching movements with index of difficulty: $3.5,4.5$ and 5.5 bits, and distances 18, 23 and 28 cm for all conditions. Results show that PDD reduced (compared to baseline and constant damping) movement times by more than $30 \%$ and reduced the number of target reentries, i.e. increasing reaching accuracy, by a factor of 4 . Results were inconclusive about whether the long or short PDD conditions achieved better task performance. However, mean human acceleration forces were higher for the short PDD, hinting at marginally faster movements. Overall, PDD is a useful haptic force to get humans to decrease their reaching movement times while increasing their targeting accuracy.

This chapter was published as: A.Q.L. Keemink, R.I.K. Fierkens, J. Lobo-Prat, J.S.F. Schorsch, D.A. Abbink, J.B.J. Smeets and A.H.A. Stienen, "Using position dependent damping forces around reaching targets for transporting heavy objects: A Fitts' law approach", IEEE/RAS-EMBS International Conference on Biomedical Robotics and Biomechatronics (BioRob), 2016. The first two authors contributed equally to this work. 


\begin{tabular}{ll}
\hline Symbol & Explanation \\
\hline$a$ & Fitts' law intercept \\
$b$ & Fitts' law reciprocal data transmission rate \\
$D$ & reaching distance \\
ID & index of difficulty \\
$M T$ & movement time \\
$p$ & probability \\
$r_{m}$ & Fitts' law regression coefficient, taking all values per subject \\
$r_{m}$ & $\begin{array}{l}\text { Fitts' law regression coefficient, taking only mean metric value per } \\
\text { subject }\end{array}$ \\
target width or diameter \\
$\alpha$
\end{tabular}




\subsection{INTRODUCTION}

Understanding physical interaction between humans and mechatronic devices can be useful in the field of teleoperation, rehabilitation and power augmentation robotics. Shaping the interface dynamics and reflected forces when interacting with (virtual) objects (i.e. haptics) allows for task performance optimization. For example, gravity and inertia compensation can reduce physical effort during lifting tasks, and haptic shared control can increase situational awareness and task accuracy in remote transportation tasks [155]. Although interactive robotic manipulators are extremely versatile in their applications and functionality, such as the admittance controlled devices used in industry [156], they pose an inherent safety risk during physical interaction with users. Passive systems, comprised of mechanically passive elements such as springs and dampers/brakes, could be preferred over active systems for specific tasks. Advantages of passive systems include the reduced design complexity, increased inherent safety (although energy can be stored in springs), reduced (maintenance) costs, and increased mobility due to reduced weight and power demands. Examples of passive systems are the mobile Lockheed Fortis [157] in industry, the stationary rehabilitation exoskeleton Dampace [28], and the ankle-foot exoskeleton by Collins et al. [158].

Compared to active systems, the types of forces that can be generated with purely passive systems are limited. Such limitation is partially overcome in Cobot systems by scaling and changing passive dynamical properties accordingly through continuous variable transformers $[104,66]$.

We propose a system inspired by the Cobot systems, but for reaching-like tasks. Controlled damping forces around a reaching target (position dependent damping, or PDD) will be used to optimize a person's motion during humanmachine cooperative tasks of picking and placing of heavy objects. The term 'controlled' implies the use of a physical passive element of which its dissipative properties are varied, e.g. by controlled disk brakes or varying a moment arm of a dash-pot damper. The term 'optimize' implies that movements become faster and easier to perform with higher accuracy and subjective likability. A potential industrial application of PDD would be assisting cooperative transportation of heavy objects with a passive gravity compensation mechanism to a target location by a human operator, where the target is determined by range finding sensors. The PDD could then be used for faster and more accurate placement of heavy objects by the human operator. 
In this study we investigated how PDD changes the human's reaching strategy and performance in terms of reaching time, accuracy and applied force during Fitts-like $[159,160]$ reaching tasks. Additionally, we evaluated whether Fitts' law still holds during reaching with PDD. We used the PDD to help them brake the reaching movement and improve reaching accuracy and therefore movement performance.

\subsection{BACKGROUND}

Modeling human reaching is challenging due to nonlinear arm and muscle dynamics, high muscle redundancy and the required optimal integration of both visual and proprioceptive information [161,162]. Fitts proposed a simple linear relationship that models speed-accuracy trade-offs in rapid, aimed two-way tapping [159] or one-way reaching [160] movements:

$$
M T=a+b \cdot I D .
$$

This relationship describes the movement time $(M T)$ as a function of Index of Difficulty $(I D)$ and two empirical coefficients $a$ and $b$, which are dependent on environment properties. In [163] it is proposed to use the Shannon formulation of the $I D$ (in bits) as a function of reaching distance $D$ and target size/diameter $W$ around that target:

$$
I D=\log _{2}\left(\frac{D}{W}+1\right)
$$

which we will use in the remainder of this work.

Few studies investigated validity of Fitts' law in dissipative environments. In $[164,165]$ it was shown that moving underwater (a dissipative, but highly inertial environment) can be described by Fitts' law with minor modification. Further studies $[166,167,168]$ investigated the influence of constant friction and damping on the movement times. They found that for small masses (below $2 \mathrm{~kg}$ ) a friction force could reduce reaching times and increase accuracy in a Fitts type targeting tasks.

In contrast to the aforementioned studies, we are interested in moving heavy masses (12.5 kg, motivated by the industrial application) and in position dependent damping forces around the reaching target, instead of constant damping or friction forces. 


\begin{tabular}{|c|c|c|c|c|c|c|c|c|}
\hline 18 & 63 & 63 & 18 & 63 & 18 & 63 & 18 & 63 \\
\hline $\mathrm{F}$ & B1 & C\# & W1 & C\# & W2 & C\# & W3 & B2 \\
\hline
\end{tabular}

387

Figure 6.1: Trial blocks presented to the subject. Numbers above the blocks indicate number of trial repetitions. F: Familiarization; B1: Baseline1 condition (nondamping); C1, C2 and C3: Damping condition 1-3 with either constant damping or specific PDD; W1, W2 and W3: Washout phase 1-3 without damping; B2: Baseline 2 condition (nondamping). Dashed outlines indicate these blocks are not used for data analysis.

\subsection{METHODS}

\subsubsection{Experimental Conditions}

In this experiment we compared three damping conditions ( $\mathrm{C} 1, \mathrm{C} 2$ and $\mathrm{C} 3$ ) where subjects performed reaching tasks with different damping forces. These conditions were also compared to two baseline conditions (B1 and B2) without any damping forces during the reaching task. These baselines were used to observe possible overall performance improvements during the complete experiment.

During reaching tasks in conditions C1, C2 and C3, subjects would feel different viscous damping forces $F_{d}($ in $\mathrm{N})$ :

$$
F_{d}=-b(x) v
$$

where $b(x)$ (in Ns/m) is a damping coefficient (d.c.), possibly dependent on position $x$ (in $\mathrm{m}$ ) for PDD, $v$ (in $\mathrm{m} / \mathrm{s}$ ) is the instantaneous velocity of the moved manipulandum. The minus sign in (6.3) shows that this force opposes the motion and makes the dynamical system passive. The d.c. was dependent on conditions as shown in Fig. 6.2. Baselines B1 and B2 had zero d.c. Condition C1 had constant d.c. of $40 \mathrm{Ns} / \mathrm{m}$. Conditions $\mathrm{C} 2$ and C3 had $0 \mathrm{Ns} / \mathrm{m}$ d.c. at the start of the movement, ramping up linearly to $200 \mathrm{Ns} / \mathrm{m}$ over a distance of $18 \mathrm{~cm}$ (C2) or $9 \mathrm{~cm}$ (C3) from the target at $D$. The d.c. was never lower than $0 \mathrm{Ns} / \mathrm{m}$, nor higher than 200 Ns/m. Familiarization (F) and washouts (W1, W2 and W3) had zero d.c. 


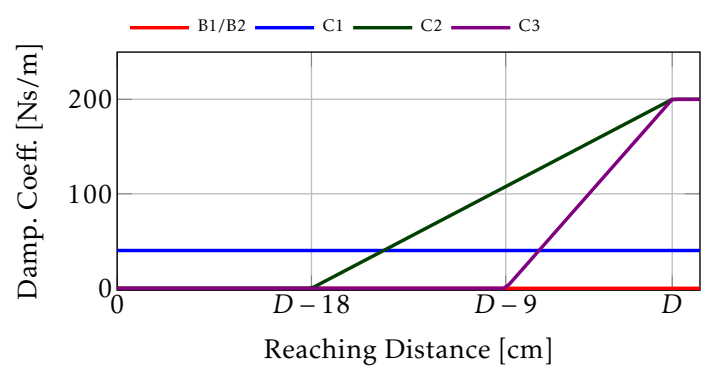

Figure 6.2: Damping coefficients for each condition. Conditions B1 and B2 have zero damping coefficient. Condition $\mathrm{C} 1$ has a constant coefficient of $40 \mathrm{Ns} / \mathrm{m}$. Conditions C2 and C3 increase linearly towards the target point from $0 \mathrm{Ns} / \mathrm{m}$ to $200 \mathrm{Ns} / \mathrm{m}$.

\subsubsection{Experimental Protocol}

After a detailed explanation of the purpose and procedure of the experiment, subjects performed a total of 378 reaching movements (i.e. trials) with a haptic manipulandum (see Sec. 6.3.5). Of these trials, 315 were grouped over the 5 conditions, and 63 trials were used for familiarization or washout. First, 18 familiarization trials (F) were performed. Subsequently, all subjects performed the baseline1 (B1) of 63 trials without any damping forces. After B1, subjects performed, in random order, three conditions C1, C2 and C3, each of 63 trials. Between each condition, 18 washout trials (W1, W2 and W3) were performed to reduce learning carryover from one condition to the next. After W3, subjects performed a second baseline (B2) of 63 trials.

Target reaching distance $D$ was chosen to lie within the reachable workspace of the human arm and the used manipulandum, and to have a large spread, with

Table 6.1: Used distances ( $D$ in $\mathrm{cm}$ ), and index of difficulty ( $I D$ in bits) result in nine combinations of target size $(W$ in $\mathrm{cm})$ per frame.

\begin{tabular}{llll}
\hline & \multicolumn{3}{c}{ ID [bits] } \\
\cline { 2 - 4 }$D[\mathrm{~cm}]$ & 3.5 & 4.5 & 5.5 \\
\hline 18 & 1.75 & 0.83 & 0.41 \\
23 & 2.23 & 1.06 & 0.52 \\
28 & 2.71 & 1.29 & 0.63 \\
\hline
\end{tabular}




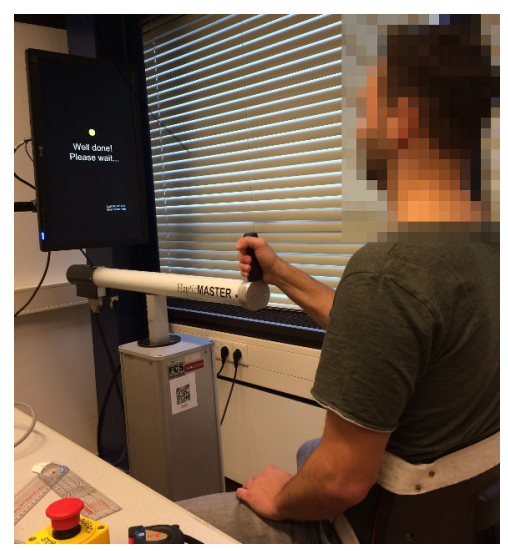

Figure 6.3: A subject uses the Moog HapticMaster to move a cursor to a target shown on screen, while experiencing different kinds of damping forces.

reasonable target sizes. The chosen $I D$ values were based on the fact that below $I D=3.5$ movements are ballistic and possibly without visual feedback, although literature disagrees on whether this happens at this ID value. Above $I D=5.5$ the ID makes the targets too small to assume Fitts' law is valid for this experiment when showing the target on screen $[169,170]$. We chose three different $I D$ values $(3.5,4.5$ and 5.5 bits) to be able to better observe linearity when fitting the data with (6.1).

Combinations of three values for $D$ and three values for ID give nine values for $W$ according to (6.2), as shown in Table 6.1. These nine combinations, which together we call a 'frame', were presented in random order. Washout and familiarization blocks consisted of two frames, while the five conditions consisted of seven frames.

\subsubsection{Subject Instruction}

Subjects were instructed to move a cursor (which was set to emulate $12.5 \mathrm{~kg}$, unknown to the subject) from a starting position to a target position in front of them using a manipulandum (see Sec. 6.3.5). This reaching motion was done at shoulder height, in front of the sternum while moving straight forward.

The cursor and target were represented as a yellow and magenta circle respectively on a computer screen. The subjects had to perform the task as fast and accurately as possible and stay for $500 \mathrm{~ms}$ (dwell time) inside the target. Sub- 
jects were motivated to minimize their reaching time by an on-screen score that was calculated from the movement time and ID.

At the start of a trial, the text 'Go!' would appear on screen after a random amount of time. This strategy was chosen to avoid anticipation of this starting event. Then the subject moved the handle to the target on screen. If the subject would push the handle before the starting event, the screen would turn red, the manipulandum would not move, and the random count-down would restart. After the subject stayed in the target for the required dwell time, the manipulandum would automatically move back to a fixed 'home' position and stay there until the start of the next trial.

Subjects were told when a wash-out or change of condition would happen, and they could take breaks before washouts and after B1.

\subsubsection{Participants}

A total of 18 age matched healthy right-handed male subjects aged $24.4 \pm 2.8$ (mean \pm sd) years of age participated in this study. They were unaware of the aim of the experiment.

The Ethics Committee of the Vrije Universiteit of Amsterdam approved the study design, protocols and procedures, and informed consent was obtained from each subject.

\subsubsection{Set-up}

The used experimental set-up is shown in Fig. 6.3. Subjects were fixated in a chair by velcro straps to avoid compensatory trunk and shoulder movements. The one dimensional reaching movements are made on a Moog HapticMaster: an admittance controlled haptic device to emulate inertia and damping forces [171]. A computer monitor at a distance of approximately 1.5 meters from the subject showed the cursor, a target and instructive or motivational text (e.g. 'Please Wait'). The image size on screen was scaled to match the real-world movement distances and target sizes. The HapticMaster communicated with a Windows 10 operated laptop, over TCP/IP at a sample frequency between 800 $\mathrm{Hz}$ and $1000 \mathrm{~Hz}$. This communication was used to set damper parameters and log relevant data. This software, which included the visualization, was created in Visual Studio C 2013 Community (Microsoft, 2013). 


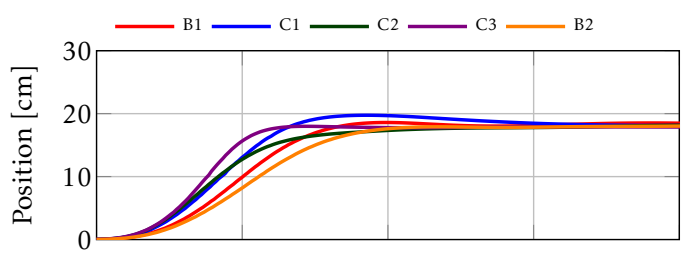

(a)

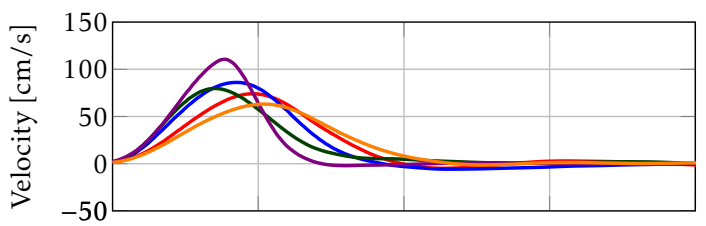

(b)

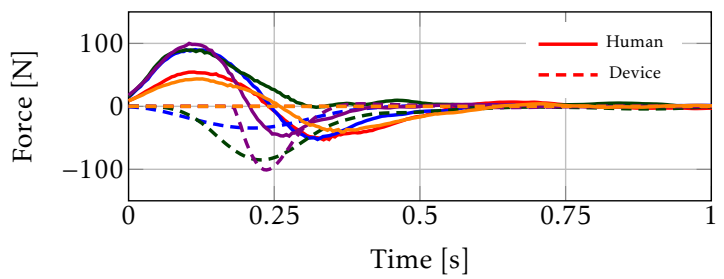

(c)

Figure 6.4: Typical reaching movements from a single subject, moving to a target at $D=18$ $\mathrm{cm}$, with $I D=5.5$ bits, within the last frame of all conditions. a) Position b) Velocity c) Force data from the human (solid) and the damper (dashed).

The positioning of the subject was defined such that for the largest $D$, the elbow extension was at most $140^{\circ}$. This avoided the use of the singularity of the arm to stop the reaching motion.

Safety of the subjects was guaranteed by proper positioning of the device such that they could never be hit or obstructed by it. Subjects could let go off the handle at any time. The device was equipped with an emergency stop button that could be pressed by either the researcher or the subject. 
B1

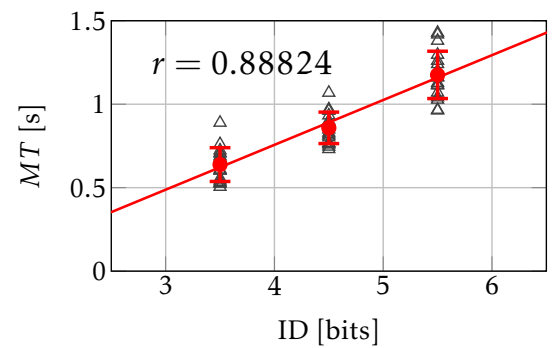

(a)

C2

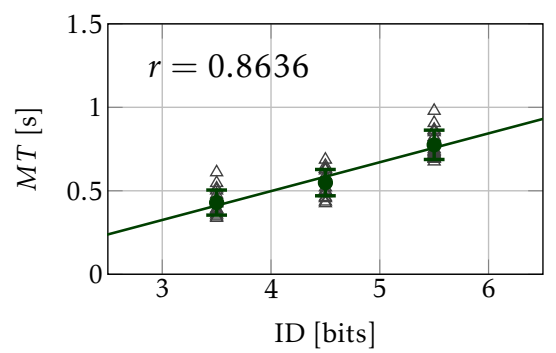

(c)

B2

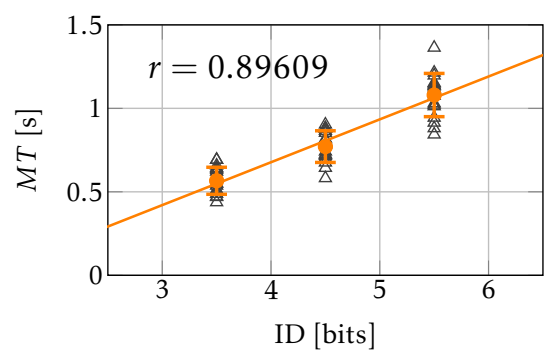

(e)
C1

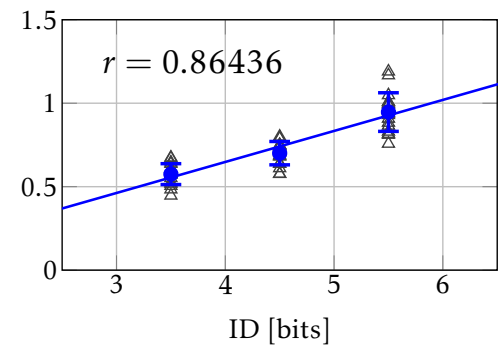

(b)

C3

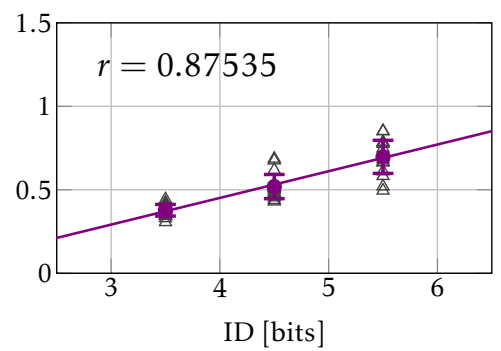

(d)

\section{Comparison}

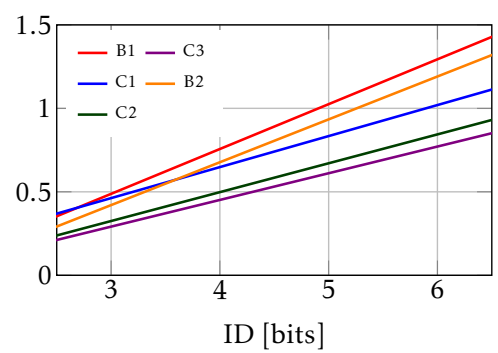

(f)

Figure 6.5: Linear regression of $I D$ in bits versus movement time $M T$ in s. a-e) Regression of conditions B1, C1, C2, C3 and B2. The mean per subject for that $I D$ and condition are shown as grey triangles. The mean over all 18 subjects is shown as a solid circle. The error bars indicate 1 standard deviation from the mean in both directions. f) Comparison of regressions for all conditions, showing lower slope $(b)$ and overall lower MT for conditions C2 and C3, compared to the other conditions. 


\subsubsection{Performance Metrics and Data Analysis}

Movement performance was evaluated in terms of movement time $(M T)$, mean human acceleration force during a trial, mean human deceleration force during a trial, and mean damper force during a trial. The $M T$ was determined a posteriori by 1) taking as the start of the movement the time instant when the subject would move faster than $2 \%$ of his maximal velocity during that trial (to remove reaction time offset) $[172,173], 2)$ removing the dwell time of $500 \mathrm{~ms}$ at the end of the movement time $[174,175]$.

Task accuracy was determined by counting the number of times the cursor reentered the target before the trial was accepted. A value higher than zero indicated that the cursor had exited the target and had to be moved back.

Since we are interested in steady-state performance only, we analyzed the last 27 trials (the last three frames) per condition. We ignore the first 36 trials, and assumed it would take that long to reach a performance plateau. This assumption was based on a pilot study performed with 12 subjects. Additionally, this assumption has been validated in the current study, as shown in Fig. 6.7.

Statistical analyses were performed between conditions, for each ID, and to determine whether a performance plateau was reached. Measurement data was fitted to Fitts' law (6.1) and (6.2) for each condition, using a linear least squares method. Familiarization and washout blocks were not analyzed.

As the data for all metrics was not normally distributed, the most appropriate statistical test was a Friedman test with a post-hoc Wilcoxon signed-rank test with Bonferroni correction on the significance threshold for 10 pair-wise comparisons.

\subsection{RESULTS}

\subsubsection{Typical Movement Profiles}

Figure 6.4 shows typical reaching movements from a single subject, moving to a target at $D=18 \mathrm{~cm}$, with $I D=5.5$ bits, within the last frame of all conditions. It is noted that all damping conditions (C1, C2 and C3) have a steeper slope (i.e. higher velocity) than the baselines (B1 and B2). Furthermore, for baseline movements the human force profile is very symmetrical, although this is not the case for the damping conditions (especially not for $\mathrm{C} 2$ ). 


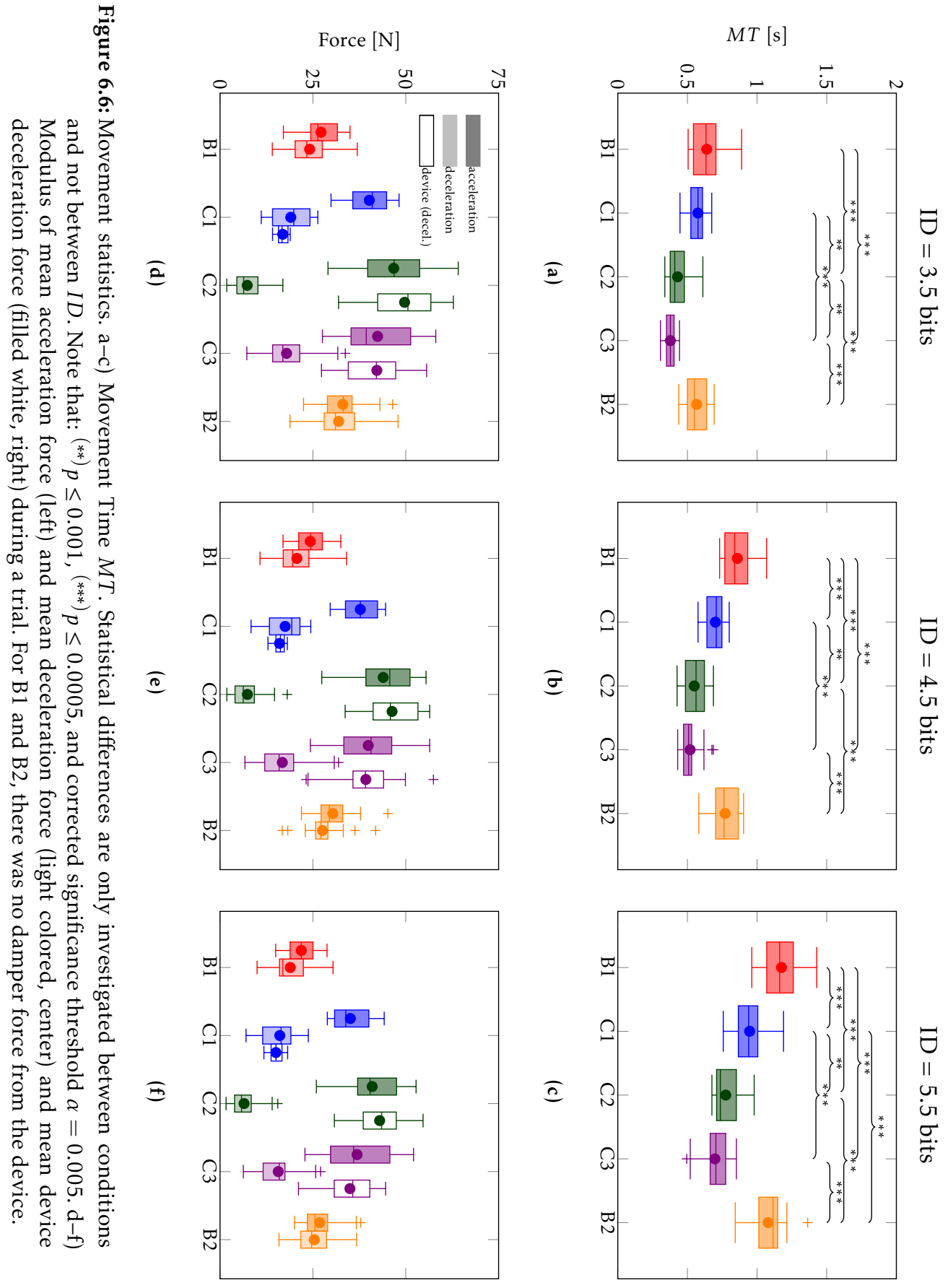




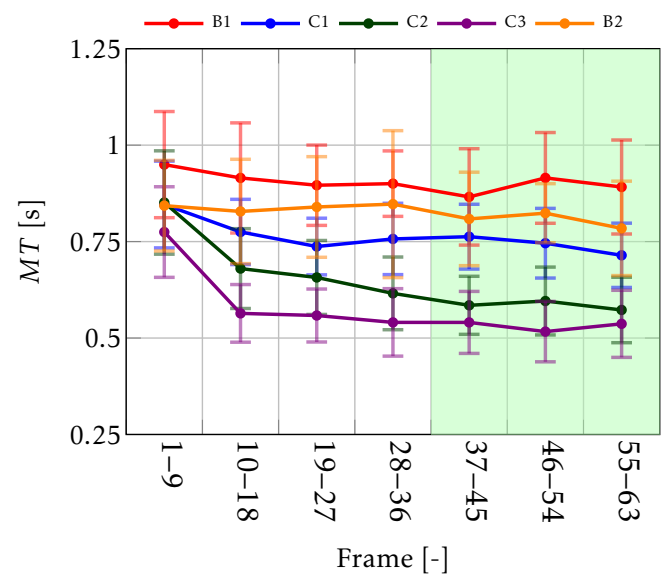

Figure 6.7: Learning curves showing the mean $M T$ per frame. The shaded green area highlights the last three frames that are taken for data analysis. A performance plateau is assumed to be reached in the last three frames. The error bars indicate one standard deviation from the mean.

\subsubsection{Relationship between ID and MT}

The ID and MT showed a linear relationship for all conditions (see Table 6.2 and Fig. 6.5). Using only the mean value per subject (averaging 9 movements per $I D$ per condition into 1 value) resulted in regression coefficient $r_{m}$ around 0.875 . Taking all 9 values per subject, per $I D$, per condition lead to the same intercept and slope ( $a$ and $b$ in (6.1)) but lower regression coefficient $r_{a}$ around 0.55 .

Table 6.2: Regression parameters. Coefficient $r_{m}$ is the regression coefficient for taking the mean $M T$ per subject for that ID and condition. Coefficient $r_{a}$ is the regression coefficient for taking all MT from a subject for that ID and condition.

\begin{tabular}{lllll}
\hline & $a[\mathrm{~s}]$ & $b[\mathrm{~s} / \mathrm{bits}]$ & $r_{m}$ & $r_{a}$ \\
\hline $\mathrm{B} 1$ & -0.3180 & 0.2686 & 0.8882 & 0.6218 \\
$\mathrm{C} 1$ & -0.0952 & 0.1858 & 0.8644 & 0.5979 \\
$\mathrm{C} 2$ & -0.1938 & 0.1730 & 0.8636 & 0.6299 \\
$\mathrm{C} 3$ & -0.1881 & 0.1599 & 0.8753 & 0.5460 \\
$\mathrm{~B} 2$ & -0.3498 & 0.2568 & 0.8961 & 0.6304 \\
\hline
\end{tabular}




\subsubsection{Within Subject Comparisons}

\section{Movement Times}

For all $I D$ values, there was a significant change in movement time between condition, $\left(p<0.0005, \mathrm{nDOF}=4, \chi_{I D=3.5}^{2}=48.44, \chi_{I D=4.5}^{2}=50.04, \chi_{I D=5.5}^{2}=\right.$ 57.47). Post-hoc analysis (see Fig. 6.6a-c) showed that for $I D=3.5$ the mean $M T$ was significantly different for all conditions except for B1-B2, B1-C1 and C1-B2. For $I D=4.5$ the mean $M T$ was significantly different for all conditions except for B1-B2, C2-C3 and C1-B2. For $I D=5.5$ the mean $M T$ was significantly different for all conditions, except B1-B2 and C2-C3.

To investigate learning effects, we also took the mean $M T$ per frame, resulting in 7 values per condition, and averaged over these means for 18 subjects. This gave an overall 'learning curve', as shown in Fig. 6.7. For all conditions the MT did not change significantly in the last three frames. This result validates the assumption that a performance plateau was reached in the last 27 trials.

\section{Forces}

Mean human acceleration forces, and human and device deceleration forces are shown in Fig. 6.6d-f. For all ID values, there was a significant change in mean human acceleration force from the subject between conditions, $(p<0.0005, \mathrm{nDOF}=4$, $\left.\chi_{I D=3.5}^{2}=48.22, \chi_{I D=4.5}^{2}=53.29, \chi_{I D=5.5}^{2}=55.47\right)$. Mean human acceleration force was not significantly different (n.s.d) between C1-C3 and C2-C3 for all $I D$ s, and n.s.d. for $\mathrm{C} 1-\mathrm{C} 2$ for $I D=3.5$ bits. For all ID values, there was a significant change in mean human deceleration force from the subject versus condition, $\left(p<0.0005, \mathrm{nDOF}=4, \chi_{I D=3.5}^{2}=61.387, \chi_{I D=4.5}^{2}=56.22, \chi_{I D=5.5}^{2}=55.42\right)$. Mean human deceleration force was n.s.d. for B1-C3 and C1-C3 for all IDs, and n.s.d. for B1-C1 for $I D=4.5$ and 5.5 bits. For all $I D$ values, there was a significant change in maximal deceleration force form the device versus condition, $\left(p<0.0005, \mathrm{nDOF}=4, \chi_{I D=3.5}^{2}=28.78, \chi_{I D=4.5}^{2}=29.78, \chi_{I D=5.5}^{2}=32.44\right)$. Post-hoc analysis showed that all damping conditions had significantly different mean damping forces.

\section{Reaching Accuracy}

For all ID values, there was a significant change in reaching accuracy between conditions, $\left(p<0.0005, \mathrm{nDOF}=4, \chi_{I D=3.5}^{2}=51, \chi_{I D=4.5}^{2}=55.52, \chi_{I D=5.5}^{2}=\right.$ 
57.47). Post-hoc analysis showed that for all IDs there was no significant difference (n.s.d.) between C2-C3 and between B1-B2. For $I D=4.5$ there was n.s.d. between $\mathrm{C} 1-\mathrm{C} 3$ and for $I D=3.5$ there was n.s.d. between $\mathrm{C} 1-\mathrm{B} 2$. For $I D=3.5$ and $I D=4.5$ there was n.s.d. between B1-C2. All other pairwise comparisons were significantly different, $p \leq 0.001$.

For $I D=3.5$ the PDD C2 and C3 had an average of 0.04 target reentries, versus 0.40 for $\mathrm{B} 1$ and $\mathrm{B} 2$ and 0.29 for $\mathrm{C} 1$. For $I D=4.5$ the PDD C2 and C3 had an average of 0.19 target reentries, versus 0.69 for $\mathrm{B} 1$ and $\mathrm{B} 2$ and 0.48 for $\mathrm{C} 1$. For $I D=5.5$ the PDD C2 and C3 had an average of 0.42 extra target reentries, versus 1.3 for $\mathrm{B} 1$ and $\mathrm{B} 2$ and 0.9 for $\mathrm{C} 1$.

\subsection{Discussion}

\subsubsection{Metric Comparisons}

Using PDD to move $12.5 \mathrm{~kg}$ objects reduced movement times for almost all IDs by almost $40 \%$, compared to nondamping baselines. Constant damping also reduced reaching times, as expected from results presented in $[166,167,168]$, albeit not as much as PDD did. PDD also improved reaching accuracy. Subjects required fewer target reentries during PDD conditions than during baseline and constant damping conditions, with a reduction factor of almost 4 . PDD also resulted in a lower spread in movement times, compared to baselines and constant damping, resulting in a more consistent motion. Acceleration forces are higher for PDD than for constant damping and nondamping baselines, although human deceleration forces become lower.

\subsubsection{Movement Strategies}

Movement profiles for baseline movements mostly resemble minimum-jerk trajectories with bell-shaped symmetrical velocity profiles. These reaching movement trajectories have smooth accelerations and decelerations minimize endpoint error in the presence of signal-dependent noise [176]. The addition of PDD makes the velocity profile asymmetrical, with higher average human acceleration forces (increase of almost 100\%) in the acceleration phase of the movement, compared to baselines.

Human information processing proceeds by a series of essentially independent steps. In [177] it is suggested that two separate controlling processes are in- 
volved during reaching; first an acceleration phase and afterwards a fine-tuning phase. This could explain why PDD is successful in increasing accuracy (or for the same accuracy in reducing movement time), since it assists during the finetuning phase. This could also explain that constant damping hinders the acceleration phase, making $\mathrm{C} 1$ reaching times almost $30 \%$ slower than $\mathrm{C} 2$ and $\mathrm{C} 3$. For increasing $I D$, both acceleration and deceleration forces seem to decrease for all conditions. Possibly, a more cautious strategy is adopted when targets appear far away or are of smaller sizes.

To reduce reaching times with a passive system, higher forces are required for faster accelerations and decelerations. Since faster movements will have a higher mean and peak velocity, the expended net energy by the subject to perform this movement has increased. Condition $\mathrm{C} 2$ has the highest average acceleration forces (energy added) and lowest deceleration forces (energy extracted) by the subjects, out of all conditions. The energy added during condition C3 seems lower than for $\mathrm{C} 2$, and more energy is extracted by the subject. However, movement times were not significantly different between $\mathrm{C} 2$ and $\mathrm{C} 3$ for higher IDs.

\subsubsection{Fitts' Law}

Fitts' law seems to hold for PDD, when comparing regression coefficients with baseline. The found regression coefficients are slightly lower when compared to literature $(0.875$ versus 0.95$)$. In literature, different methods for determining the movement time in Fitts-like experiments are used; button pushes, tapping, the application of pressure on a surface or the usage of dwell time are common options. Compared to tapping and pressing, the usage of dwell time might have increased the timing spread, and therefore might have reduced the regression coefficient.

PDD and constant damping have faster movement times than no-damping baselines. The decrease in $M T$ between $\mathrm{B} 1$ and $\mathrm{B} 2$ conditions was minimal and not significant, from which we conclude no overall significant effect of learning over the complete experiment. For baseline and damper conditions the regression only differs in intercept $a$ (in s) and not in slope. The slope ( $b$ in s/bits) of Fitts' law is reciprocally related to the index of performance, or information transmission rate, during the reaching motion. For both baselines (B1 and B2) the slope is the same. The slope is also the same for all three damping conditions ( $\mathrm{C} 1, \mathrm{C} 2$ and $\mathrm{C} 3)$, but lower than baseline. From an information theoretical 
perspective, it is tempting to state that with any damping force more information was transferred per unit time to the subject to perform the motion. As discussed in [163], the fundamental interpretation of this information channel is not straightforward for human reaching. Future work will focus on investigating why this grouping in slope occurs.

\subsubsection{Position Dependency of the Damping Coefficient}

In this experiment, the d.c. was either constant (C1), or linearly increasing from different distances (C2 and C3). We picked the linearly increasing d.c., starting at different locations, to be able to control the influence of starting location (onset).

Different 'shapes' other than linear could have been used with later onset, monotonically increasing nonlinear, steeper and possibly exponential increase. If we would have used an exponentially increasing d.c., we cannot investigate the influence of onset versus shape; since it behaves like a d.c. that is zero over a long range and suddenly increases rapidly. Furthermore, a linearly increasing d.c. answers the current research questions properly.

From experience we know that rapidly increasing d.c. shapes work well, but too high gradient and too high value (e.g. a brick wall effect) reduces subjective likability. Optimizing the shape of the d.c. is an aspect we will look into in future work.

\subsection{CONCLUSION}

Results show that PDD reduced (compared to baseline and constant damping) movement times by more than $30 \%$ and reduced the number of target reentries, i.e. increasing reaching accuracy, by a factor of 4 . The results are inconclusive about whether the $18 \mathrm{~cm}$ ramp (C2) or $9 \mathrm{~cm}$ ramp (C3) in damping coefficient performs better in terms of accuracy and movement time. Force profiles hint at a marginally lower energy expenditure from the subjects in condition $\mathrm{C} 2$ than in C3. Both strategies exploit high accelerations during the acceleration phase, and assisted deceleration of the movement to reduce overall reaching time. Results indicate that PDD is a useful haptic force to get humans to decrease their reaching movement times, while increasing their targeting accuracy. 
Arvid Q.L. Keemink, Niek W.M. Beckers and Herman van der Kooij

Fast is Fine, but Accuracy is Final.

- Wyatt Earp, 1923

Abstract-In previous experiments we showed that subjects reaching in constant and position-dependent longitudinal damping fields were able to reduce their movement time (MT) and increase end-point accuracy. The MT versus movement distance and prescribed end-point accuracy agreed with Fitts' Law. The explanation to why subjects were able to have shorter MT while subjected to impeding damping forces is, however, not explained by Fitts' relationship. Based on the minimal variance principle of Harris and Wolpert, we propose that humans exploit the noise-filtering behavior of constant, or varying, damping forces. These damping forces attenuate mechanical effects of activation dependent motor noise. This allows for higher motor activation and shorter MT without losing end-point accuracy. Consequently, higher allowed motor activation allows for higher accelerations that lead to higher peak velocities, resulting in shorter reaching times. Linear and non-linear stochastic optimal control and optimal estimation models with multiplicative noise corroborate measurement data, supporting our hypothesis.

LIST OF USED SYMBOLS AND ABBREVIATIONS

\begin{tabular}{ll}
\hline Symbol & Explanation \\
\hline $\mathbf{A}(x)$ & discrete-time position dependent state transition matrix \\
${ }^{c} \mathbf{A}(x)$ & continuous-time position dependent state transition matrix \\
$b, b_{c}$ & constant linear damping coefficient
\end{tabular}

This chapter will be rewritten together with Chapter 6 to be submitted as a single journal publication. 


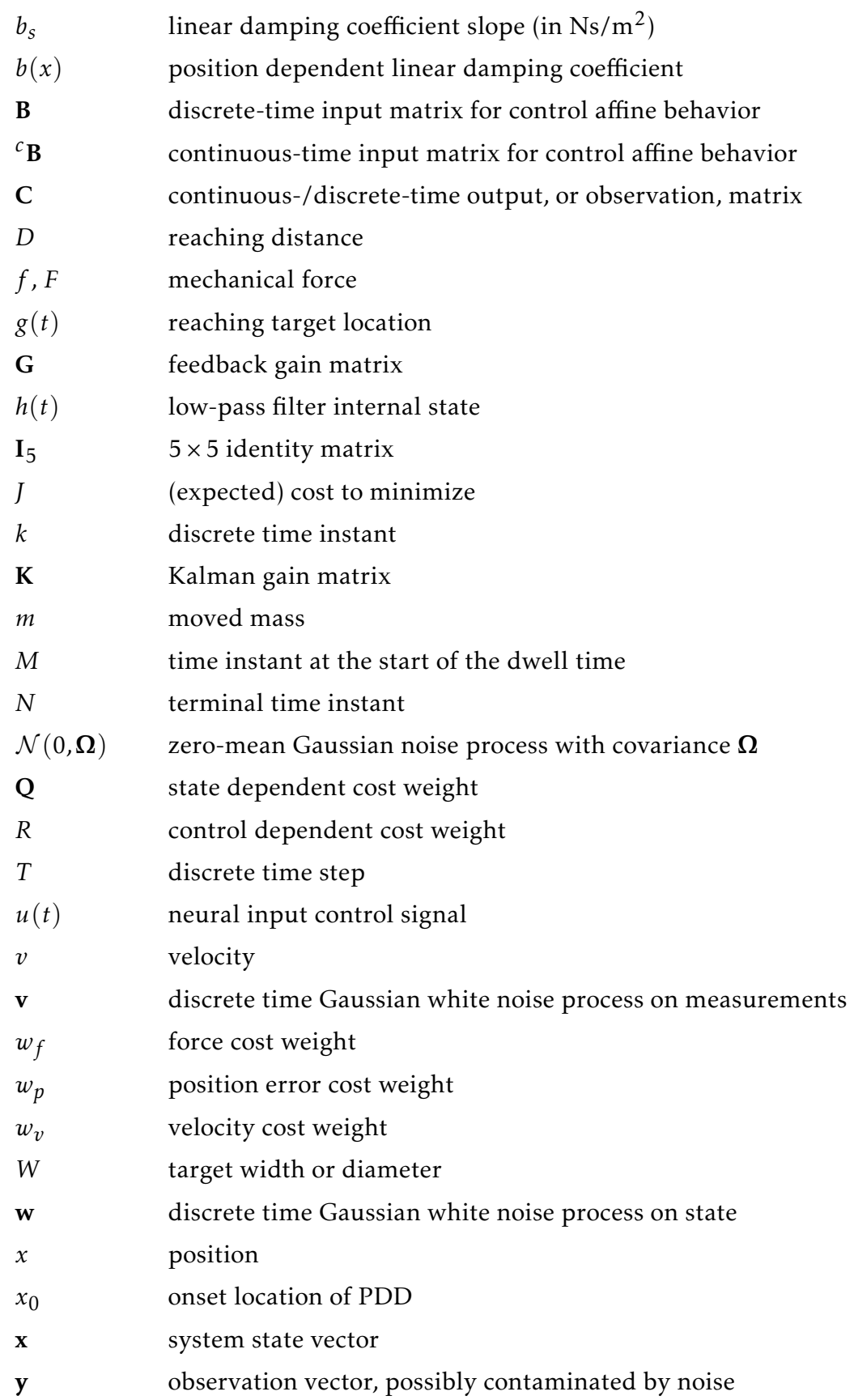




$\begin{array}{ll}\boldsymbol{\Gamma} & \text { multiplicative noise scaling matrix (size of } \mathbf{B}) \\ \xi & \text { multiplicative motor noise } \\ \sigma & \text { noise standard deviation } \\ \tau & \text { linear system time constant } \\ \Omega & \text { Gaussian noise covariance matrix }\end{array}$

\begin{tabular}{ll}
\hline Abbrev. & Explanation \\
\hline B & baseline; no damping movement \\
C1 & condition 1 ; constant damping coefficient of $40 \mathrm{Ns} / \mathrm{m}$ \\
C2 & condition 2; damping coefficient increasing over $18 \mathrm{~cm}$ from 0 to 200 \\
& Ns/m at the target \\
C3 & condition 3; damping coefficient increasing over $9 \mathrm{~cm}$ from 0 to 200 \\
& Ns/m at the target \\
CNS & central nervous system \\
iLQG & iterative linear quadratic Gaussian controller \\
LQG & linear quadratic Gaussian controller \\
LQG & linear quadratic Gaussian controller with multiplicative motor noise \\
MT & movement time \\
PDD & position dependent damping \\
\hline
\end{tabular}

\subsection{INTRODUCTION}

A human's mechanical power and force capabilities can be augmented by assistive haptic devices. Such devices amplify or complement the user's force, or display forces to steer and inform the user. Virtual fixtures [178], such as potential force fields, have been used as haptic force sources to assist humans in tasks in haptic tele-operation or during power augmentation. If there is any relevant intelligence in the modulation of location and impedance of these virtual fixtures, the haptic method can be regarded as haptic shared control [17]. Shared control uses haptic forces and change in device impedance to give cues, increase safety, assist movements or force the user to change movement strategy.

During haptic shared control different kinds of haptic forces can be provided to the user. Viscous damping forces are haptic forces that mimic a physical dis- 
sipative force $F$, proportional through a damping coefficient $b$ to the velocity of movement $v$ in the form $F=-b v$. In contrast to potential forces, like stiffness, damping forces are more 'forgiving' by allowing users to move against them, albeit at the cost of expending more energy and usually taking more task completion time. Furthermore, damping forces are passive in the sense that they can only dissipate energy from a mechanical system, making dissipative assistive devices inherently stabilized and therefore safe.

Constantly moving a device in a viscous damping field will unnecessarily increase energy expenditure of the user. Therefore, damping can be made position dependent (i.e. position dependent damping, or PDD [179], see Chapter 6) by having the damping coefficient change and increase near important task-related 'movement targets'. Because damping can be moved against without the device pushing the user back to a specific point, the task-related target location is not required to be known with high accuracy.

If the device consistently applies these damping forces around task-relevant locations on the user, (s)he will change their reaching movement plans accordingly. Motor adaptation research has mainly focused on orthogonal velocity dependent curl fields, but not on how humans adapt their movement strategy to longitudinal (i.e. opposing the movement in parallel to the movement direction) damping forces. Although orthogonal (curl) forces and longitudinal (damping) forces are both velocity dependent, the former is an active force (see similar reasoning of anti-symmetric stiffness in [99]) that steers the human reaching motion off-course, while the latter is a passive force that impedes the motion. Therefore, any conclusions drawn about curl-field adaptation cannot be directly applied to longitudinal damping.

Previous research into the influence of damping forces on reaching performance showed that reaching underwater increased reaching movement times (MT) $[164,165]$. The underwater environment is viscously dissipative, but highly inertial as well. Other research showed that constant static friction helped in reducing reaching times and increasing accuracy for moving low masses during reaching $[166,167,168]$. Crommentuijn et al. [168] found that increasing the Coulomb friction magnitude did not negatively impact performance but only decreased the likability of the task. The authors hypothesized that friction forces provide a predictable constant deceleration and act as a filter to unintentional movement. However, they did not investigate the influence of friction or damping forces when subjects move high masses. The authors also did not focus on how motion and force profiles changed after adaptation to the friction forces 
and how to explain or predict the steady-state change in human movement strategies.

Our previous research [179] (see Chapter 6) showed that constant damping and PDD both increased steady-state reaching performance in terms of MT and end-point accuracy, for moving masses of $12.5 \mathrm{~kg}$, which is much heavier than the average human arm. Fitts' Law regressions were useful in comparing results between conditions, but gave no insight into why constant damping and PDD resulted in better reaching performance compared to reaching without damping. Because of its accuracy, simplicity and utility, Fitts' Law is a widely used model for predicting movement time performance for rapid aimed arm movements, with a good fit to empirical data [180]. However, there is no satisfactory psychomotor theory that explains Fitts' law or why the coefficients change for changing dynamics, because this law only quantitatively explains how movement time relates to movement amplitude and target size.

In this work, we will focus on how users adapted their movement plan when subjected to these damping forces and attempt to explain steady-state performance results through a neuro-computational model that shows that damping attenuates mechanical effects of multiplicative motor noise.

\subsubsection{Motor Adaptation to Haptic Forces}

Literature shows that humans adapt and generalize their reaching strategies based on their own limb dynamics, state-dependent environment dynamics or haptic forces $[181,182,183,184,185,186]$. Evidence suggests that humans use two different internal models during coordinated reaching: a forward model (efferent copy of control through forward dynamics), using an internal representation of the body and environment to predict the body state [187], and an inverse model [188] to calculate the motor commands needed to perform a movement in a combined feed-forward and feedback controlled fashion. Although this explains how control is carried out, it does not explain the reason for motor adaptation to different environment dynamics.

It has been proposed that humans perform their motions in some optimal way. One of the first computational models for reaching was the 'minimumjerk' model proposed by Flash and Hogan [189]. According to this model humans change and optimize their motor plan to minimize integral of squared jerk, which is the time derivative of acceleration. However, others [190, 191, 162] argue that minimum-jerk cannot be the correct optimality criterion in general for 
arbitrary environment dynamics. There is no evidence that humans can properly measure jerk, and evolution of this optimization strategy is deemed to be highly unlikely [190]. The bell shape velocity profiles and apparent trajectory smoothness seen in reaching movements are hence due to some other, more fundamental, optimality principle.

Harris and Wolpert [190] proposed that the central nervous system (CNS) optimizes reaching by minimizing the deviation at the target position; called the minimum variance model. This model is based on the fact that the efferent and afferent signals in the human body are subjected to noise. They stated that the motor command signals are characterized by signal-dependent noise, or multiplicative motor noise. This means that higher muscles activation results in higher levels of muscle force noise. Their first-order estimation was that the standard deviation of this noise increases linearly with the mean force output. This signaldependent noise comes from the neural motor-command signals [192].

This movement uncertainty due to noise has to be optimally integrated in a movement plan to make an optimal movement. For each new environment, in our experiment the damping condition, the motor plan is reoptimized for those dynamics [191]. Therefore, a proposed model is the one of stochastic optimal control and optimal estimation [193, 194], that captures the signal-dependent noise, together with metabolic energy expenditure in a stochastic optimal control framework.

In this model the central nervous system needs to predict sensory consequences of our motor commands (using the forward model); then it needs to combine these predictions with delayed sensory information from proprioception and vision to infer the state of the body and environment (optimal state estimation); with this information the inverse model is used to tune the feedbackcontrol gains optimally to maximize some measure of performance (optimal control) [195].

Several mathematical stochastic optimal control and optimal estimation methods, known as LQG methods, have been put forth to mathematically model reaching movements, showing high agreement with empirical data [186, 194, $195,196]$.

\subsubsection{Hypothesis}

In this work attempt to explain why subjects were able to reduce their MT of reaching motions made when subjected to damping forces. Building on the min- 
imal variance principle and stochastic optimal control models, our hypothesis is that damping forces assist in reducing end-point variance introduced by both additive and multiplicative motor noise. Subjects have to guarantee an endpoint variance sufficient to perform and successfully finish the task. This requested end-point variance is not necessarily minimal, but sufficient for the requested target size. Therefore, subjects can apply high control forces that would lead to more end-point variance in the absence of (position dependent) damping. This, in result, leads to shorter movement times.

The attenuation of the noise effects cannot be reasoned about in an open-loop fashion as done in [190] and [197], because the effects of multiplicative motor noise that scales linearly with the control signal are also attenuated by closed loop feedback. It is a hard problem to predict the end-point variance of a closed loop system with time varying gains, as is the case for this stochastic optimal control problem. However, numerical simulations will shed light on the matter in Sec. 7.3.

Evidence that corroborates this hypothesis will be discussed in the following sections by applying stochastic optimal control and optimal estimation theory.

\subsection{DYNAMICS AND OPTIMAL CONTROL OF THE TASK}

The reaching movements and strategies observed in the experiments described in [179] are modeled in this section. For information on the subject instruction and experimental methods we refer to that same paper, or Chapter 6, Sec. 6.3. The reaching dynamics are modeled after Todorov [194], Diedrichsen [198] and Izawa [196]. We believe it is sufficient to neglect multi-link arm dynamics, and simplify the dynamics to a single degree of freedom point mass on which a damper acts. The human force is generated by the neural input signal $u(t)$ that passes through two first-order low-pass filters, both with time constant $\tau$, to model the activation dynamics and calcium dynamics of the muscle.

The continuous-time dynamics of this problem has five states $\mathbf{x}(t)=[x(t), \dot{x}(t)$, $f(t), h(t), g(t)]^{T}$, where $x(t)$ is the position of moved mass $m, \dot{x}(t)$ is the mass velocity, $f(t)$ is the force the human applies on the mass, $h(t)$ is the intermediate state of the low-pass filtered neural activation $u(t)$, and $g(t)$ is the goal location of the reaching movement that is chosen a priori and appended to the state vector. 


\subsubsection{State Equations}

The noise-free equations of motion of the aforementioned dynamics are given by

$$
\begin{aligned}
m \ddot{x}(t) & =-b(x) \dot{x}(t)+f(t) \\
\dot{f}(t) & =\frac{h(t)-f(t)}{\tau}, \dot{h}(t)=\frac{u(t)-h(t)}{\tau},
\end{aligned}
$$

where $u(t)$ is the neural activation of the force generating muscles. The state evolution equations are given by

$$
\begin{aligned}
{\left[\begin{array}{c}
\dot{x}(t) \\
\ddot{x}(t) \\
\dot{f}(t) \\
\dot{h}(t) \\
\dot{g}(t)
\end{array}\right] } & =\left[\begin{array}{ccccc}
0 & 1 & 0 & 0 & 0 \\
0 & -\frac{b(x)}{m} & \frac{1}{m} & 0 & 0 \\
0 & 0 & -\frac{1}{\tau} & \frac{1}{\tau} & 0 \\
0 & 0 & 0 & -\frac{1}{\tau} & 0 \\
0 & 0 & 0 & 0 & 0
\end{array}\right]\left[\begin{array}{c}
x(t) \\
\dot{x}(t) \\
f(t) \\
h(t) \\
g(t)
\end{array}\right]+\left[\begin{array}{l}
0 \\
0 \\
0 \\
\frac{1}{\tau} \\
0
\end{array}\right] u(t) \\
\dot{\mathbf{x}} & ={ }^{c} \mathbf{A}(x) \mathbf{x}(t)+{ }^{c} \mathbf{B} u(t) .
\end{aligned}
$$

The damping coefficient $b(x)$ is given by

$$
b(x)= \begin{cases}0, & \text { if no damping, } \\ b_{c}, & \text { if constant damping, } \\ b_{s} \max \left\{0, x(t)-x_{0}\right\}, & \text { if PDD, }\end{cases}
$$

with constant parameters: damping coefficient $b_{c}$, damping coefficient slope $b_{s}$ and positional offset $x_{0}$.

\subsubsection{Time Discretization}

The optimal control problem is in general more easily solved in discrete time for both linear and nonlinear dynamical systems. Therefore, the continuous-time 
dynamics captured in state evolution matrix ${ }^{c} \mathbf{A}(x)$ are discretized through forward Euler integration with timestep $T$, and time instant $k$, as

$$
\begin{aligned}
\mathbf{A}(x) & =\mathbf{I}_{5}+{ }^{c} \mathbf{A}(x) T \\
\mathbf{B} & ={ }^{c} \mathbf{B} T,
\end{aligned}
$$

where $\mathbf{I}_{5}$ is the $5 \times 5$ identity matrix. In contrast to $[198,196]$ we do not incorporate feedback delay in the disrectization, because we assume that these movements, due to their short durations, are effectively feed-forward controlled (even though the models might use an optimal feedback controller), and removed all response time from measurement data (see Chapter 6, Sec. 6.3.6).

\subsubsection{Adding Noise}

The system is augmented with zero-mean Gaussian noise sources $(\mathcal{N}(0, \boldsymbol{\Omega}))$ with specified covariance $\boldsymbol{\Omega}$. The relevant noise sources are:

- noise with unit variance that will be scaled by neural activation: $\xi_{k} \sim$ $\mathcal{N}(0,1)$,

- process noise $\mathbf{w}_{k} \sim \mathcal{N}\left(0, \Omega^{w}\right)$,

- measurement noise $\mathbf{v}_{k} \sim \mathcal{N}\left(0, \Omega^{v}\right)$ that acts on position, velocity and force state.

The discrete time dynamics with noise become

$$
\begin{aligned}
\mathbf{x}_{k+1} & =\mathbf{A}(x) \mathbf{x}_{k}+\left(\mathbf{B}+\boldsymbol{\Gamma} \xi_{k}\right) u_{k}+\mathbf{w}_{k} \\
\mathbf{y}_{k} & =\mathbf{C} \mathbf{x}_{k}+\mathbf{v}_{k} .
\end{aligned}
$$

Matrix C picks the states to be observed the CNS. Vector $\Gamma=\left[\begin{array}{lllll}0 & 0 & 0 & \sigma_{u} & 0\end{array}\right]^{T}$ scales the unit noise variance of $\xi_{k}$ and positions it in the proper state equation for $h(t)$ (as outlined in [194, 191]). Note that, due to time discretization, all the noise amplitudes have to be scaled by $\sqrt{T}$ to normalize the random walk process $[199,200]$.

\subsubsection{Optimal Control of the Task}

An optimal controller and estimator can be designed for the dynamics described in (7.1) lasting for time instants $k=1, \ldots, N$. The modeled task is a reaching 
movement where the hand starts at some initial position and moves to a target position $g_{k}$ in a pre-specified time interval $M$, and dwells inside the target until terminal time $N$ (this period of duration $N-M$ is the dwell period).

The optimal controller minimizes the expected cost

$$
J=E\left\{\mathbf{x}_{N}^{T} \mathbf{Q}_{N} \mathbf{x}_{N}+\sum_{k=1}^{N}\left(\mathbf{x}_{k}^{T} \mathbf{Q}_{k} \mathbf{x}_{k}+R_{k} u_{k}^{2}\right)\right\},
$$

with $E\{\cdot\}$ the expected value of a stochastic variable. Parameter $R_{k}>0$ denotes the relative cost on energy expenditure, which penalizes high control signals, and therefore influences the level of multiplicative motor noise. The state dependent cost can be given as $\mathbf{Q}_{k} \geq 0$ during the reaching movement, or as terminal cost $\mathbf{Q}_{N} \geq 0$. The terminal cost is held during the whole dwell period as $\mathbf{Q}_{d}=\mathbf{Q}_{N}$. To enforce the reaching movement towards target $g_{k}$ the cost matrix $\mathbf{Q}$ is comprised of

$$
\begin{aligned}
\mathbf{Q}_{k} & =\mathbf{0}_{5 \times 5}, \text { for } k=1, \ldots, M-1 \\
\mathbf{Q}_{d} & =\left[\begin{array}{ccccc}
w_{p} & 0 & 0 & 0 & -w_{p} \\
0 & w_{v} & 0 & 0 & 0 \\
0 & 0 & w_{f} & 0 & 0 \\
0 & 0 & 0 & 0 & 0 \\
-w_{p} & 0 & 0 & 0 & w_{p}
\end{array}\right] \text {, for } k=M, \ldots, N,
\end{aligned}
$$

where time indices $k=1, \ldots, N-1$ denote the movement time, and indices $k=$ $M, \ldots, N$ denote the dwell period where the subject had to keep the cursor in the target. Cost weights $w_{p}, w_{v}$ and $w_{v}$ relatively weight the contributions of positioning error, velocity and used force respectively.

\subsubsection{Finding the Optimal Controller}

The optimal controller for the problem described in the previous section is called a Linear Quadratic Gaussian (LQG) controller. The addition of multiplicative motor noise in control requires an extension to the typical LQG $[194,191]$, which we denote by $\mathrm{LQG}^{+}$. The used controller equations are derived from [194] and [191] for linear dynamics (when $b(x)$ is constant, $0 \mathrm{Ns} / \mathrm{m}$ or $40 \mathrm{Ns} / \mathrm{m}$ ). In 
the case of controlling non-linear dynamics (when $b(x)$ is not constant) the controller is called an iterative LQG (iLQG) controller $[199,200]$.

The optimal strategy for the CNS is to apply a linear negative feedback controller with time-varying gains $\mathbf{G}_{k}$ that uses the fully observed state or optimal state-estimate $\hat{\mathbf{x}}_{k}$, to observe the state of the limb. For linear systems the controller is of the form

$$
u_{k}=-\mathbf{G}_{k} \hat{\mathbf{x}}_{k} .
$$

For nonlinear systems an iterative improvement to control signal $u_{k}$ is made by solving a related $\mathrm{LQG}^{+}$-like problem for the linear approximation around the current working point [199]. This process is repeated until convergence of the found control signals and movement kinematics. The addition to the control signal is based on a feedback controller, but with an added open loop component. However, the sequential addition of these feedback and feed-forward contributions belonging to different linear approximations around a working point makes the optimal control signal for the nonlinear system one that has some complex relation to state and not in a linear feedback fashion as in (7.2).

\subsubsection{Optimal Estimation of Measured Signals}

The optimal state estimator that gives $\hat{\mathbf{x}}_{k}$ for LTI systems with multiplicative noise in control is derived in [194] for systems with multiplicative motor noise.It is given as

$$
\hat{\mathbf{x}}_{k+1}=\left(\mathbf{A}-\mathbf{B G}_{k}\right) \hat{\mathbf{x}}_{k}+\mathbf{K}_{k}\left(\mathbf{y}_{k}-\mathbf{C} \hat{\mathbf{x}}_{k}\right) .
$$

Note that this only holds for constant, state-independent $\mathbf{A}$. This optimal state estimator is a modified Kalman filter, with Kalman gain $\mathbf{K}_{k}$, that has different update rules for state value and error covariance, compared to a typical Kalman filter.

\subsection{COMPARISON OF RESULTS}

In this section we compare some of the results of the experiment with the model results. The model had fixed weight values of: $w_{p}=10^{7}, w_{v}=10^{5}, w_{p}=10^{2}$, $R_{k}=1(k=1, \ldots, N-1)$. The moved mass was $m=12.5 \mathrm{~kg}$, the filter time constant $\tau=40 \mathrm{~ms}$ [194] and the calculation time step $T=10 \mathrm{~ms}$. The constant 
damping coefficient for $\mathrm{C} 1$ was $b_{c}=40 \mathrm{Ns} / \mathrm{m}$. Slope offset distance was $x_{0}=$ $0.09 \mathrm{~m}$ for $\mathrm{C} 2$ and $x_{0}=0.18 \mathrm{~m}$ for $\mathrm{C} 3$. The slope of the damping coefficient was $b_{s}=20 / 9 \mathrm{kNs} / \mathrm{m}^{2}$ for $\mathrm{C} 2$ and $b_{s}=10 / 9 \mathrm{kNs} / \mathrm{m}^{2}$ for $\mathrm{C} 3$.

Due to the way the experiment was set up, we do not have empirical data about end-point variance. The only guarantee is that subjects reached the target with sufficient accuracy to finish the task. Therefore, the standard deviation of the noise sources used in the rest of the work are chosen to reflect a qualitative trend, without being able to fit to empirical data of motion variance.

Two models used to explain the empirically observed behavior:

1. LTI $\mathrm{LQG}^{+}$: This model solves for the optimal controller for a linear timeinvariant system with additive measurement noise, additive state transition noise and activation-dependent noise. A Kalman filter estimates the states of the system that are disturbed by measurement noise. This model is used to only investigate the difference in behavior between additive and multiplicative noise, without non-linear behavior influencing the results. This was chosen to more clearly observe the difference in influence between multiplicative and additive noise on end-point variance. The observation matrix is set to $\mathrm{C}=\left[\begin{array}{ll}\mathbf{I}_{3} & \mathbf{0}_{3 \times 2}\end{array}\right]$.

2. iLQG: This model solves for the approximately optimal controller for a dynamical system with nonlinear dynamics introduced by a position dependent damping value. This model is chosen to explain salient features of the motion and force profiles, and to show how damping reduces the end-point variance of reaching motions performed with that model. This model does not have an optimal estimator and is deemed fully observable and uses $\mathbf{C}=\mathbf{I}_{5}$, to reduce the complexity and calculation time.

\subsubsection{Motion and Force Profiles}

Fig. 7.1 shows three subjects performing reaching movements over $D=0.18 \mathrm{~m}$, $I D=3.5$ bits for all four relevant conditions. Salient features of the measured motion profiles include

- B and C1: the symmetrical bell-shaped velocity profiles. Force profiles are symmetrical in magnitude for $\mathrm{B}$, but asymmetrical for $\mathrm{C} 1$, since the damper helps with slowing down.

- C2: the velocity profile is slightly asymmetrical with a longer stretched tail in the deceleration phase. The relative moment in time of peak velocity 


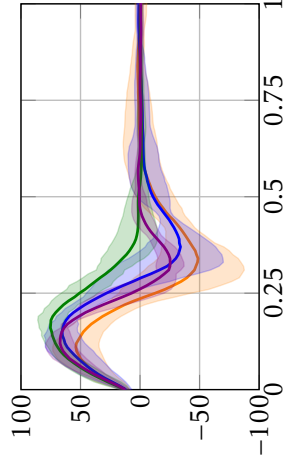

$[\mathrm{N}] \mathrm{\partial ว \lrcorner \textrm {O }}_{\mathrm{H}}$

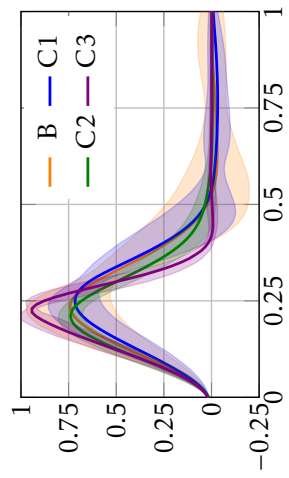

$[\mathrm{s} / \mathrm{w}] \cdot[\mathrm{\partial} \Lambda$

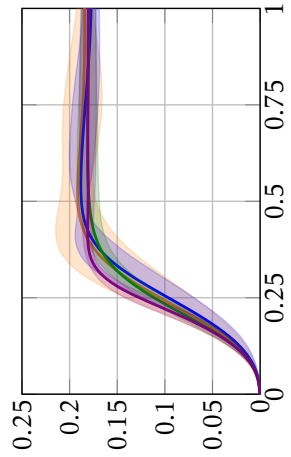

$[\mathrm{u}] \cdot \mathrm{so}_{\mathrm{d}}$

$: \angle$ toəlqns

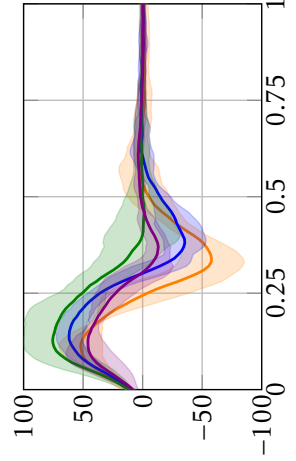

$[\mathrm{N}]$ วั.о⿴囗十

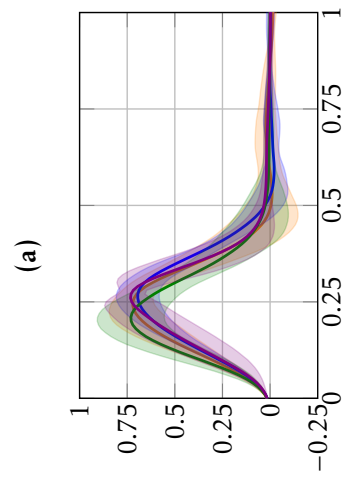

$[\mathrm{s} / \mathrm{u}] \cdot[\mathrm{\partial} \Lambda$

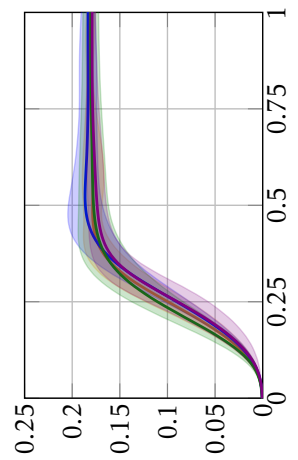

$[\mathrm{w}] \cdot \mathrm{so}_{\mathrm{d}}$

:t I poa!qns

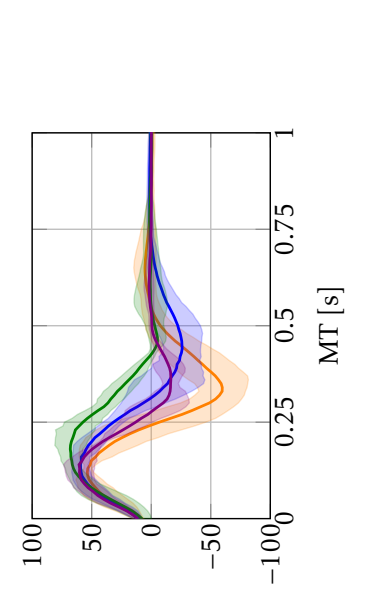

$\stackrel{\Xi}{\ddagger}$

$\Xi$

륭

$\stackrel{1}{8}$

$[\mathrm{N}]$ วั.о⿴囗十

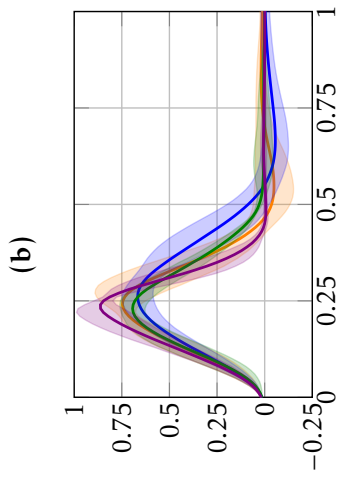

$[\mathrm{s} / \mathrm{u}] \cdot[\mathrm{\partial} \Lambda$

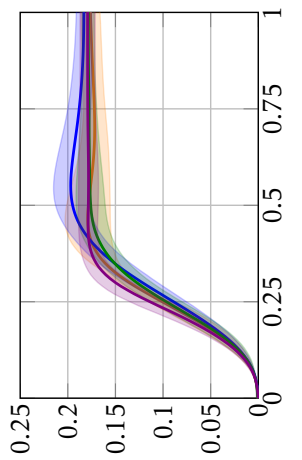

$[\mathrm{u}] \cdot \mathrm{so}_{\mathrm{d}}$ :8 I ¥oว!qns 


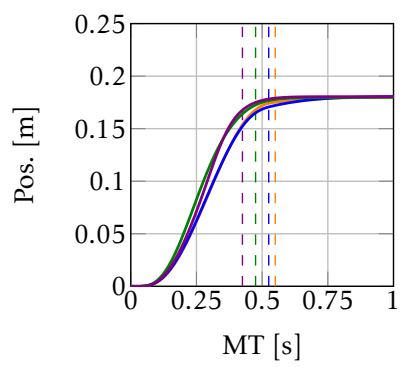

(a)

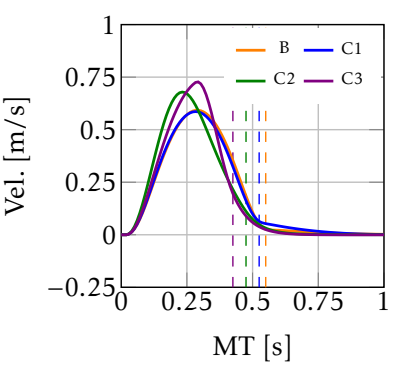

(b)

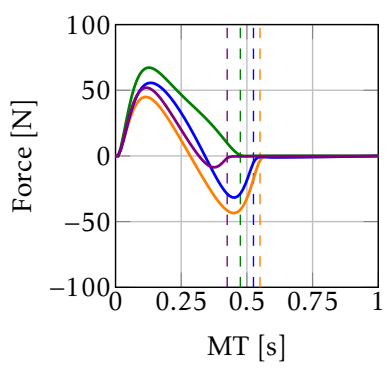

(c)

Figure 7.2: a) position profiles, b) velocity profiles, and c) force profiles produced by the iLQG method and the non-linear dynamical model. Dwell time starts at: 0.55 $\mathrm{s}$ for $\mathrm{B}, 0.525 \mathrm{~s}$ for $\mathrm{C} 1,0.475 \mathrm{~s}$ for $\mathrm{C} 2$ and at $0.425 \mathrm{~s}$ for $\mathrm{C} 3$, indicated with a colored dashed line. Notice the same asymmetry in the velocity profiles for C2 (longer right tail) and C3 (sharp drop-off on the right) as is visible in the experimental data in Fig. 7.1. The legend in b) applies to all subfigures.

is earlier, compared to B and C1. Furthermore, there is no need for the subjects to apply a deceleration force.

- C3: the velocity profile is asymmetrical, with a 'sharp bend' near the peak velocity with fast deceleration. The relative moment in time of peak velocity is later, close to the onset of the damping. Force profiles are asymmetrical in magnitude since the damper helps in slowing down.

Fig. 7.2 shows the outcomes of noise-free nonlinear models, solved with the iLQG method. All salient features shown in the experimental data are visible in the model results: the asymmetry in force profile magnitude, the asymmetry in velocity bell-shapes for $\mathrm{C} 2$ and $\mathrm{C} 3$. Although the first observation about force asymmetry might be thought of as a trivial result of complementing the damper force, the velocity and force profile time asymmetries are visible results from an optimization process. This comparison hints at the fact that reaching motions subjected to time-varying damping can be well explained and predicted by optimization methods such as optimal control. It shows that humans re-optimize their movement plan for the different dynamical conditions. 


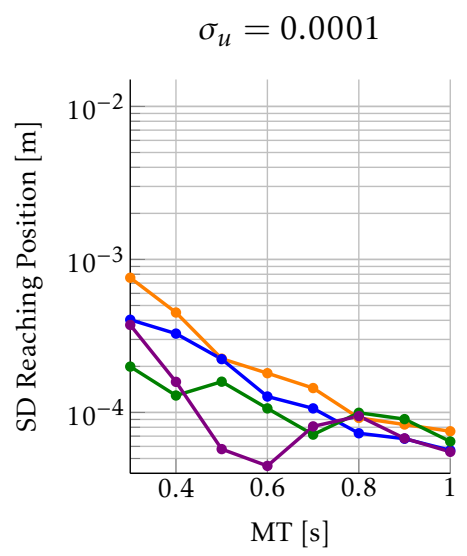

(a)

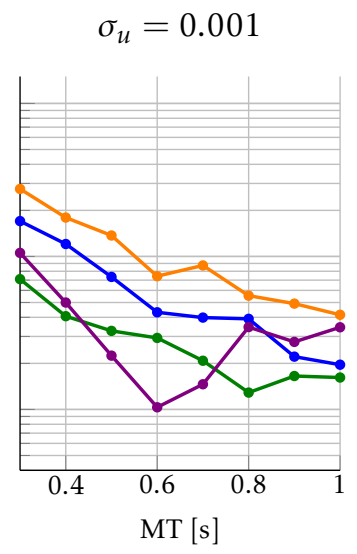

(b)

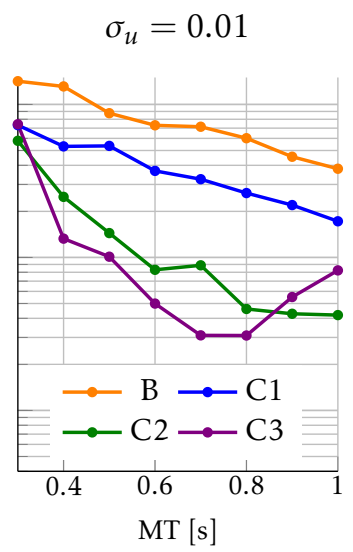

(c)

Figure 7.3: a-c) For different multiplicative noise scaling factors $\sigma_{u}$ : the standard deviation in reached end-point position taken from 40 movement repetitions of the iLQG method and the non-linear dynamical model at the end of the dwell period, for different MT. Reaching distance was $18 \mathrm{~cm}$. The legend in c) applies to all subfigures.

\subsubsection{Influence of Noise Variance}

\section{Multiplicative Noise and Fitts' Law}

The nonlinear dynamical model was run with activation-dependent noise with three different unitless noise standard deviation scaling values $\sigma_{u}=0.0001$, 0.001 and 0.01 in (7.1). The MT of the reaching motion was varied from 0.3 to $1.0 \mathrm{~s}$ with added dwell time of $0.5 \mathrm{~s}$. The model was run 40 times to get a measure of end-point accuracy in the form of standard deviation in reaching position.

The numerical results of these models are shown in Fig. 7.3. Fitt's Law behavior is observed in the increased accuracy, smaller end-point position standard deviation, for longer MT. Also, all damping conditions increase the model's endpoint accuracy. This might explain the reduced MT for conditions C1, C2 and C3 observed in the experiment. To achieve similar reaching accuracy for e.g. $\mathrm{C} 1$ as in B, we notice that this movement can be performed with less MT. This relative trend between conditions is true for all values of $\sigma_{u}$. 


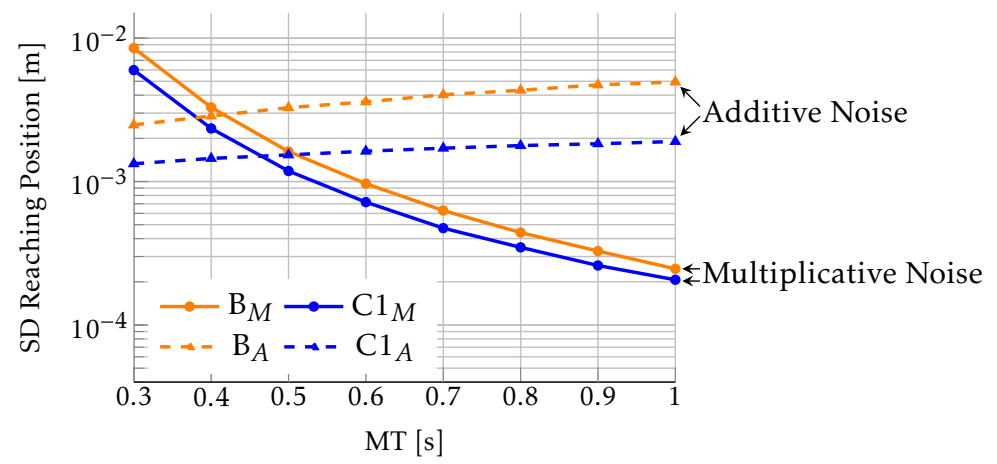

Figure 7.4: Additive process and measurement noise (subscript ' $A$ ' for additive, dashed lines) in the $\mathrm{LQG}^{+}$model shows that for larger MT the end-point variance increases. This is different from neural activation dependent noise (subscript ' $M$ ' for multiplicative, solid line). Damping forces filter contributions of all noise sources. The model results were averaged over 10,000 movement repetitions.

Notable is the fast decrease in variance for the C3 condition, with a minimal value at a MT of $0.6 \mathrm{~s}$. It is not known whether minimal variance would happen in real reaching tasks with that MT. Nevertheless, demanding a slow movement through an increasing damping field might require more neural activation for a longer time, which in turn actually reduces end-point accuracy.

\section{Additive Noise versus Multiplicative Noise}

To investigate the relative effect of multiplicative and additive noise on endpoint variability for LTI dynamics B and C1 only, the model was run with either a) multiplicative motor noise $\left(\sigma_{u}=0.3\right)$, or b) a combination of additive observation noise and process noise $\left(\sigma_{w}=5 \mathrm{~N}, \sigma_{v}=[0.025 \mathrm{~m}, 0.1 \mathrm{~m} / \mathrm{s}, 6.5 \mathrm{~N}]^{T}\right.$, the parameter values were chosen to deliver similar movement variance visually, as the observed variance during motions used to produce Fig. 7.3. The MT of the reaching motion was varied from 0.3 to $1.0 \mathrm{~s}$ with added dwell time of $0.5 \mathrm{~s}$. The model was run 10,000 times to get a measure of end-point accuracy in the form of standard deviation in reaching position.

The quantitative difference between the influence of additive and multiplicative noise (and therefore the exact used values for noise variance) are irrelevant in this example, because we are merely interested in the trend of the end-point variance versus MT and how this changes between conditions. The end-point 
variance increases with MT for additive noise, as is shown in Fig. 7.4. This trend is in contrast to the trend for dynamics with multiplicative motor noise alone. It would imply that taking a longer MT would result in less accurate movements. This is neither in accordance with Fitts' Law, nor with observation. Therefore, a model with large additive noise cannot be a model that describes the variance features of our reaching dynamics. Nevertheless, some amount of additive noise is unavoidable in the real motor system.

Added damping forces reduced end-point variance for both the multiplicative noise (this is a reconfirmation of the iLQG results for B and C1 in Fig. 7.3) as well as for additive noise. The human motor system is contaminated by more sources of noise than just a single multiplicative noise source. Damping forces might therefore also reduce contributing effects of other sources of noise.

\subsection{DISCUSSION AND FUTURE WORK}

The aim of this work was to give qualitative evidence from computational models to support the hypothesis that damping reduces end-point variance, allows for more motor activation and therefore reduces MT for fast reaching tasks [179].

Performing optimally controlled movements with the same cost function and same MT shows reaching kinematics with smaller end-point variance in conditions C1, C2 and C3. This corroborates results we presented in [179] (see Chapter 6). Salient features of motion and force profiles of the models are highly similar to experimental data. This is a strong indicator that the models used, and therefore also their response to noise, are an indicator of why subjects managed to reduce their reaching time. Damping force gives subjects the possibility to increase their neural activation and move faster, since it allows them to guarantee a certain targeting variance.

The experimental results, and model results agree with the hypothesis in [168] that the use of friction or damping, in general, shows a noise filtering behavior. Our used models give insight into how this filtering effect happens. The results and model agree with our hypothesis that MT can be reduced because subjects exploit the variance-reducing effect of the damping force, because the task instruction was to move as fast as possible.

Another way to think about the benefit of constant damping or PDD is that it acts as an antagonist muscle whose activation is noise-free and does not contribute to increasing the end-point variance. If this virtual antagonist muscle is 
'tuned properly' to activate during a required deceleration or targeting phase, it benefits the subject.

\subsubsection{Alternative View and Possible Falsification}

A second possible explanation, somewhat in line with the previous statement, of the decrease in MT for the damping conditions could be ascribed to implicit role distribution of the subject with the damping field. One could suggest that the subject considered the damping field as another 'agent' which aided the subject in te reaching movement by assisting in the deceleration phase of the movement. Reed et al. [201] showed that two human partners working together on a target acquisition task performed the task faster than individuals performing the same task. Through the recorded interaction forces, they observed specialization, in which one partner accelerated and the other partner decelerated the movement. The specialization was hypothesized to be the cause of the faster reaching movement [201]. However, the authors could not explain a mechanism behind the benefit of this role distribution.

To investigate this hypothesis, multiplicative force noise could be added to the damping force, i.e. noise that scales with the velocity state. According to our hypothesis, this should in turn increase MT. If this does not happen, however, it might be more indicative of benefits of human-human role distribution. Although this does not explain why this role distribution, with two agents that are both influenced by their own multiplicative motor noise, would result in a shorter MT.

\subsubsection{Model and Method Limitations}

The used optimal control reaching models do have their limitations and drawbacks. For physical reaching movements, the MT is an outcome metric of the performed actions. However, in the model, the MT is a predefined parameter. A similar issue is raised in [202] where they ascribe slower movements of Parkinson's disease patients to higher cost on energy. The high cost on energy would henceforth increase MT. However, a model with fixed MT does not show this behavior with different cost on energy expenditure.

This problem can be overcome by adding a non-zero state-dependent cost during the reaching motion before the dwell period. However, this would put more cost on being far away from the target, and less cost on being close. This is dif- 
ferent from the more reasonable idea of only inducing cost when not ending up at the target. Also, state dependent cost during reaching results in highly asymmetrical velocity and force profiles that are not observed in human reaching. However, cost during a dwell period or at terminal time does resemble human reaching kinematics very well.

For a Fitts'-like reaching task the state dependent cost could be made something highly nonlinear, similar to $(|D-x(t)|<W / 2)+$ energy cost, with $D$ and $W$ the reaching distance and target diameter respectively. The Boolean expression on the left would force the arm to reach the target with the demanded accuracy without differently penalizing distance from the target. Reweighting the energy cost would directly influence the found MT. However, such a non-linear cost has no proper derivative in state, and is therefore hard to apply in the iLQG setting where the non-linear cost is approximated by a quadratic cost in the linearization. Different solution strategies, like evolutionary optimization methods to find a parameterized control signal might be able to handle such a cost function better than the current methods.

The results of both the experiment and the model are also limited in that they cannot be extrapolated to arbitrary weights and arbitrary damping force magnitude. A more realistic muscle model with force saturation would be a straightforward extension to identify how humans would re-optimize their reaching strategy for interacting with such heavy mechanical mass or high damping force that they require peak voluntary muscle contraction.

\subsubsection{Experimental Limitations and Recommendations}

The currently performed experiment was set up mainly to determine how subjects' MT would change for all the damping conditions, compared to a no damping condition. The used protocol was not ideal to extract the information to explain the applied strategies with optimal control. There was no comparable measure of end-point accuracy to compare to the model results.

A Fitts' like reaching experiment that demands subjects to dwell in the target introduces more variance in MT than in tapping tasks [179]. A dwell time measurement also eliminates a proper measure of end-point variance. In future research, a more typical two-way tapping task or button-press task, but with high mass, might serve two useful purposes: 1) giving a proper measure of endpoint variance, 2) give a better fit to Fitts' law. 
Future research might focus more on single distance reaching with e.g. only two target sizes to remove any variance due to target distance and target size. All non-baseline conditions could have constant damping, with smaller steps in value between conditions. This might give more insight in how subjects reoptimize their movement, and (with a better cost on state, or terminal state) possibly how it can be explained with a relative reweighting between targeting error and energy expenditure. 

Part IV

GENERAL CONCLUSIONS 



\section{DISCUSSION}

This dissertation presented several studies towards methods to improve exoskeleton and interaction devices that physically support humans. The following three questions guided the research process:

\section{Research Questions}

I. How to support the full range of motion of the human shoulder?

II. How to implement stable admittance control with inertia reduction?

III. How do humans respond to dissipative shared control forces?

In this chapter the work presented in this dissertation will be reiterated and discussed, especially focusing on scientific contribution and context, other possible applications, limitations of the presented work and possible future development and research directions.

\subsection{SUPPORTING THE RANGE OF MOTION OF THE HUMAN SHOULDER}

Chapter 2 presented a method to visualize the range of motion (ROM) of the three degrees of freedom (DOF) of rotations of the human glenohumeral $(\mathrm{GH})$ joint. The visualization allows us to show the three DOF on a two-dimensional plane for easier communication and publication of results.

The method allows for a visual comparison of ROM between humans, healthy subjects and patients, between a human and a device (like an exoskeleton) or between devices. Colors were used to show kinematic conditioning of exoskeletons, although it is possible to show other parameters such as device inertia, applied forces, movement time, movement accuracy or translations of the shoulder girdle by varying colors, varying circle radii and multiple circles.

Due to the movement of the scapula at the scapulothoracic joint, and the clavicle at the sternoclavicular and acromioclavicular joint, the shoulder can also translate by performing up and downward motions (elevation and depression), 
and forward and backward motions (protraction and retraction). The presented work focuses solely on pure rotations of the GH joint, neglecting these translational motions. Nonetheless, the method could be expanded to show these two extra DOF in translation as arrows or lines in the circle centers, for example.

\section{Scientific Contribution and Context}

Compared to other methods to present shoulder ROM in literature, our method is able to show how the ROM is coupled between different movement directions in a single non-ambiguous 2D figure. In literature, the shoulder ROM is mostly presented along along the five medical directions of motion alone. This results in a workspace that is not representative of the actual human workspace; making the workspace too small in some configurations and too large in others. This is a serious problem during assistive device design and device evaluation where devices cannot exceed human ROM due to safety reasons.

Presenting merely the ROM of five medical directions also limits the communication of proper ROM during evaluation of how human shoulder ROM is affected by different movement pathologies.

Compared to projected 3D visualizations of the elbow-tip, our method shows axial rotation readily and does not suffer from projection ambiguity.

Our method also allows to show and compare kinematic, dynamic of performance metrics of humans and devices directly and intuitively, something that was not possible with other methods.

\section{Further Application}

The visualization method is not limited to the rotational ROM of the GH joint, but could be used for other three DOF rotation joints. Candidates for such display are the hip joint, the ankle joint and the complex combinations of joints in the wrist and elbow that provide the wrist with effectively three DOF.

Furthermore, the visualization method is useful to display the rotational ROM of non-anthropomorphic robots and mechanisms in general. This is commonly still shown as a (multi-)projected ambiguous 3D image of a workspace boundary, losing information about coupling within ROM.

\section{Limitations}

A minor drawback of the presented method is the required quantization of $\mathrm{GH}$ horizontal and GH elevation rotation, to avoid visual overlap between the circles 
of GH axial ROM. However, the grid can be made as finely spaced as preferred, or can be made non-uniform if specifically interesting areas of interest need to be shown.

The ROM presented in Fig. 2.1 is large. This might be influenced by inaccurate measurements done with a goniometer. Work was done [203], albeit not presented in this dissertation, where we measured the shoulder ROM with two inertial measurement units; one on the sternum and one on an elbow that was fixed in a 90 degrees flexion angle. This allowed for the measurement of relative rotation between the upper body and the upper arm, which equals the shoulder rotation. The subject would 'point' his/her elbow to a point on the grid and then traverse the complete axial-rotation ROM. This process was repeated for all grid intersections. Different measurement protocols were evaluated that build complete ROM diagrams similar to those in Chapter 2. This method showed a smaller ROM than in 2.1, especially in axial GH-rotation and horizontal GHrotation. During this work we noticed that measuring the ROM of the shoulder for a visualization as presented in Chapter 2 is intensive, and somewhat painful, even for healthy subjects. For patients with shoulder pain or limb weakness, intensive measurements that map the complete ROM like done here might be unfeasible. However, the problem of painful ROM measurement is not unique to this specific measurement and visualization method.

\section{Future Research}

Future work includes the quantitative evaluation of several case studies where ROM of the human shoulder and devices is presented in the new method and along the five medical directions of motion. In this way it is possible to get an expert opinion on using the method to communicate ROM and device properties. Furthermore, it is possible to quantify how much error is introduced by presenting the range of motion along the five medical directions, compared to presenting it in our new method.

Future work should also include an investigation into whether the history of motion influences the measured ROM in certain upper arm configuration.

Chapter 3 presented a conceptual kinematic design for a four DOF (or 4R) shoulder exoskeleton where the exoskeleton axes are non-orthogonal (i.e. if a more dominant joint rotates, the motion of the following joint axis describes an obtuse or acute cone, instead of a flat circle). Such a shoulder exoskeleton can be used to increase the ROM of conventional $3 \mathrm{R}$ exoskeletons by having more pos- 
sibilities to avoid collisions with the human body and avoid configurations with poor kinematic conditioning. Both of these problems limit the usable workspace current 3R exoskeleton designs.

The proposed $4 \mathrm{R}$ shoulder exoskeleton shoulder supports the GH rotations of the shoulder, while translations due to elevation, depression, protraction and retraction are taken care of by a separate translation mechanism that is placed in series, kinematically speaking in front of the joint, closer to the system's base.

We required an inverse kinematics (IK) solution method to calculate reference joint angles from non-prescribed motion trajectories. Such trajectories can be derived from real-time interaction control methods. Especially admittance control prescribes motions to an interaction device, such as an exoskeleton, after a user applies forces onto the device. Since these inputs are not known a-priori, a flexible solution strategy was needed.

\section{Scientific Contribution and Context}

We found that any $3 \mathrm{R}$ or $4 \mathrm{R}$ exoskeleton can support all 3D rotations (i.e. cover all of $S O(3)$ at least once) if and only if the sum of absolute differences between the angles-between-exoskeleton-axes and 90 degrees is smaller than, or equal to 90 degrees. This allows for a non-orthogonal joint design that can assist in avoiding body and internal collisions, compared to an orthogonal design, but achieve all possible rotations.

We chose to develop and evaluate a differential non-unique solution to the IK problem, instead of developing a direct unique 3DOF arm configuration to unique 4DOF joint-configuration mapping (i.e. a non-differential angle-to-angle mapping). The main reason for this is that for an angle-to-angle mapping the method becomes a look-up-table that needs to interpolate 3D rotations between arm configurations. Furthermore, such a method does not allow the exoskeleton to move to a configuration further away from the body and from singularity when the person inside the exoskeleton is not moving their arm.

Because the exoskeleton joint is redundant by one degree of freedom, a one dimensional self-motion-manifold (SMM) allows for internal motions of the joint that do not influence the motion or configuration of the human arm. Our method exploits this self-motion to continuously steer away from exoskeleton singularity and body collisions, as is shown for a specific conceptual exoskeleton design as a test case.

To guarantee that the exoskeleton joint would always steer away form the body and singularity fast enough, given some limit on input speed, a new method 
is used to converge along the SMM to a 'better' configuration. This method overcomes issues found in gradient projection of secondary movement objectives, which can get stuck in a local minimum. However, for sufficiently slow movements, and careful analysis, a gradient projection might be sufficient for a specific exoskeleton design. Furthermore, if a sufficiently complex gradient (or its potential) would be constructed, gradient projection and our new method would be identical. However, parameterizing a potential function to have a gradient that is always parallel and never perpendicular to the SMM is complicated.

The shown method assumed an instantaneous center of rotation inside the human GH joint. This is, however, not necessarily required. The methods also applies to an exoskeleton design where the axes of rotation do not intersect in the same point, or do not intersect at all. This could be the case if the fourth degree of freedom is parallel to the axial rotation of the upper arm, but with a translational offset. However, the collision constraints then become dependent on the fourth joint angle, which makes the analysis more involved.

The analysis method described in the chapter can also be used to develop inverse kinematics strategies for redundant designs with passive internal degrees of freedom. However, a solution might not always exist for any arbitrary design.

\section{Further Application}

The proposed method is also usable for generic $4 R$ joints that need to steer away from poor kinematic conditioning or collisions with the fixed world. A related problem could be the one of shoulder joints in humanoid or bio-inspired robots and hip-joints in exoskeletons and humanoid or bio-inspired robots.

\section{Limitations}

This research was mainly an investigation into whether a full range of motion exoskeleton is achievable in principle. An exoskeleton that can support the full human (shoulder) ROM is a luxury and usually not a necessity for many power augmentative tasks such as the support of healthy workers or support of rehabilitating patients. Especially the latter group might not benefit from a larger exoskeleton ROM, whereas the device complexity of the device and reliability on the software would be substantially increased. Enforcing end-of-ROM in such a device is crucial to not overextend the human (patient's) shoulder. This problem is challenging for more typical $3 \mathrm{R}$ designs, but becomes more difficult, and relies more on software safety, for $4 \mathrm{R}$ designs. However, we speculate that increasing 
the ROM of an exoskeleton to fit the human ROM increases user acceptance and could be especially useful for industrial, or agile military tasks.

\section{Future Research}

Future research should investigate possibilities and integration methods to perform the same method in the acceleration domain instead of the velocity domain. In that way the velocities can be made smooth, which is beneficial for joint motion tracking by low-level motion controllers.

Future work should also include better mechanical design than the proposed concentric arcs used in the presented conceptual prototype. Furthermore, a realistic solution has to be incorporated to place the fourth degree of rotation inside, or parallel to the upper arm. In a practical design there might not be a single center of rotation where all rotation axes intersect. This results in translations of the device, which demands possibly different joint-space constraints that the method should conform to.

\section{2 implementing Stable admittance Control With inertia Reduc- TION}

Chapter 4 presented admittance control as an interaction control paradigm. Admittance control requires a force sensor to measure the interaction forces between the human operator and the robot. This is commonly referred to as 'detecting the intent of the user' that lets the device know where to move. The measured forces are used as an input to a virtual dynamical model that calculates the desired robot motion state. A motion controller tries to control the robot motion to follow this desired state. In this way the robot can be programmed to behave like, and feel like, the virtual model.

Admittance control can be used to reduce inertia of heavy devices if the virtual model has a lower inertia than that of the real device. This is a relevant strategy for heavy lifting devices, as well as for rehabilitation and supportive robots. In all these cases the device or load is too heavy and too inert to move easily by the operator. In such cases admittance control acts as a method of power augmentation.

Compared to impedance controlled devices, feedback-only admittance control does not need models of the robot's parasitic effects, such as friction and parasitic dynamics, to simulate desired virtual dynamics. In practice, especially in- 
ertia reduction is more straightforward to perform on an admittance controlled device than on an impedance controlled device. An impedance controlled device would need to estimate or measure accelerations that result in noisy antiinertial forces. During admittance control, interaction force is measured that is passed through a model of virtual dynamics, that effectively filters noise contributions and allows for more smooth interaction. Furthermore, admittance control can almost trivially render stiffness and damping dynamics in a stable and passive way, because it is always motion controlled (see the analysis performed in the chapter). For example: simulating hard contact with admittance control is merely a change of motion state in the virtual model that the motion tracker has to follow, regardless of whether the device is inside or outside of that wall. Therefore, if the motion reference (the model) is not ejected from the wall, neither is the device. An impedance controller, however, generates a spring-damper force inside the wall, but no force outside it. Therefore a used device might be ejected out of the wall by that spring and bounce or shoot away when time quantization steps are too coarse, resulting in free floating, uncoupled, instability.

If the inertia of the robot is reduced by an admittance controller, the robot can become unstable when the force sensor comes in contact with, or is coupled to, a stiff environment. This is a relevant problem when a human operator holds and moves a device, or is attached to a device such as an exoskeleton. Possible muscle co-contraction and intrinsic limb properties introduce a non-zero amount of joint, limb and interface stiffness for the device to interact with.

This kind of coupled instability can be explained as non-passive, or active, behavior of the robot. The idea behind the energy passivity property of dynamical systems is that such systems cannot generate energy and are therefore stable during physical interaction with other passive systems. Any possible interconnection of passive systems is consequently also stable. If the admittance controlled device can be made to behave energetically passive, it is guaranteed to be stable when in contact with the (passive) human limb, and from everyday experience we realize that human interaction with passive objects is generally stable.

Imposing that a device behaves passively could be seen as being conservatively stable. Being passive implies interaction stability for an admittance controlled device coupled to infinite human stiffness, although the human's interaction stiffness in practical applications is limited and finite. Nonetheless, passivity conditions give relevant design goals that should be aimed for when aspiring interaction stability over a large range of device and environment impedance. 
Because if the preferred dynamics can be achieved while being passive and while compensating for disturbances, then both interaction stability and performance goals are achieved simultaneously.

\section{Scientific Contribution and Context}

Passivity conditions for controlled linear time-invariant systems are derived from the positive real condition (PRC), made popular by Colgate [92]. The PRC gives 'black box' frequency domain passivity conditions that put limits on the values of system and control parameters to guarantee that the admittance controlled robot behaves passively. The PRC, however, does not explain what causes the active behavior.

For admittance control with inertia reduction, and feedback motion control only, the PRC states that effectively no passive inertia reduction can be achieved. If attempted, there will always be some interaction stiffness of the human limb that will destabilize the interaction. Adding a constant amount of virtual damping allows for more integral velocity gain (i.e. more position control gain) to be used in the motion controller, allowing for a better low frequency approach of the intended lowered inertia. The amount of inertia that can be reduced in that way, however, is limited to few percent at most in any practical application.

The design guidelines we derive from the PRC all point in the same direction: 1) minimize phase lag between the measured force and resulting motion of the robot and 2) achieve as high motion control bandwidth as possible. The former limits any allowable low-pass filtering of force signals and puts limits on internal compliance of the robot. The latter tells us to use as much feed-forward (computed torque, or inverse dynamics) control, possible acceleration feedback or control, reduce loop-delay time and to use high sampling rates for the controller and updates of the virtual dynamics. Closed-loop feedback control is still required, however, to reject friction and other unmodeled disturbances that would have an otherwise parasitical effect on the felt robot impedance.

Even if the inertia of the robot is reduced towards the inertia of the virtual model, cuffs, handles or tools attached to the force sensor still introduce extra inertia and weight that is felt by both the force sensor and the user. This postsensor inertia can be compensated with acceleration feedback by feeding back a fake force signal that reduces the contribution of inertial forces on the device. However, this is a method of positive feedback, and the PRC rightfully states that this reduces the stability margins. 


\section{Further Application, Limitations and Future Research}

The guidelines presented can be used to design haptic simulation devices or tools for heavy lifting in industrial and health-care scenarios.

The work presented, however, is limited to linear single DOF robots. Furthermore, the post-sensor dynamics were assumed to be purely inertial. If the postsensor dynamics have a resonant mode or significant play, handling that behavior might be more difficult, even though its behavior is supposedly passive. Investigation is needed into whether different kinds of post-sensor dynamics result in different guidelines.

Future work would need to expand more on multi-DOF robots and robots with internal compliance. The work has to be more extensively compared with the pioneering work of Colgate [89] that states that any kind of internal compliance puts fundamental limits on the amount of passively achievable inertia reduction for any kind of controller.

Chapter 5 presented an analysis into the active nature of admittance control and developed a time-domain control method, called SPIS, to dissipate the energy that causes this instability.

\section{Scientific Contribution and Context}

The PRC is a black box condition that does not explain the source of active behavior in admittance control. A time-domain analysis shows that admittance control is not (strictly) passive due to energy created as a result from the power conjugation of velocity tracking error and the interaction force. This corroborates the idea that for admittance control the motion tracking should have high bandwidth, which would reduce velocity tracking error and in turn reduce the amount of generated energy.

From a system's perspective, an outer loop has to be closed to copy the measured force to the virtual dynamics, which is unilaterally connected to the motion controller. This unilateral connection, and the outer loop, break the 'energy consistent nature' of the control method as a whole. This results in unstable interaction of an admittance controlled robot with stiff environments, even without reduced stability margins due to time-discretization.

This shows that other methods proposed in literature (i.e. in $[145,146])$ cannot make admittance control with inertia reduction passive in the time-domain.

The apparent success of time-domain passivity enforcement on open-loop impedance controlled devices has been the fact that impedance controlled sys- 
tems are dynamically 'energetically consistent'. The virtual dynamics (commonly spring-damper combinations) are, albeit discrete-time, passive elements that fit in a physically consistent chain of systems that exchange energy. Instability then arises due to the time-discretization of these systems.

Dissipating the exact amount of energy generated with time-varying damper on the virtual model and the motion controller could also lead to passive behavior for admittance control with inertia reduction. However, this requires dynamic changes to, or read-outs of, a low level motion controller, which might not always available for motors with closed drive systems. Therefore, we attempted to achieve interaction stability by damping away excess energy from the virtual dynamics alone. However, because the virtual (desired) velocity from the virtual model can become arbitrarily small, it cannot be guaranteed to exactly dissipated the generated energy during each sample. Therefore, this method can only guarantee stability but not passivity.

The proposed SPIS method switches on a constant damper if the kinetic energy stored in the virtual model exceeds the energy introduced by the human operator. This stabilizes any unstable interaction. If the interaction was already passive, or if the device is free-floating, the damper does not need to intervene. Therefore the damper only switches on as a 'safety layer' when required.

\section{Further Application}

SPIS can be used as a last-resort safety layer for admittance controlled devices interacting with humans that would otherwise exhibit coupled instability in certain configurations or conditions.

\section{Limitations and Future Research}

The proposed method focused on simulating solely inertial virtual dynamics. Furthermore, it does not allow to perform useful work (i.e. that the device moves a human limb along a trajectory), since that would violate a strict passivity requirements. This latter problem is readily overcome by introducing an energy budget for these types of assistive movements. Future work might also look at varying the damping value more smoothly, although stability proofs of such method get more involved. Also, due to the possible absence of models of the used device, it is not always known a priori how high the switching damping value needs to be. Future work should point out if this maximum damping can be made adaptive, based on the exchanged energy of the robot back to the user. 
It is unknown how this method extends straightforwardly to flexible systems that have non-collocated motion and force measurement. However, measurements on the real haptic setup that has some lowest resonant mode below the Nyquist frequency of the controller did not show any apparent negative influence due to internal compliance.

Future work should also look into how the method can be extended to achieve not just stability, but exact marginal passivity at the human-robot interaction port. This is not trivial, since it is unknown how to divide the amount of dissipation required at the virtual model and the robot dynamics during closed-loop interaction with stiffness-like environments.

\subsection{HOW HUMANS RESPOND TO DISSIPATIVE SHARED CONTROL FORCES}

Chapter 6 presented the evaluation of a simple haptic shared controller based on damping forces around reaching targets that we call position dependent damping (PDD). Such a PDD shared controller is useful in e.g. industrial picking and placement of (heavy) objects.

\section{Scientific Contribution and Context}

We found that objects could be placed more accurately and more quickly if the reaching target area damped the motion. This is damping especially useful in tasks where lifting is assisted, where human operators tend to overshoot motions due to reduced gravity [21]. The additional benefit of using damping forces is that they can be also be generated by (almost) passive exoskeletons and lifting devices that do not require active control of forces on exoskeleton joints. This is a potential benefit of damping forces, compared to active potential forces that are used in more conventional haptic guidance.

In the experiment discussed in Chapter 6, subjects were instructed and motivated to move a mass of $12.5 \mathrm{~kg}$ as quickly as possible along a $1 \mathrm{D}$ line towards a target with a predetermined accuracy. They had to stay inside the target for $500 \mathrm{~ms}$ to be counted as a successful hit. Such an experimental design fits in the Fitts' law experimental paradigm.

In the three conditions where subjects were subjected to damping forces, they were able to reduce their movement time. Especially PDD around a reaching target reduced movement time by over $40 \%$. PDD also reduced the number of required target overshoots and reentries by a factor of 4 , increasing end-point accuracy. 
Fitts' law linear regressions between ID and movement time showed that the information-theoretical form of Fitts' law seems to hold. However, this linear fit is not the only possible fit to the data, and other non-linear curve parameterizations could have a lower residual sum of squared error than the linear regression. The only valid conclusion that can be drawn is that there was indeed an accuracy-speed trade-off in this experiment. The conditions with damping forces assisted in this trade-off for the subjects, resulting in higher possible movement speed and consequently shorter movement times.

\section{Further Application}

Using PDD could also be beneficial in rehabilitation settings or in assistive devices for daily use to increase controllability and stability. Examples of an application would be position dependent damping during table or object approaches.

\section{Limitations and Future Research}

The experiment was limited to $1 \mathrm{D}$ target approaches. To better resemble real world tasks, 3D reaching motions, possibly including peg-in-hole tasks, could show whether the positive contributions of PDD generalize to more degrees of freedom. However, in 3D a target is approachable from multiple directions. This requires the damping coefficient to be not only PDD but also direction dependent to not impede the user when a target is not necessarily approached.

Chapter 7 presented a hypothesis to explain the results observed in Chapter 6.

\section{Scientific Contribution and Context}

We hypothesize that the used constant and position dependent damping attenuated the mechanical effects of especially multiplicative noise in the humans motor control system. Higher levels of neural activation results in more absolute force noise from the muscles. Subjects were instructed to perform the reaching task as quickly as possible while conforming to the accuracy requirement. Multiplicative motor noise in their motor system would prohibit subjects to achieve higher accelerations to move faster. Because if they did, then the required endpoint accuracy could not be guaranteed. Damping forces, and especially damping around the reaching target, attenuate the mechanical effects of this multiplicative motor noise and reduce the positional variance of the end-point. Due 
to the reduction in variance, the subjects can generate more muscle force and perform the reaching movements faster than without any damping.

Stochastic non-linear optimal control models show agreement with measurements of movement kinematics and interaction forces.

\section{Further Application}

Understanding how humans interact with dynamics, for example by stochastic optimal control models, might allow designers to reverse engineer optimal interaction dynamics for shared-control-like paradigms. In this case it would allow the design of optimal damping 'profiles' to achieve a certain desired accuracy after initial learning. A practical real-world application would be the use of such a force to manually place heavy products on fittings or into machines more quickly and accurately. In such a scenario the user would be supported by a device in terms of gravity support and dissipative forces to make positioning easier, effectively reducing the effort and possibly increasing productivity and product safety.

\section{Limitations Future Research}

The experimental design used in Chapter 6 was not ideal to draw definitive conclusions to explain the observed results. Future experiments should be specifically designed to look at adaptation and investigate optimal control performed by the human subject. Furthermore, future experiments should quantify the movement variance. Results from those experiments could strengthen or falsify the current hypothesis.

For example, a future experiment where subjects tap on a surface or press a button at the target site might give a better indication of end-point variance. Performing the experiment with a single movement distance, but different target sizes might draw a clearer picture of prescribed end-point variance and the maximal accelerations humans achieve.

Furthermore, artificial multiplicative force noise could be added to the PDD force to investigate whether this henceforth increases movement time. If this does not happen, it falsifies our hypothesis and might be more indicative of benefits role-distribution found in human-human cooperation tasks. This does not explain, however, the mechanism behind why this role distribution, with two human agents both contaminated with multiplicative motor noise, would result in shorter movement time. 


\section{Optimal Control and Haptic Shared Control}

Within the H-Haptics project research was done about how humans interact with assistive haptic force fields called 'haptic guidance'. Haptic guidance experiments that show that the human is 'lazy' and exploits the haptic assistive forces [204]. We hypothesize that many of these aspects of shared control can be explained as being the result of an optimal control strategy adopted by the central nervous system (CNS). The CNS would optimize for minimal task related variance and minimal energy use (i.e. lazy behavior). However, optimal control methods cannot explain all beneficial aspects of shared control, such as cases where shared controller modulates device impedance to convey information that needs to be processed at a higher cognitive level [18].

\subsection{OVERALL CONCLUSiONS}

The work presented in this dissertation focused on improvements to haptic controller design methods and kinematic design methods that could benefit exoskeleton designers.

Rotational range of motion of the human shoulder is highly complicated and usually communicated poorly. Our new visualization method of rotational shoulder range of motion alleviates this problem by allowing to show and compare human and device range of motion, kinematic and dynamic properties and performance metrics.

To achieve a complete, body-collision-free and singularity-free rotational workspace of a robotic shoulder exoskeleton, we have presented a conceptual four degree of freedom design. The proposed inverse kinematics method allows for collision-free and singularity-free motions of the arm that would otherwise not have been possible with conventional three degree of freedom designs.

We have shown that an energy passivity requirement of admittance controlled devices leads to useful guidelines to design admittance controlled devices that can achieve large amounts of inertia reduction. Aiming for passivity is not necessarily detrimental to performance; if passivity and the preferred dynamics can be achieved while being passive and while compensating for disturbances, then both interaction stability and performance goals are achieved simultaneously. A time-domain and systems analysis of admittance control show that it is possible to remove part of the energy generate by active elements by dissipating this energy at two locations in the admittance controller; at the virtual model and at the robot mechanics through e.g. the controller. From this we arrived at a switching 
damper that makes a naive feedback-only admittance controller stable, in the sense of non-growing oscillations, when in contact with stiff environments.

We have shown that useful haptic shared control can be passive and as simple as damping around an estimated reaching target. This is an addition to the more common methods of assistive and repulsive potential forces used in haptic guidance, or mechanical constraints imposed in passive Cobot systems. Such damping forces possibly allow for haptic-guidance-like behavior on completely passive assistive devices. 

[1] Hooftman WE, Mars GMJ, Janssen B, de Vroome EMM, Janssen BJM, Michiels JEM, et al. Nationale Enquête Arbeidsomstandigheden 2015. TNO, Prevention, Work \& Health; 2016.

[2] Inspectie SZW. Gezond en veilig werken in de sector Zorg en Welzijn. Ministerie van Sociale Zaken en Werkgelegenheid; 2015.

[3] Eggink E, Oudijk D, Woittiez I. Zorgen voor Zorg, Ramingen van de vraag naar personeel in de verpleging en verzorging tot 2030. Sociaal en Cultureel Planbureau; 2010.

[4] De Looze MP, Bosch T, Krause F, Stadler KS, OSullivan LW. Exoskeletons for industrial application and their potential effects on physical work load. Ergonomics. 2016;59(5):671-681.

[5] Health Council of the Netherlands. Manual lifting at work. The Hague: Health Council of the Netherlands; 2012. Report 2012/36.

[6] Waters TR, Putz-Anderson V, Garg A, for Occupational Safety NI, Health. Applications manual for the revised NIOSH lifting equation. NIOSH; 1994.

[7] Magnusson M, Aleksiev A, Wilder D, Pope M, Spratt K, Lee S, et al. European spine societyThe acromed prize for spinal research 1995 unexpected load and asymmetric posture as etiologic factors in low back pain. European Spine Journal. 1996;5(1):23-35.

[8] Waters TR, Baron SL, Piacitelli LA, Anderson VP, Skov T, Haring-Sweeney M, et al. Evaluation of the Revised NIOSH Lifting Equation: A Cross-Sectional Epidemiologic Study. Spine. 1999;24(4):386-394.

[9] RIVM. RIVM Cost of Illness database 2013; 2013. http://www. costofillness. $\mathrm{nl}$.

[10] Hooftman WE, Mars GMJ, Janssen B, de Vroome EMM, Janssen JEM, van den Bossche SNJ. Nationale Enquête Arbeidsomstandigheden 2013. TNO, Prevention, Work \& Health; 2014.

[11] Guizzo E, Goldstein H. The rise of the body bots. IEEE spectrum. 2005;42(10):5056. 
[12] Forner-Cordero A, Pons JL, Turowska E, Schiele A, Baydal-Bertomeu J, Garrido D, et al. Kinematics and dynamics of wearable robots. Wearable robots: biomechatronic exoskeletons. 2008;p. 47-85.

[13] Aguirre-Ollinger G, Colgate JE, Peshkin MA, Goswami A. Inertia compensation control of a one-degree-of-freedom exoskeleton for lower-limb assistance: initial experiments. Neural Systems and Rehabilitation Engineering, IEEE Transactions on. 2012;20(1):68-77.

[14] Lo HS, Xie SQ. Exoskeleton robots for upper-limb rehabilitation: State of the art and future prospects. Medical engineering \& physics. 2012 April;34(3):261-268.

[15] Maciejasz P, Eschweiler J, Gerlach-Hahn K, Jansen-Troy A, Leonhardt S, et al. A survey on robotic devices for upper limb rehabilitation. Journal of neuroengineering and rehabilitation. 2014 January;11(3).

[16] H-Haptics; 2011-2017. http://www.h-haptics.nl.

[17] Abbink DA, Mulder M. Neuromuscular analysis as a guideline in designing shared control. In: Advances in haptics. InTech; 2010. .

[18] Abbink DA, Mulder M, Boer ER. Haptic shared control: smoothly shifting control authority? Cognition, Technology \& Work. 2012;14(1):19-28.

[19] Boessenkool H, Abbink DA, Heemskerk CJ, van der Helm FC, Wildenbeest JG. A task-specific analysis of the benefit of haptic shared control during telemanipulation. IEEE Transactions on Haptics. 2013;6(1):2-12.

[20] Schorsch J, Keemink A, Stienen A, Van der Helm F, Abbink D. A novel self-aligning mechanism to decouple force and torques for a planar exoskeleton joint. Mechanical sciences. 2014;5(2):29.

[21] Schorsch J, Keemink A, Stienen A, van der Helm F, Abbink D. The influence of human-robot interaction order during fast lifting tasks for different levels of weight compensation. In: Biomedical Robotics and Biomechatronics (2014 5th IEEE RAS \& EMBS International Conference on. IEEE; 2014. p. 426-431.

[22] Barnes CJ, Van Steyn SJ, Fischer RA. The effects of age, sex, and shoulder dominance on range of motion of the shoulder. Journal of Shoulder and Elbow Surgery. 2001;10(3):242-246.

[23] Hayes K, Walton JR, Szomor ZL, Murrell GA. Reliability of five methods for assessing shoulder range of motion. Australian Journal of Physiotherapy. 2001;47(4):289-294. 
[24] Namdari S, Yagnik G, Ebaugh DD, Nagda S, Ramsey ML, Williams GR, et al. Defining functional shoulder range of motion for activities of daily living. Journal of shoulder and elbow surgery. 2012;21(9):1177-1183.

[25] Wu G, Van Der Helm FC, Veeger HD, Makhsous M, Van Roy P, Anglin C, et al. ISB recommendation on definitions of joint coordinate systems of various joints for the reporting of human joint motion-Part II: shoulder, elbow, wrist and hand. Journal of biomechanics. 2005;38(5):981-992.

[26] Schiele A, van der Helm FC. Kinematic design to improve ergonomics in human machine interaction. Neural Systems and Rehabilitation Engineering, IEEE Transactions on. 2006;14(4):456-469.

[27] Letier P, Avraam M, Veillerette S, Horodinca M, De Bartolomei M, Schiele A, et al. SAM : A 7-DOF portable arm exoskeleton with local joint control. In: Intelligent Robots and Systems, 2008. IROS 2008. IEEE/RSJ International Conference on; 2008. p. 3501-3506.

[28] Stienen AH, Hekman EE, Van der Helm FC, Prange GB, Jannink MJ, Aalsma AM, et al. Dampace: dynamic force-coordination trainer for the upper extremities. In: Rehabilitation Robotics, 2007. ICORR 2007. IEEE 10th International Conference on. IEEE; 2007. p. 820-826.

[29] Perry JC, Rosen J, Burns S. Upper-limb powered exoskeleton design. Mechatronics, IEEE/ASME Transactions on. 2007;12(4):408-417.

[30] Klein J, Spencer S, Allington J, Minakata K, Wolbrecht E, Smith R, et al. Biomimetic orthosis for the neurorehabilitation of the elbow and shoulder (BONES). In: Biomedical Robotics and Biomechatronics, 2008. BioRob 2008. 2nd IEEE RAS \& EMBS International Conference on. IEEE; 2008. p. 535-541.

[31] Park HS, Ren Y, Zhang LQ. IntelliArm: an exoskeleton for diagnosis and treatment of patients with neurological impairments. In: Biomedical Robotics and Biomechatronics, 2008. BioRob 2008. 2nd IEEE RAS \& EMBS International Conference on. IEEE; 2008. p. 109-114.

[32] Nef T, Guidali M, Riener R. ARMin III-arm therapy exoskeleton with an ergonomic shoulder actuation. Applied Bionics and Biomechanics. 2009;6(2):127142.

[33] Mullaney MJ, McHugh MP, Johnson CP, Tyler TF. Reliability of shoulder range of motion comparing a goniometer to a digital level. Physiotherapy Theory and Practice. 2010;26(5):327-333. 
[34] Otten A, Voort C, Stienen A, Aarts R, van Asseldonk E, van der Kooij H. LIMPACT: A Hydraulically Powered Self-Aligning Upper Limb Exoskeleton. IEEE/ASME Transactions on Mechatronics. 2015;20(5):2285-2298.

[35] Lenarčič J, Umek A. Simple model of human arm reachable workspace. Systems, Man and Cybernetics, IEEE Transactions on. 1994;24(8):1239-1246.

[36] Klopčar N, Tomšič M, Lenarčič J. A kinematic model of the shoulder complex to evaluate the arm-reachable workspace. Journal of biomechanics. 2007;40(1):8691.

[37] Doorenbosch CAM, Harlaar J, Veeger HEJ. The globe system: An unambiguous description of shoulder positions in daily life movements. Journal of Rehabilitation Research and Development. 2003;40:147-156.

[38] Ball SJ, Brown IE, Scott SH. MEDARM: a rehabilitation robot with 5DOF at the shoulder complex. In: Advanced intelligent mechatronics, 2007 IEEE/ASME international conference on. IEEE; 2007. p. 1-6.

[39] Carignan C, Tang J, Roderick S. Development of an exoskeleton haptic interface for virtual task training. In: Intelligent Robots and Systems, 2009. IROS 2009. IEEE/RSJ International Conference on. IEEE; 2009. p. 3697-3702.

[40] Snyder JP. Map projections-A working manual. 1395. USGPO; 1987.

[41] Schiele A, Hirzinger G. A new generation of ergonomic exoskeletons-the highperformance $\mathrm{x}$-arm-2 for space robotics telepresence. In: Intelligent Robots and Systems (IROS), 2011 IEEE/RSJ International Conference on. IEEE; 2011. p. 2158 2165.

[42] Perry JC, Rosen J. Design of a 7 degree-of-freedom upper-limb powered exoskeleton. In: Biomedical Robotics and Biomechatronics, 2006. BioRob 2006. The First IEEE/RAS-EMBS International Conference on. IEEE; 2006. p. 805-810.

[43] Stienen AH, Hekman EE, Van Der Helm FC, Van Der Kooij H. Self-Aligning Exoskeleton Axes Through Decoupling of Joint Rotations and Translations. IEEE Transactions on Robotics. 2009;25(3):628-633.

[44] Stienen AHA, Keemink AQL. Visualization of shoulder range of motion for clinical diagnostics and device development. In: Rehabilitation Robotics (ICORR), 2015 IEEE International Conference on. IEEE; 2015. p. 816-821.

[45] Culmer PR, Jackson AE, Makower S, Richardson R, Cozens JA, Levesley MC, et al. A control strategy for upper limb robotic rehabilitation with a dual robot system. Mechatronics, IEEE/ASME Transactions on. 2010 July;15(4):575-585. 
[46] Miller LM, Rosen J. Comparison of multi-sensor admittance control in joint space and task space for a seven degree of freedom upper limb exoskeleton. In: Biomedical Robotics and Biomechatronics (BioRob), 2010 3rd IEEE RAS and EMBS International Conference on. IEEE; 2010. p. 70-75.

[47] Lo HS, Xie SQ. Optimization of a redundant $4 \mathrm{R}$ robot for a shoulder exoskeleton. In: Advanced Intelligent Mechatronics (AIM), 2013 IEEE/ASME International Conference on. IEEE; 2013. p. 798-803.

[48] Lo HS, Xie SQ. Optimization and analysis of a redundant $4 \mathrm{R}$ spherical wrist mechanism for a shoulder exoskeleton. Robotica. 2014 November;p. 1-21.

[49] Lo HS, Xie SQ. An upper limb exoskeleton with an optimized 4R spherical wrist mechanism for the shoulder joint. In: Advanced Intelligent Mechatronics (AIM), 2014 IEEE/ASME International Conference on. IEEE; 2014. p. 269-274.

[50] Lu J, Chen W, Tomizuka M. Kinematic Design and Analysis of a 6-DOF Upper Limb Exoskeleton Model for a Brain-Machine Interface Study. In: Mechatronic Systems. 1; 2013. p. 293-300.

[51] Carignan C, Liszka M, Roderick S. Design of an arm exoskeleton with scapula motion for shoulder rehabilitation. In: Advanced Robotics, 2005. ICAR'05. Proceedings., 12th International Conference on. IEEE; 2005. p. 524-531.

[52] Mihelj M, Nef T, Riener R. ARMin II-7 DoF rehabilitation robot: mechanics and kinematics. In: Robotics and Automation, 2007 IEEE International Conference on. IEEE; 2007. p. 4120-4125.

[53] Nef T, Riener R. Shoulder actuation mechanisms for arm rehabilitation exoskeletons. In: Biomedical Robotics and Biomechatronics, 2008. BioRob 2008. 2nd IEEE RAS \& EMBS International Conference on. IEEE; 2008. p. 862-868.

[54] Wilson JW. Analysis and mechanization of three and four gimbal systems. NASA; 1968.

[55] Wilson JW. Four-gimbal systems for simulation display. Simulation. 1969 March;12(3):115-120.

[56] Barker LK, Houck JA. Theoretical three-and four-axis gimbal robot wrists. NASA; 1986.

[57] Carter DR, Duffey P, Bachorski S, Kägi M, Havlicsek H. Weighted least-squares based control for a four axis gimbal set. In: SPIE Defense, Security, and Sensing. International Society for Optics and Photonics; 2010. p. 76630I-76630I-12. 
[58] Siciliano B, Sciavicco L, Villani L, Oriolo G. Robotics: modelling, planning and control. Springer-Verlag Limited, London, UK; 2009.

[59] Zeng G, Hemami A. An overview of robot force control. Robotica. 1997;15(5):473482.

[60] Maples J, Becker J. Experiments in force control of robotic manipulators. In: Robotics and Automation. Proceedings. 1986 IEEE International Conference on. vol. 3. IEEE; 1986. p. 695-702.

[61] Hogan N. IMPEDANCE CONTROL: AN APPROACH TO MANIPULATION: PART I - THEORY. Journal of Dynamic Systems, Measurement and Control, Transactions of the ASME. 1985;107(1):1-7.

[62] Whitney DE. Force feedback control of manipulator fine motions. Journal of Dynamic Systems, Measurement, and Control. 1977;99(2):91-97.

[63] Newman WS. Stability and performance limits of interaction controllers. Journal of dynamic systems, measurement, and control. 1992;114(4):563-570.

[64] Albu-Schäffer A, Ott C, Hirzinger G. A passivity based cartesian impedance controller for flexible joint robots-part II: Full state feedback, impedance design and experiments. In: Robotics and Automation, 2004. Proceedings. ICRA'04. 2004 IEEE International Conference on. vol. 3. IEEE; 2004. p. 2666-2672.

[65] Albu-Schäffer A, Ott C, Hirzinger G. A unified passivity-based control framework for position, torque and impedance control of flexible joint robots. The International Journal of Robotics Research. 2007;26(1):23-39.

[66] Faulring EL, Lynch KM, Colgate JE, Peshkin MA. Haptic display of constrained dynamic systems via admittance displays. Robotics, IEEE Transactions on. 2007;23(1):101-111.

[67] Lammertse P. Admittance control and impedance control-a dual. FCS Control Systems. 2004;

[68] Adams RJ, Hannaford B. Stable haptic interaction with virtual environments. Robotics and Automation, IEEE Transactions on. 1999;15(3):465-474.

[69] Van der Linde RQ, Lammertse P, Frederiksen E, Ruiter B. The HapticMaster, a new high-performance haptic interface. In: Proc. Eurohaptics; 2002. p. 1-5.

[70] Yokokohji Y, Hollis RL, Kanade T. What you can see is what you can feeldevelopment of a visual/haptic interface to virtual environment. In: Virtual Reality Annual International Symposium, 1996., Proceedings of the IEEE 1996. IEEE; 1996. p. 46-53. 
[71] Ott C, Mukherjee R, Nakamura Y. Unified impedance and admittance control. In: Robotics and Automation (ICRA), 2010 IEEE International Conference on. IEEE; 2010. p. 554-561.

[72] Newman WS, Zhang Y. Stable interaction control and coulomb friction compensation using natural admittance control. Journal of robotic systems. 1994;11(1):3-11.

[73] Surdilovic D. Contact stability issues in position based impedance control: theory and experiments. In: Robotics and Automation, 1996. Proceedings., 1996 IEEE International Conference on. vol. 2. IEEE; 1996. p. 1675-1680.

[74] Gullapalli V, Grupen RA, Barto AG. Learning reactive admittance control. In: Robotics and Automation, 1992. Proceedings., 1992 IEEE International Conference on. IEEE; 1992. p. 1475-1480.

[75] Schimmels JM, Peshkin MA. The robustness of an admittance control law designed for force guided assembly to the disturbance of contact friction. In: Robotics and Automation, 1992. Proceedings., 1992 IEEE International Conference on. IEEE; 1992. p. 2361-2366.

[76] Lawrence DA, Stoughton RM. Position-based impedance control- Achieving stability in practice. In: AIAA Guidance, Navigation and Control Conference, Monterey, CA; 1987. p. 221-226.

[77] Colbaugh R, Glass K, et al. Simulation Studies in Manipulator Impedance Control. In: American Control Conference (ACC). IEEE; 1992. p. 2941-2946.

[78] Pelletier M, Doyon M. On the implementation and performance of impedance control on position controlled robots. In: Robotics and Automation, 1994. Proceedings., 1994 IEEE International Conference on. IEEE; 1994. p. 1228-1233.

[79] Carignan CR, Smith JA. Manipulator impedance accuracy in position-based impedance control implementations. In: Robotics and Automation, 1994. Proceedings., 1994 IEEE International Conference on. IEEE; 1994. p. 1216-1221.

[80] Heinrichs B, Sepehri N, Thornton-Trump A. Position-based impedance control of an industrial hydraulic manipulator. Control Systems, IEEE. 1997;17(1):46-52.

[81] Ott C, Nakamura Y. Base force/torque sensing for position based Cartesian impedance control. In: Intelligent Robots and Systems, 2009. IROS 2009. IEEE/RSJ International Conference on. IEEE; 2009. p. 3244-3250.

[82] Duchaine V, Gosselin CM. General model of human-robot cooperation using a novel velocity based variable impedance control. In: EuroHaptics Conference, 2007 and Symposium on Haptic Interfaces for Virtual Environment and Teleoperator Systems. World Haptics 2007. Second Joint. IEEE; 2007. p. 446-451. 
[83] Rahman M, Ikeura R, Mizutani K. Investigating the impedance characteristic of human arm for development of robots to co-operate with human operators. In: Systems, Man, and Cybernetics, 1999. IEEE SMC'99 Conference Proceedings. 1999 IEEE International Conference on. vol. 2. IEEE; 1999. p. 676-681.

[84] Aguirre-Ollinger G, Colgate JE, Peshkin MA, Goswami A. Active-impedance control of a lower-limb assistive exoskeleton. In: Rehabilitation Robotics, 2007. ICORR 2007. IEEE 10th International Conference on. IEEE; 2007. p. 188-195.

[85] Seraji H, Colbaugh R. Force tracking in impedance control. The International Journal of Robotics Research. 1997;16(1):97-117.

[86] Ueberle M, Buss M. Control of kinesthetic haptic interfaces. In: Proc. IEEE/RSJ Int. Conf. on Intellig. Rob. and Syst., Workshop on Touch and Haptics. vol. 22; 2004. .

[87] Carignan CR, Cleary KR. Closed-loop force control for haptic simulation of virtual environments. Haptics-e. 2000;1(2):1-14.

[88] Dohring M, Newman W. The passivity of natural admittance control implementations. In: Robotics and Automation, 2003. Proceedings. ICRA'03. IEEE International Conference on. vol. 3. IEEE; 2003. p. 3710-3715.

[89] Colgate JE, Hogan N. Robust control of dynamically interacting systems. International journal of Control. 1988;48(1):65-88.

[90] Nambi M, Provancher WR, Abbott JJ. On the ability of humans to apply controlled forces to admittance-type devices. Advanced Robotics. 2011;25(5):629-650.

[91] Carmichael MG, Liu D. Admittance control scheme for implementing modelbased assistance-as-needed on a robot. In: Engineering in Medicine and Biology Society (EMBC), 2013 35th Annual International Conference of the IEEE. IEEE; 2013. p. $870-873$.

[92] Colgate JE. The control of dynamically interacting systems. Massachusetts Institute of Technology; 1988.

[93] Schimmels JM, Peshkin MA. Force-assembly with friction. Robotics and Automation, IEEE Transactions on. 1994;10(4):465-479.

[94] Glosser GD, Newman WS. The implementation of a natural admittance controller on an industrial manipulator. In: Robotics and Automation, 1994. Proceedings., 1994 IEEE International Conference on. IEEE; 1994. p. 1209-1215.

[95] Bascetta L, Ferretti G, Magnani G, Rocco P. Walk-through programming for robotic manipulators based on admittance control. Robotica. 2013;31(07):1143-1153. 
[96] Clover C. A control-system architecture for robots used to simulate dynamic force and moment interaction between humans and virtual objects. Systems, Man, and Cybernetics, Part C: Applications and Reviews, IEEE Transactions on. 1999;29(4):481-493.

[97] Adams RJ, Hannaford B. Control law design for haptic interfaces to virtual reality. Control Systems Technology, IEEE Transactions on. 2002;10(1):3-13.

[98] Strolz M, Buss M. Haptic rendering of actuated mechanisms by active admittance control. In: Haptics: Perception, Devices and Scenarios. Springer; 2008. p. 712717.

[99] Hogan N. Controlling impedance at the man/machine interface. In: Robotics and Automation, 1989. Proceedings., 1989 IEEE International Conference on. IEEE; 1989. p. 1626-1631.

[100] Kazerooni H. Human-robot interaction via the transfer of power and information signals. Systems, Man and Cybernetics, IEEE Transactions on. 1990;20(2):450-463.

[101] Van der Linde R, Lammertse P. HapticMaster-a generic force controlled robot for human interaction. Industrial Robot: An International Journal. 2003;30(6):515524.

[102] Meuleman J, van Asseldonk E, van der Kooij H. Novel actuation design of a gait trainer with shadow leg approach. In: IEEE... International Conference on Rehabilitation Robotics:[proceedings]. vol. 2013; 2013. p. 1-8.

[103] Moog Inc. High Fidelity Simulation; 2014. Available from: ht tp : / /www . moog. com.

[104] Faulring EL, Colgate JE, Peshkin MA. A high performance 6-DOF haptic cobot. In: Robotics and Automation, 2004. Proceedings. ICRA'04. 2004 IEEE International Conference on. vol. 2. IEEE; 2004. p. 1980-1985.

[105] Kragic D, Marayong P, Li M, Okamura AM, Hager GD. Human-machine collaborative systems for microsurgical applications. The International Journal of Robotics Research. 2005;24(9):731-741.

[106] Lee J, Kim K, Chung WK, Choi S, Kim YS. Human-guided surgical robot system for spinal fusion surgery: CoRASS. In: Robotics and Automation, 2008. ICRA 2008. IEEE International Conference on. IEEE; 2008. p. 3881-3887.

[107] Miller LM, Rosen J. Comparison of multi-sensor admittance control in joint space and task space for a seven degree of freedom upper limb exoskeleton. In: Biomedical Robotics and Biomechatronics (BioRob), 2010 3rd IEEE RAS and EMBS International Conference on. IEEE; 2010. p. 70-75. 
[108] Yu W, Rosen J, Li X. PID admittance control for an upper limb exoskeleton. In: American Control Conference (ACC), 2011. IEEE; 2011. p. 1124-1129.

[109] Huo W, Huang J, Wang Y, Wu J, Cheng L. Control of upper-limb power-assist exoskeleton based on motion intention recognition. In: Robotics and Automation (ICRA), 2011 IEEE International Conference on. IEEE; 2011. p. 2243-2248.

[110] Kim H, Miller LM, Li Z, Roldan JR, Rosen J. Admittance control of an upper limb exoskeleton-Reduction of energy exchange. In: Engineering in Medicine and Biology Society (EMBC), 2012 Annual International Conference of the IEEE. IEEE; 2012. p. 6467-6470.

[111] Colombo R, Pisano F, Micera S, Mazzone A, Delconte C, Carrozza MC, et al. Robotic techniques for upper limb evaluation and rehabilitation of stroke patients. Neural Systems and Rehabilitation Engineering, IEEE Transactions on. 2005;13(3):311-324.

[112] Culmer P, Jackson A, Levesley M, Savage J, Richardson R, Cozens J, et al. An admittance control scheme for a robotic upper-limb stroke rehabilitation system. In: Proceedings of the 2005 IEEE Engineering in Medicine and Biology 27th Annual Conference; 2005. .

[113] Stienen AH, Hekman EE, ter Braak H, Aalsma AM, van der Helm FC, van der Kooij H. Design of a rotational hydroelastic actuator for a powered exoskeleton for upper limb rehabilitation. Biomedical Engineering, IEEE Transactions on. 2010;57(3):728-735.

[114] Ozkul F, Erol Barkana D. Design of an admittance control with inner robust position control for a robot-assisted rehabilitation system RehabRoby. In: Advanced Intelligent Mechatronics (AIM), 2011 IEEE/ASME International Conference on. IEEE; 2011. p. 104-109.

[115] Stienen AH, McPherson JG, Schouten AC, Dewald J. The ACT-4D: a novel rehabilitation robot for the quantification of upper limb motor impairments following brain injury. In: Rehabilitation Robotics (ICORR), 2011 IEEE International Conference on. IEEE; 2011. p. 1-6.

[116] Bortole M, del Ama A, Rocon E, Moreno J, Brunetti F, Pons J. A robotic exoskeleton for overground gait rehabilitation. In: Robotics and Automation (ICRA), 2013 IEEE International Conference on. IEEE; 2013. p. 3356-3361.

[117] Patton J, Brown DA, Peshkin M, Santos-Munné JJ, Makhlin A, Lewis E, et al. KineAssist: design and development of a robotic overground gait and balance therapy device. Topics in stroke rehabilitation. 2008;15(2):131-139. 
[118] Wang D, Li J, Li C. An adaptive haptic interaction architecture for knee rehabilitation robot. In: Mechatronics and Automation, 2009. ICMA 2009. International Conference on. IEEE; 2009. p. 84-89.

[119] Aguirre-Ollinger G, Colgate JE, Peshkin MA, Goswami A. Design of an active one-degree-of-freedom lower-limb exoskeleton with inertia compensation. The International Journal of Robotics Research. 2011;30(4):486-499.

[120] Saglia JA, Tsagarakis NG, Dai JS, Caldwell DG. Control strategies for ankle rehabilitation using a high performance ankle exerciser. In: Robotics and Automation (ICRA), 2010 IEEE International Conference on. IEEE; 2010. p. 2221-2227.

[121] Kazerooni H, Guo J. Human extenders. Journal of dynamic systems, measurement, and control. 1993;115(2B):281-290.

[122] Colgate JE, Peshkin MA, Klostermeyer SH. Intelligent assist devices in industrial applications: a review. In: IROS; 2003. p. 2516-2521.

[123] Surdilovic D, Radojicic J. Robust control of interaction with haptic interfaces. In: Robotics and Automation, 2007 IEEE International Conference on. IEEE; 2007. p. 3237-3244.

[124] Lecours A, Mayer-St-Onge B, Gosselin C. Variable admittance control of a fourdegree-of-freedom intelligent assist device. In: Robotics and Automation (ICRA), 2012 IEEE International Conference on. IEEE; 2012. p. 3903-3908.

[125] Li Z, Tsagarakis NG, Caldwell DG. A passivity based admittance control for stabilizing the compliant humanoid COMAN. In: Humanoid Robots (Humanoids), 2012 12th IEEE-RAS International Conference on. IEEE; 2012. p. 43-49.

[126] Okunev V, Nierhoff T, Hirche S. Human-preference-based control design: Adaptive robot admittance control for physical human-robot interaction. In: RO-MAN, 2012 IEEE. IEEE; 2012. p. 443-448.

[127] Yamada D, Huang J, Yabuta T. Comparison Between Admittance and Impedance Control of a Multi-Finger-Arm Robot using the Guaranteed Manipulability Method. Precision Instrument and Mechanology. 2013;2(1):85-93.

[128] Augugliaro F, D’Andrea R. Admittance control for physical human-quadrocopter interaction. In: Control Conference (ECC), 2013 European. IEEE; 2013. p. 18051810.

[129] Wang H, Patota F, Buondonno G, Haendl M, De Luca A, Kosuge K. Stability and variable admittance control in the physical interaction with a mobile robot. International Journal of Advanced Robotic Systems. 2015;12(12):173. 
[130] Prabhu SM, Garg DP. Fuzzy-logic-based reinforcement learning of admittance control for automated robotic manufacturing. Engineering Applications of Artificial Intelligence. 1998;11(1):7-23.

[131] Dimeas F, Koustoumpardis P, Aspragathos N. Admittance neuro-control of a lifting device to reduce human effort. Advanced Robotics. 2013;27(13):1013-1022.

[132] Yu W, Rodriguez RC, Li X. Neural PID admittance control of a robot. In: American Control Conference (ACC), 2013. IEEE; 2013. p. 4963-4968.

[133] Lawrence DA. Stability and transparency in bilateral teleoperation. Robotics and Automation, IEEE Transactions on. 1993;9(5):624-637.

[134] Hashtrudi-Zaad K, Salcudean SE. Analysis of control architectures for teleoperation systems with impedance/admittance master and slave manipulators. The International Journal of Robotics Research. 2001;20(6):419-445.

[135] Colgate JE, Brown JM. Factors affecting the z-width of a haptic display. In: Robotics and Automation, 1994. Proceedings., 1994 IEEE International Conference on. IEEE; 1994. p. 3205-3210.

[136] Liberzon D. Switching in systems and control. Springer; 2003.

[137] Raisbeck G. A definition of passive linear networks in terms of time and energy. Journal of Applied Physics. 1954;25(12):1510-1514.

[138] Buerger SP, Hogan N. Relaxing passivity for human-robot interaction. In: Intelligent Robots and Systems, 2006 IEEE/RSJ International Conference on. IEEE; 2006. p. $4570-4575$.

[139] Willaert B, Corteville B, Reynaerts D, Van Brussel H, Vander Poorten EB. Bounded environment passivity of the classical position-force teleoperation controller. In: Intelligent Robots and Systems, 2009. IROS 2009. IEEE/RSJ International Conference on. IEEE; 2009. p. 4622-4628.

[140] Haddadi A. Stability, Performance, and Implementation Issues in Bilateral Teleoperation Control and Haptic Simulation Systems. Queen's University at Kingston, Canada; 2011.

[141] Dyck M, Jazayeri A, Tavakoli M. Is the human operator in a teleoperation system passive? In: World Haptics Conference (WHC), 2013. IEEE; 2013. p. 683-688.

[142] Aung MTS, Kikuuwe R. Acceleration feedback and friction compensation for improving the stability of admittance control. In: Control Conference (ASCC), 2015 10th Asian. IEEE; 2015. p. 1-6. 
[143] Colgate E, Hogan N. An analysis of contact instability in terms of passive physical equivalents. In: Robotics and Automation, 1989. Proceedings., 1989 IEEE International Conference on. IEEE; 1989. p. 404-409.

[144] Keemink AQL, van der Kooij H, Stienen AHA. Admittance Control for Physical Human Machine Interaction [under Review]. International Journal of Robotics Research. 2017;

[145] De Stefano M, Artigas J, Rackl W, Albu-Schaeffer A. Passivity of virtual freefloating dynamics rendered on robotic facilities. In: Robotics and Automation (ICRA), 2015 IEEE International Conference on. IEEE; 2015. p. 781-788.

[146] Nabeel M, Lee J, Mehmood U, Jafari A, Hwang JH, Ryu JH. Increasing the impedance range of admittance-type haptic interfaces by using Time Domain Passivity Approach. In: Intelligent Robots and Systems (IROS), 2015 IEEE/RSJ International Conference on. IEEE; 2015. p. 585-590.

[147] Hannaford B, Ryu JH. Time-domain passivity control of haptic interfaces. IEEE Transactions on Robotics and Automation. 2002;18(1):1-10.

[148] Ryu JH, Hannaford B, Preusche C, Hirzinger G. Time domain passivity control with reference energy behavior. In: Intelligent Robots and Systems, 2003.(IROS 2003). Proceedings. 2003 IEEE/RSJ International Conference on. vol. 3. IEEE; 2003. p. 2932-2937.

[149] Stramigioli S, Secchi C, van der Schaft AJ, Fantuzzi C. A novel theory for sampled data system passivity. In: Intelligent Robots and Systems, 2002. IEEE/RSJ International Conference on. vol. 2. IEEE; 2002. p. 1936-1941.

[150] Totorkulov K, Ryu JH. Stable haptic interaction with admittance type virtual environments based on time-domain passivity approach. In: Ubiquitous Robots and Ambient Intelligence (URAI), 2012 9th International Conference on. IEEE; 2012. p. 111-113.

[151] Paynter HM. Analysis and design of engineering systems. MIT press; 1961.

[152] Kim MJ, Lee W, Ott C, Chung WK. A passivity-based admittance control design using feedback interconnections. In: Intelligent Robots and Systems (IROS), 2016 IEEE/RSJ International Conference on. IEEE; 2016. p. 801-807.

[153] Rebelo J, Schiele A. Time domain passivity controller for 4-channel time-delay bilateral teleoperation. IEEE transactions on haptics. 2015;8(1):79-89.

[154] Morbi A, Ahmadi M. Safely rendering small impedances in admittance-controlled haptic devices. IEEE/ASME Transactions on Mechatronics. 2016;21(3):1272-1280. 
[155] van Oosterhout J, Wildenbeest JG, Boessenkool H, Heemskerk CJ, de Baar MR, van der Helm FC, et al. Haptic Shared Control in Tele-Manipulation: Effects of Inaccuracies in Guidance on Task Execution. Haptics, IEEE Transactions on. 2015;8(2):164-175.

[156] Lecours A, Mayer-St-Onge B, Gosselin C. Variable admittance control of a fourdegree-of-freedom intelligent assist device. In: Robotics and Automation (ICRA), 2012 IEEE International Conference on. IEEE; 2012. p. 3903-3908.

[157] Lockheed Martin. FORTIS Exoskeleton; 2016. [Online; accessed 1-March-2016]. http://www. lockheedmartin.com/us/products/exoskeleton/FORTIS.html.

[158] Collins SH, Wiggin MB, Sawicki GS. Reducing the energy cost of human walking using an unpowered exoskeleton. Nature. 2015;522(7555):212-215.

[159] Fitts PM. The information capacity of the human motor system in controlling the amplitude of movement. Journal of experimental psychology. 1954;47(6):381.

[160] Fitts PM, Peterson JR. Information capacity of discrete motor responses. Journal of experimental psychology. 1964;67(2):103.

[161] Bernier PM, Chua R, Bard C, Franks IM. Updating of an internal model without proprioception: a deafferentation study. Neuroreport. 2006;17(13):1421-1425.

[162] Burdet E, Franklin DW, Milner TE. Human Robotics: Neuromechanics and Motor Control. MIT Press; 2013.

[163] Soukoreff RW, MacKenzie IS. Towards a standard for pointing device evaluation, perspectives on 27 years of Fitts' law research in HCI. International journal of human-computer studies. 2004;61(6):751-789.

[164] Kerr R. Movement time in an underwater environment. Journal of Motor Behavior. 1973;5(3):175-178.

[165] Kerr R. Diving, adaptation, and Fitts law. Journal of Motor Behavior. 1978;10(4):255-260.

[166] Richard C, Cutkosky M. The effects of real and computer generated friction on human performance in a targeting task. In: Proceedings of the ASME Dynamic Systems and Control Division. vol. 69; 2000. p. 2.

[167] Berkelman P, Ma J. Effects of friction parameters on completion times for sustained planar positioning tasks with a haptic interface. In: Intelligent Robots and Systems, 2006 IEEE/RSJ International Conference on. IEEE; 2006. p. 1115-1120. 
[168] Crommentuijn K, Hermes DJ. The effect of coulomb friction in a haptic interface on positioning performance. In: Haptics: Generating and Perceiving Tangible Sensations. Springer; 2010. p. 398-405.

[169] Chapuis O, Dragicevic P. Small targets: why are they so difficult to acquire. Laboratoire de Recherche en Informatique; 2008.

[170] Chapuis O, Dragicevic P. Effects of motor scale, visual scale, and quantization on small target acquisition difficulty. ACM Transactions on Computer-Human Interaction (TOCHI). 2011;18(3):13.

[171] Van der Linde RQ, Lammertse P, Frederiksen E, Ruiter B. The HapticMaster, a new high-performance haptic interface. In: Proc. Eurohaptics; 2002. p. 1-5.

[172] Sainburg R, Kalakanis D. Differences in control of limb dynamics during dominant and nondominant arm reaching. Journal of neurophysiology. 2000;83(5):26612675.

[173] Glazebrook CM, Kiernan D, Welsh TN, Tremblay L. How one breaks Fitts's Law and gets away with it: Moving further and faster involves more efficient online control. Human movement science. 2015;39:163-176.

[174] Ware C, Mikaelian HH. An evaluation of an eye tracker as a device for computer input. In: ACM SIGCHI Bulletin. vol. 17. ACM; 1987. p. 183-188.

[175] Hansen JP, Johansen AS, Hansen DW, Itoh K, Mashino S. Command without a click: Dwell time typing by mouse and gaze selections. In: Proceedings of HumanComputer Interaction-INTERACT; 2003. p. 121-128.

[176] Miyamoto H, Nakano E, Wolpert DM, Kawato M. TOPS (Task Optimization in the Presence of Signal-Dependent Noise) model. Systems and Computers in Japan. 2004;35(11):48-58.

[177] Welford A, Norris A, Shock N. Speed and accuracy of movement and their changes with age. Acta psychologica. 1969;30:3-15.

[178] Rosenberg LB. The Use of Virtual Fixtures as Perceptual Overlays to Enhance Operator Performance in Remote Environments. DTIC Document; 1992.

[179] Keemink AQL, Fierkens RIK, Lobo-Prat J, Schorsch JSF, Abbink DA, Smeets JBJ, et al. Using position dependent damping forces around reaching targets for transporting heavy objects: A Fitts' law approach. In: Biomedical Robotics and Biomechatronics (BioRob), 2016 6th IEEE International Conference on. IEEE; 2016. p. $1323-1329$. 
[180] Guiard Y, Beaudouin-Lafon M. Fitts' law 50 years later: applications and contributions from human-computer interaction. Academic Press; 2004.

[181] Goodbody SJ, Wolpert DM. Temporal and amplitude generalization in motor learning. Journal of Neurophysiology. 1998;79(4):1825-1838.

[182] Conditt MA, Mussa-Ivaldi FA. Central representation of time during motor learning. Proceedings of the National Academy of Sciences. 1999;96(20):11625-11630.

[183] Shadmehr R, Mussa-Ivaldi FA. Adaptive representation of dynamics during learning of a motor task. Journal of Neuroscience. 1994;14(5):3208-3224.

[184] DiZio P, Lackner JR. Congenitally blind individuals rapidly adapt to Coriolis force perturbations of their reaching movements. Journal of Neurophysiology. 2000;84(4):2175-2180.

[185] Karniel A, Mussa-Ivaldi FA. Sequence, time, or state representation: how does the motor control system adapt to variable environments? Biological cybernetics. 2003;89(1):10-21.

[186] Shadmehr R, Wise SP. The computational neurobiology of reaching and pointing: a foundation for motor learning. MIT press; 2005.

[187] Miall RC, Wolpert DM. Forward models for physiological motor control. Neural networks. 1996;9(8):1265-1279.

[188] Kawato M. Internal models for motor control and trajectory planning. Current opinion in neurobiology. 1999;9(6):718-727.

[189] Flash T, Hogan N. The coordination of arm movements: an experimentally confirmed mathematical model. Journal of neuroscience. 1985;5(7):1688-1703.

[190] Harris CM, Wolpert DM. Signal-dependent noise determines motor planning. Nature. 1998;394(6695):780-784.

[191] Izawa J, Rane T, Donchin O, Shadmehr R. Motor adaptation as a process of reoptimization. Journal of Neuroscience. 2008;28(11):2883-2891.

[192] Jones KE, Hamilton AFdC, Wolpert DM. Sources of signal-dependent noise during isometric force production. Journal of neurophysiology. 2002;88(3):1533-1544.

[193] Todorov E, Jordan MI. Optimal feedback control as a theory of motor coordination. Nature neuroscience. 2002;5(11):1226-1235.

[194] Todorov E. Stochastic optimal control and estimation methods adapted to the noise characteristics of the sensorimotor system. Neural computation. 2005;17(5):1084-1108. 
[195] Shadmehr R, Krakauer JW. A computational neuroanatomy for motor control. Experimental Brain Research. 2008;185(3):359-381.

[196] Izawa J, Shadmehr R. On-line processing of uncertain information in visuomotor control. Journal of Neuroscience. 2008;28(44):11360-11368.

[197] Diedrichsen J, Shadmehr R, Ivry RB. The coordination of movement: optimal feedback control and beyond. Trends in cognitive sciences. 2010;14(1):31-39.

[198] Diedrichsen J. Optimal task-dependent changes of bimanual feedback control and adaptation. Current Biology. 2007;17(19):1675-1679.

[199] Todorov E, Li W. A generalized iterative LQG method for locally-optimal feedback control of constrained nonlinear stochastic systems. In: American Control Conference, 2005. Proceedings of the 2005. IEEE; 2005. p. 300-306.

[200] Li W, Todorov E. Iterative linearization methods for approximately optimal control and estimation of non-linear stochastic system. International Journal of Control. 2007;80(9):1439-1453.

[201] Reed KB, Peshkin MA. Physical Collaboration of Human-Human and HumanRobot teams. IEEE Transactions on Haptics. 2008;1(2):108-120.

[202] Mazzoni P, Hristova A, Krakauer JW. Why don't we move faster? Parkinson's disease, movement vigor, and implicit motivation. Journal of neuroscience. 2007;27(27):7105-7116.

[203] Nesselaar AW, Keemink AQL, Stienen AHA. Analyse van de meetmethode voor het bewegingsbereik van de schouder met de Mollweide projectie visualisatie. University of Twente, Biomechanical Enginereering; 2015. Report BW-476.

[204] de Jonge AW, Wildenbeest JG, Boessenkool H, Abbink DA. The effect of Trialby-Trial adaptation on conflicts in haptic shared control for free-air teleoperation tasks. IEEE transactions on haptics. 2016;9(1):111-120. 

Part V

A P P E NDIX 

To preserve scaling in plotting applications, the extremes of the Mollweide projection (the poles and the lateral 'sides') are mapped onto to the range: $y \in$ $\left[0^{\circ}, 180^{\circ}\right], x \in\left[-90^{\circ}, 270^{\circ}\right)$. These four extremes are then mapped to the same points as for an equirectangular projection. This scaling is preserved if the following is used to calculate the projected spherical coordinates $\gamma_{h}$ and $\gamma_{e}$ (given in degrees) onto a $2 \mathrm{D}$ plane:

$$
\begin{aligned}
& x=R \frac{2 \sqrt{2}}{\pi} \cos (\theta) \frac{\left(\gamma_{h}-90^{\circ}\right) \pi}{180^{\circ}}+90^{\circ} \\
& y=R \sqrt{2} \sin (\theta)+90^{\circ}, R=\frac{90^{\circ}}{\sqrt{2}}
\end{aligned}
$$

with the implicit equation

$$
\theta=\frac{\pi \sin \left(\gamma_{e}-90^{\circ}\right)-\sin (2 \theta)}{2} .
$$

Note in (A.1) that $\gamma_{e}$ is entered in degrees $\left(\left[^{\circ}\right]\right)$. Care has to be taken in software applications by possibly converting the value to radians for a proper $\sin (\cdot)$ argument.

Eq. (A.1) can be solved by Newton-Raphson iteration [40]:

$$
\theta_{n+1}=\theta_{n}-\frac{2 \theta_{n}+\sin \left(2 \theta_{n}\right)-\pi \sin \left(\gamma_{e}-90^{\circ}\right)}{2+2 \cos \left(2 \theta_{n}\right)},
$$

which has to be repeated until convergence and will give a value in radians. 

An Euler-equation of rotational motion (or something similar) can be described in the rotating upper-arm frame, describing the angular acceleration $\left({ }^{u a} \dot{\boldsymbol{\Omega}}\right)$ of a rotating rigid body. The acceleration is integrated to rotational velocity shown in Fig. 3.2a. The rotational acceleration and velocities have to be transformed between the global and the moving frame:

$$
\begin{aligned}
{ }^{g} \boldsymbol{\Omega} & ={ }^{g} \mathbf{R}_{u a}{ }^{u a} \boldsymbol{\Omega} \\
{ }^{g} \dot{\boldsymbol{\Omega}} & ={ }^{g} \mathbf{R}_{u a}{ }^{u a} \dot{\mathbf{\Omega}}+{ }^{g} \dot{\mathbf{R}}_{u a}{ }^{u a} \boldsymbol{\Omega} \\
& ={ }^{g} \mathbf{R}_{u a}{ }^{u a} \dot{\mathbf{\Omega}}+{ }^{g} \boldsymbol{\Omega} \times{ }^{g} \mathbf{R}_{u a}{ }^{u a} \boldsymbol{\Omega} .
\end{aligned}
$$

\section{BODY COLLISIONS}

The actual constraints in jointspace are determined through the forward kinematics, moving a parameterized circle (of all motors their front face, which is closest to the exoskeleton CoR) in 3D space:

$$
{ }^{g} \mathbf{c}_{j}(\mathbf{q})={ }^{g} \mathbf{R}_{j}(\mathbf{q})^{j} \mathbf{c}_{j}={ }^{g} \mathbf{R}_{j}(\mathbf{q})\left[\begin{array}{c}
r_{m o} \sin (\zeta) \\
r_{j} \\
r_{m o} \cos (\zeta)
\end{array}\right], j=2,3,
$$

with $\zeta \in[0,2 \pi]$ to make a full circle, $r_{j}$ the distance of the $j$-th motor's front face to the center of rotation and $r_{m o}$ the radius of the motor's front face. To be a valid configuration, the following condition are valid if it holds for all $\zeta \in[0,2 \pi]$ :

$$
{ }^{g} c_{j, z} \geq 0 \vee{ }^{g} c_{j, x} \leq-d_{t r} \vee\left({ }^{g} c_{j, z} \geq d_{t r} \wedge{ }^{g} c_{j, x} \leq 0 \wedge\left\|\left[\begin{array}{c}
g^{g} c_{j, x} \\
g_{c_{j, z}-d_{t r}}
\end{array}\right]\right\|_{2} \geq d_{t r}\right) .
$$


The first condition states that the motor is on the 'right' (positive in $\mathbf{z}_{g}$ ) of the global $X Y$-plane. The second condition states that the motor is behind (negative in $\mathbf{x}_{g}$ ) the $Y Z$-plane behind the back. The third condition states that the motor is outside the quarter cylinder connecting both planes.

\section{ARM COLLISIONS}

The constraint can be denoted as follows:

$$
{ }^{g} \mathbf{u}_{u a} \cdot{ }^{g} \omega_{j} \leq \cos \left(\alpha_{u a, j}\right), j=1,2,3
$$

The angles $\alpha_{u a, j}$ are found through the implicit equation:

$$
r_{j} \sin \left(\alpha_{u a, j}\right)=r_{m o} \cos \left(\alpha_{u a, j}\right)+r_{u a}
$$

which can be solved through an imaginary detour, swapping the trigonometric functions with their complex exponents, yielding:

$$
\alpha_{u a, j}=-i \ln \left(\frac{\sqrt{r_{m o}^{2}+r_{j}^{2}-r_{u a}^{2}}+i r_{u a}}{r_{j}-i r_{m o}}\right), i=\sqrt{-1} \text {, }
$$

with $\ln (\cdot)$ the natural logarithm. This equation yields a real solution for the collision cone angle.

First, we check if the upper arm is between two motor axes. Where $\mathbf{p}_{j}$ is the normalized projection vector of $\mathbf{u}_{u a}$ on the plane generated by ${ }^{g} \omega_{j}$ and ${ }^{g} \omega_{j+1}$.

$$
\begin{aligned}
{ }^{g} \mathbf{n}_{j} & =\frac{{ }^{g} \boldsymbol{\omega}_{j} \times{ }^{g} \boldsymbol{\omega}_{j+1}}{\left\|\boldsymbol{\omega}_{j} \times{ }^{g} \boldsymbol{\omega}_{j+1}\right\|} \\
{ }^{g} \mathbf{p}_{j} & =\frac{{ }^{g} \mathbf{n}_{j} \times\left({ }^{g} \mathbf{u}_{u a} \times{ }^{g} \mathbf{n}_{j}\right)}{\left\|\mathbf{n}_{j} \times\left({ }^{g} \mathbf{u}_{u a} \times{ }^{g} \mathbf{n}_{j}\right)\right\| .}
\end{aligned}
$$

The sum of the two absolute angles between $\mathbf{p}_{j}$ and the rotation axes has to be more than the angle between both rotation axes (the $\operatorname{arc} \phi_{j}$ ):

$$
\arccos \left({ }^{g} \mathbf{p}_{j} \cdot{ }^{g} \boldsymbol{\omega}_{j}\right)+\arccos \left({ }^{g} \mathbf{p}_{j} \cdot{ }^{g} \boldsymbol{\omega}_{j+1}\right)>\phi_{j}, j=1,2
$$


If the above constraint does not hold, this is only problematic if the arm is close enough to the plane formed by the rotation axes to hit the link. Therefore it should hold that:

$$
\left|{ }^{g} \mathbf{n}_{j} \cdot{ }^{g} \mathbf{u}_{u a}\right| \leq \sin \left(\alpha_{l i, j}\right), j=1,2
$$

where $\alpha_{l i, j}$ is found in a similar way as in (B.1), but by substituting $r_{m o}$ with $d_{l i} / 2$. Both motor and link collision conditions are kinematically only dependent on $\phi_{1}, \phi_{2}, q_{2}$ and $q_{3}$.

\section{CONDITIONING}

The sub-determinants of Jacobian $\mathbf{J}(\mathbf{q})$ are given by:

$$
\begin{aligned}
& \Delta_{1}=\operatorname{det}\left[{ }^{g} \omega_{1}{ }^{g} \omega_{2}{ }^{g} \omega_{3}\right] \\
& \Delta_{2}=\operatorname{det}\left[{ }^{g} \omega_{2}{ }^{g} \omega_{3}{ }^{g} \omega_{4}\right] \\
& \Delta_{3}=\operatorname{det}\left[{ }^{g} \omega_{1}{ }^{g} \omega_{2}{ }^{g} \omega_{4}\right] \\
& \Delta_{4}=\operatorname{det}\left[{ }^{g} \omega_{1}{ }^{g} \omega_{3}{ }^{g} \omega_{4}\right] .
\end{aligned}
$$

The condition for proper conditioning is then:

$$
\max \left(\left[\left|\Delta_{1}\right|,\left|\Delta_{2}\right|,\left|\Delta_{3}\right|,\left|\Delta_{4}\right|\right]\right) \geq \eta
$$

with $\eta \approx \frac{\|\Omega\|_{\max }}{\left\|\dot{\mathbf{q}}_{\max }\right\|}$, approximately the ratio between the shoulder and exoskeleton velocities for minimum norm solutions.

\section{ALGORITHM LIMIT CASES}

If $T \rightarrow 0$ this algorithm reduces to a continuous time version, which removes the distinction between $\mathbf{q}_{k-1}$ and $\mathbf{q}_{k}^{+}$. If the gain $K$ is high, the saturation will clip the highest velocity to some predefined bound. This way, the convergence rate can be increased up to when the exoskeleton moving as fast as possible along $\mathcal{Q}_{n s}\left(\mathbf{q}_{k}^{+}\right)$. This is a valid, but more aggressive solution to the problem, giving high joint speeds and accelerations. 
BOUNDS ON $K$

We recognize (3.25) to be a discrete Euler integrator with a gain $K$ in a closed loop. This process has a pole location at $p=1-K T$ in the $z$-domain. For stable convergence $|p|<1$. This gives as a gain condition: $0 \leq K<2 / T$. To have nonoscillating exponential convergence the upper bound on $K$ is actually $1 / T$.

\section{FINDING THE NULLSPACE-CONFIGURATION SET EFFICIENTLY}

In computer applications it is preferred to numerically integrate (3.15) in two directions from $\mathbf{q}$ up to some point that the end-points (modulo $2 \pi$ ) meet (the difference is smaller than 2 integration steps). Since the eigenvector $\mathbf{u}_{\mathcal{N}}$ acquired through numerical algorithms can have 'random' sign, every discrete spatial integration instant $\kappa$ (not to be confused with temporal integration of the motion over time instant $k$ ) a direction correction is made through the inner product of $\mathbf{u}_{\mathcal{N}}$ of the current $(\kappa)$ and its values of the previous integration step $(\kappa-1)$ :

$$
\mathbf{u}_{\mathcal{N}, \kappa, \text { corr }}=\mathbf{u}_{\mathcal{N}, \kappa} \operatorname{sgn}\left(\mathbf{u}_{\mathcal{N}, \kappa} \cdot \mathbf{u}_{\mathcal{N}, \kappa-1}\right)
$$

FINDING $\xi^{0}$

Eq. (3.22) is in computer applications calculated by finding a the minimal value of $\delta(\xi)$ in the array of all elements of the nullspace-configuration curve. Since the spatial integration in (3.15) goes with unit-velocity, the array-index $i_{\text {min }}$ belonging to $\mathbf{q}_{k}^{m}$ is directly proportional to $\xi$ with integration step $\Delta x$. Therefore $\xi^{m} \rho=i_{\text {min }} \Delta x \rho$. 
The full transfer function for the system shown in Figure 4.4 is given by

$$
\begin{aligned}
Y_{a} & =\frac{v}{F_{\text {ext }}} \\
& =\frac{Y_{r}\left(H_{d} S_{f}\left(G_{f}+C^{\prime} Y_{v}^{*}\right)+1\right)}{Y_{r}\left(Z_{p s}+D\right)+1},
\end{aligned}
$$

with

$$
\begin{aligned}
D & =H_{d}\left(C_{f b} S_{v} k_{r}+G_{f} S_{f} Z_{p s}-C^{\prime} Y_{v}^{*} \delta_{Z}\right) \\
C^{\prime} & =C_{f b}+C_{f f} \\
Y_{v}^{*} & =Y_{v} k_{r} \\
\delta_{Z} & =S_{a} \hat{Z}_{p s}-S_{f} Z_{p s} \\
H_{d} & =H_{\mathrm{ZOH}} H_{i} k_{r} .
\end{aligned}
$$

The disturbance force influence is given by

$$
S_{p}=\frac{v}{F_{\text {dist }}}=\frac{Y_{r}}{Y_{r}\left(Z_{p s}+D\right)+1} .
$$


In its most elaborate form, the following subsystems are used

$$
\begin{aligned}
Y_{r} & =\frac{1}{m_{r} s+b_{r}} \\
C_{f b} & =k_{p}+\frac{k_{i}}{s}+\frac{k_{d} s}{\tau_{d} s+1} \\
C_{f f} & =\mu_{f f} s+\beta_{f f} \\
Z_{p s} & =m_{p s} s, \hat{Z}_{p s}=\frac{\mu_{c} s}{\tau_{c} s+1} \\
H_{\mathrm{ZOH}} & =\frac{1-e^{-s T_{s}}}{s T_{s}}, H_{i}=k_{T} \frac{e^{-s T_{d}}}{\tau_{i} s+1} \\
Y_{v} & =\frac{k_{a} s^{2}+s}{m_{v} s^{2}+b_{v} s+k_{v}} \\
S_{v} & =\frac{1}{\tau_{v} s+1}, S_{f}=\frac{1}{\tau_{f} s+1}, S_{a}=\frac{1}{\tau_{a} s+1} .
\end{aligned}
$$

Here it is assumed $Y_{r}$ describes a stiff robot system.

\section{DERIVATIONS OF STABILITY AND PASSIVITY}

Naive feedback only (Sec. 4.5.3)

Combining Eqs. (4.3) to (4.6) yields apparent admittance

$$
Y_{a}=\frac{1}{s} \frac{m_{v} s^{2}+K_{p} s+K_{i}}{a_{2} s^{2}+a_{1} s+a_{0}},
$$

with $a_{2}=\left(m_{r}+m_{p s}\right) m_{v}, a_{1}=\left(K_{p}+b_{r}\right) m_{v}+K_{p} m_{p s}, a_{0}=K_{i}\left(m_{v}+m_{p s}\right), K_{p}=k_{p} k_{r}^{2}$ and $K_{i}=k_{i} k_{r}^{2}$.

Setting $s=j \omega$, we arrive at

$$
Y_{a}(j \omega)=\frac{\left(m_{v} \omega^{2}-K_{i}\right)-j K_{p} \omega}{a_{1} \omega^{2}+j\left(a_{2} \omega^{3}-a_{0} \omega\right)}=\frac{N_{a}(j \omega)}{D_{a}(j \omega)} .
$$


The positive real condition becomes

$$
\begin{aligned}
& \mathcal{R}\left\{N_{a}\right\} \mathcal{R}\left\{D_{a}\right\}+\operatorname{I}\left\{N_{a}\right\} I\left\{D_{a}\right\} \geq 0, \forall \omega \\
& c_{1} \omega^{4}+c_{2} \omega^{2} \geq 0, \forall \omega \\
& c_{1}=\left(K_{p}+b_{r}\right) m_{v}^{2}-K_{p} m_{r} m_{v} \\
& c_{2}=-K_{i} b_{r} m_{v}
\end{aligned}
$$

For passivity we require that $c_{1} \geq 0$ and $c_{2} \geq 0$. Dividing both conditions by $m_{v}$ (under the constraint that $m_{v}>0$ ) gives passivity conditions (4.7) and (4.8).

Naive Accommodation Control (Sec. 4.5.4)

Combining Eqs. (4.3), (4.4) and (4.6) with $Y_{v}=1 / b_{v}$ yields apparent dynamics

$$
Y_{a}=\frac{\left(K_{p}+b_{v}\right) s+K_{i}}{b_{2} s^{2}+\left(\left(K_{p}+b_{r}\right) b_{v}+K_{i} m_{p s}\right) s+b_{v} K_{i}}
$$

with $b_{2}=\left(m_{r}+m_{p s}\right) b_{v}+K_{p} m_{p s}$. Analogous to the method in the previous section, we set $s=j \omega$ and arrive at the positive real condition

$$
\begin{aligned}
& d_{1} \omega^{2}+d_{2} \geq 0, \forall \omega \\
& d_{1}=\left(K_{p}+b_{v}\right)\left(K_{p}+b_{r}\right) b_{v}-K_{i} m_{r} b_{v} \\
& d_{2}=b_{v} K_{i}^{2}
\end{aligned}
$$

For passivity we require that $d_{1} \geq 0$ and $d_{2} \geq 0$. Dividing $d_{1}$ by $b_{v}$ (under the constraint that $b_{v} \geq 0$, which also conforms directly to $d_{2}$ ) we arrive at the passivity conditions mentioned in the main text.

\section{Naive Stiffness Control (Sec. 4.5.4)}

Analogous to the previous section, setting $Y_{v}=\frac{s}{k_{v}}$ yields apparent admittance

$$
Y_{a}=\frac{K_{r} s^{2}+\left(K_{i}+k_{v}\right) s}{K_{p} m_{p s} s^{3}+\gamma_{2} s^{2}+\left(K_{p}+b_{r}\right) k_{v} s+K_{i} k_{v}},
$$


with $\gamma_{2}=\left(m_{r}+m_{p s}\right) k_{v}+K_{i} m_{p s}$. This system is always stable for positive choice of parameters. Setting $s=j \omega$ yields the positive real condition

$$
\begin{aligned}
& e_{1} \omega^{4}+e_{2} \omega^{2} \geq 0, \forall \omega \\
& e_{1}=K_{p} k_{v} m_{r} \\
& e_{2}=\left(K_{p}+b_{r}\right) k_{v}^{2}+K_{i} b_{r} k_{v} .
\end{aligned}
$$

Since both $e_{1} \geq 0$ and $e_{2} \geq 0$ for positive parameters, this system is always passive.

Element combinations (Sec 4.5.5)

Combining different haptic elements into the virtual dynamics results in additions of passivity conditions. Therefore, we will derive the passivity conditions for a mass-spring-damper system

$$
Y_{v}=\frac{s}{m_{v} s^{2}+b_{v} s+k_{v}}
$$

and show how it relates to the passivity conditions found in the preceding sections.

The apparent admittance for this system becomes

$$
Y_{a}=\frac{m_{v} s^{3}+\left(b_{v}+K_{p}\right) s^{2}+\left(K_{i}+k_{v}\right) s}{m_{r} m_{v} s^{4}+\phi_{3} s^{3}+\phi_{2} s^{2}+\phi_{1} s+K_{i} k_{v}},
$$

with $\phi_{3}=b_{r} m_{v}+b_{v} m_{r}+K_{p} m_{v}, \phi_{2}=b_{r} b_{v}+b_{v} K_{p}+K_{i} m_{v}+k_{v} m_{r}$ and $\phi_{1}=$ $b_{v} K_{i}+b_{r} k_{v}+K_{p} k_{v}$. This system is not necessarily stable for positive choice of parameters. The full stability condition (found by generating the Routh array) is given as:

$$
\begin{aligned}
& b_{v}\left(b_{r}+K_{p}\right)\left(\left[b_{r}^{2}+K_{p}^{2}\right] k_{v} m_{v}+\left[b_{r} b_{v}+b_{v} K_{p}+K_{i} m_{v}\right] K_{i} m_{v}+\right. \\
& \left.\left[b_{r} b_{v}+b_{v} K_{p}+k_{v} m_{r}\right] k_{v} m_{r}+2 b_{r} K_{p} k_{v} m_{v}+b_{v}^{2} K_{i} m_{r}\right) \\
& \geq 2\left(b_{r}+K_{p}\right)\left(b_{v} K_{i} k_{v} m_{r} m_{v}\right) .
\end{aligned}
$$

Note that the 1.h.s. and the r.h.s. share a common factor of $b_{v}\left(b_{r}+K_{p}\right)$. This complicated condition is effectively an upper bound on $K_{i}$. 
Analogous to the method in the previous sections, we set $s=j \omega$ and arrive at the positive real condition

$$
\begin{aligned}
\lambda_{1} \omega^{6}+ & \lambda_{2} \omega^{4}+\lambda_{3} \omega^{2} \geq 0, \forall \omega \\
\lambda_{1}= & b_{r} m_{v}^{2}+K_{p} m_{v}^{2}-K_{p} m_{r} m_{v} \\
\lambda_{2}= & b_{r} b_{v}^{2}+b_{v} K_{p}^{2}+b_{v}^{2} K_{p}+b_{r} b_{v} K_{p}-b_{r} K_{i} m_{v}-b_{v} K_{i} m_{r}-2 b_{r} k_{v} m_{v}+ \\
& K_{p} k_{v} m_{r}-2 K_{p} k_{v} m_{v} \\
\lambda_{3}= & b_{v} K_{i}^{2}+b_{r} k_{v}^{2}+K_{p} k_{v}^{2}+b_{r} K_{i} k_{v} .
\end{aligned}
$$

Inspection of $\lambda_{1}$ shows that it is the same as condition (4.7) (or $c_{1}$ in the analysis of the 'Naive feedback only'). This shows that condition (4.7) is invariant to the addition of other haptic elements. Inspection of $\lambda_{3}$ shows that it is an addition of conditions $d_{2}$ and $e_{2}$ from the analysis on Naive Accommodation and Naive Stiffness control. Inspection of $\lambda_{2}$ shows that it is a summation of $d_{1}, e_{1}, c_{1}$ (i.e. condition (4.8)) and an extra term $\delta_{m k}=-2\left(K_{p}+b_{r}\right) k_{v} m_{v}$. This leads to all the combinations discussed in Sec. 4.5.5 and shown in Table 4.2.

\section{Guideline 1: Using feed-forward control}

We set $Y_{v}=1 / m_{v} s$, and change the control force to

$$
F_{c}=k_{r}\left(\frac{k_{p} s+k_{i}}{s}\left(v_{d}-k_{r} v\right)+\left(\mu_{f f} s+\beta_{f f}\right) v_{d}+G_{f} F_{\text {ext }}\right) .
$$

The apparent admittance becomes

$$
Y_{a}=\frac{1}{s} \frac{\left(\mu_{f f} k_{r}^{2}+\left(1+G_{f} k_{r}\right) m_{v}\right) s^{2}+\left(\beta_{f f} k_{r}^{2}+K_{p}\right) s+K_{i}}{f_{2} s^{2}+f_{1} s+f_{0} s},
$$

with $f_{2}=\mu_{f f} k_{r}^{2} m_{p s}+\left(m_{r}+m_{p s}\right) m_{v}, f_{1}=\left(K_{p}+b_{r}\right) m_{v}+\left(\beta_{f f} k_{r}^{2}+K_{p}\right) m_{p s}$ and $f_{0}=\left(m_{v}+m_{p s}\right) K_{i}$. This system is always stable for positive choice of parameters. Setting $s=j \omega$ yields the positive real condition

$$
\begin{aligned}
& g_{1} \omega^{4}+g_{2} \omega^{2} \geq 0, \forall \omega \\
& g_{1}=\left(G_{f} k_{r}+1\right)\left(K_{p}+b_{r}\right) m_{v}^{2}-\left(K_{p}+\beta_{f f} k_{r}^{2}\right) m_{r} m_{v}+\left(K_{p}+b_{r}\right) \mu_{f f} k_{r}^{2} m_{v} \\
& g_{2}=\left(\beta_{f f} k_{r}^{2}-b_{r}\right) K_{i} .
\end{aligned}
$$


For passivity we require that $g_{1} \geq 0$ and $g_{2} \geq 0$. Dividing $g_{1}$ by $m_{v}$ we arrive at the passivity conditions of Eqs. (4.9) and (4.10). Looking at condition $g_{1} \geq 0$, it can be noted that feed-forward control of the mass reduces the robot inertia from the view of the feedback controller.

\section{Guideline 2: Avoid force filtering}

We apply a first-order low-pass filter to the measured force

$$
S_{f}=\frac{1}{\tau_{f} s+1} .
$$

This makes the virtual dynamics effectively

$$
Y_{v}^{\prime}=Y_{v} S_{f}=\frac{1}{m_{v} s} \frac{1}{\tau_{f} s+1}=\frac{1}{\tau_{f} m_{v} s^{2}+m_{v} s} .
$$

We set $k_{i}=0$, since we know that the naive admittance controller has that requirement for being positive real, and adding a low pass filter will make it worse. The apparent admittance becomes

$$
Y_{a}=\frac{1}{s} \frac{m_{v} \tau_{f} s^{2}+m_{v} s+K_{p}}{h_{2} s^{2}+h_{1} s+\left(K_{p}+b_{r}\right) m_{v}+K_{p} m_{p s}}
$$

with $h_{2}=\left(m_{r}+m_{p s}\right) \tau_{f} m_{v}$ and $h_{1}=\left(\left(K_{p}+b_{r}\right) \tau_{f}+m_{r}+m_{p} s\right) m_{v}$. This gives positive real condition

$$
\begin{aligned}
& n_{1} \omega^{4}+n_{2} \omega^{2} \geq 0, \forall \omega \\
& n_{1}=\left(K_{p}+b_{r}\right) m_{v}^{2} \tau_{f}^{2} \\
& n_{2}=\left(K_{p}+b_{r}\right) m_{v}^{2}-K_{p} m_{v}\left(m_{r}+\left(K_{p}+b_{r}\right) \tau_{f}\right) .
\end{aligned}
$$

For passivity we require that $n_{1} \geq 0$ and $n_{2} \geq 0$. Which results in the passivity condition in (4.11).

Passive physical equivalence of filtered dynamics:

Note that first and second order filters change accommodation and stiffness control into admittance control effectively. Consider pure spring virtual dynamics 
$Y_{v}(s)=s / k_{v}$ in series with a second order low-pass filter $B_{2}(s)$ (with cut-off frequency $\omega_{c}=1 / \tau_{c}$ and relative damping $\zeta$ ):

$$
Y_{v}(s) B_{2}(s)=\frac{s}{k_{v}} \frac{1}{\tau_{c}^{2} s^{2}+2 \zeta \tau_{c} s+1}=\frac{s}{m_{v}^{\prime} s^{2}+b_{v}^{\prime} s+k_{v}},
$$

which is a typical mass-spring-damper admittance form with apparent virtual inertia $m_{v}^{\prime}=\tau_{c}^{2} k_{v}$ and virtual damping $b_{v}^{\prime}=2 \zeta \tau_{c} k_{v}$.

Similarly, for accommodation control, a first order low-pass filter on the measured force will turn the dynamics into admittance control. Consider pure damping virtual dynamics $Y_{v}(s)=1 / b_{v}$ in series with a first order low-pass filter $B_{1}(s)$ (time constant $\tau_{c}$ ):

$$
Y_{v}(s) B_{1}(s)=\frac{1}{b_{v}} \frac{1}{\tau_{c} s+1}=\frac{1}{m_{v}^{\prime} s+b_{v}},
$$

which is a typical mass-damper admittance form with apparent virtual inertia $m_{v}^{\prime}=\tau_{c} b_{v}$. The same holds true for virtual dynamics of spring-damper form with a first order low-pass filter in series.

\section{Guideline 3: compensate post-sensor inertia}

We generate an additional force reading to counteract the post-sensor effects

$$
S_{a} \hat{Z}_{p s} s=\frac{\mu_{c} s}{\tau_{c} s+1} v
$$

We set $k_{i}=0$, since it will interfere with passivity, because we are not changing the inner velocity loop (this assumption was validated). The apparent admittance becomes

$$
Y_{a}=\frac{1}{s} \frac{\left(m_{v} \tau_{c}\right) s^{2}+\left(K_{p} \tau_{c}+m_{v}\right) s+K_{p}}{\left(m_{r}+m_{p s}\right) m_{v} \tau_{c} s^{2}+q_{1} s+q_{2}},
$$

with $q_{1}=\left(\left(K_{p}+b_{r}\right) \tau_{c}+m_{r}+m_{p s}\right) m_{v}+K_{p} m_{p s} \tau_{c}$ and $q_{2}=\left(K_{p}+b_{r}\right) m_{v}+K_{p}\left(m_{p s}-\right.$ $\left.\mu_{c}\right)$. For uncoupled stability we require $q_{2} \geq 0$, which puts an upper bound on the maximum value for $\mu_{c}$. 
The positive real condition becomes

$$
\begin{aligned}
& r_{1} \omega^{4}+r_{2} \omega^{2} \geq 0, \forall \omega \\
& r_{1}=\left(K_{p} m_{v}-K_{p} m_{r}+b_{r} m_{v}\right) \tau_{c}^{2} m_{v} \\
& r_{2}=\left(K_{p}+b_{r}\right) m_{v}^{2}-K_{p} m_{v}\left(m_{r}+\mu_{c}\right)-K_{p}^{2} \mu_{c} \tau_{c} .
\end{aligned}
$$

For passivity we therefore require that $r_{1} \geq 0$ and $r_{2} \geq 0$. Condition $r_{1}$ equals the condition in (4.7). Condition $r_{2}$ is an additional constraint. The total condition is

$$
m_{v} \geq \frac{K_{p}}{K_{p}+b_{r}}\left(m_{r}+\mu_{c}\right)+\frac{K_{p}^{2}}{K_{p}+b_{r}} \frac{\mu_{c}}{m_{v}} \tau_{c}
$$

If $\tau_{c} \rightarrow 0$, the last part on the right hand side of the equation drops and we end up with the condition discussed in the text.

\section{Guideline 4: use some virtual damping}

The virtual dynamics has an additional damping parameter $b_{v}$

$$
Y_{v}=\frac{1}{m_{v} s+b_{v}}
$$

The apparent admittance becomes

$$
Y_{a}=\frac{m_{v} s^{2}+\left(K_{p}+b_{v}\right) s+K_{i}}{\left(m_{r}+m_{p s}\right) m_{v} s^{3}+t_{2} s^{2}+t_{1} s+K_{i} b_{r}},
$$

with $t_{2}=\left(m_{r}+m_{p s}\right)\left(K_{p}+b_{v}\right)+b_{r} m_{v}$ and $t_{1}=\left(m_{v}+m_{p s}\right) K_{i}+\left(K_{p}+b_{r}\right) b_{v}$.

Uncoupled stability of this system is guaranteed if all parameters are positive, since stability condition $t_{2} t_{1} \geq\left(m_{r}+m_{p s}\right) m_{v} K_{i} b_{r}$ always holds in that case.

If we set $s=j \omega$, the positive real condition is found to be

$$
\begin{aligned}
& u_{1} \omega^{4}+u_{2} \omega_{2}+u_{3} \geq 0, \forall \omega \\
& u_{1}=\left(K_{p} m_{v}-K_{p} m_{r}+b_{r} m_{v}\right) m_{v} \\
& u_{2}=b_{v}\left(K_{p}+b_{v}\right)\left(K_{p}+b_{r}\right)-k_{i}\left(b_{r} m_{v}+b_{v} m_{r}\right) \\
& u_{3}=K_{i} b_{v} .
\end{aligned}
$$


Therefore, for strict passivity we require that $u_{1} \geq 0, u_{2} \geq 0$ and $u_{3} \geq 0$. These conditions are discussed in the text. Discriminant analysis would yield too complicated conditions for passivity.

Guideline 5: modify the velocity reference

Although the virtual dynamics is described by a pure inertia, the effective virtual dynamics has the form

$$
Y_{v}^{\prime}=\frac{k_{a} s+1}{m_{v} s} .
$$

The apparent admittance becomes

$$
Y_{a}=\frac{1}{s} \frac{\left(K_{p} k_{a}+m_{v}\right) s^{2}+\left(K_{p}+K_{i} k_{a}\right) s+K_{i}}{v_{2} s^{2}+v_{1} s+\left(m_{v}+m_{p s}\right) K_{i}},
$$

with $v_{2}=m_{r} m_{v}+m_{p s}\left(m_{v}+K_{p} k_{a}\right)$ and $v_{1}=\left(K_{p}+b_{r}\right) m_{v}+\left(K_{p}+K_{i} k_{a}\right) m_{p s}$.

The positive re condition:

$$
\begin{aligned}
& w_{1} \omega^{4}+w_{2} \omega^{2} \geq 0, \forall \omega \\
& w_{1}=\left(K_{p}+b_{r}\right) m_{v}^{2}+\left(\left(K_{p}^{2}+b_{r} K_{p}-K_{i} m_{r}\right) k_{a}-k_{p} m_{r}\right) m_{v} \\
& w_{2}=\left(k_{a} K_{i}-b_{r}\right) K_{i} m_{v} .
\end{aligned}
$$

Therefore, for passivity we require that $w_{1} \geq 0$ and $w_{2} \geq 0$.

Guideline 6: increase velocity loop bandwidth

Apparent Admittance:

$$
Y_{a}=\frac{1}{s} \frac{m_{v} \tau_{d} s^{3}+x_{2}^{\prime} s^{2}+\left(K_{p}+K_{i} \tau_{d}\right) s+K_{i}}{\left(m_{r}+m_{p s}\right) m_{v} \tau_{d} s^{3}+x_{2} s^{2}+x_{1} s+x_{0}},
$$

with $x_{2}^{\prime}=m_{v}+K_{d}+K_{p} \tau_{d}, x_{2}=\left(m_{r}+m_{p s}\right) m_{v}+\left(m_{v}+m_{p s}\right) K_{d}+\left(\left(K_{p}+b_{r}\right) m_{v}+\right.$ $\left.K_{p} m_{p s}\right) \tau_{d}, x_{1}=\left(K_{p}+b_{r}\right) m_{v}+K_{p} m_{p s}+K_{i} \tau_{d}\left(m_{v}+m_{p s}\right)$ and $x_{0}=\left(m_{v}+m_{p s}\right) K_{i}$.

For stability we demand that (derived from the Routh array):

$$
x_{2} x_{1} \geq\left(m_{r}+m_{p s}\right) m_{v} x_{0}
$$


which always holds for positive parameters.

The positive real condition yields

$$
\begin{aligned}
& y_{1} \omega^{6}+y_{2} \omega^{4}+y_{3} \omega^{2} \geq 0, \forall \omega \\
& y_{1}=\left(K_{d}+\left(K_{p}+b_{r}\right) \tau_{d}\right) \tau_{d} m_{v}^{2}-\left(K_{p} \tau_{d}+K_{d}\right) m_{r} m_{v} \tau_{d} \\
& y_{2}=\left(K_{p}+b_{r}\right) m_{v}^{2}+\left(b_{r}\left(K_{d}-K_{i} \tau_{d}^{2}\right)-K_{p} m_{r}\right) m_{v} \\
& y_{3}=-b_{r} K_{i} m_{v} .
\end{aligned}
$$

Therefore, for passivity we require that all parameters are positive, and that $y_{1} \geq 0, y_{2} \geq 0$ and $y_{3} \geq 0$. Using this system with non-zero positive integral gain cannot result in a passive system. The other two passivity conditions are discussed in the text in Sec. 4.6.6.

SYSTEM WITH INTERNAL COMPLIANCE TRANSFER FUNCTION AND POSITIVE REAL CONDITIONS

The equations of motion for the system in Fig. 4.14 are given by

$$
\begin{aligned}
\left(m_{r} \gamma^{\prime} s+b_{r}\right) v^{\prime} & =F_{c}+k_{r} F_{d}+\left(v-v^{\prime}\right)\left(k_{s} / s+b_{s}\right) \\
m_{r} \gamma s v & =F_{\text {ext }}-m_{p s} v s-\left(v-v^{\prime}\right)\left(k_{s} / s+b_{s}\right) .
\end{aligned}
$$

The equations for $Y_{v}, v_{d}$ stay the same as in Eqs. (4.4) and (4.5). Eq. (4.6) changes slightly to

$$
F_{c}=k_{r} \frac{k_{p} s+k_{i}}{s}\left(v_{d}-k_{r} v^{\prime}\right)
$$

The apparent admittance (felt at the distal mass) is given by

$$
Y_{a}=\frac{v}{F_{\text {ext }}}=\frac{1}{s} \frac{z_{4}^{\prime} s^{4}+z_{3}^{\prime} s^{3}+z_{2}^{\prime} s^{2}+z_{1}^{\prime} s+z_{0}^{\prime}}{z_{4} s^{4}+z_{3} s^{3}+z_{2} s^{2}+z_{1} s+z_{0}},
$$


with numerator coefficients

$$
\begin{aligned}
z_{4}^{\prime} & =m_{r} m_{v}(1-\gamma) \\
z_{3}^{\prime} & =\left(K_{p}+b_{r}+b_{s}\right) m_{v} \\
z_{2}^{\prime} & =\left(K_{i}+k_{s}\right) m_{v}+b_{s} k_{p} k_{r} \\
z_{1}^{\prime} & =b_{s} k_{i} k_{r}+k_{p} k_{r} k_{s} \\
z_{0}^{\prime} & =k_{i} k_{r} k_{s} .
\end{aligned}
$$

Denominator coefficients are given by

$$
\begin{aligned}
& z_{4}=m_{v} m_{r}^{2}\left(\gamma-\gamma^{2}\right)+m_{p s} m_{v} m_{r}(1-\gamma) \\
& z_{3}=\left(\left(K_{p}+b_{r}+b_{s}\right) m_{p s}+b_{s} m_{r}+\left(K_{p}+b_{r}\right) \gamma m_{r}\right) m_{v} \\
& z_{2}=\left(b_{r} b_{s}+\left(K_{i}+k_{s}\right) m_{p s}+k_{s} m_{r}+b_{s} K_{p}+\gamma K_{i} m_{r}\right) m_{v}+b_{s} k_{p} k_{r} m_{p s} \\
& z_{1}=\left(\left(K_{p}+b_{r}\right) k_{s}+b_{s} K_{i}\right) m_{v}+\left(b_{s} k_{i}+k_{p} k_{s}\right) k_{r} m_{p s} \\
& z_{0}=K_{i} k_{s} m_{v}+k_{i} k_{s} m_{p s} k_{r} .
\end{aligned}
$$

Because the denominator polynomial is of fourth order, there are two non-trivial conditions to achieve marginal uncoupled stability

$$
\begin{array}{r}
z_{3} z_{2}-z_{4} z_{1}=\Gamma_{1} \geq 0 \\
\Gamma_{1} z_{1}-z_{3}^{2} z_{0} \geq 0 .
\end{array}
$$

These conditions are not insightful and we assume the controller is stable. Stability depends mostly on integral gain $k_{i}$ and internal stiffness $k_{s}$.

Setting $s=j \omega$ we arrive at the positive real condition

$$
\xi_{1} \omega^{8}+\xi_{2} \omega^{6}+\xi_{3} \omega^{4}+\xi_{4} \omega^{2} \geq 0, \forall \omega
$$


with

$$
\begin{aligned}
& \xi_{1}=\left(\gamma^{2}-2 \gamma+1\right) b_{s} m_{r}^{2} m_{v}^{2} \\
& \xi_{2}=\left(-b_{s} k_{i}-k_{p} k_{s}\right) m_{r}^{2} k_{r} \gamma^{2}+\left(k_{s} k_{p} m_{r}+\left(2 m_{v} k_{r}+m_{r}\right) b_{s} k_{i}-\right. \\
& \left.\quad b_{s} k_{p}\left(K_{p}+b_{r}\right)\right) m_{r} k_{r} \gamma+m_{v} b_{s}\left(\left(K_{p}+b_{r}\right)\left(K_{p}+b_{r}+b_{s}\right)-\right. \\
& \left.2 K_{i} m_{r}\right)-m_{r} b_{s}^{2} k_{p} k_{r} \\
& \xi_{3}=\left(\left(b_{r} k_{s}-b_{s} K_{i}\right) m_{r} k_{i} k_{r} \gamma+\left(b_{s} K_{i}^{2}+\left(K_{p}+b_{r}\right) k_{s}^{2}\right) m_{v}-\right. \\
& \left.\quad\left(b_{r} b_{s}^{2} k_{i}+k_{p} m_{r} k_{s}^{2}\right) k_{r}\right) m_{v} \\
& \xi_{4}=-b_{r} k_{i} k_{r} k_{s}^{2} m_{v} .
\end{aligned}
$$

For passivity we therefore require that $\xi_{n} \geq 0, n=1,2,3$ and 4 . From these conditions it becomes hard to determine what constitutes a passive design. Only from $\xi_{1}$ it is required that $\gamma \geq 0$. Condition $\xi_{4}$ is the same as (4.8), stating that no integral velocity gain can be used to remain passive. 
Consider Fig. 5.2, a robot with mass $m_{r}=1 \mathrm{~kg}$ and drive train damping of $b_{r}=0.1 \mathrm{Ns} / \mathrm{m}$. It is admittance controlled to behave like a virtual mass of $m_{v}=0.5 \mathrm{~kg}$ by a position controller with feedback gain $k_{p}=250 \mathrm{~N} / \mathrm{m}$. The admittance controlled device interacts with a spring-like environment given by impedance $Z_{e}(s)=k_{e} / s$, with environment stiffness $k_{e}=1000 \mathrm{~N} / \mathrm{m}$. See the following subsection for the equations of motion and the transfer function for the admittance controlled system*. The admittance of the admittance controlled system is given by $Y_{a}$. The closed loop behavior of the admittance controlled system interacting with the spring-like environment is given by $v_{r} / F_{h}=Y_{a} /\left(1+Y_{a} Z_{e}\right)$. An external force $F_{h}$ of $1 \mathrm{~N}$ acts for $0.001 \mathrm{~s}$ on the system to perturb the system from the origin. Fig. D.1 shows the robot velocity, ingoing $\left(E_{i n}\right)$ and outgoing $\left(E_{\text {out }}\right)$ energy according to the definitions of Nabeel et al. [146] and the exchanged energy between the admittance controlled device and a human $\left(E_{h r}\right)$. These energies are calculated as follows by their time derivative:

$$
\begin{aligned}
\dot{E}_{\text {in }}(t) & = \begin{cases}F_{h}(t) v_{d}(t), & \text { if } F_{h}(t) v_{d}(t)>0 \\
0, & \text { otherwise }\end{cases} \\
\dot{E}_{\text {out }}(t) & = \begin{cases}F_{c}(t) v_{d}(t), & \text { if } F_{c}(t) v_{d}(t)>0 \\
0, & \text { otherwise }\end{cases} \\
\dot{E}_{h r}(t) & =F_{h}(t) v_{r}(t)
\end{aligned}
$$

Nabeel et al. claim that it is sufficient to enforce $E_{\text {out }}(t)$ to be smaller than, or equal to, $E_{i n}(t)$ for the system to be passive at the interaction port $\mathcal{I}_{h r}$. This example shows in Fig. D.1 a counter-example where this condition almost always holds (i.e. $E_{\text {out }}(t)<E_{\text {in }}(t)$ almost always), but the system is unstable (the veloci-

\footnotetext{
${ }^{*}$ Note that for this example $\alpha=1 \mathrm{~m} \beta=0, \gamma=1, k_{d}=0 \mathrm{Ns} / \mathrm{m}$.
} 

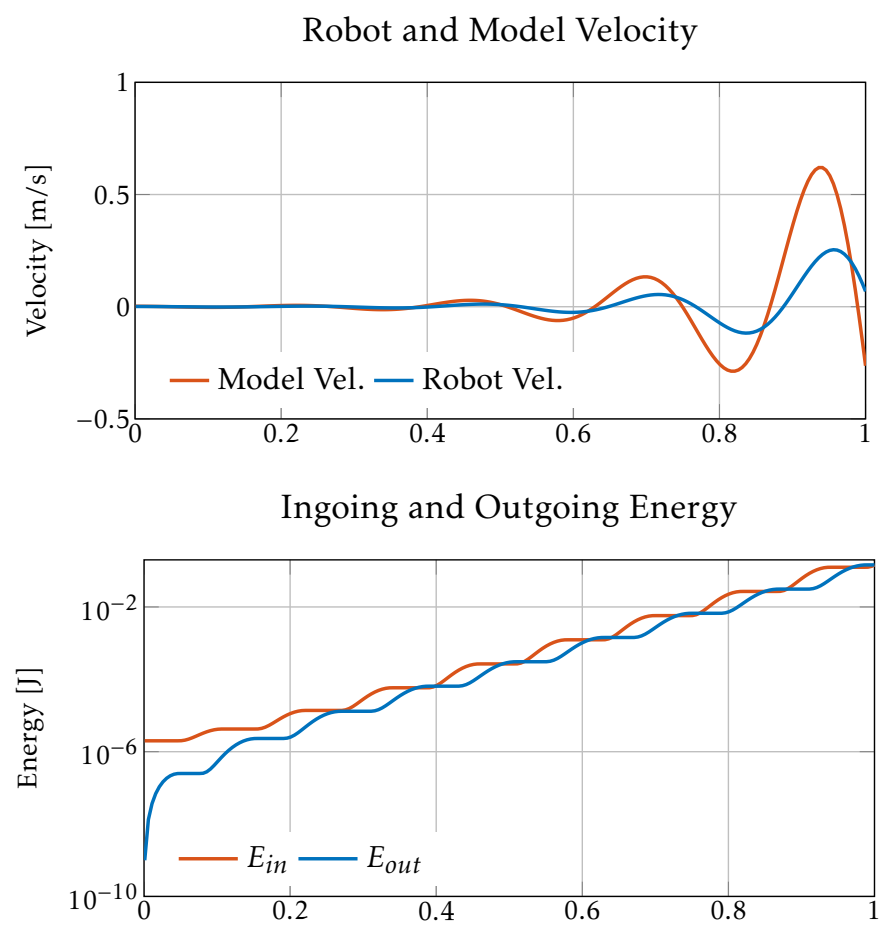

Exchanged Human-Robot Energy

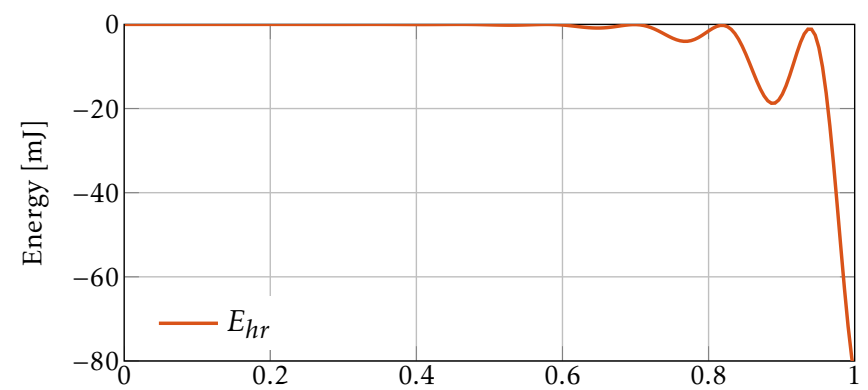

Figure D.1: Top) robot and virtual model velocities. Note that they both show an unstable growing behavior due to the initial system perturbation. Middle) The ingoing and outgoing energies according to Nabeel et al. [146] (see text). Note that $E_{\text {out }}(t)$ is almost always smaller than $E_{i n}(t)$. According to Nabeel et al. this system should be passive at the human-robot interface. Bottom) The energy exchanged at the human-robot interface. Since the energy is negative and becomes more negative over time, it shows that this system is not passive at the human-robot interface. 
ties and positions keep growing) and not passive (i.e. active) at the man-machine interface, because $E_{h r}(t)$ is only negative and becomes more negative over time.

PRC FOR A PARAMETERIZED NAIVE ADMITTANCE CONTROLLER

Consider Fig. 5.5. The equation of motion for the robot system (in the Laplace domain) is given as:

$$
m_{r} s v_{r}+b_{r} v_{r}-\left(k_{d}+\frac{k_{p}}{s}\right)\left(v_{d}-v_{r}\right)=\alpha F_{h}
$$

The equation of motion for the virtual dynamics used in the admittance controller:

$$
m_{v} s v_{d}+\beta\left(k_{d}+\frac{k_{p}}{s}\right)\left(v_{d}-v_{r}\right)=\gamma F_{h} .
$$

This gives a total transfer function for the apparent admittance of the controlled system:

$$
\frac{v_{r}}{F_{h}}=\frac{\alpha m_{v} s^{2}+k_{d}(\alpha \beta+\gamma) s+k_{p}(\alpha \beta+\gamma)}{m_{r} m_{v} s^{3}+\left(\left(b_{r}+k_{d}\right) m_{v}+\beta k_{d} m_{r}\right) s^{2}+\left(k_{p} m_{v}+\beta\left(b_{r} k_{d}+k_{p} m_{r}\right) s+\beta b_{r} k_{p}\right.} .
$$

By setting $s=j \omega$, we arrive at the PRC given in the text (see Chapter 4 and Appendix $\mathrm{C}$ on how to calculate the PRC polynomial)

$$
\begin{aligned}
a_{2} \omega^{4} & +a_{1} \omega^{2}+a_{0} \geq 0, \forall \omega \\
a_{2} & =m_{v}\left(\alpha m_{v}\left(b_{r}+k_{d}\right)-\gamma k_{d} m_{r}\right) \\
a_{1} & =b_{r}\left(k_{d}^{2} \beta(\alpha \beta+\gamma)-k_{p} m_{v}(2 \alpha \beta+\gamma)\right) \\
a_{0} & =\beta b_{r} k_{p}^{2}(\alpha \beta+\gamma) .
\end{aligned}
$$

\section{ENERGY BOOKKEEPING}

The continuous-time controller needs to be time quantized with sample time $T$ following the convention shown in Fig. D.2. The new time index variable used for discrete time is $k$. Due to discretization and non-zero finite calculation times, the control force from the motion controller will be effectively output with one sample time delay (see the Fig. D.2 caption for an explanation). 


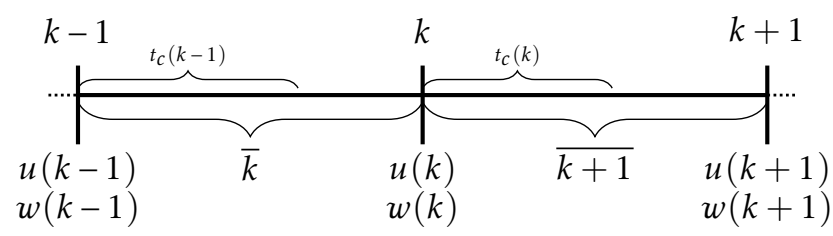

time $\rightarrow$

Figure D.2: Signals $u$ or calculated variables $w$ are sampled and calculated respectively at sample instances $k \in \mathbb{Z}^{+}$. The time leading up to sample instant $k$ is denoted as sample $\bar{k}$. Calculating $w(k)$ actually takes variable non-zero time $t_{c}(k)$. It will take this time duration after the sample instant $k$ for values or signals to be available for output. For proper system determinism, signals calculated during $t_{c}(k)$ are output later at sample instant $k+1$.

The virtual dynamics with semi-implicit Euler integration become

$$
\begin{aligned}
& v_{d}(k+1)=v_{d}(k)-T \frac{\beta(k)}{m_{v}} v_{d}(k)+T \frac{F_{h}(k)}{m_{v}} \\
& x_{d}(k+1)=x_{d}(k)+T v_{d}(k+1) .
\end{aligned}
$$

Here $\beta(k)$ is the variable damper that makes the system SPIS.

For discrete time systems (5.1) can be rewritten as a sum of integrals over all samples (assuming $t$ to be an integer multiple of sample time $T$ )

$$
E(t)=\sum_{k=1}^{t / T}\left(\int_{T(k-1)}^{T k} F(\tau) v(\tau) d \tau\right)=\sum_{k=1}^{t / T} \Delta E(k)
$$

At a power port of impedance causality only, motion is imposed on the device $(v(t))$ while the force applied (e.g. by the actuator) is assumed to be constant during a sampling interval $\bar{F}(\bar{k})$. This gives

$$
\begin{aligned}
\Delta E(k) & =\bar{F}(\bar{k} T) \int_{T(k-1)}^{T k} v(\tau) d \tau \\
& =\bar{F}(\bar{k} T)[x(T k)-x(T(k-1))]
\end{aligned}
$$

which is an exact discrete energy bookkeeping, since the device position $x(t)$ can be exactly retrieved. Energy exchanged with the virtual dynamics behaves in the same way: a constant force on the model generates a state transition with 
position difference $x(k)-x(k-1)$. Therefore, the bookkeeping of virtual energy exchanged from the human to the virtual model is given by

$$
\begin{aligned}
\Delta E_{h v}(k) & =F_{h}(k-1)\left[x_{d}(k)-x_{d}(k-1)\right] \\
E_{h v}(k) & =E_{h v}(k-1)+\Delta E_{h v}(k)
\end{aligned}
$$

The kinetic energy stored in the virtual system is given by

$$
H_{v}(\xi)=\frac{1}{2} m_{v} v_{d}^{2}(k)
$$

Neither $F(t)$ nor $v(t)$ is constant at the $\mathcal{I}_{h r}$ power port of the system. Therefore, the energy exchanged between the human operator and the device cannot be exactly determined. The energy need not be tracked accurately for this method to work. Therefore, the assumption that the input signals from the environment are of a bandwidth lower than the Nyquist frequency of the system is sufficient to get a good approximation of the real exchanged energy between the human and robot $E_{h r}(k)$. 



\section{ACKNOWLEDGMENTS}

The work presented in this dissertation would not have been possible without the help, supervision, moments of fun, moments of frustration, and mental stimulation provided by family, friends and colleagues.

First of all, I would like to thank Arno Stienen for initiating the H-Haptics project's work package at the UT. Your ideas on the subject have guided most of my research. Your supervision has helped me tremendously; especially with generating potential ideas and helping me with scientific writing. I feel that your interest in directly useful applications, and my interest in more fundamental problems and modeling complemented each other well. Assisting you in what is nowadays called the 'Biorobotics minor', which you initiated in 2013, has been an interesting and rewarding experience. Second, I would like to thank my second supervisor and promotor Herman v.d. Kooij. Without you I would literally not have been able to finish my PhD. Your input for my paper on admittance control has been very valuable. Your flexibility during the time I was finishing the work is highly appreciated. Motivated by you and Arno, I have had the experience of teaching quite a few lectures in several courses: Control Engineering for BMT-M9 (2013, now Biorobotics), Biomechatronics, and Human Movement Control. This process helped me appreciate the transfer of knowledge to a new generation of students in such a way that I would like to spend part of my future academic career teaching. Third, I would like to thank the rest of my graduation committee; Stefano Stramigioli, Anton Stoorvogel, André de Boer, Etienne Burdet and David Abbink for taking the time and effort to read and comment on my work.

From my H-Haptics work package I thank Jack Schorsch, my PhD comrade from TU Delft. Collaboration proved to be a bit harder than initially expected, due to diverging research lines. The discussion on robocentric versus anthropocentric lifting of heavy weights has not been fully settled yet, at least not for me! We should definitely revisit that topic in the (near) future. We will, without a doubt, collaborate soon anyway because of your newly developed drive transmission. Freek Tönis from Hankamp Gears and Hankamp Rehab, I thank you for your seemingly infinite patience with the project. I want to thank you for all your time, contributions and ideas. I especially want to mention your flex- 
ibility concerning the myriad of students that have visited Hankamp to work in some way for the project. At the end of the project we have now 'an idea' of what the stronger MAS can do. Let us develop this idea further in the near future. This also leads me to Koen Heuver and Michel van Hirtum, who have been indispensable in designing the automated version of the MAS system. Furthermore, I thank Ruud de Nooij and Linda van den Bedem form Siza and Giuseppe Radaelli and Boudewijn Wisse from Intespring/Laevo for being involved in the project and giving useful user feedback.

Although I thank all other H-Haptics participants and colleagues, I explicitly want to mention David Abbink and Frans v.d. Helm who both oversaw the complete H-Haptics consortium. A special shout-out to Femke van Beek, Jeroen Wildenbeest and Debjyoti Bera for the interesting and stimulating autumn-school week in 2013. It really taught me a lot about human factors research. I also want to mention Irene Kuling, for visiting in 2015 when I was still in Chicago and for an interesting Eurohaptics conference (together with Femke, Erik and Jos) in London in 2016. Piet Lammertse, the admittance control guru from FCS/Moog, I thank for sharing his thoughts and insights on the control method. Jeroen Smeets from the VU I thank for helping us in setting up the Fitts' Law and damping fields research and assisting with obtaining ethical approval for those experiments.

Next, I want to move on to my colleagues at the Biomechanical Engineering group at the University of Twente. I would like to thank Bart Koopman for being my boss. Although we have not discussed much about work, my interaction with you through collaboration with Joan has been very useful, leading to several publications together. Next, I want to thank Martijn Wessels for collaborating in the 4-DOF shoulder exoskeleton research. I still hope that a full size version of your initial design will one day be produced. I want to thank Edsko Hekman for helping me supervise some of my MSc students in mechnical design oriented assignments. I also thank you for useful input about mechanical design in general. Edwin van Asseldonk I thank for teaching me Human Movement Control and explaining about human factors research. Lianne Bode-Bode and Jeanine Lodeweges-De Vries, I thank you both for being the effective group leaders behind the screens. Without your help and your infinite knowledge about the university's bureaucracy, nothing would ever get done!

Ravi Sinha and Gijs van Oort I thank for being my first office mates when I started in 2012. Gijs, I also thank you for your thoughts and insights for the 4-DOF shoulder exoskeleton work we did. Many thanks to Joan Lobo Prat. We 
shared hundreds of rides on the Syntus train between Zutphen and Enschede, discussing many interesting, practical, fundamental and future possibilities of interaction control and intention detection for assistive devices. The trips by train have now become quite dull after you finished your $\mathrm{PhD}$ and moved to the USA. I am also honored you picked me to be one of your paranymphs. I think we all remember how smoothly that went during the ceremony (... not very)! I want to thank Serdar Ates for being a constant source of positivity, even when always working very hard on the Script project's deadlines. We have shared many moments of fun, drank good beer, had good food and exchanged quite a few inappropriate remarks! This, except for the inappropriate remarks, also holds for Israel Mora Moreno. Although you did not spend a lot of time with the group, I thank you for joining Joan, Serdar and me on many occasions. Next, I want to thank Kostas Nizamis. As Joan's 'protégé' you have risen to become an indispensable member of the group. I like how you are always ready and available to help people. I envy your teaching and presentation skills. You are possibly the most easy-going and chill person to be around and I am very happy that you wanted to be my paranymph. This leads me to my second paranymph: Niek Beckers. I like how we are interested in similar questions regarding human movement control, and how we have managed to incorporates some of this in the Biomechatronics and Human Movement Control Courses. You are a great guy. I thank you for helping me a lot giving input to my work and proofreading several of the chapters in my thesis. My long-time office mate Victor Sluiter is the go-to-guy that knows everything. Your critical view on what we do in the group is extremely useful. The two of us helping students extensively with building and programming their robots in the BMT-M9 projects in 2013 and 2014 is a great memory. Stergios Verros, I thank you for being such a nice guy that is always willing to help. I like how you continue Joan and my ideas for interaction control for the trunk. Jos Meuleman, although you have now been gone a while, I thank you for helping me get started with the whole 'admittance control thing', especially together with Erik and Ralph. It was a pleasant surprise, meeting again at the Eurohaptics conference in 2016. I have had some great times with Alex Kuck. Thank you for visiting me in Chicago, where we met some... interesting people. We share a lot of the same ideas and wishes for the world and the scientific community. I hope you will be come back to Enschede, or at least the Netherlands, to collaborate academically or in our own projects. I thank Carsten Voort for helping me with setting up the first setup for the Biomechatronics Haptics practical. Furthermore, I like our continued col- 
laboration into interaction control between our group and your company. I also thank Geert Folkertsma from the Robotics and Mechatronics (RaM) group. We were colleagues at RaM briefly, but we go way back to the first year of the university's Honors Program. It was too bad that, in the limited time left, we were not able to incorporate your energy based control ideas into admittance control that easily. However, collaboration between us and our groups will ensure that this will happen soon!

I also thank the Surgical Robotics guys Guus Vrooijink, Federico Ongaro and Klaas Jelmer Boskma from Groningen for the fun time in Singapore during Biorob 2016. And, of course, I thank the other members of the group that were also there during (part of) my PhD who contributed to making the working day more bearable; (in random, but somewhat chronological, order) Youri van Veen, Jan de Jong, Bas Behrens, Wouter Abbas, Letian Wang, Bram Koopman, Gerdine Meijer, Ard Westerveld, René Fluit, Vincenzo Carbone, Iris Hoogendoorn, Thijs Lohuis, Daphne Boere-van Baal, Antoon Philippi, Bob Giesberts (also for helping with the thesis cover!), Claudia Haarman, Hielke Kiewiet, Floor Kampfens, Denise Engelhart, Tjitske Boonstra, Mark Vlutters, Amber Emmens, Heidi Witteveen, Teun Stortelder, Martijn Grootens, Kyrian Staman, Quint Meinders and everyone else that I forgot to mention!

During my sabbatical at the Physical Therapy and Human Movement Sciences at Northwestern University in Chicago I had the opportunity to work with several amazing people. I would like to explicitly thank Jules Dewald and Ana Maria Acosta for the possibility to spend six months at the lab to work on the NACT3D robot. I appreciate your trust in my work and our continuing collaboration. I thank Netta Gurari for sharing her insights and comments on some of my work. I look back very positively on the more colloquial and practical collaboration with Stuart Traxel and Paul Krueger working on the robot.

Next, I would like to thank all the Bachelor's students, Master's students and interns that I have enjoyed supervising during (or even after, if the work wasn't finished...) my time as a $\mathrm{PhD}$ candidate. I hope you learned as much from me as I learned from you; Maarten Pijper, Ralph Macke (twice), Erik Euving (twice), Floris Bloembergen, Nienke Schimmel, Koen Heuver, Anke Nesselaar, José Alves, Bárbara Cardoso, Dave Vogel, Dennis Niehoff, Nienke Bink, Robert Roos, Stefan van Leuveren, Alexander Roosendaal, Martijn Schuurman, Harm den Dekker, Rik Fierkens and Joeri Landman (twice). Tom Lankhorst, although you have never been my student directly you have been more of a colleague. The DISC summer-school in 2016 was more enjoyable with your and Erik's presence. 
I want to explicitly mention the contributions of the following students; Floris, thank you for visualizing Arno's and my initial idea of the shoulder ROM visualization discussed in Chapter 2. Robert, thank you for getting the 4-DOF shoulder test-setup to work. It was used for results in Chapter 3, and thanks to your help with the setup that chapter will be published in IEEE-TNSRE. I thank, Ralph and Erik for being my first BSc students whom I supervised together with Jos. You helped me with my initial investigations into fundamental issues with admittance control. Our collaboration has lead me to write Chapter 4, which will be published in the IJRR. Joeri and Erik (again) I would like to thank for their preliminary work into possible time-domain passivity enforcement for admittance control that lead me to write Chapter 5. Finally, Rik Fierkens has been a tremendous help in performing experiments, and discussing about optimal control models. Your work contributes to the ideas presented in Chapters 6 and 7. Finally, I want to acknowledge the contributions of Michel van Hirtum again; I am very glad you managed to take over all of the design responsibilities for the project at Hankamp. I thank you for all you have done and I congratulate you on receiving your PDEng degree within our project.

I thank all my non work-related friends for our meet-ups and vacation trips. I would like to explicitly mention Sil Nieuwhof; you also know what it is like to climb this hill called 'getting-your-PhD'. We should get back into the music very, very soon!

My penultimate thanks are to my family. Mijn ouders, Carla and Leo. Ik bedank jullie dat jullie dit voor mij mogelijk hebben gemaakt. Jullie morele steun en interesse in mijn bezigheden zijn voor mij de hoofdredenen geweest om dit werk te doen en het te blijven voortzetten. Mijn zus, Cynthia, bedank ik voor het zijn van mijn grote voorbeeld tijdens mijn formatieve jaren. Mijn nichtje Quinte en neefje Jesse, die je samen met Niek aan deze wereld hebt geschonken, zijn een onuitputtelijke bron van plezier en inspiratie.

Finally, I would like to thank Anne. Anne, samen over de wereld en door het leven reizen is een geweldige evaring. Bedankt voor het achter me staan tijdens betere en slechtere dagen van mijn promotietijdperk. Jij, als maatje, aan mijn zijde gedurende de afgelopen zeven en een half jaar is hetgeen dat dit alles betekenis en waarde geeft. Ik hou van je.

Arvid Keemink Zutphen, September 2017 

Arvid Quintijn Léon Keemink was born December $22^{\text {nd }}, 1987$, in Purmerend (the Netherlands). He got his bilingual high school degree (bilingual Atheneum) and International Baccalaureate English A2 in 2005 at the Marnix College in Ede. He studied Advanced Technology for his Bachelor's degree at the University of Twente, with a minor in Aeronautical Engineering. He followed the honors program at the University of Twente from 2007-2009 and was one of the co-founders and secretary of the honors program student association H.V. Ockham.

During is Master's degree in Mechatronics at the University of Twente, he spent four months at the ETH in Zürich (Switzerland) in

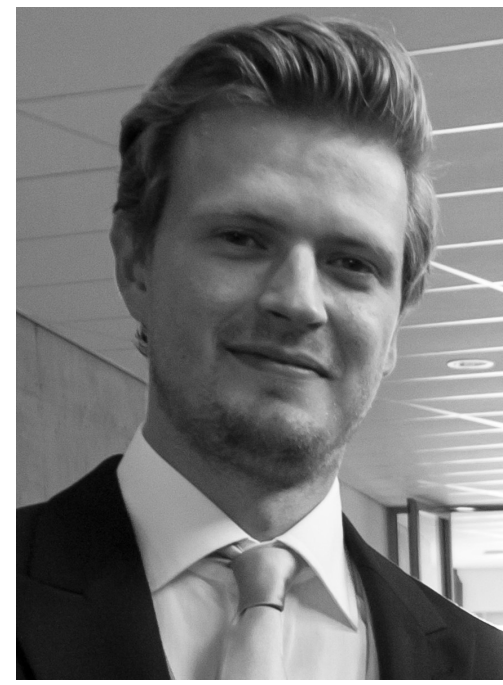
2010/2011 at the Autonomous Systems Laboratory. He received his Master's degree, cum laude, at the Robotics and Mechatronics group at the University of Twente. He won the prize for best MSc thesis at the faculty of EEMCS (Electrical Engineering, Mathematics and Computer Science) for the academic year 2011/2012.

At the beginning of 2012 he started his $\mathrm{PhD}$ in Biomechatronics at the Biomechanical Engineering department at the University of Twente, under supervision of prof. dr. ir. Herman van der Kooij and dr. ir. Arno H.A. Stienen. He was also involved in teaching control engineering and supervised seven BSc students, seven MSc students and five interns during the course of his PhD.

During his $\mathrm{PhD}$ he spent six months abroad at the Physical Therapy and $\mathrm{Hu}-$ man Movement Sciences department at Northwestern University in Chicago (Illinois, USA) to implement admittance control on a custom robot to evaluate human motor control after stroke.

$\mathrm{He}$ is currently investigating human-in-the-loop and self-learning optimizing controllers based on models of neuromuscular reflexes in humans to enable spinal-cord-injury patients to walk with lower-extremity exoskeletons. 

JOURNAL PUBLICATIONS:

7. A.Q.L. Keemink, H. v.d. Kooij and A.H.A. Stienen, "Admittance Control for Physical Human Machine Interaction", accepted for publication in the International Journal of Robotics Research.

6. A.Q.L. Keemink, G. van Oort, M. Wessels and A.H. A. Stienen, "Differential Inverse Kinematics of a Redundant 4R Exoskeleton Shoulder Joint", accepted for publication in IEEE Transactions on Neural Systems and Rehabilitation Engineering.

5. J. Lobo-Prat, K. Nizamis, M.M. Janssen, A.Q.L. Keemink, P.H. Veltink, H.F.J.M. Koopman and A.H.A. Stienen "Comparison between sEMG and force as control interfaces to support planar arm movements in adults with Duchenne: a feasibility study", Journal of NeuroEngineering and Rehabilitation, vol. 14, no. 1, p. 73, 2017.

4. J.F. Schorsch, A. Q. L. Keemink, A.H.A. Stienen, F.C.T. van der Helm, and D.A. Abbink, The Influence of Human-Robot Interaction Order During Fast Lifting Tasks for Different Levels of Weight Compensation, IEEE Transactions on Human Machine Systems, accepted for publication October 2016.

3. J. Lobo-Prat, A.Q.L. Keemink, A.H.A. Stienen, A.C. Schouten, P.H. Veltink, and H.F.J.M. Koopman, "Evaluation of EMG, force and joystick as control interfaces for active arm supports", Journal of NeuroEngineering and Rehabilitation, vol. 11, no. 1, p. 68, 2014.

2. J.F. Schorsch, A.Q.L. Keemink, A.H.A. Stienen, F.C.T. van der Helm and D.A. Abbink, "A novel self-aligning mechanism to decouple force and torques for a planar exoskeleton joint", Mechanical Sciences, vol. 2, no. 5, 2014.

1. M. Fumagalli, R. Naldi, A. Macchelli, F. Forte, A.Q.L. Keemink, S. Stramigioli, R. Carloni, and L. Marconi, "Developing an Aerial Manipulator Pro- 
totype: Physical Interaction with the Environment", IEEE Robotics \& Automation Magazine, vol. 21 , no. 3, p. 41, 2014

INTERNATIONAL PEER-REVIEWED CONFERENCE PUBLICATIONS:

11. R.J.L. Macke, A.Q.L. Keemink and A.H.A. Stienen, "Passive Lower Back Moment Support in a Wearable Lifting Aid: Counterweight Versus Springs", Wearable Robotics: Challenges and Trends, 2016.

10. H.A. den Dekker, N.W.M. Beckers, A.Q.L. Keemink, H. van der Kooij and A.H.A. Stienen, "Assessing the Usability of Remote Control Servos for Admittance-Controlled Haptic Finger Manipulators”, IEEE/RAS-EMBS International Conference on Biomedical Robotics and Biomechatronics (BioRob), 2016.

9. A.Q.L. Keemink ${ }^{\dagger}$, R.I.K. Fierkens ${ }^{\dagger}$, J. Lobo-Prat, J.S.F. Schorsch, D.A. Abbink, J.B.J. Smeets and A.H.A. Stienen, "Using Position Dependent Damping Forces around Reaching Targets for Transporting Heavy Objects: A Fitts' Law Approach", IEEE/RAS-EMBS International Conference on Biomedical Robotics and Biomechatronics (BioRob), 2016.

8. P.N. Kooren, J. Lobo-Prat, A.Q.L. Keemink, M.M. Janssen, M.I. Paalman, A.H.A. Stienen and H.F.J.M. Koopman, "Design and Control of the Active A-Gear: a Wearable 5 DOF Arm Exoskeleton for Adults with Duchenne Muscular Dystrophy", IEEE/RAS-EMBS International Conference on Biomedical Robotics and Biomechatronics (BioRob), 2016.

7. A.H.A. Stienen ${ }^{\dagger}$ and A.Q.L. Keemink ${ }^{\dagger}$, "Visualization of Shoulder Range of Motion for Clinical Diagnostics and Device Development", IEEE/RASEMBS International Conference on Rehabilitation Robotics (ICORR), 2015.

6. J. Lobo-Prat, A.Q.L. Keemink, H.F.J.M. Koopman, A.H.A. Stienen and P.H. Veltink, "Adaptive Gravity and Joint Stiffness Compensation Methods for Force-Controlled Arm Supports", IEEE/RAS-EMBS International Conference on Rehabilitation Robotics (ICORR), 2015.

5. K. Nizamis, J. Lobo-Prat, A.Q.L. Keemink, R. Carloni, A.H.A. Stienen, and H.F.J.M. Koopman, "Switching proportional EMG control of a 3D endpoint arm support for people with duchenne muscular dystrophy", IEEE/

\footnotetext{
${ }^{\dagger}$ Indicates equal contribution.
} 
RAS-EMBS International Conference on Rehabilitation Robotics (ICORR), 2015.

4. J. Lobo-Prat, P.N. Kooren, A.Q.L. Keemink, M.I. Paalman, E.E.G. Hekman, P.H. Veltink, A.H.A. Stienen, and H.F.J.M. Koopman, "Design and control of an experimental active elbow support for adult Duchenne Muscular Dystrophy patients", IEEE/RAS-EMBS International Conference on Biomedical Robotics and Biomechatronics (BioRob), 2014.

3. J.F. Schorsch, A.Q.L. Keemink, A.H.A. Stienen, F.C.T. van der Helm and D.A. Abbink, "The influence of human-robot interaction order during fast lifting tasks for different levels of weight compensation", IEEE/RAS-EMBS International Conference on Biomedical Robotics and Biomechatronics (BioRob), 2014.

2. J.F. Schorsch, A.Q.L. Keemink, A.H.A. Stienen and D.A. Abbink, "A Novel Self-Aligning Mechanism to Decouple Forces and Torques for a Planar Exoskeleton Joint”, Design of Medical Devices Conference: Europe, 2013.

1. A.Q.L Keemink, M. Fumagalli, S. Stramigioli and R. Carloni, "Mechanical design of a manipulation system for unmanned aerial vehicles", IEEE International Conference on Robotics and Automation (ICRA), 2012. 


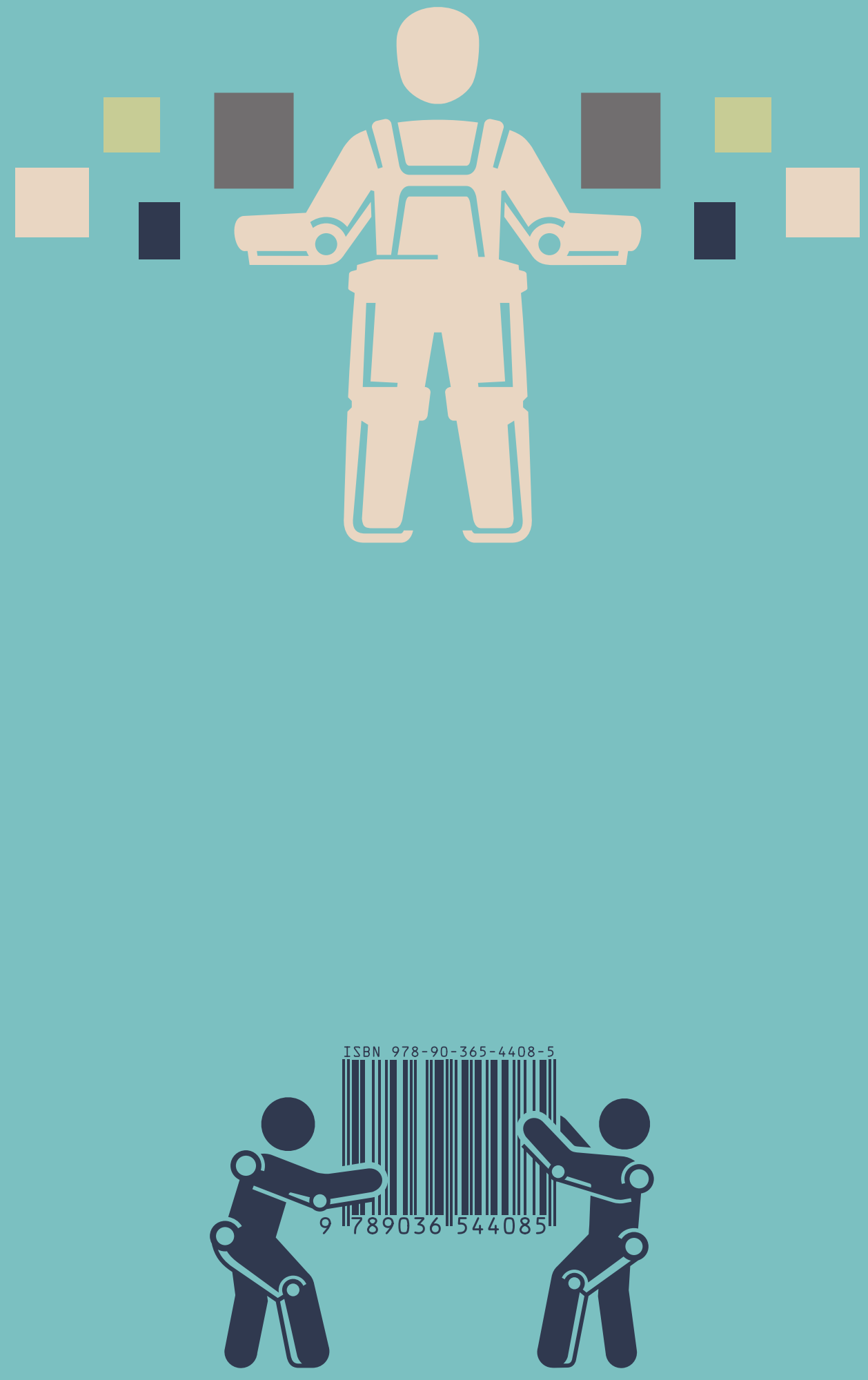